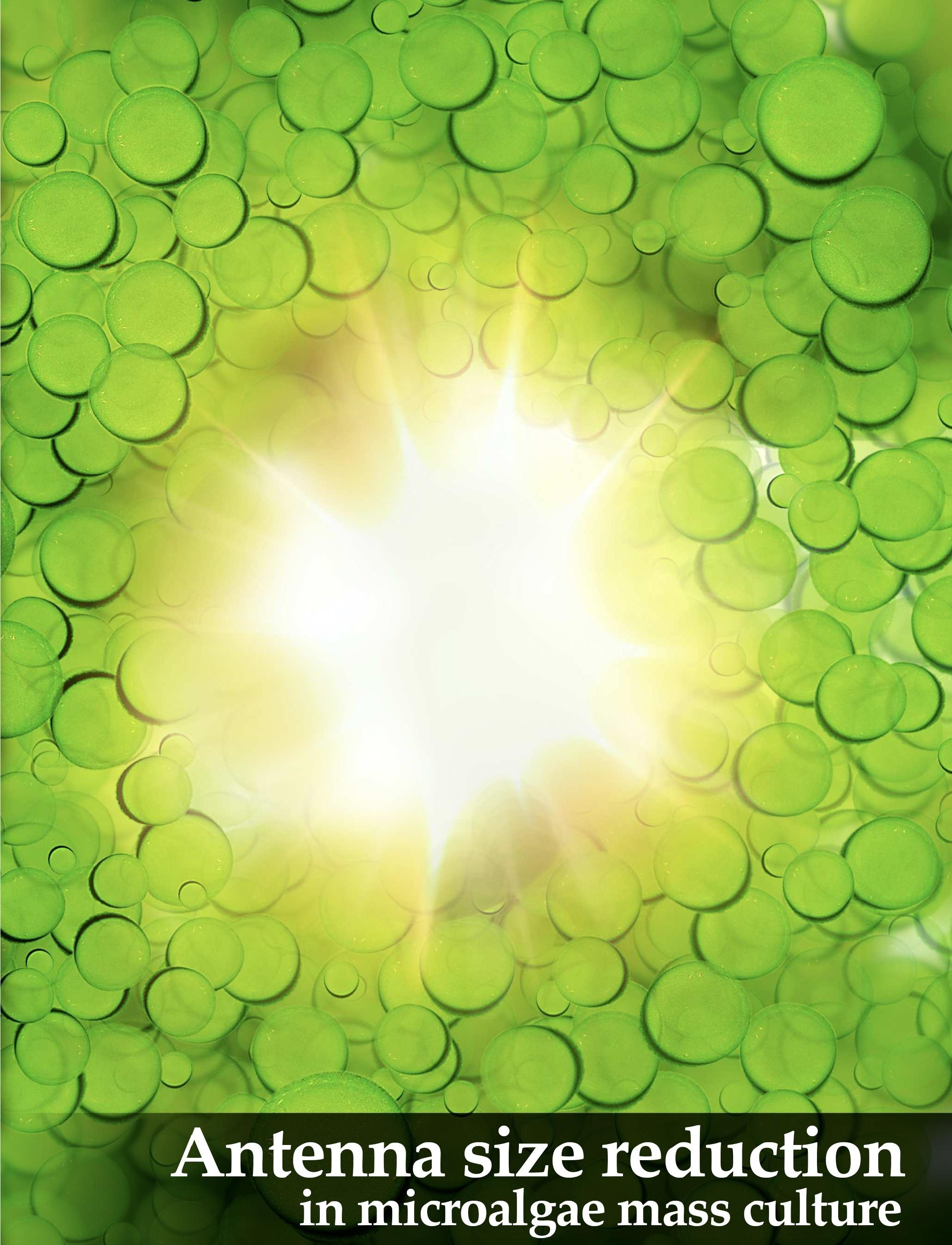

Tim de Mooij 


\section{Antenna size reduction in microalgae mass culture}

Tim de Mooij 


\section{Thesis committee}

\section{Promotor}

Prof. Dr R.H. Wijffels

Professor of Bioprocess Engineering

Wageningen University

\section{Co-promotor}

Dr M.G.J. Janssen

Assistant professor, Bioprocess Engineering

Wageningen University

\section{Other members}

Dr T. Morosinotto, University of Padova, Italy

Prof. Dr J. Huisman, University of Amsterdam

Prof. Dr C. Remacle, $\quad$ University of Liège, Belgium

Prof. Dr L.F.M. Marcelis, ～Wageningen University

This research was conducted under the auspices of the Graduate School VLAG (Advanced studies in Food technology, Agrobiotechnology, Nutrition and Health Sciences) 


\section{Antenna size reduction in microalgae mass culture}

Tim de Mooij

Thesis

submitted in fulfilment of the requirement for the degree of doctor at Wageningen University

by the authority of the Rector Magnificus

Prof. Dr A.P.J. Mol,

in the presence of the

Thesis Committee appointed by the Academic Board to be defended in public

on Friday 9 September 2016

at 4 p.m. in the Aula. 
T. de Mooij

Antenna size reduction in microalgae mass culture, 196 pages.

PhD thesis, Wageningen University, Wageningen, NL (2016)

With references, with summary in English

DOI $10.18174 / 387545$ 


\section{Contents}

Chapter 1

1

Introduction and thesis outline

\section{Chapter 2}

Antenna size reduction as a strategy to increase biomass productivity:

9 a great potential not yet realized

\section{Chapter 3}

Impact of light color on photobioreactor productivity

Effect of photoacclimation on microalgae mass culture productivity

Competition between antenna size mutant and wild type microalgae in mass culture

\section{Chapter 6}

General discussion

Dankwoord 185

About the author 193

List of completed training activities 195 


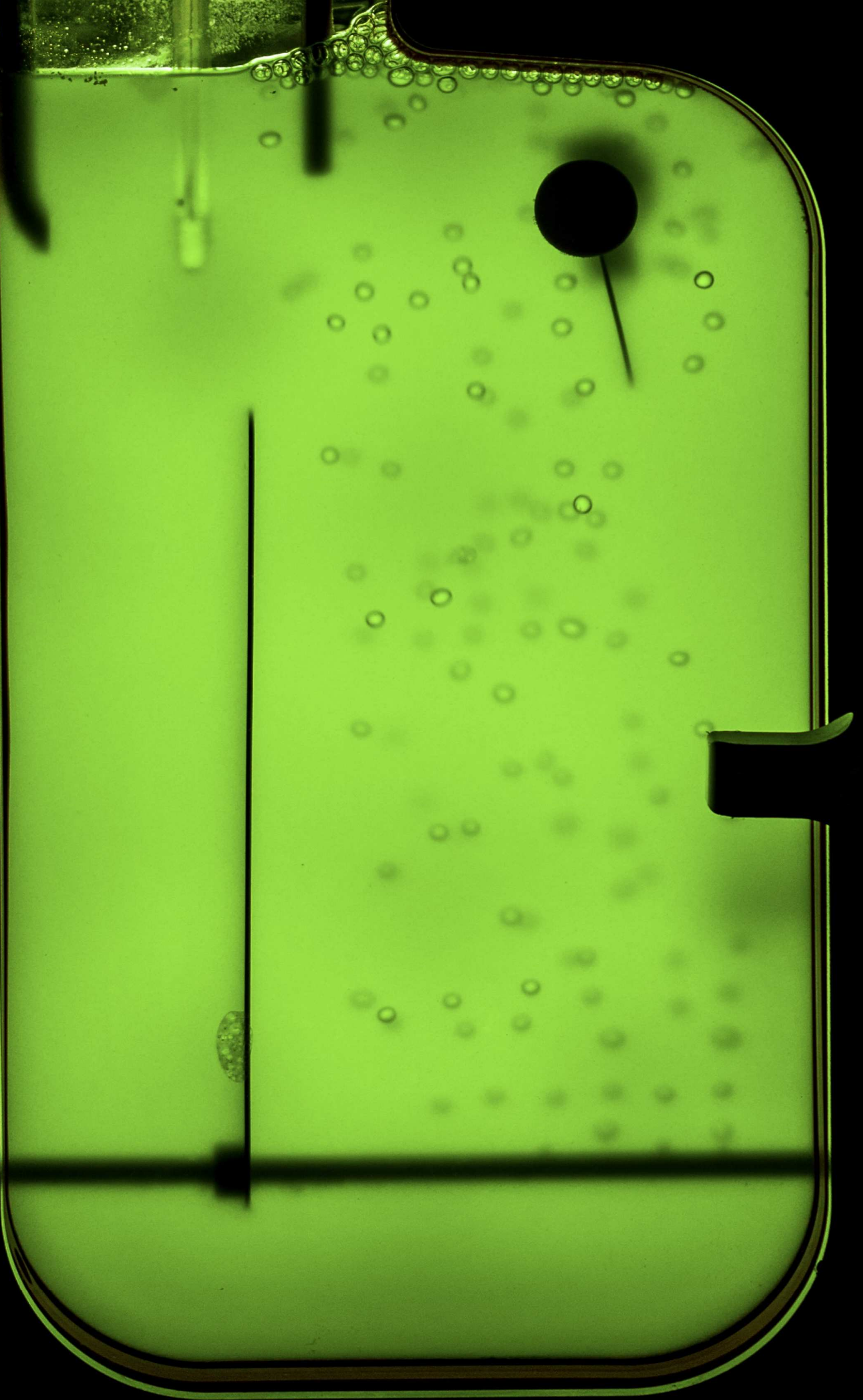





\section{Chapter 1:}

\section{Introduction and thesis outline}




\subsection{Microalgae and photosynthetic efficiency}

Microalgae are unicellular photosynthetic organisms which are usually cultivated in open race-way ponds and in closed systems, photobioreactors. Microalgae have a versatile metabolism, which offers products that can be used as food, feed, fine chemicals, antioxidants, pigments, pharmaceuticals and vitamins (Pulz et al. 2004, de Mooij et al. 2014). Successful commercial exploitation of microalgae for bulk products requires a substantial decrease in the production costs of algal products. A high photosynthetic efficiency is essential to decrease the production costs of microalgal biomass (Norsker et al. 2011). The absorption of light energy is the first step of the photosynthetic process and the amount of light that is absorbed determines the efficiency at which sunlight is converted to biochemical energy. Microalgae can be grown at various light intensities. At low light intensities, microalgae are capable of utilizing light energy at a very high efficiency. At increasing light intensities, the light use efficiency drops as the photosynthetic machinery slowly becomes oversaturated, which leads to wasteful heat dissipation of light energy (Fig. 1.1). The challenge in microalgae cultivation is to maintain a high photosynthetic efficiency at the high light intensities to which algae are exposed under full sunlight.

\subsection{Mass culture conditions}

Commercial large scale cultivation of microalgae requires high biomass concentrations and exposure to full sunlight. The 'mass culture conditions' that are referred to in this thesis are defined as follows: A high incident light intensity that is representative of sunlight conditions in the Netherlands around solar noon $\left(1500 \mu \mathrm{mol}\right.$ photons $\left.\mathrm{m}^{-2} \mathrm{~s}^{-1}\right)$ and a biomass concentration that is high enough to absorb virtually all of this light energy. Because of the steep light gradient in the microalgae culture and the fact that a mass culture is well mixed, the cells experience fluctuating light conditions. The light intensities presented on the X-axis of Fig. 1.1 therefore correspond to different positions inside a microalgae mass culture. In Fig. 1.1A it is shown that the rate of photosynthesis of fully pigmented wild-type microalgae levels off at a certain light intensity. Beyond this photosynthesis saturation point, all additionally absorbed light energy is dissipated as heat which decreases the photosynthetic efficiency. Already at around $10 \%$ of full sunlight, the photosynthetic efficiency of microalgae starts to drop, as is evident from 
Fig. 1.1. Due to this oversaturation bottleneck, in practice microalgae cells near the reactor surface are performing photosynthesis at a low efficiency, while in the deeper parts of the reactor, due to self-shading, little light is available for microalgal growth. Therefore, the primary reason that microalgae cannot be cultivated at a high photosynthetic efficiency under outdoor conditions is the oversaturating intensity of sunlight in combination with its non-homogeneous distribution in photobioreactors. Technical solutions to improve the light intensity distribution in the photobioreactor are at hand, but this principle of light dilution (Tredici et al. 1998, Cuaresma et al. 2011, Dye et al. 2011, Zemke et al. 2013) often leads to high material costs while in the end, the surplus of light absorption should be regarded as a problem of biological origin.
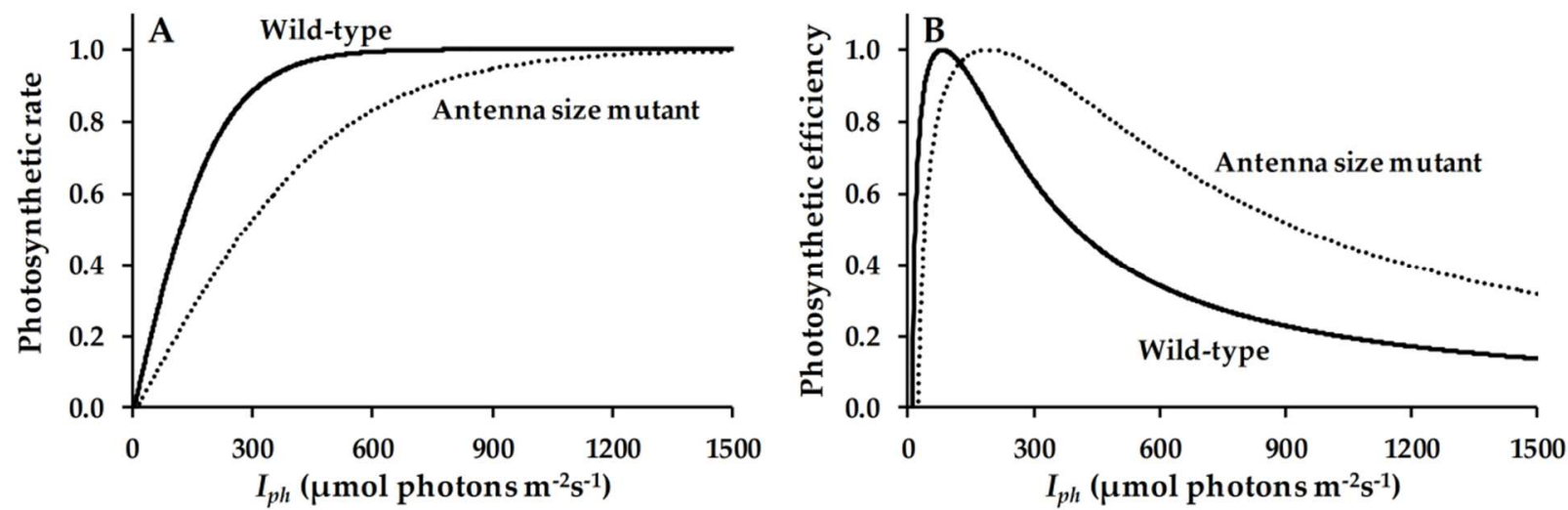

Fig. 1.1 (A) The relative specific rate of photosynthesis as a function of light intensity. (B) The relative photosynthetic efficiency as a function of light intensity. The estimations were made using the model presented in chapter 2.

\subsection{Antenna size mutants}

A biological solution for the oversaturation bottleneck is decreasing the amount of light that is absorbed per cell by reducing the pigment (e.g. chlorophyll) content of the microalgae. This can be accomplished by means of genetic engineering. The pigments make up the antennae complexes, which are the light-harvesting systems that are evident in all known photosynthetic organisms (Grossman et al. 1995). They comprise protein-pigment complexes located in, or on, photosynthetic membranes. With genetic modification, the chlorophyll content of the antennae can be reduced. The obtained 'antenna size mutants' are cells that are more transparent and in theory less prone to oversaturation by sunlight (Fig. 1.2). Because antenna size mutants absorb less light per cell, conversion of solar energy to biochemical energy is expected to occur at a higher 
efficiency (Fig. 1.1). As is clear from Fig. 1.1B, especially at high light conditions, a substantially higher photosynthetic efficiency is to be expected using antenna size reduction.

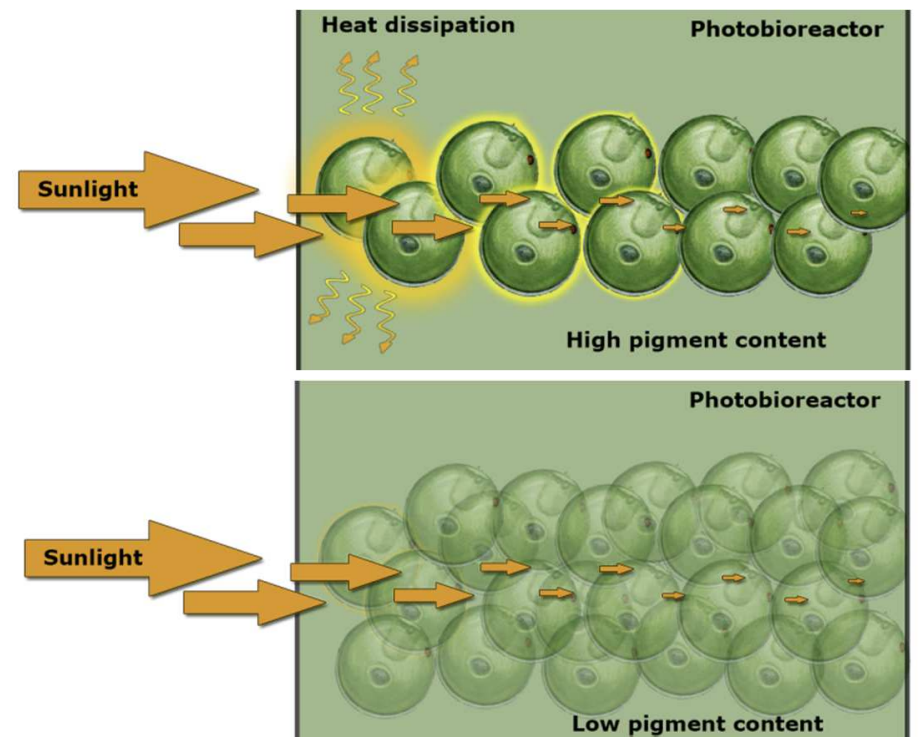

Fig. 1.2 Schematic representation of oversaturation in a photobioreactor. Microalgae cells located close to the light exposed side of the photobioreactor are oversaturated by the high light intensity while shading of other cells. Top: Wild type cells express a high pigment content under mass culture conditions, maximizing their light uptake rate, but leading to oversaturation and heat dissipation. Bottom: Antenna size mutants constitutively express a low pigment content, absorbing less light and therefore they are less sensitive to oversaturation and heat dissipation. Nevertheless, more biomass is required to absorb the same amount of light energy.

\subsection{Thesis outline}

The aim of this thesis is to evaluate the potential of antenna size reduction to increase the biomass productivity in microalgae mass cultures. Insight was obtained in both practical and theoretical limitations of antenna size reduction and how these limitations might be overcome in the next generation of antenna size mutants. In Chapter 2 an experimental setup is presented and recommended to perform biomass productivity measurements under simulated mass culture conditions using antenna size mutants (Fig. 1.3). Antenna size mutant strains of Chlamydomonas reinhardtii and a wild type strain were cultivated in turbidostat controlled lab-scale panel photobioreactors at an incident light intensity of $1500 \mu \mathrm{mol} \mathrm{m}^{-2} \mathrm{~s}^{-1}$. In a large set of experiments, antenna size mutant productivity was compared to that of the wild type strain. Mutant performance was below expectations and it is discussed whether the possible alterations of the light harvesting complexes and the accompanied effect on processes like photoprotection might have played a role in this case.

The rationale behind antenna reduction is the hypothesis that photosystem oversaturation reduces the biomass productivity. Therefore, in Chapter $\mathbf{3}$ the aim was 
to deliver a proof of principle that the light use efficiency of microalgae mass cultures depends on the biomass specific light absorption rate. In this study, artificial LED light of different spectral composition was employed as a tool to generate various biomass specific light absorption rates. This study is based on model estimations which predicted highest productivity for weakly absorbed yellow light in comparison to strongly absorbed red and blue light. These estimations were validated with dedicated laboratory scale experiments under mass culture conditions $\left(1500 \mu \mathrm{mol}\right.$ photons $\mathrm{m}^{-2} \mathrm{~S}^{-}$ 1). Apart from the effect of different light colors on oversaturation, presumed signs of biological interference were observed, and it is discussed whether cultivation of microalgae on monochromatic light could cause a spectral deficiency.

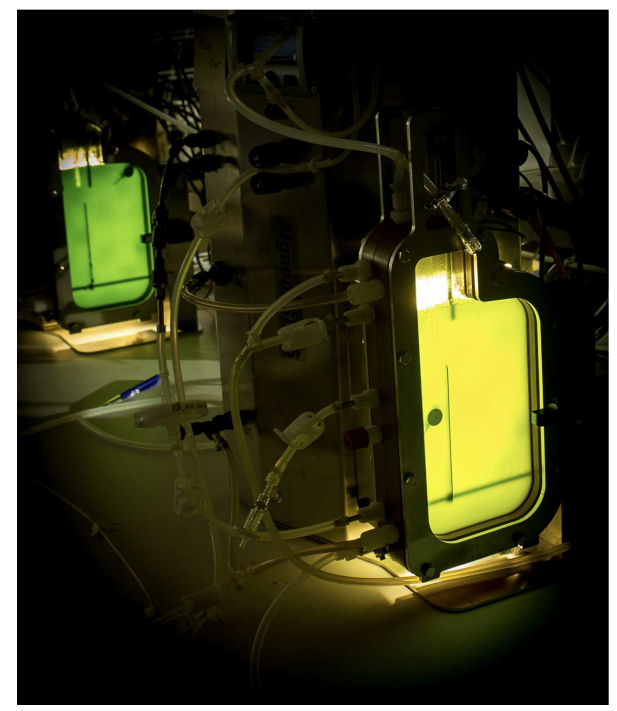

Fig. 1.3 Photograph of laboratory scale photobioreactors containing an antenna size mutant (front) and wild type (back) of Chlamydomonas reinhardtii.

Wild type microalgae also have a natural ability to adjust their pigment content to the light conditions they are exposed to. It was investigated in Chapter 4 whether this process of photoacclimation can be exploited to maximize biomass productivity in wild type mass cultures. Pigment accumulation kinetics were investigated in a light shift experiment. After decreasing the chlorophyll content of Chlorella sorokiniana by exposure to high light conditions, the productivity of low-pigmented cells in a mass culture was measured using two different experimental setups in which a fast recovery of the chlorophyll content was prevented. The findings led to interesting insights in the process of photoacclimation and its effect on microalgae mass cultures. It is also suggested that photoacclimation might be valuable for cultivating microalgae in multi- 
layered systems or biofilms, where local acclimation to the light conditions is advantageous.

In Chapter 5, a modelling approach was chosen to quantify the biomass productivity of mutant mass cultures upon contamination with full antenna species. In simulated competition experiments, the biomass productivity of mutant monocultures was studied under outdoor conditions. The kinetic model is based on experimental data from wild type $C$. sorokiniana and takes into account the spectral dependency of photosynthesis, the effect of day / night cycles, and carbon partitioning between functional biomass and carbohydrate storage. The improvement of biomass productivity resulting from applying antenna size reduction was estimated for the Netherlands and for a location of high irradiance (Tunisia). Furthermore, it was investigated whether the lower competitiveness of antenna size mutants leads to rapid outcompetition after contamination by a full antenna species. The effect of the degree of contamination and antenna reduction on the productivity over the course of an entire summer season are discussed based on the model predictions.

In Chapter 6, an overview of the current understanding of the effect of antenna size reduction on the biomass productivity is provided. A review is given on the potential of antenna reduction technology and a summary is presented of the currently known limitations and knowledge gaps. It is discussed why antenna size reduction is not common in nature and why, analogous to crops, domestication by genetic engineering is essential to overcome limitations regarding light saturation. Recommendations are made on how to increase the competitiveness of antenna size mutants. Finally, an alternative antenna size mutant selection strategy based on selective pressure is proposed that could yield more viable mutants within a shorter timeframe. 
Chapter 1 


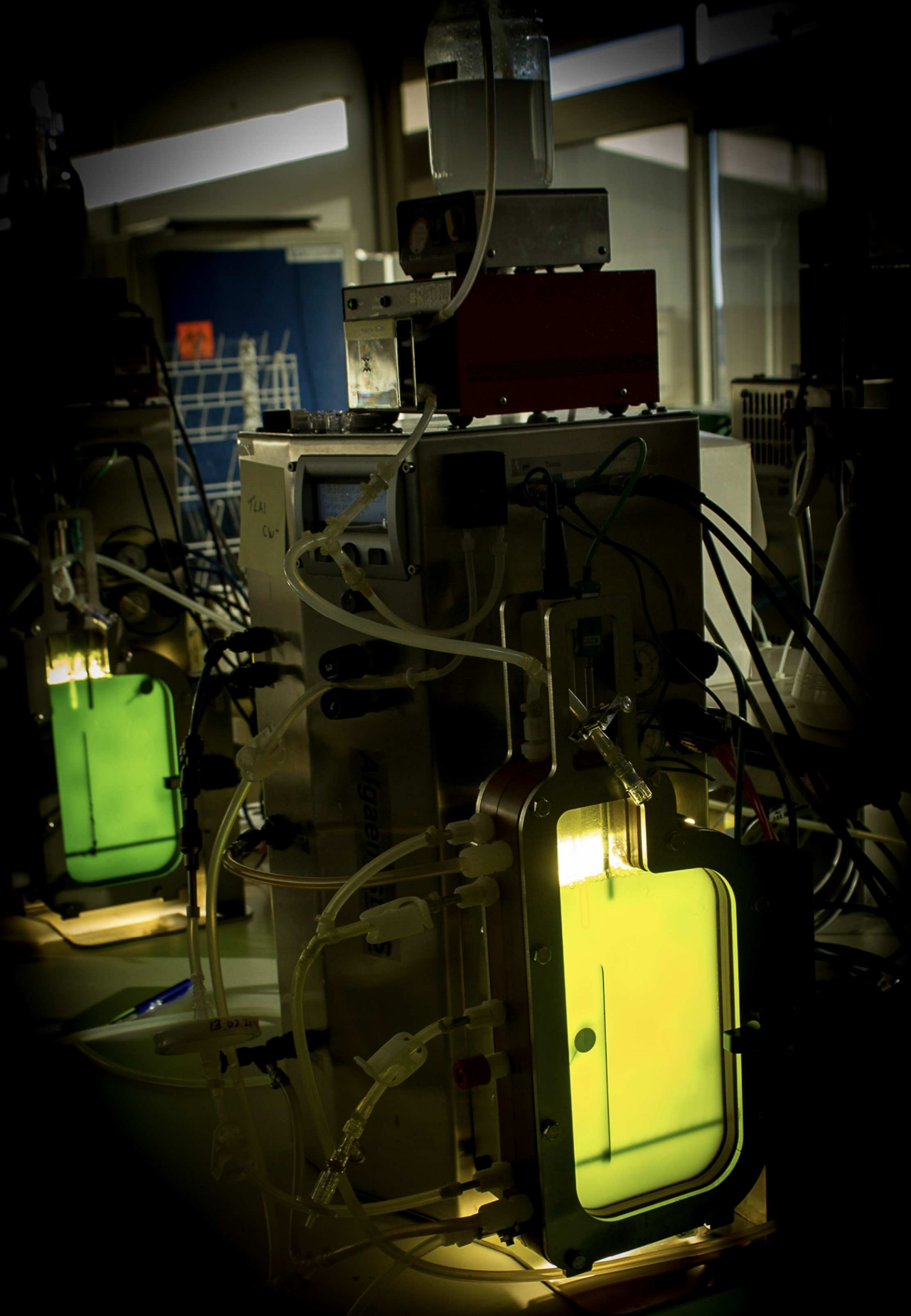




\section{Chapter 2:}

\section{Antenna size reduction as a strategy to increase biomass productivity: a great potential not yet realized}

Tim de Mooij, Marcel Janssen, Jan H. Mussgnug, Olaf Kruse, Matteo Ballottari, Roberto Bassi, Sandrine Bujaldon, Francis-André Wollman, René H. Wijffels 


\begin{abstract}
A major limitation in achieving high photosynthetic efficiency in microalgae mass cultures is the fact that the intensity of direct sunlight greatly exceeds the photosynthetic capacity of the cells. Due to the high pigment content of algal cells, the light absorption rate surpasses the much slower conversion rate to biochemical energy. The excess of light energy is predominantly dissipated as heat, decreasing the light use efficiency of the culture. Algae with a truncated antenna system could substantially increase biomass productivity of mass cultures because oversaturation of the photosystems and concomitant dissipation of light energy, are minimized. In this study, we measured the areal biomass productivity of wild-type strain cultures and four promising antenna size mutant cultures of Chlamydomonas reinhardtii. This was performed under simulated mass culture conditions. The strains were cultivated in turbidostat controlled lab-scale panel photobioreactors at an incident light intensity of $1500 \mu \mathrm{mol} \mathrm{m}^{-2} \mathrm{~s}^{-1}$. The mutant cultures did not exhibit the expected higher productivity. The greatest mutant culture productivity values were approximate to those of the wildtype productivity of $1.9 \mathrm{~g} \mathrm{~m}^{-2} \mathrm{~h}^{-1}$. The high sensitivity to abrupt light shifts indicated that the mutant cultures experienced reduced fitness and higher susceptibility to photodamage. This can plausibly be explained by impaired photo protection mechanisms induced by the antenna complex alterations. Still, if these side effects could be eliminated, the principle of antenna size reduction is a promising strategy to increase productivity. Selection criteria for the future creation of antenna size mutants should, therefore, include tolerance to high light conditions.
\end{abstract}




\subsection{Introduction}

Cultivation of microalgae occurs in open race-way ponds and photobioreactors exploiting sunlight as an energy source. To achieve an economically viable process, high sunlight energy to biomass conversion efficiencies are crucial as they will decrease the production costs of microalgal biomass (Norsker et al. 2011). In relationship to biology, the absorption of light energy is the first step in the photosynthetic process and determines the degree of saturation of the photosynthetic apparatus. At approximately $10 \%$ of full sunlight, photosystems already become saturated which means that the additional absorbed light energy is dissipated as heat or fluorescence. Oversaturation of photosynthesis leads to efficiency losses and photodamage of the photosystems (Melis 1999, Deblois et al. 2013, Vejrazka et al. 2013) and preventing oversaturation would thus increase the biomass yield on light energy.

The primary reason that microalgae cannot be cultivated at a high photosynthetic efficiency under outdoor conditions is the non-homogeneous distribution of sunlight in photobioreactors. Cells near the reactor surface become oversaturated due to being exposed to the intense sunlight, resulting in dissipation of excess light energy. By improving the light energy distribution within the reactor, the effects of oversaturation are alleviated. To date, the concept of light dilution is a frequently employed approach to optimize photobioreactor performance by increasing its surface area per unit of reactor (Tredici et al. 1998, Dye 2010, Cuaresma et al. 2011, Zemke et al. 2013) whereby the light use efficiency can be increased. However, physical and economic limits are being reached in the context of the hardware while there continues to be opportunities for improvement in relationship to biology.

An alternative approach to light dilution is to genetically engineer the microalgal cells to reduce the size of their antenna complexes (Mussgnug et al. 2007, Ort et al. 2011, Formighieri et al. 2012, Perrine et al. 2012, Kwon et al. 2013, Oey et al. 2013). Antennae are light-harvesting systems that are evident in all known photosynthetic organisms (Grossman et al. 1995). They comprise protein-pigment complexes located in, or on, photosynthetic membranes. With genetic modification, the pigment content of the antennae can be reduced. These so-called antenna size mutants are more transparent and are light saturated at greater light intensities than wild-type cells. Antenna size 
mutants absorb less light per cell, however, conversion of solar energy to chemical energy is expected to occur at a higher efficiency as direct sunlight is no longer oversaturating.

From an evolutionary perspective, individual cells benefit from an extensive antenna system because the natural habitat of many microalgae species can be relatively dark. In addition, wasting excess light energy can be advantageous as it is a way to outcompete other photosynthetic organisms, by shading them. Conversely, under mass culture conditions, it is not the individual cell productivity but, instead, the productivity of the culture in its entirety that should be optimized. Therefore, in a controlled mass culture, metabolic costs associated with competition strategies should be minimized. By truncating the light harvesting antennae, cells will not experience oversaturation by light and, consequently, no light energy will be wasted. However, as this provides a competitive disadvantage, maintaining a monoculture of these mutants is of crucial significance.

Studies have demonstrated that, at light intensities that oversaturate wild-type cells, dilute cultures of antenna size mutant have greater photosynthetic activity (i.e., higher oxygen production rate) per unit of chlorophyll (Nakajima et al. 2000, Kirst et al. 2012, Perrine et al. 2012). These findings, however, cannot be translated to the potential of antenna size mutants under mass culture conditions. Since a mass culture possesses a very steep light gradient due to the high biomass density, the light regime in the photobioreactor is completely different. In addition, if measurements are expressed based on chlorophyll without providing the chlorophyll content itself, extrapolation to areal biomass productivity values $\left(\mathrm{g} \mathrm{m}^{-2} \mathrm{~h}^{-1}\right)$ is complicated. As there is no linear relationship between light absorption and chlorophyll content and because chlorophyll is not the only light-harvesting pigment in green algae, expressing the biomass productivity per unit of light absorbance would have been more appropriate and a more valuable benchmark of the efficiency at which light energy is utilized. Another argument in opposition to oxygen evolution experiments is that they originate from short-term measurements (a time scale of minutes), indicating that it cannot be evaluated whether there is a progressive negative effect of the excessive light intensity on the cells in the long term. 
In this study, we present an approach to quantitatively assess the long-term areal biomass productivity of microalgae cultures under simulated mass culture conditions. We employed this approach in order to evaluate the most promising antenna size mutants that are currently available. The performance of the mutants was compared to wild-type reference strains by measurement of the areal biomass productivity in continuous bench-scale culture systems. Under nutrient replete conditions, the areal biomass productivity of an algal culture is determined by the amount of light intensity that it is exposed to and the efficiency at which light energy is converted into biomass. The applied incident light intensity was comparable to direct sunlight, and the biomass concentration was maintained constant utilizing turbidostat control. The applied biomass concentration was sufficient to absorb all incoming light. By combining algal productivity with light absorption rates, the biomass yield on light energy was calculated. This biomass yield on light energy of antenna size mutants and the wild-type strain was employed to evaluate the potential of genetically engineered microalgae with a truncated antenna system in mass cultures.

\subsection{Materials and methods}

\subsubsection{Organisms and medium}

Chlamydomonas reinhardtii CC-1690, CC-124 and TLA2 were obtained from the Chlamydomonas Resource Center (University of Minnesota). More information regarding TLA2 and the other mutant strains of interest (BF4, Stm3LR3, AS2.2) are depicted in Table 2.1 and the indicated publications. All of these antenna size mutants possess partially assembled light harvesting complexes (LHC). They are either deficient in the pigments or the pigment-binding proteins located in the peripheral antenna complexes. The algae were cultivated in a Sueoka high salt (HS) medium with the following composition (in $\mathrm{g} \mathrm{L}^{-1}$ ): urea, 1.48; $\mathrm{KH}_{2} \mathrm{PO}_{4}, 0.706 ; \mathrm{K}_{2} \mathrm{HPO}_{4}, 1.465 ; \mathrm{MgSO}_{4} \cdot 7 \mathrm{H}_{2} \mathrm{O}, 0.560$; $\mathrm{CaCl}_{2} \cdot 2 \mathrm{H}_{2} \mathrm{O}, 0.114$ and $20 \mathrm{~mL} \mathrm{~L}-1$ of a 100 times concentrated Hutner's trace elements solution (Hutner et al. 1950). The medium was filter sterilized (pore size $0.2 \mu \mathrm{m}$ ) and was designed to sustain biomass concentrations up to $9 \mathrm{~g} \mathrm{~L}^{-1}$. The cultures were precultivated in $250 \mathrm{~mL}$ shake flasks containing $100 \mathrm{~mL}$ of medium at $\mathrm{pH} 6.7$, at a light intensity of $200-300 \mu \mathrm{mol} \mathrm{m}{ }^{-2} \mathrm{~s}^{-1}$, and at $25^{\circ} \mathrm{C}$. Cultures were mixed utilizing magnetic stirring bars. 
Table 2.1. Overview of the genetic properties of different antenna size mutants.

\begin{tabular}{|c|c|c|c|c|}
\hline Strain & $\begin{array}{l}\text { Mutation } \\
\text { method }\end{array}$ & Gene involved & Cell wall & References \\
\hline BF4 & UV mutagenesis & unknown & + & $\begin{array}{l}\text { (Olive et al. 1981, De } \\
\text { Vitry et al. 1988) }\end{array}$ \\
\hline Stm3LR3 & $\begin{array}{l}\text { RNA } \\
\text { interference }\end{array}$ & $\begin{array}{l}\text { Genes encoding for } \\
\text { LHCI, LHCII, CP26 and CP29 }\end{array}$ & - & $\begin{array}{l}\text { (Mussgnug et al. } \\
\text { 2007) }\end{array}$ \\
\hline TLA2 & $\begin{array}{l}\text { Insertional } \\
\text { mutagenesis }\end{array}$ & TLA2-CpFTSY & + & (Kirst et al. 2012) \\
\hline AS2.2 & $\begin{array}{l}\text { Insertional } \\
\text { mutagenesis }\end{array}$ & Unknown & + & $\begin{array}{l}\text { (Bonente et al. } \\
\text { 2011) }\end{array}$ \\
\hline
\end{tabular}

\subsubsection{Photobioreactor setup}

The strains were continuously cultivated in flat-panel airlift photobioreactors (Algaemist, Technical Development Studio, Wageningen University, the Netherlands) with a working volume of $0.4 \mathrm{~L}$, an optical depth of $14 \mathrm{~mm}$, and an illuminated area of $0.028 \mathrm{~m}^{2}$ (See Fig. 2.1 for a schematic overview). Light was provided by LED lamps (BXRA W1200, Bridgelux, USA) with a warm-white light spectrum (Fig. 2.2). Reactor temperature was adjusted to $25^{\circ} \mathrm{C}$, and the $\mathrm{pH}$ was maintained at $6.7( \pm 0.1)$ utilizing an on-demand addition of $\mathrm{CO}_{2}$ to a gas stream of di-nitrogen, which was continuously supplied at a rate of $200 \mathrm{~mL} \mathrm{~min}^{-1}( \pm 20)$ in order to mix the suspension and prevent oxygen accumulation. The flow rate was increased to $350 \mathrm{~mL} \mathrm{~min}^{-1}$ to prevent settling of the biomass for the wild-type in the event of floc formation. The photon flux density ( $\mathrm{I}_{\mathrm{ph}}$, $\mu \mathrm{mol} \mathrm{m} \mathrm{m}^{-2} \mathrm{~s}^{-1}$ ) was measured with a LI-COR 190-SA $2 \pi$ PAR $(400-700 \mathrm{~nm})$ quantum sensor (LiCor, USA). The incident light intensity was measured at 28 points evenly distributed over the light exposed surface of the front glass panel of the culture chamber (see Appendix B for light distribution). The light measurement was repeated for every experiment and by averaging the 28 values, the surface-averaged incident light intensity was obtained $I_{p h, i n}$. The outgoing light intensity $I_{p h, o u t}$ was measured in the same manner at the back side of the reactor. $I_{p h \text { out }}$ was measured during the experiment because the transmission of light is dependent on the biomass concentration and pigmentation of the cells. 


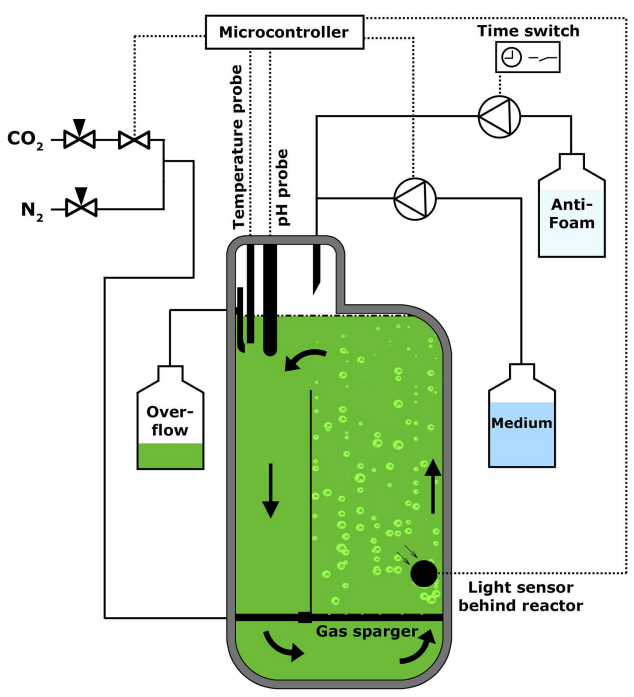

Fig. 2.1 Schematic overview of the experimental setup.

The reactors were operated in turbidostat mode to ensure a constant light regime. The reactors were equipped with a planar photodiode with integrated infrared filter (type SLD-70BG2, Silonex, Canada) behind the rear glass panel to measure the transmission of light through the culture. When light transmission decreased below the selected setpoint, the Algaemist dilution pump (type 400A1, Watson Marlow, UK) was automatically engaged, and the culture was diluted with fresh medium. Each reactor was connected to a harvest vessel via an overflow. Foam formation was prevented by supplying a heat sterilized $2 \%(\mathrm{v} / \mathrm{v})$ antifoam solution (Antifoam B, Mallinckrodt Baker B.V., the Netherlands) which was automatically pumped into the reactor for 1-2 minutes every 2 hours using a time switch. The flow rate (1 to $1.75 \mathrm{ml} \mathrm{min}{ }^{-1}$ ) was adjusted manually depending on the amount of foam that had formed.

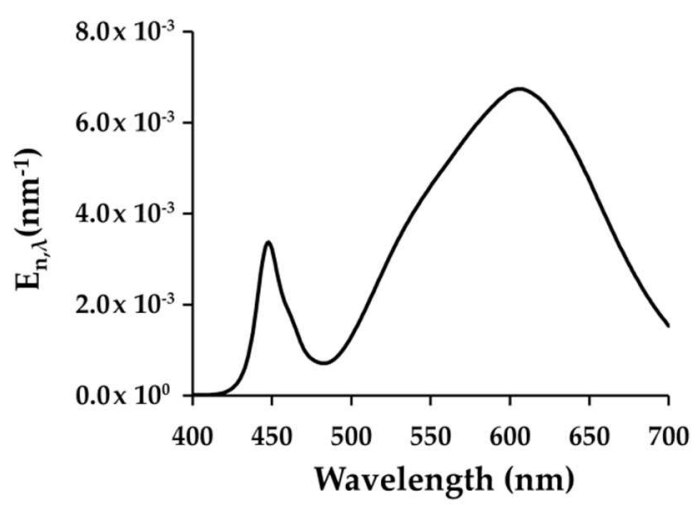

Fig. 2.2 Emission spectrum of the warm-white LEDs (BXRA W1200, Bridgelux, USA). The parameter $E_{n, \lambda}$ represents the relative fraction of PAR photons present within a $1 \mathrm{~nm}$ wavelength interval. 


\subsubsection{Photobioreactor operation}

Reactors were heat-sterilized ( $90 \mathrm{~min}$ at $121^{\circ} \mathrm{C}$ ), and cultures were first grown in batch. The light intensity was increased stepwise as the biomass concentration increased. After the desired biomass concentration was achieved, and turbidostat control was initiated, cultures were allowed to adapt to the cultivation conditions for at least 36 hours. Utilizing turbidostat control, the biomass concentration was maintained at a constant value by automatically diluting the reactor with fresh medium. The biomass concentration and the diluted volume were measured on a daily basis. The average areal biomass productivity was calculated from measurements performed at least 96 hours following the adaptation phase. It was ensured that the cultivation was stable during this period and that the biomass concentration, dilution rate, and areal biomass productivity were constant. Every 24 hours, the culture broth harvested in the overflow vessel was weighed and used for further analyses. The equation to calculate biomass productivity can be derived from the biomass balance and the definition of bioreactor productivity rx (in g m-2h-1):

$\frac{d\left(M_{x, R}\right)}{d t}=\mu \cdot C_{x, R} \cdot V_{R}-F_{\text {out }} \cdot C_{x, \text { out }}$

$r_{x}=\mu \cdot C_{x, R}$

Where $M_{x, R}$ is the biomass dry weight content $(\mathrm{g})$ in the reactor; $C_{X, R}$ is the biomass dry weight concentration in the reactor $\left(\mathrm{g} \mathrm{L}^{-1}\right) ; V_{R}$ is the reactor volume (L); $F_{\text {out }}$ is the harvest flow collected in the overflow vessel $\left(\mathrm{L} \mathrm{h}^{-1}\right)$; and $C_{X, \text { out }}$ is the biomass dry weight concentration of the harvest flow $\left(\mathrm{g} \mathrm{L}^{-1}\right)$. By assuming no biomass accumulation $\left(\mathrm{dM}_{\mathrm{x}, \mathrm{R}} / \mathrm{dt}=0\right)$, equation 3 is obtained.

$0=r_{x} \cdot V_{R}-F_{\text {out }} \cdot C_{x, \text { out }} \Leftrightarrow r_{x}=\frac{F_{\text {out }} \cdot C_{x, \text { out }}}{V_{R}}$

Similarly, it can be deduced from the same biomass balance that the specific growth rate $\mu$ equals the reactor dilution rate $D$ when assuming the reactor volume $V_{r}$ is constant, and the reactor is ideally mixed $\left(C_{X, R}=C_{X, \text { out }}\right)$ :

$\mu=D$ 
Photobioreactor productivity is most effectively evaluated by comparing the areal biomass productivity (in $\mathrm{g} \mathrm{m}^{-2} \mathrm{~h}^{-1}$ ) in which the area reflects the illuminated area of the photobioreactor, and accordingly:

$r_{x}=\frac{F_{\text {out }} \cdot C_{x, \text { out }}}{A_{R}}$

The biomass yield on light energy was calculated using the following equations:

$Y_{x / p h}=\frac{r_{x}}{r_{p h}}$

$r_{p h}=\left(I_{p h, \text { in }}-I_{p h, o u t}\right) \cdot 1 \cdot 10^{-6}$

Where $r_{p h}$ is the areal light absorption rate $\left(\mathrm{mol}_{\mathrm{ph}} \mathrm{m}^{-2} \mathrm{~h}^{-1}\right)$. To calculate $Y_{x / p h}$ on an incident light energy basis, $I_{p h, \text { out }}$ is taken to be zero in eq. 7 .

\subsubsection{Analyses}

\section{Biomass dry weight concentration}

To determine the biomass dry weight content, the culture broth was passed through glass fiber filters as described by Kliphuis et al. (2012) and subsequently, the mass difference between the dried empty filters and the dried filters with algae was recorded. All measurements were performed in triplicate.

\section{Biomass dry weight specific optical cross section}

Light absorption was measured in a double beam spectrophotometer (UV-2600, Shimadzu, Japan) equipped with an integrating sphere (ISR-2600). With this setup, the effect of light scattering on the optical cross section is minimized since forward scatter is collected by the integrating sphere. The light intensity of the two beams following different paths is simultaneously compared. One light path comprised the reference sample (fresh medium) and the other contained the sample of the microalgae suspension. Absorbance was measured from $350 \mathrm{~nm}$ to $750 \mathrm{~nm}$. The average absorbance that was measured between $740 \mathrm{~nm}$ and $750 \mathrm{~nm}$ was subtracted from the absorbance of $350 \mathrm{~nm}$ to $750 \mathrm{~nm}$ which is primarily induced by residual backward and sideward scatter (Dubinsky et al. 1986). Samples were withdrawn directly from the reactor, diluted 1:1 with fresh medium, and transferred to cuvettes (100.099-0S, Hellma, Germany) with a 
short light path of $2 \mathrm{~mm}$. The wavelength-dependent dry weight optical cross section $a_{x, \lambda}$ $\left(\mathrm{m}^{2} \mathrm{~g}^{-1}\right)$ was calculated using equation 8.

$a_{x, \lambda}=\frac{A B S_{\lambda} \cdot \ln 10}{C_{x} \cdot l}$

Where $A B S_{\lambda}$ is the absorbance (-) measured at wavelength $\lambda ; \ln (10)$ is the conversion factor to convert a base 10 logarithm to the natural logarithm; $C_{x}$ is the biomass concentration $\left(\mathrm{g} \mathrm{m}^{-3}\right)$ in the cuvette; and $l$ is the cuvette thickness $(\mathrm{m})$.

\section{Cell number and cell size}

Cell number and cell size were determined with a Beckman Coulter Multisizer 3 (50 $\mu \mathrm{m}$ orifice, Beckman Coulter, Fullerton USA). The samples were diluted with Isoton II dilution buffer. The biomass volume-averaged diameter $\left(\mathrm{d}_{\mathrm{V}, \mathrm{av}}, \mu \mathrm{m}\right)$ of the cells was calculated according to the following equation:

$d_{V, a v}=\sum_{i=0}^{i=N} \frac{V_{p, i}}{V_{p}} \cdot d_{p, i}$

In this equation, $d_{p}$ is the average particle diameter at the interval $[i, i+1] ; V_{p, i}$ is the biomass volume at the interval $[i, i+1]$; and $V p$ is the total biomass volume measured at the interval $[0, N] . N$ is the total number of measurements.

\subsection{Results}

To estimate the potential of antenna size mutants, we constructed a simple mathematical microalgae growth model whereby we calculated the theoretical improvement in photobioreactor productivity by employing antenna size mutants. During long term experiments under simulated mass culture conditions, we measured the areal biomass productivity $\left(r_{x}\right)$ of four different antenna size mutants and two wildtype reference strains. In this context, mass culture conditions are defined as sunlight intensity $\left(1500 \mu \mathrm{mol} \mathrm{m} \mathrm{m}^{-2} \mathrm{~s}^{-1}\right)$ and a biomass concentration $\left(C_{X}\right)$ high enough to absorb all incoming light. For the exact values of light intensity and obtained experimental data of each experiment, please refer to Appendix D.

\subsubsection{Model estimation of biomass productivity and light use efficiency}

Using a simple kinetic model, we predicted the biomass specific growth rate $(\mu)$ and the biomass yield on light energy $\left(Y_{x / p h}\right)$ for both an antenna size mutant and the wild-type 
strain as a function of the light intensity $\left(I_{p h}\right)$. The mutant productivity and yield were estimated using the optical cross section of the BF4 mutant. For a detailed description of the model calculations, please refer to Appendix A.
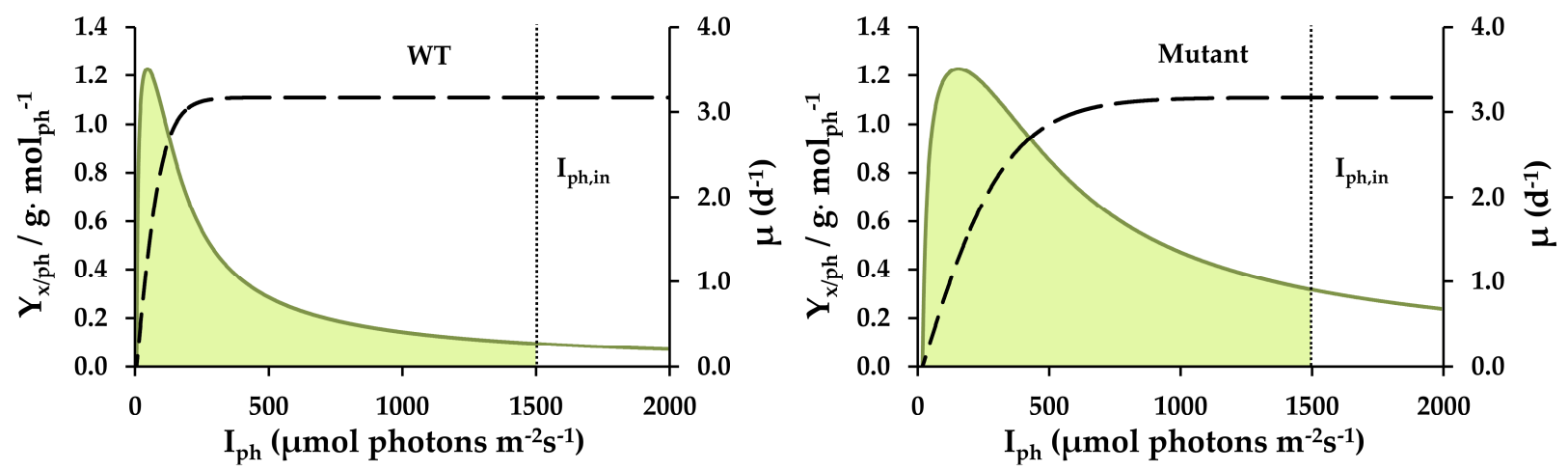

Fig. 2.3 Estimation of the biomass specific growth rate ( $\mu$, dashed line) and biomass yield on light energy ( $Y_{x / p h}$, solid line) of the wild-type strain and an antenna size mutant as a function of the light intensity $\left(I_{p h}\right)$. The mutant performance was estimated using the optical cross section of the BF4 mutant. By numerical integration of the area below the $Y_{x / p h}-I_{p h}$ curves, the biomass productivity per illuminated surface area $\left(r_{x}\right)$ is obtained. The dotted line indicates the incident light intensity used for the experiments in this study.

As exhibited in Fig. 2.3, at non-saturating light intensities, the specific growth rate $\mu$ of an antenna size mutant was predicted to be lower compared to the wild-type because the mutant has less light absorption capacity, limiting the amount of energy available for growth. Antenna size mutants are not expected to have a higher maximum biomass specific growth rate than the wild-type strain. As clearly depicted in Fig. 2.3, the potential of antenna size mutants can be found in their increased light use efficiency at high light intensities compared to the wild-type strain. By absorbing less light, the cellspecific light uptake is reduced to a rate that can continue to be processed efficiently by photosynthesis.

The filled area below the $Y_{x / p h}-I_{p h}$ curve represents the maximal biomass productivity per unit of illuminated surface area $\left(r_{x}, \mathrm{~g} \mathrm{~m}^{-2} \mathrm{~h}^{-1}\right)$. The incident light intensity is 1500 $\mu \mathrm{mol} \mathrm{m} \mathrm{m}^{-2} \mathrm{~s}^{-1}$, and the light intensity at the back side of the photobioreactor is assumed to be equal to the compensation point $I_{p, c}$ of photosynthesis, where net growth is zero. At this point, the $Y_{x / p h}-I_{p h}$ curve crosses the x-axis. The model then predicts an $r_{x}$ of $1.7 \mathrm{~g} \mathrm{~m}^{-}$ ${ }^{2} \mathrm{~h}^{-1}$ for the wild-type strain versus $3.7 \mathrm{~g} \mathrm{~m}^{-2} \mathrm{~h}^{-1}$ for the antenna mutant. Otherwise stated, based on our model estimation, we conjecture a doubling of photobioreactor productivity employing the mutant when compared to the wild-type. 


\subsubsection{Optical cross section of wild-type and antenna size mutants}

The antenna size mutants and the wild type strain were grown in photobioreactors in turbidostat mode as described in the material and methods section. After it was established that $C_{X}$ and $D$ were constant and the cultures were completely acclimated to the applied light regime, the biomass dry weight specific optical cross section $\left(a_{x}\right)$ was measured. The absorption spectra of the wild-type and the mutants, which are normalized to dry biomass weight, were measured to compare the degree of pigmentation (Fig. 2.4). A lower optical cross section corresponds to more transparent cells and thus a more prominent mutant phenotype. It was confirmed that the mutations were successful since all mutants absorbed less light per unit of dry biomass than the wild-type strain. The BF4 mutant demonstrated the lowest pigmentation.

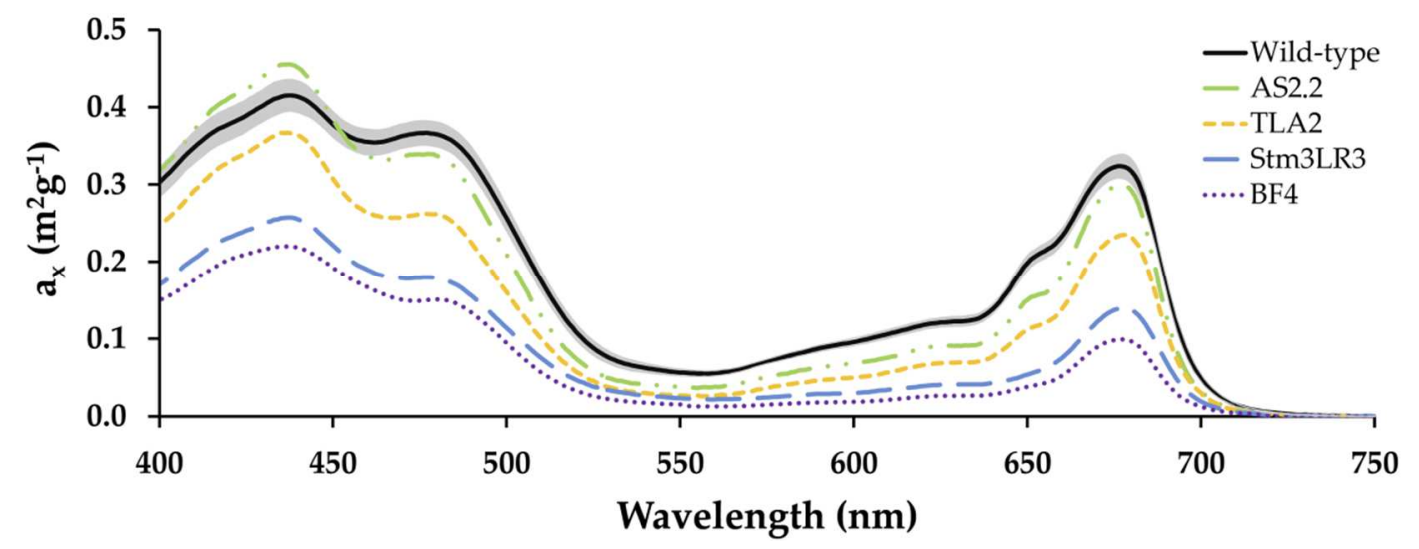

Fig. 2.4 Wavelength dependent biomass dry weight specific optical cross section of four mutant strains and wild-type. Lines represent the average of four reactor experiments $\pm S D$ (wild-type) and a single reactor experiment (mutants). The incident light intensity $\left(I_{p h, i n}\right)$ was $1500 \mu \mathrm{mol} \mathrm{m} \mathrm{m}^{-2} \mathrm{~s}^{-1}$. The culture outgoing light intensity $\left(I_{\text {ph,out }}\right)$ was $10 \mu \mathrm{mol} \mathrm{m}^{-2} \mathrm{~s}^{-1}$ for the wild-type and $50 \mu \mathrm{mol} \mathrm{m} \mathrm{m}^{-2} \mathrm{~s}^{-1}$ for the mutants.

\subsubsection{Areal biomass productivity of wild-type strain}

The areal productivity of the wild-type strains was measured at an incident light intensity $\left(I_{p h, i n}\right)$ of $1500 \mu \mathrm{mol} \mathrm{m} \mathrm{m}^{-2} \mathrm{~s}^{-1}$ in 8 different continuous experiments to provide a robust dataset for comparison with the mutants. The measured areal biomass productivity for the wild-type strain CC1690 was $1.9 \mathrm{~g} \mathrm{~m}^{-2} \mathrm{~h}^{-1}$, the biomass concentration in the photobioreactor was $2.6 \mathrm{~g} \mathrm{~L}^{-1}$ and the biomass specific growth rate was $1.3 \mathrm{~d}^{-1}$. As illustrated in Fig. 2.5, there was variability in the measured productivities. In 4 of the 8 experiments, the areal productivity $r_{x}$ clustered around $1.9 \mathrm{~g} \mathrm{~m}^{-2} \mathrm{~h}^{-1}$ and, in the other 4 
experiments, $r_{x}$ clustered around $2.4 \mathrm{~g} \mathrm{~m}^{-2} \mathrm{~h}^{-1}$. The latter value correlated with the formation of cell agglomerates in 4 of the 8 wild-type experiments, which led to increased productivity values. Since single Chlamydomonas cells featured a size of approximately $8 \mu \mathrm{m}$ during all experiments, cultures expressing agglomerate formation could be clearly discerned. In the other 4 experiments with the wild-type, no agglomeration was detected. The mutant strains at no time exhibited agglomerate formation. To exclude agglomerate formation as a factor influencing productivity, only wild-type experiments without agglomerate formation were compared to the mutant experiments. The averaged results of these 4 experiments are indicated in Fig. 2.6.

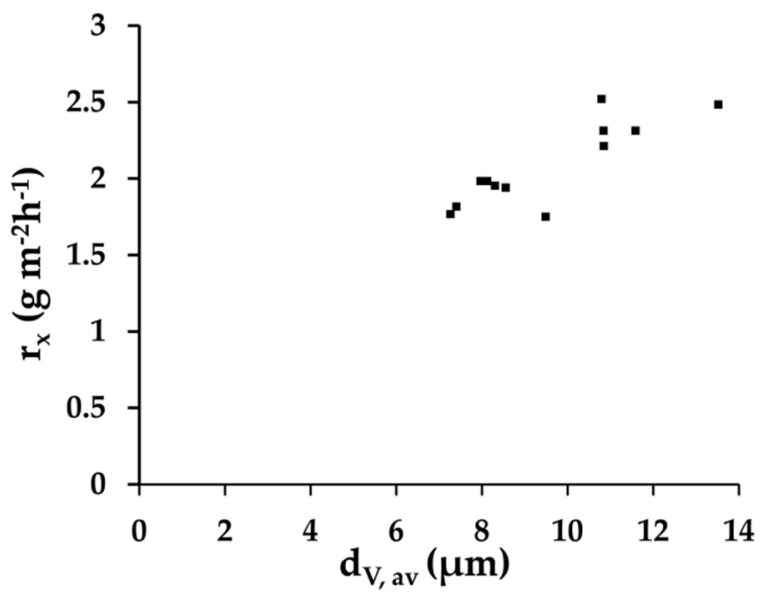

Fig. 2.5 Areal biomass productivity $\left(r_{x}\right)$ of wildtype strain CC1690 as a function of the volume averaged diameter $\left(d_{V, a v}\right)$ of the cells and agglomerates of cells. Each data point originates from a 24 h interval measurement during which $d_{V, a v}$ and $r_{x}$ were determined.

In order to demonstrate the magnitude of oversaturation of the wild-type strain at an $I_{p h, i n}$ of $1500 \mu \mathrm{mol} \mathrm{m}{ }^{-2} \mathrm{~s}^{-1}$, this strain was also cultivated at an $I_{p h, i n}$ of $850 \mu \mathrm{mol} \mathrm{m}^{-2} \mathrm{~s}^{-1}$. At $1500 \mu \mathrm{mol} \mathrm{m}{ }^{-2} \mathrm{~s}^{-1}, r_{x}$ was ascertained to be $1.9 \mathrm{~g} \mathrm{~m}^{-2} \mathrm{~h}^{-1}$ versus $1.5 \mathrm{~g} \mathrm{~m}^{-2} \mathrm{~h}^{-1}$ at $850 \mu \mathrm{mol} \mathrm{m}^{-}$ ${ }^{2} \mathrm{~S}^{-1}$ (Fig. 2.6). $C_{X}$ was found to be substantially higher at $1500 \mu \mathrm{mol} \mathrm{m}{ }^{-2} \mathrm{~s}^{-1}\left(2.6 \mathrm{~g} \mathrm{~L}^{-1}\right)$ than at $850 \mu \mathrm{mol} \mathrm{m} \mathrm{m}^{-2} \mathrm{~s}^{-1}\left(1.9 \mathrm{~g} \mathrm{~L}^{-1}\right)$ because additional biomass is required to absorb the additional light. The dilution rate $(D)$, which is equal to the biomass specific growth rate $(\mu)$, did not increase at an $I_{p h, i n}$ of $1500 \mu \mathrm{mol} \mathrm{m}^{-2} \mathrm{~s}^{-1}$ because the higher light intensity was compensated by a higher biomass concentration. The biomass yield on light energy decreased from $0.51 \mathrm{~g} \mathrm{~mol}_{\mathrm{ph}^{-1}}$ at $850 \mu \mathrm{mol} \mathrm{m} \mathrm{m}^{-2} \mathrm{~s}^{-1}$ to $0.37 \mathrm{~g} \mathrm{~mol}_{\mathrm{ph}^{-1}}$ at $1500 \mu \mathrm{mol} \mathrm{m}^{-2} \mathrm{~s}^{-1}$, demonstrating the effect of photosaturation. 


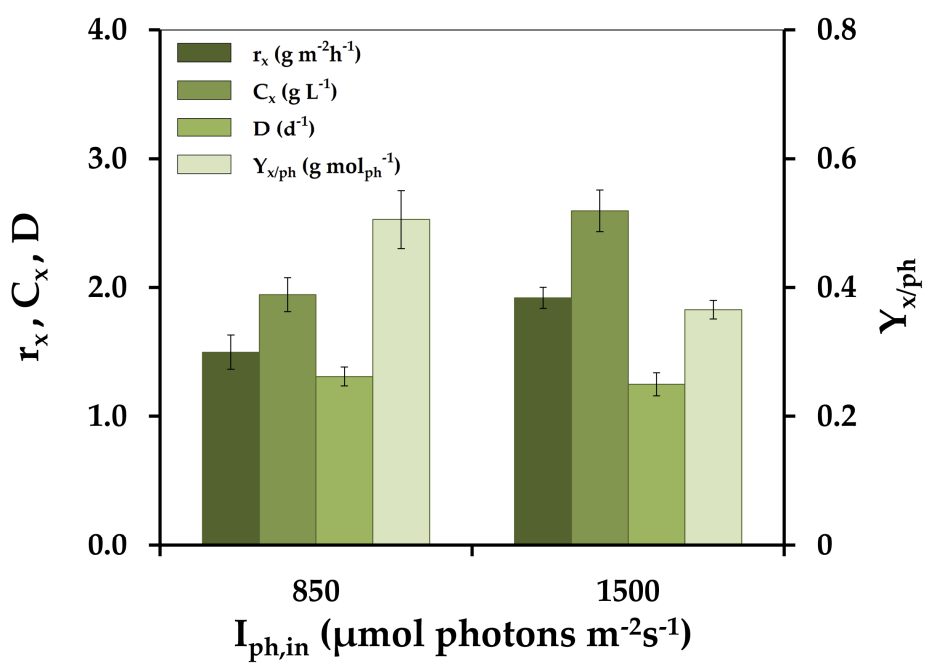

Fig. 2.6 Areal biomass productivity $\left(r_{x}\right)$, dry biomass concentration $\left(C_{x}\right)$, dilution rate $(D)$ and biomass yield on absorbed light $\left(Y_{x / p h}\right)$ of wild-type at medium and high light conditions. Each bar represents the average of $n \geq 4\left(1500 \mu \mathrm{mol} \mathrm{m} \mathrm{m}^{-2} \mathrm{~s}^{-1}\right)$ or $n \geq 5\left(850 \mu \mathrm{mol} \mathrm{m} \mathrm{m}^{-2} \mathrm{~s}^{-1}\right)$ data points \pm pooled SD that were measured on a daily basis within four $\left(1500 \mu \mathrm{mol} \mathrm{m}^{-2} \mathrm{~s}^{-1}\right)$ or two $\left(850 \mu \mathrm{mol} \mathrm{m} \mathrm{m}^{-2} \mathrm{~s}^{-1}\right)$ reactor experiments.

\subsubsection{Areal biomass productivity of antenna size mutants}

The performance of four Chlamydomonas antenna size mutants was compared to that of wild-type strain CC1690 at an $I_{p h, i n}$ of $1500 \mu \mathrm{mol} \mathrm{m}^{-2} \mathrm{~s}^{-1}$. None of the antenna mutants exhibited the anticipated increase in areal biomass productivity. The best performing mutants achieved similar $r_{x}$ values to that of the wild-type (Fig. 2.7 and Fig. 2.8A). We also investigated the effect of the outgoing light intensity on $r_{x}$. For two of the mutants (BF4 and TLA2), $r_{x}$ was significantly higher at an $I_{p h, o u t}$ of $100 \mu \mathrm{mol} \mathrm{m}^{-2} \mathrm{~s}^{-1}$ than at 25 $\mu \mathrm{mol} \mathrm{m} \mathrm{s}^{-2}$.

At an $I_{p h, o u t}$ of $25 \mu \mathrm{mol} \mathrm{m} \mathrm{s}^{-2} \mathrm{~s}^{-1}$, the BF4 mutant, which has the lowest optical cross section $\left(a_{x}\right)$ of all mutants (Fig. 2.4), attained the highest biomass concentration (4.5 $\mathrm{g} \mathrm{L}^{-1}$ ) measured in this series of experiments. In contrast, at an outgoing light intensity of 10 $\mu \mathrm{mol} \mathrm{m} \mathrm{m}^{-2} \mathrm{~s}^{-1}$, wild-type biomass concentration was only $2.6 \mathrm{~g} \mathrm{~L}^{-1}$. Although the BF4 biomass concentration was much higher than that of the wild-type, its specific growth rate $\mu$ was markedly lower, resulting in a lower areal biomass productivity than that of the wild-type.

In all mutant cultures, a higher outgoing light intensity (i.e. a lower biomass concentration) resulted in a higher biomass specific growth rate as more light was available per cell. At an outgoing light intensity of $100 \mu \mathrm{mol} \mathrm{m}^{-2} \mathrm{~s}^{-1}$, AS2.2 and TLA2 
attained even higher specific growth rates than the wild-type. However, this was counterbalanced by a much lower biomass concentration and thus a lower areal productivity than the wild-type.

We attempted to cultivate BF4 and TLA2 at an $I_{p h \text { out }}$ of $10 \mu \mathrm{mol} \mathrm{m}^{-2} \mathrm{~s}^{-1}$, but these cultures collapsed. The cultures were first grown in batch in order to attain the biomass concentration corresponding to $I_{\text {ph,out }}=10 \mu \mathrm{mol} \mathrm{m} \mathrm{m}^{-2} \mathrm{~s}^{-1}$. However, this biomass concentration could not be achieved as growth ceased at higher biomass densities. TLA2 exhibited the same behavior at $I_{p h, o u t}=25 \mu \mathrm{mol} \mathrm{m}{ }^{-2} \mathrm{~s}^{-1}$. To illustrate the effect of pigment reduction on the biomass concentration in a turbidostat controlled photobioreactor with a fixed $I_{p h, i n}$ and $I_{p h, o u t}$, we extrapolated the biomass concentration of the mutants to the same $I_{p h \text {,out }}\left(10 \mu \mathrm{mol} \mathrm{m} \mathrm{m}^{-2} \mathrm{~s}^{-1}\right)$ that was used for the wild-type strain. The extrapolated biomass concentration is depicted as $C_{x}{ }^{\prime}$ in Fig. 2.7 and Fig. 2.8A. Please refer to Appendix $\mathrm{C}$ for the calculation of $C_{X}{ }^{\prime}$. The following extrapolated biomass concentrations were calculated: BF4, $5.5 \mathrm{~g} \mathrm{~L}^{-1}$; TLA2, $3.4 \mathrm{~g} \mathrm{~L}^{-1}$; AS2.2, $2.9 \mathrm{~g} \mathrm{~L}^{-1}$ and Stm3LR3, $4.4 \mathrm{~g} \mathrm{~L}^{-1}$. Wild-type $C_{X}$ was $2.6 \mathrm{~g} \mathrm{~L}^{-1}$.

Several experiments have been performed with cell wall-less antenna size mutants (TLA1 CW- (Polle et al. 2003) and Stm3LR3 (Mussgnug et al. 2007)) because cell wall removal was required in the process of creating these mutants. However, cultivation was cumbersome. Cell wall-less mutants were found to be fragile in a well-mixed photobioreactor. This fragility also created difficulty when determining the biomass dry weight concentration utilizing vacuum filtration. Due to cell breakage, less biomass was retained on the fiber filters (i.e., biomass was lost), and there was greater variation in the dry weight measurement. However, for the cell wall-less mutant Stm3LR3, we managed to perform 2 successful experiments by minimizing the hydrodynamic forces in the photobioreactor (i.e. a lower gas flow). Fig. 2.8A illustrates the results of these experiments, which are similar to the results established for the wild-type and the other mutants. The measured biomass concentrations and dilution rates varied significantly for the two replicate experiments; the analysis of the accompanied absorption spectra revealed an increased pigmentation for the culture with the lowest biomass concentration (Fig. 2.8B). The productivity was not markedly affected by the shift in pigmentation. 
$r_{x}\left(g^{-2} h^{-1}\right) \square D\left(d^{-1}\right)$

$\mathrm{C}_{\mathrm{x}}\left(\mathrm{g} \mathrm{L}^{-1}\right)$ पIIA $\mathrm{C}_{\mathrm{x}}\left(\mathrm{g} \mathrm{L}^{-1}\right)$
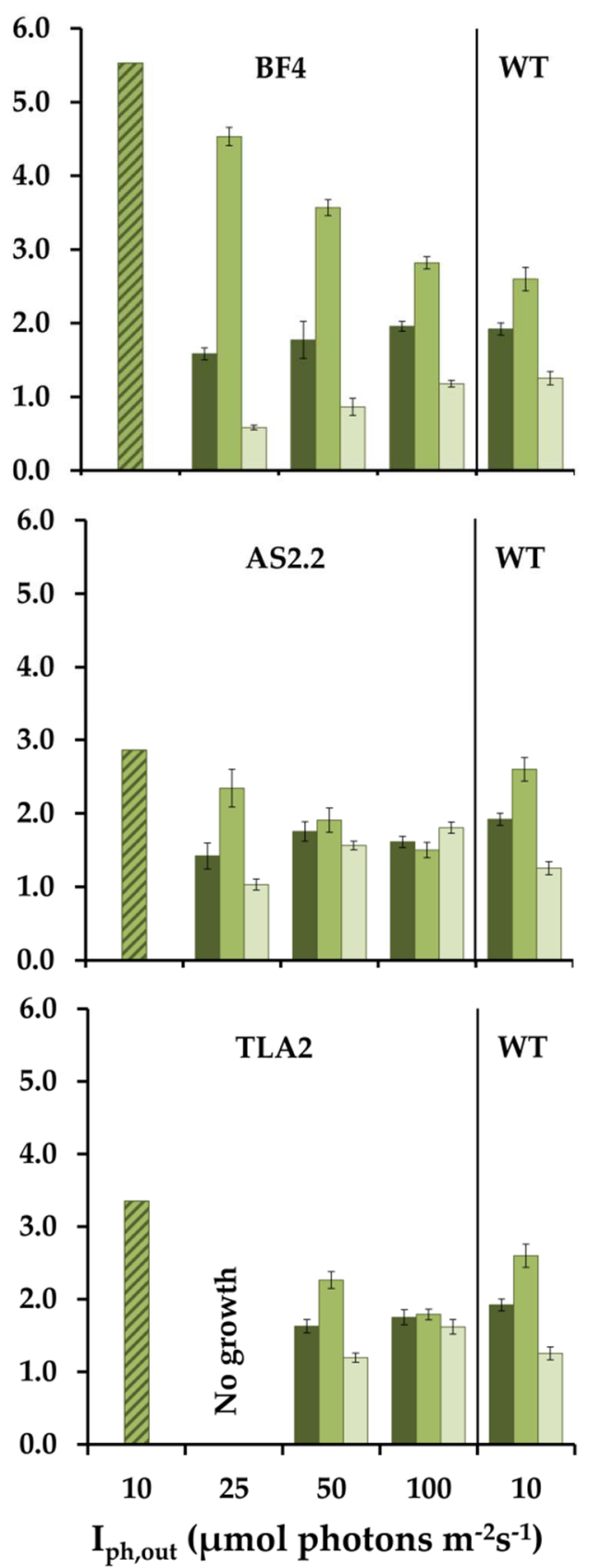

Fig. 2.7 Areal biomass productivity $\left(r_{x}\right)$, biomass concentration $\left(C_{x}\right)$, and dilution rate $(D)$ at an $I_{p h, i n}$ of $1500 \mu \mathrm{mol} \mathrm{m} \mathrm{m}^{-2} \mathrm{~s}^{-1}$ and different $I_{\text {ph,out. }} C_{x}{ }^{\prime}$ is the extrapolated biomass concentration of a mutant strain if it would have been cultivated at the same $I_{p h, o u t}(10$ $\left.\mu \mathrm{mol} \mathrm{m}^{-2} \mathrm{~s}^{-1}\right)$ as the wild-type. For mutants, each bar represents the average of $n \geq 4$ data points $\pm S D$ that were measured on a daily basis within one reactor experiment. For wild-type, the bars represent the average of $n=20$ data points \pm pooled $S D$ that were measured on a daily basis within 4 different reactor experiments. 

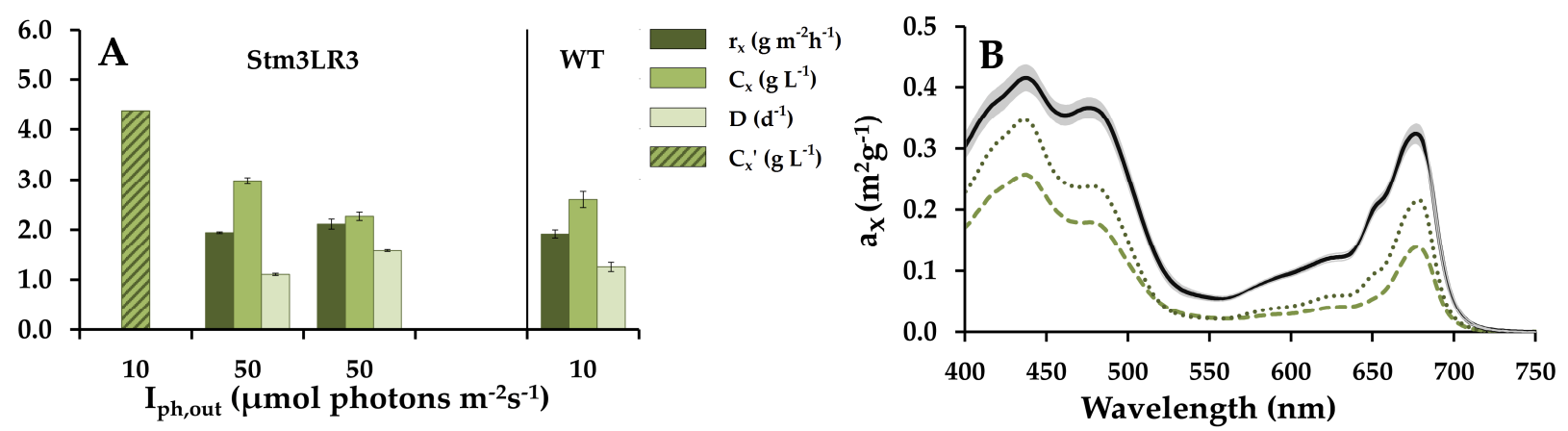

Fig. 2.8 (A) Areal biomass productivity $\left(r_{x}\right)$ at various outgoing light intensities ( $\left.I_{\text {ph,out }}\right)$. For Stm3LR3, each bar represents the average of $n \geq 4$ data points $\pm S D$ that were measured on a daily basis within one reactor experiment. For the wild-type, see Fig. 2.7 (B) : Wavelength dependent biomass dry weight specific optical cross section $\left(a_{x}\right)$ of the Stm3LR3 culture with a $C_{x}$ of $3.0 \mathrm{~g} \mathrm{~L}^{-1}$ (dashed line) and with a $C_{x}$ of $2.3 \mathrm{~g} \mathrm{~L}^{-1}$ (dotted line), as shown in (a). Lines represent the average of 4 reactor experiments $\pm S D$ (wildtype) and a single reactor experiment (Stm3LR3).

Several experiments have been performed with cell wall-less antenna size mutants (TLA1 CW- (Polle et al. 2003) and Stm3LR3 (Mussgnug et al. 2007)) because cell wall removal was required in the process of creating these mutants. However, cultivation was cumbersome. Cell wall-less mutants were found to be fragile in a well-mixed photobioreactor. This fragility also created difficulty when determining the biomass dry weight concentration utilizing vacuum filtration. Due to cell breakage, less biomass was retained on the fiber filters (i.e., biomass was lost), and there was greater variation in the dry weight measurement. However, for the cell wall-less mutant Stm3LR3, we managed to perform 2 successful experiments by minimizing the hydrodynamic forces in the photobioreactor (i.e. a lower gas flow). Fig. 2.8A illustrates the results of these experiments, which are similar to the results established for the wild-type and the other mutants. The measured biomass concentrations and dilution rates varied significantly for the two replicate experiments; the analysis of the accompanied absorption spectra revealed an increased pigmentation for the culture with the lowest biomass concentration (Fig. 2.8B). The productivity was not markedly affected by the shift in pigmentation.

In Fig. 2.9, the performance of the strains is depicted as the biomass yield on light energy $\left(Y_{x / p h}\right)$. For all mutant strains that were cultivated under different $I_{p h, o u t}$, only the most significant measured yield is indicated. $Y_{X / p h}$ can be expressed based on incident light or on absorbed light, the latter being corrected for the outgoing light intensity (see eq. 6 
and 7). In the latter case, strain performance is slightly higher than when expressed on an incident light basis, especially for mutants cultivated at a high $I_{p h \text { out }}$. If a mutant showed a similar $r_{x}$ but higher $I_{p h \text {,out }}$ (e.g. $100 \mu \mathrm{mol} \mathrm{m} \mathrm{m}^{-2} \mathrm{~s}^{-1}$ versus $10 \mu \mathrm{mol} \mathrm{m}{ }^{-2} \mathrm{~s}^{-1}$ nonabsorbed light) compared to the wild-type strain, its $Y_{x / p h}$ on an absorbed light basis will be higher since less light was absorbed to achieve the same productivity.

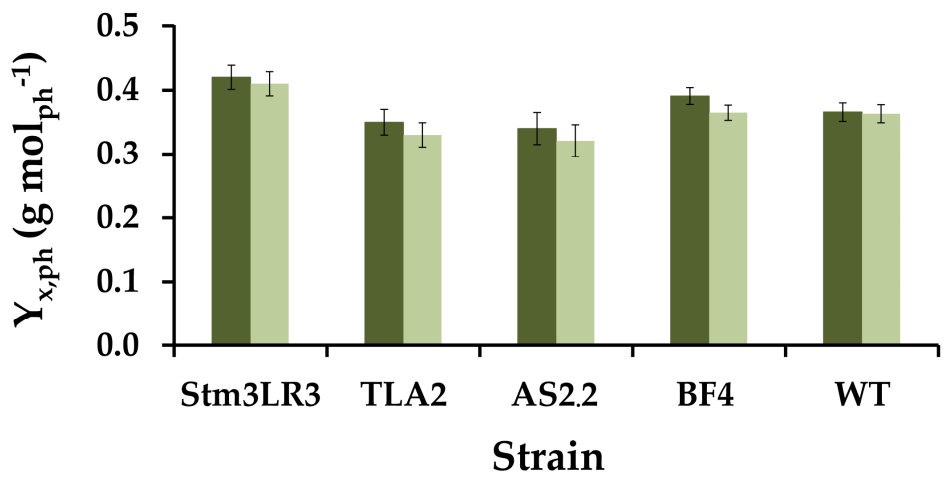

Fig. 2.9 Biomass yields on light energy $\left(Y_{x, p h}\right)$ of antenna size mutants and the wild-type. $Y_{x, p h}$ is indicated on an incident light energy basis (light bars) and on an absorbed light energy basis (dark bars). For the mutants, each bar represents the average of $n \geq 4$ data points $\pm S D$ that were measured on a daily basis within one reactor experiment. For the wild-type, the bars represent the average of $n=20$ data points \pm pooled SD that were measured on a daily basis within 4 reactor experiments. For results based on absorbed light energy, the outgoing light intensity for each strain was: Stm3LR3, $50 \mu \mathrm{mol} \mathrm{m}{ }^{-2} \mathrm{~s}^{-1}$; TLA2, $100 \mu \mathrm{mol} \mathrm{m}^{-2} \mathrm{~s}^{-1}$; AS2, $50 \mu \mathrm{mol} \mathrm{m}^{-2} \mathrm{~s}^{-1}$; BF4, $100 \mathrm{\mu mol} \mathrm{m}^{-2} \mathrm{~s}^{-1}$; WT, $10 \mu \mathrm{mol} \mathrm{m}{ }^{-2} \mathrm{~s}^{-1}$.

An important observation was that all mutant cultures were ascertained to be more sensitive to photodamage than the wild-type. Growth of the mutants sometimes ceased when the light intensity was increased too much. This resulted in very low dilution rates or no dilution at all. These cultures often recovered when the light intensity was reduced, however, in some cases, the culture collapsed while the wild-type strain cultivated under identical conditions did not exhibit any sign of stress.

In additional experiments, we compared the performance of three mutants with the wild-type at an $I_{p h, i n}$ of $850 \mu \mathrm{mol} \mathrm{m} \mathrm{s}^{-2} \mathrm{~s}^{-1}$ and an $I_{p h, o u t}$ of $50 \mu \mathrm{mol} \mathrm{m}^{-2} \mathrm{~s}^{-1}$ for the mutants and $10 \mu \mathrm{mol} \mathrm{m}{ }^{-2} \mathrm{~s}^{-1}$ for the wild-type strain (Fig. 2.10). Mutants did not exhibit a higher $r_{x}$ than the wild-type $\left(1.5 \mathrm{~g} \mathrm{~m}^{-2} \mathrm{~h}^{-1}\right)$ but attained similar values $\left(1.2\right.$ to $\left.1.4 \mathrm{~g} \mathrm{~m}^{-2} \mathrm{~h}^{-1}\right)$. 


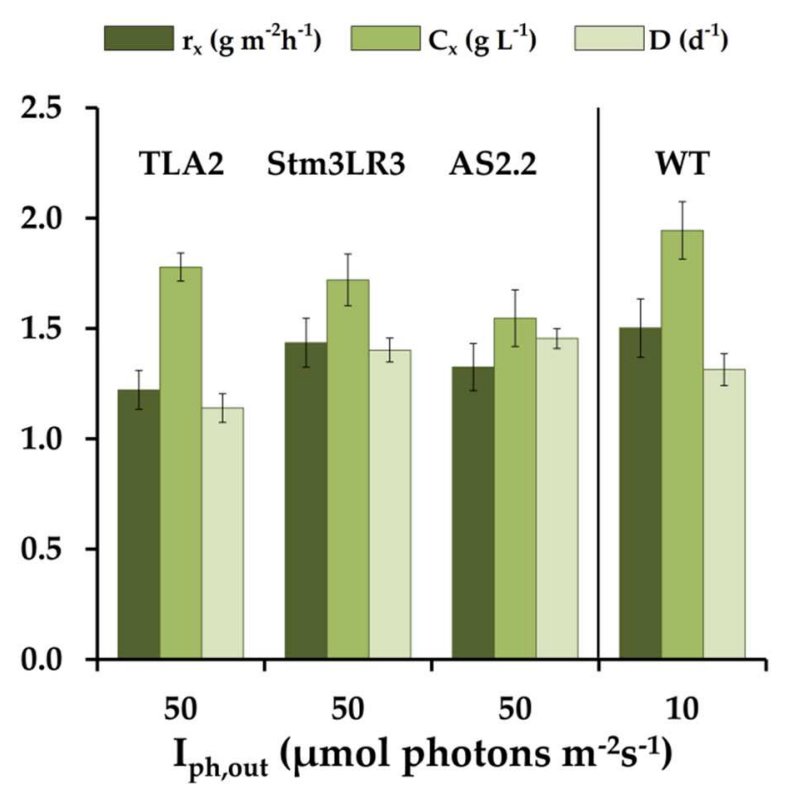

Fig. 2.10 Areal biomass productivity at an ingoing light intensity of $850 \mu \mathrm{mol} \mathrm{m} \mathrm{m}^{-2} \mathrm{~s}^{-1}$ and an outgoing light intensity of $50 \mu \mathrm{mol} \mathrm{m} \mathrm{m}^{-2} \mathrm{~s}^{-1}$ for the mutants and $10 \mu \mathrm{mol} \mathrm{m} \mathrm{m}^{-2} \mathrm{~s}^{-1}$ for wild-type. For the mutants, the error bars indicate the standard deviation $(n \geq 5)$ of a single reactor experiment. For the wild-type, the bars represent the average of $n=11$ data points \pm pooled SD that were measured on a daily basis within 2 reactor experiments.

\subsection{Discussion}

Wild-type Chlamydomonas cells are easily oversaturated by high light intensities. We measured biomass yields on light $\left(Y_{x / p h}\right)$ of $0.37 \mathrm{~g} \mathrm{~mol}_{\mathrm{ph}}{ }^{-1}$ at an $I_{p h, i n}$ of $1500 \mu \mathrm{mol} \mathrm{m} \mathrm{s}^{-2} \mathrm{~s}^{-1}$ and $0.51 \mathrm{~g} \mathrm{~mol}_{\mathrm{ph}}{ }^{-1}$ at an $I_{p h, i n}$ of $850 \mu \mathrm{mol} \mathrm{m}^{-2} \mathrm{~s}^{-1}$. A biomass yield on light energy of $1.25 \mathrm{~g}$ mol $_{\mathrm{ph}^{-1}}$ can be attained at a very low incident light intensity (Kliphuis et al. 2012) when photosynthesis is limited by light and heat dissipation is minimal or absent. Theoretically, the difference between this value and the yields we measured determines the scope for improvement of the biomass productivity employing genetically engineered strains with truncated antenna complexes. By preventing energy losses resulting from the oversaturation of the photosystems, the excess light energy can potentially be converted to algal biomass, increasing the areal biomass productivity of the culture.

The optimal biomass concentration to maximize biomass productivity is found when, at the back of the reactor, the net photosynthesis rate is equal to zero (Takache et al. 2010). The light intensity at this condition is the photosynthetic compensation point. For Chlamydomonas reinhardtii, the photosynthetic compensation point was found to be approximately $10 \mu \mathrm{mol} \mathrm{m} \mathrm{m}^{-2} \mathrm{~s}^{-1}$ (Takache et al. 2010, Vejrazka et al. 2013). This value, however, does not represent a sharp optimum. During the experiments with the wildtype cells described in this paper, an outgoing light intensity of $10 \mu \mathrm{mol} \mathrm{m}^{-2} \mathrm{~s}^{-1}$ was 
assumed to result in the highest productivity. Since antenna size mutants have less light absorption capacity, their photosynthetic compensation point will be higher than that of the wild-type. For this reason and with no reference data available, maximal mutant productivity was sought for in a range of outgoing light intensities of $25-100 \mu \mathrm{mol} \mathrm{m}{ }^{-2} \mathrm{~s}^{-1}$.

We used our model to estimate the areal biomass productivity of cells with a high (wildtype) or low (antenna size mutants) pigment content under mass culture conditions. The predicted $r_{x}$ for the wild-type strain $\left(1.7 \mathrm{~g} \mathrm{~m}^{-2} \mathrm{~h}^{-1}\right)$ is very much in accordance with the experimentally achieved $r_{x}\left(1.9 \mathrm{~g} \mathrm{~m}^{-2} \mathrm{~h}^{-1}\right)$. In contrast to the expectations, the experimentally attained results for the mutants did not meet the model predictions. Two important assumptions were made that could have led to an overestimation of the areal biomass productivity. Foremost, $a_{x}$ is presupposed to be constant at any position in the photobioreactor while, in reality, the spectrum at the back of the reactor primarily consists of green light, which can hardly be absorbed by green algae and therefore results in a lower photosynthetic rate at that specific position of the reactor. The second assumption is that there is no backscattering in the photobioreactor. Otherwise stated, all photons are absorbed by the culture except for the minimal amount of light leaving the reactor at the back.

Overall, mutant cultures did not exhibit the estimated doubling in productivity in comparison to the wild-type cultures. Mutant cultures contained more cell debris than the wild-type culture, probably as a consequence of a higher cell death rate. This could also explain why mutant cultures displayed a greater tendency to foam and were easily overgrown by bacteria. Microscopic analysis revealed that a small fraction of the mutant cells was colourless, most plausibly the result of severe photodamage. During the cultivations, both biomass concentration and specific growth rate fluctuated more than that of the wild-type, and mutants were more sensitive to abrupt light shifts than the wild-type strain.

For three of the mutants, the areal biomass productivity increased at an increasing higher outgoing light intensity (from $25 \mu \mathrm{mol} \mathrm{m} \mathrm{m}^{-2} \mathrm{~s}^{-1}$ to $100 \mu \mathrm{mol} \mathrm{m}^{-2} \mathrm{~s}^{-1}$ ). This signifies that antenna size mutants do, indeed, require more light to achieve the same photosynthetic activity as the wild-type, for which the optimal $I_{p h, o u t}$ was estimated to be only $10 \mu \mathrm{mol} \mathrm{m}{ }^{-2} \mathrm{~s}^{-1}$. In some cases, the applied outgoing light intensities may have been 
too low for cells with a low pigment content, which explains why mutants could not be cultivated at an outgoing light intensity of $10 \mu \mathrm{mol} \mathrm{m}^{-2} \mathrm{~s}^{-1}$. We hypothesize that, under the resulting light regime, respiration became a significant factor balancing the photosynthetic rate. Stated differently, the antenna size mutants probably experienced a dark zone at the back of the reactor in which the net photosynthetic rate was negative, and this even led to the absence of net growth in the photobioreactor at very low levels of $I_{p h \text { out }}\left(<10 \mu \mathrm{mol} \mathrm{m} \mathrm{m}^{-2} \mathrm{~s}^{-1}\right.$ ). However, even with higher outgoing light intensities, cultivation of mutants did not fulfil the expectation of improved biomass productivity.

For the cell wall-less mutants, it is plausible that shear stress caused by hydrodynamic forces in the photobioreactor resulted in breakage of the cells, an increase in the amount of cell debris, which subsequently led to an uncontrolled growth of bacteria. Unstable cultures and unreliable biomass concentrations evidenced that cell wall-less mutants were unsuitable for our experimental conditions. A remarkable finding was that, under the same light regime in two reactor experiments, different biomass concentrations were attained for the Stm3LR3 mutant. This can only be explained by an increased optical cross section of the culture. Otherwise stated, this mutant possibly lost part of its phenotype after the first reactor experiment was performed.

It is often erroneously supposed that the increased light penetration in antenna size mutant cultures is a major factor for improving the biomass productivity. However, in order to maximize productivity under mass culture conditions, light penetration is a parameter that is automatically controlled by adjusting the biomass concentration. This results in a photobioreactor with a similar light gradient as the wild-type culture but with a higher biomass concentration. Mutants could not, however, be cultivated in the outgoing light intensity assumed to be optimal for the wild-type strain. The photosynthetic compensation point of mutants appears to be higher than that of the wild-type strain. This statement is substantiated by our experimental data. An increased light transmittance (i.e., by applying a higher outgoing light intensity) in an antenna size mutant culture was ascertained to be a requirement for maximizing productivity and not a feature of antenna size mutants with a higher transparency.

The antenna size mutants were found to absorb less light per cell and, as a consequence, could be cultivated at higher biomass concentrations than the wild-type depending upon 
the actual optical cross section of the antenna size mutant and the selected $I_{p h, o u t}$. In the event of a healthy culture, the lower light absorption rate per cell should have resulted in a lower dissipation rate and, therefore, a higher biomass productivity and biomass yield on light energy. We did not observe an increased biomass yield on light energy. The attained results suggest that the modifications to the antenna complexes resulted in complications that ultimately eliminated the anticipated beneficial effect of a truncated antenna complex. Therefore, the most plausible explanation for the reduced fitness of the mutants during long term experiments is that the modified antenna complexes lost essential regulatory functions involved in the distribution, conversion, or dissipation of light energy.

Most antenna size mutants are deficient in chlorophyll $\mathrm{a} / \mathrm{b}$ binding proteins and the subunits necessary for assembling the peripheral components of the light harvesting antenna. However, many of these binding proteins and subunits have been evidenced to play a role in photoprotection mechanisms and energy distribution. Loss of CP29, a binding protein, leads to impaired state transitions (Tokutsu et al. 2009). LHCSR3, a Chl $\mathrm{a} / \mathrm{b}$ binding stress-related member of the LHC protein superfamily, is of high importance for cell survival at high light intensities as it is essential for the performance of heat dissipation (qE) (Tokutsu et al. 2013). Cells survival after switching from a low to high light intensity has been found to be lower when LHCSR genes were lacking (Peers et al. 2009). Moreover, several antenna proteins are known to function in photoprotective mechanisms in microalgae and higher plants (Alboresi et al. 2009). For example, recently it was shown that the LHCII protein LHCBM9 is highly important for photoprotection during prolonged stress conditions in C. reinhardtii (Grewe et al. 2014). Antenna size mutants have also been generated in which binding proteins are not down regulated in a direct way (Perrine et al. 2012). In these mutants, the chlorophyll b formation by the enzyme chlorophyllide a oxygenase (CAO) is inhibited. It seems that this would be a more safe approach. However, there is certain evidence indicating that chlorophyll $\mathrm{b}$ functions as an antenna size regulator and possesses an additional important structural role in protein-pigment complexes (Tanaka et al. 2000). This signifies that a reduction of Chl b would, in any case, be accompanied with a decrease in antennae proteins involved in photoprotection. 
Other researchers have encountered similar issues with antenna size mutants. Although Cyclotella sp. mutants demonstrated a higher light saturation level, cultivation was described as less stable than the wild-type, and mutant cultures were found to collapse within ten days (Huesemann et al. 2009). Less resistance against high light intensities is provided as a possible explanation. In another study, antenna mutants of the cyanobacterium Synechocystis lacking the complete light harvesting antenna were found to have a lower robustness and fitness than the wild-type (Kwon et al. 2013). This strain could not be cultivated at light intensities higher than $300 \mu \mathrm{mol} \mathrm{m} \mathrm{m}^{-2} \mathrm{~s}^{-1}$, and proteomic analysis revealed an increased concentration of enzymes that are normally synthesized in stress situations. This result shows that, for cyanobacteria, at least some light harvesting antenna subunits are essential for cell survival at high light intensities. Another mutant created by the same researchers which lacked only specific antenna subunits did not exhibit this stress-phenotype. However, these mutants had not been cultivated at a high light intensity where the need for photoprotection is highest and the effects of photosaturation are maximal. In a different study regarding a Synechocystis mutant, researchers describe phycobilisome reduction as a suboptimal strategy to improve culture productivity as the modifications lead to changes in many critical components that are essential for cell function which, consequently, results in decreased productivity (Liberton et al. 2013). Distinguishing essential photoprotection subunits from subunits that are strictly involved in light harvesting and subsequently maintaining these essential subunits in the mutant selection procedure might be the crucial element to the realization of the ideal antenna size mutant (Peers et al. 2009, Bonente et al. 2011). The productivity increase achieved by genetically engineering Chlamydomonas perigranulata appears to be the only successful experiment so far that was conducted under mass cultivation conditions (Nakajima et al. 2001). The LHC-I mutant demonstrated a 1.5 times higher productivity than the wild-type at an $I_{p h, i n}$ of $2000 \mu \mathrm{mol}$ $\mathrm{m}^{-2} \mathrm{~s}^{-1}$. The applied light regime, however, is inadequately described, which raises the question whether the wild-type, being the negative control, was cultivated under optimal conditions. It would be interesting to study this specific mutant in more detail under well-defined light conditions in a turbidostat controlled reactor.

During their generation, most antenna size mutant strains were selected based solely on their low pigment content. Therefore, to prevent loss of photoprotection in the future, in 
addition to a low absorptive capacity, high light resistance of individual cells should be a criterion during the selection procedure. Screening for high light resistance is easily implemented and will lead to strains that are much more appropriate as starter cultures for further analysis of their biomass productivity under mass culture conditions. Being aware that algae have the natural capability to halve their pigment content at high light conditions, there must be ways to achieve this by genetic engineering without loss of photoprotection. The generation and evaluation of antenna size mutants is a process in which researchers from various disciplines are involved, each with their own focus and expertise. In consideration of this, we would like to emphasize that the engineering aspects of optimizing productivity of antenna size mutants should not be underestimated. Accurate and reliable productivity measurements under simulated mass culture conditions are essential for a viable evaluation of mutant performance. This requires knowledge of the optimal conditions to maximize productivity of microalgae cultures and how these conditions change in the event of pigment reduction. In addition, a continuous photobioreactor system combined with a well-defined light regime is of critical importance to create and maintain optimal cultivation conditions. By modelling microalgal productivity using experimental data from simulated mass culture conditions, a more solid base is obtained to describe the future potential and both theoretical and practical implications of antenna size reduction. In this perspective, it is advisable to involve bioprocess engineers in an early stage of antenna size mutant creation process as well. By combining expertise of molecular biology and bioprocess engineering, an improved understanding of the requirements for creating the most superior antenna size mutant will be obtained.

\subsection{Conclusions}

In this study, we present the areal biomass productivities of antenna size mutants quantified under simulated mass culture conditions. The long-term photobioreactor experiments under turbidostatic control combined with biomass dry weight measurements and a well-defined light regime provided a reliable basis to study culture productivity. A fixed incident light intensity of $1500 \mu \mathrm{mol} \mathrm{m} \mathrm{m}^{-2} \mathrm{~s}^{-1}$ guaranteed oversaturation of the wild-type strain and provided the conditions in which antenna size mutant cultures were anticipated to demonstrate a higher light use efficiency and 
doubling of productivity. The antenna mutants we tested were confirmed to have a lower light absorption capacity than the wild-type. The mutants, however, did not exhibit the expected increase in biomass productivity under mass culture conditions. The highest mutant productivity values were comparable $\left(1.8-2.1 \mathrm{~g} \mathrm{~m}^{-2} \mathrm{~h}^{-1}\right)$ to that of wild-type productivity $\left(1.9 \mathrm{~g} \mathrm{~m}^{-2} \mathrm{~h}^{-1}\right)$. The biomass yield on light energy ranged between 0.33 and $0.41 \mathrm{~g} \mathrm{~mol}_{\mathrm{ph}^{-1}}$ for the mutants and was $0.36 \mathrm{~g} \mathrm{~mol}_{\mathrm{ph}^{-1}}$ for the wild-type strain. Under turbidostat control, mutant cultures required a higher outgoing light intensity than the wild-type culture in order to achieve maximal areal productivity. Observations indicated a reduced fitness of the mutant cultures caused by a high light intensity. This might be explained by a higher susceptibility to photodamage compared to the wild-type strain. The principle of antenna size reduction is a promising strategy to increase productivity. However, for future generations of antenna size mutants, in addition to reducing the light absorptive capacity of the cells, emphasis must be placed on tolerance to high light conditions that are evident in mass culture conditions. 


\section{Appendix A: Microalgae growth model and photobioreactor productivity}

A simple kinetic model was constructed to describe microalgae productivity as a function of the light intensity. The model is based on two compartments. In the first compartment, the chloroplast, there is photosynthetic production of 3-carbon sugars (triose) symbolized by the 1-carbon sugar equivalent $\mathrm{CH}_{2} \mathrm{O}$. This sugar production rate in the chloroplast $\left(q^{c} \mathrm{CH} 2 \mathrm{O}, \mathrm{mol}_{\mathrm{CH} 2 \mathrm{O}} \mathrm{mol}_{\mathrm{x}}{ }^{-1} \mathrm{~s}^{-1}\right)$ is dependent upon light intensity $I_{p h}$ and described by the hyperbolic tangent model of Jassby and Platt (Jassby et al. 1976):

$$
q_{C H 2 O}^{c}=q_{C H 2 O, m}^{c} \cdot \tanh \left(\frac{I_{p h} \cdot a_{x} \cdot Y_{C H 2 O / p h, m}}{q_{C H 2 O, m}^{c}}\right)
$$

Where $I_{p h}$ is the PAR photon flux density $\left(\operatorname{mol}_{\mathrm{ph}} \mathrm{m}^{-2} \mathrm{~s}^{-1}\right), q^{c} C H 20, m$ is the maximal sugar production rate $\left(\mathrm{mol}_{\mathrm{CH} 2 \mathrm{O}} \mathrm{mol}_{\mathrm{x}}^{-1} \mathrm{~S}^{-1}\right)$ in the chloroplast; $Y_{C H 2 O / p h, m}$ is the maximal yield of sugar on light energy $\left(\operatorname{mol}_{\mathrm{CH} 2 \mathrm{O}} \mathrm{mol}_{\mathrm{ph}}{ }^{-1}\right)$; and $a_{x}$ is the spectrally averaged optical cross section of the microalgae $\left(\mathrm{m}^{2} \mathrm{~mol}_{\mathrm{x}}{ }^{-1}\right) \cdot a_{x}$ can be calculated according to the following equation:

$a_{x}=\sum_{\lambda=400}^{\lambda=700} a_{x, \lambda} \cdot E_{n, \lambda} \cdot \Delta \lambda$

In which $a_{x, \lambda}$ is the optical cross section at wavelength $\lambda . E_{n, \lambda}\left(\mathrm{nm}^{-1}\right)$ represents the normalized spectral distribution of the light source (Fig. 2.2). It is the fraction of photons in the PAR region in a $1 \mathrm{~nm}$ interval at specific $\lambda$.

The cell minus the chloroplast comprises the second compartment in which the 3carbon sugar is used to build new biomass at a specific growth rate $\mu$. Another part of the sugar is respired in the mitochondria to provide energy (ATP) to support the growth reactions and to fulfill the maintenance requirements. The consumption of sugar in the chloroplast can be described by Pirt's Law (Pirt 1965), resulting in the following relation:

$\mu=\left(q_{C H 2 O}^{c}-m_{C H 2 O}\right) \cdot Y_{x / C H 2 O}$

and:

$\mu_{m}=\left(q_{C H 2 O, m}^{c}-m_{C H 2 O}\right) \cdot Y_{x / C H 2 O}$ 
In these equations, $m_{\mathrm{CH} 2 \mathrm{O}}$ is the biomass specific maintenance rate $\left(\operatorname{mol}_{\mathrm{CH} 2 \mathrm{O}} \mathrm{mol}_{\mathrm{x}}{ }^{-1} \mathrm{~s}^{-1}\right)$; $Y_{x / \mathrm{CH} 2 \mathrm{O}}$ is the biomass yield on 3-carbon sugar $\left(\operatorname{mol}_{\mathrm{x}} \operatorname{mol}_{\mathrm{CH} 2 \mathrm{O}^{-1}}\right)$; and $\mu_{m}$ is the biomass specific growth rate $\left(\mathrm{s}^{-1}\right)$.

The biomass on yield on light energy $\left(\mathrm{mol}_{\mathrm{x}} \mathrm{mol}_{\mathrm{ph}}{ }^{-1}\right)$ can now be calculated as follows:

$Y_{x / p h}=\frac{\mu}{q_{p h}}$

Here, $q_{p h}$ is the specific light absorption rate $\left(\mathrm{mol}_{\mathrm{ph}} \operatorname{mol}_{\mathrm{x}}^{-1} \mathrm{~s}^{-1}\right)$, which is defined as:

$q_{p h}=a_{x} \cdot I_{p h}$

The light intensity at which $\mu$ is equal to zero (i.e. where photosynthetic sugar production is compensated by maintenance associated sugar consumption) is referred to as the photosynthetic compensation point $\left(I_{p h, c}\right)$. By numerical integration of $Y_{x / p h}$ from $I_{p h}=I_{p h, c}$ to $I_{p h}=1500 \mu \mathrm{mol} \mathrm{m} \mathrm{m}^{-2} \mathrm{~s}^{-1}$, the maximal biomass productivity per illuminated surface area $\left(r_{x}\right)$ is obtained in $\mathrm{g} \mathrm{m}^{-2} \mathrm{~h}^{-1}$ (eq. A7).

$r_{x}=3600 \cdot 10^{-6} \cdot \sum_{I_{p h, c}}^{I_{p h, i n}} Y_{x / p h} \cdot \Delta I_{p h}$

Table A1 Overview of the model parameters.

\begin{tabular}{|c|c|c|c|c|}
\hline Parameter & Value & Unit & Description & References \\
\hline$\mu_{m}$ & 0.132 & $h^{-1}$ & $\begin{array}{l}\text { maximal biomass specific growth rate; } \\
\text { assumed to be equal for WT and BF4 }\end{array}$ & (Janssen et al. 2000) \\
\hline$q \varepsilon_{\mathrm{CH} 20, \mathrm{~m}}$ & $6.17 \cdot 10^{-5}$ & $\operatorname{mol}_{\mathrm{CH} 2 \mathrm{O}} \mathrm{mol}_{\mathrm{x}}^{-1} \mathrm{~S}^{-1}$ & $\begin{array}{l}\text { maximal 3-carbon sugar production } \\
\text { rate; calculated from } \mu_{m} \text { using eq. A4. }\end{array}$ & \\
\hline$m_{\mathrm{CH} 2 \mathrm{O}}$ & $3.5 \cdot 10^{-6}$ & $\operatorname{mol}_{\mathrm{CH} 2 \mathrm{O}} \operatorname{mol}_{\mathrm{x}}^{-1} \mathrm{~S}^{-1}$ & biomass specific maintenance rate & (Kliphuis et al. 2012) \\
\hline$Y_{X / C H 2 O}$ & 0.63 & $\operatorname{mol}_{\mathrm{x}} \operatorname{mol}_{\mathrm{CH} 2 \mathrm{O}^{-1}}$ & $\begin{array}{l}\text { biomass yield on 3-carbon sugar using } \\
\text { ammonia as N-source }\end{array}$ & (Kliphuis et al. 2012) \\
\hline$a_{x}(\mathrm{WT})$ & 6.2 & $\mathrm{~m}^{2} \mathrm{~mol}_{\mathrm{x}}^{-1}$ & $\begin{array}{c}\text { spectrally averaged optical cross } \\
\text { section }\end{array}$ & This paper \\
\hline$a_{x}$ (Mutant) & 1.9 & $\mathrm{~m}^{2} \mathrm{~mol}_{\mathrm{x}}^{-1}$ & $\begin{array}{c}\text { spectrally averaged optical cross } \\
\text { section }\end{array}$ & This paper \\
\hline$Y_{C H 2 O / p h, m}$ & 0.10 & $\operatorname{mol}_{\mathrm{CH} 2 \mathrm{O}} \mathrm{mol}_{\mathrm{ph}^{-1}}$ & $\begin{array}{c}\text { maximal yield of 3-carbon sugar on } \\
\text { light energy }\end{array}$ & (Blanken et al. 2013) \\
\hline$M_{x}$ & 24 & $\mathrm{~g} \mathrm{~mol}_{\mathrm{x}}^{-1}$ & $\begin{array}{l}\text { Biomass dry weight to C-mol } \\
\text { conversion factor for algae }\end{array}$ & (Duboc et al. 1999) \\
\hline
\end{tabular}




\section{Appendix B: Light intensity distribution over reactor surface}

Table B1 Incident light intensity ( $\mu \mathrm{mol}$ photons $\mathrm{m}^{-2} \mathrm{~s}^{-1}$ ) distribution over the reactor surface. The light was measured at 28 points evenly distributed over the light exposed surface of the front glass panel of the culture chamber. For this measurement, the average light intensity was $1501 \mu \mathrm{mol} \mathrm{m}^{-2} \mathrm{~s}^{-1}$.

\begin{tabular}{cccc}
\hline 1235 & 1429 & 1352 & 1286 \\
1464 & 1665 & 1656 & 1409 \\
1683 & 1837 & 1737 & 1739 \\
1586 & 1882 & 1953 & 1571 \\
1601 & 1738 & 1670 & 1438 \\
1383 & 1568 & 1591 & 1177 \\
1001 & 1135 & 1144 & 1099 \\
\hline
\end{tabular}




\section{Appendix C: Calculation of the extrapolated biomass concentration $C_{x}{ }^{\prime}$ using the attenuation coefficient $K_{x}$}

For each mutant, the spectrally averaged attenuation coefficient $\left(K_{x}, \mathrm{~m}^{2} \mathrm{~g}^{-1}\right)$ of the experiment with the darkest light regime (i.e. lowest $I_{p h, o u t}$ ) was calculated. This attenuation coefficient $K_{X}$ was determined by measuring the light transmittance in the photobioreactor by measuring the $I_{p h, i n}$ and $I_{p h \text {,out }}$ using a PAR light meter. This attenuation coefficient $K_{x}$, therefore, also includes the effect of light scattering as it occurs in the photobioreactor. The coefficient $K_{x}$ is fundamentally different from the spectrally averaged optical cross section $\left(a_{x}\right)$ which only reflects true light absorption. $K_{x}$ was calculated using the equations indicated below in which $d(\mathrm{~m})$ is the light path of the photobioreactor.

First, $K_{X}$ is calculated using eq. $\mathrm{C} 1$ which was obtained by rearranging eq. C2, LambertBeer's law equation.

$K_{x}=\frac{\ln \frac{I_{p h, o u t}}{I_{p h, i n}}}{-C_{x} \cdot d}$

$I_{p h, o u t}=I_{p h, i n} \cdot e^{-C_{x} \cdot K_{x} \cdot d}$

With $K_{X}$ known, $C_{X}{ }^{\prime}$ at $I_{p h, o u t}=10 \mu \mathrm{mol} \mathrm{m}{ }^{-2} \mathrm{~s}^{-1}$ can be estimated using eq. C3, which was also obtained by rearranging eq. $\mathrm{C} 2$.

$C_{x}^{\prime}=\frac{\ln \frac{I_{\text {ph,out }}}{I_{p h, i n}}}{-K_{x} \cdot d}$ 


\section{Appendix D: Light measurements and experimental data}

Table D1 Overview of light measurements and experimental results using wild-type strains. The data in the grey colored columns was not used for comparison with mutant strains because of the influence of agglomerate formation on the biomass productivity.

\begin{tabular}{|c|c|c|c|c|c|c|c|c|c|c|}
\hline Experiment & 1 & 2 & 3 & 4 & 5 & 6 & 7 & 8 & 9 & 10 \\
\hline Wild type strain & 1690 & 1690 & 1690 & 1690 & 1690 & 1690 & 1690 & 124 & 1690 & 1690 \\
\hline Iph,in $\left(\mu \mathrm{mol} \mathrm{m} \mathrm{m}^{-2} \mathrm{~s}^{-1}\right)$ & 1524 & 1477 & 1491 & 1409 & 1504 & 1485 & 1489 & 1508 & 834 & 833 \\
\hline Iph,out $\left(\mu \mathrm{mol} \mathrm{m}{ }^{-2} \mathrm{~s}^{-1}\right)$ & 9 & 9 & 9 & 9 & 9 & 11 & 10 & 11 & 9 & 10 \\
\hline$C_{x}\left(\mathrm{~g} \mathrm{~L}^{-1}\right)$ & 2.8 & 2.6 & 2.8 & 2.7 & 2.5 & 2.6 & 2.5 & 2.8 & 2.0 & 1.9 \\
\hline SD & 0.07 & 0.08 & 0.06 & 0.31 & 0.05 & 0.09 & 0.07 & 0.10 & 0.15 & 0.11 \\
\hline$D\left(\mathrm{~d}^{-1}\right)$ & 1.4 & 1.3 & 1.5 & 1.2 & 1.2 & 1.4 & 1.6 & 1.4 & 1.3 & 1.3 \\
\hline SD & 0.04 & 0.04 & 0.07 & 0.18 & 0.05 & 0.04 & 0.05 & 0.04 & 0.06 & 0.09 \\
\hline$r_{x}\left(\mathrm{~g} \mathrm{~m}^{-2} \mathrm{~h}^{-1}\right)$ & 2.3 & 2.0 & 2.4 & 1.9 & 1.8 & 2.1 & 2.3 & 2.4 & 1.5 & 1.5 \\
\hline SD & 0.07 & 0.13 & 0.15 & 0.11 & 0.06 & 0.04 & 0.10 & 0.10 & 0.14 & 0.12 \\
\hline$Y_{X / p h}\left(\mathrm{~g} \mathrm{~mol}_{\mathrm{ph}, \mathrm{abs}}{ }^{-1}\right)$ & 0.42 & 0.38 & 0.46 & 0.37 & 0.33 & 0.39 & 0.44 & 0.45 & 0.52 & 0.49 \\
\hline SD & 0.012 & 0.024 & 0.027 & 0.022 & 0.011 & 0.007 & 0.018 & 0.019 & 0.048 & 0.039 \\
\hline$Y_{X / p h}\left(\mathrm{~g} \mathrm{~mol}_{\mathrm{ph}, \mathrm{in}^{-1}}\right)$ & 0.41 & 0.37 & 0.45 & 0.37 & 0.32 & 0.39 & 0.44 & 0.44 & 0.51 & 0.49 \\
\hline SD & 0.012 & 0.024 & 0.027 & 0.022 & 0.011 & 0.007 & 0.018 & 0.019 & 0.048 & 0.039 \\
\hline
\end{tabular}


Table D2 Overview of light measurements and experimental results using the BF4 mutant.

\begin{tabular}{lccc}
\hline Experiment & $\mathbf{1}$ & $\mathbf{2}$ & $\mathbf{3}$ \\
\hline \hline Iph,in $\left(\mu \mathrm{mol} \mathrm{m}^{-2} \mathrm{~s}^{-1}\right)$ & 1512 & 1503 & 1503 \\
Iph,out $\left(\mu \mathrm{mol} \mathrm{m}^{-2} \mathrm{~s}^{-1}\right)$ & 25 & 50 & 100 \\
$C_{X}\left(\mathrm{~g} \mathrm{~L}^{-1}\right)$ & 4.5 & 3.6 & 2.8 \\
$\mathrm{SD}$ & 0.12 & 0.11 & 0.08 \\
$D\left(\mathrm{~d}^{-1}\right)$ & 0.6 & 0.9 & 1.2 \\
$\mathrm{SD}$ & 0.03 & 0.12 & 0.04 \\
$r_{X}\left(\mathrm{~g} \mathrm{~m}^{-2} \mathrm{~h}^{-1}\right)$ & 1.6 & 1.8 & 2.0 \\
$\mathrm{SD}$ & 0.08 & 0.25 & 0.07 \\
$Y_{x / p h}\left(\mathrm{~g} \mathrm{~mol}_{\mathrm{ph}, \mathrm{abs}}{ }^{-1}\right)$ & 0.30 & 0.34 & 0.39 \\
$\mathrm{SD}$ & 0.015 & 0.048 & 0.013 \\
$Y_{x / p h}\left(\mathrm{~g} \mathrm{~mol}_{\left.\mathrm{ph}, \mathrm{in}^{-1}\right)}\right.$ & 0.29 & 0.33 & 0.36 \\
$\mathrm{SD}$ & 0.015 & 0.046 & 0.012 \\
\hline
\end{tabular}

Table D3 Overview of light measurements and experimental results using the TLA2 mutant.

\begin{tabular}{lccc}
\hline Experiment & $\mathbf{1}$ & $\mathbf{2}$ & $\mathbf{3}$ \\
\hline \hline Iph,in $\left(\mu \mathrm{mol} \mathrm{m}^{-2} \mathrm{~s}^{-1}\right)$ & 1469 & 1479 & 850 \\
Iph,out $\left(\mu \mathrm{mol} \mathrm{m}^{-2} \mathrm{~s}^{-1}\right)$ & 52 & 100 & 52 \\
$C_{x}\left(\mathrm{~g} \mathrm{~L}^{-1}\right)$ & 2.3 & 1.8 & 1.8 \\
$\mathrm{SD}$ & 0.11 & 0.07 & 0.06 \\
$D\left(\mathrm{~d}^{-1}\right)$ & 1.2 & 1.6 & 1.14 \\
$\mathrm{SD}$ & 0.06 & 0.10 & 0.06 \\
$r_{x}\left(\mathrm{~g} \mathrm{~m}^{-2} \mathrm{~h}^{-1}\right)$ & 1.6 & 1.8 & 1.2 \\
$\mathrm{SD}$ & 0.09 & 0.10 & 0.09 \\
$Y_{x / p h}\left(\mathrm{~g} \mathrm{~mol}_{\mathrm{ph}, \mathrm{abs}}{ }^{-1}\right)$ & 0.32 & 0.35 & 0.43 \\
$\mathrm{SD}$ & 0.018 & 0.020 & 0.031 \\
$Y_{x / p h}\left(\mathrm{~g} \mathrm{~mol}_{\left.\mathrm{ph}, \mathrm{in}^{-1}\right)}\right.$ & 0.31 & 0.33 & 0.40 \\
$\mathrm{SD}$ & 0.017 & 0.019 & 0.029 \\
\hline
\end{tabular}


Table D4 Overview of light measurements and experimental results using the AS2.2 mutant.

\begin{tabular}{lcccc}
\hline Experiment & $\mathbf{1}$ & $\mathbf{2}$ & $\mathbf{3}$ & $\mathbf{4}$ \\
\hline Iph,in $\left(\mu \mathrm{mol} \mathrm{m}^{-2} \mathrm{~s}^{-1}\right)$ & 1514 & 1501 & 1500 & 855 \\
Iph,out $\left(\mu \mathrm{mol} \mathrm{m}^{-2} \mathrm{~s}^{-1}\right)$ & 25 & 50 & 100 & 50 \\
$C_{x}\left(\mathrm{~g} \mathrm{~L}^{-1}\right)$ & 2.3 & 1.9 & 1.5 & 1.5 \\
$\mathrm{SD}$ & 0.26 & 0.17 & 0.10 & 0.13 \\
$D\left(\mathrm{~d}^{-1}\right)$ & 1.0 & 1.6 & 1.8 & 1.5 \\
$\mathrm{SD}$ & 0.08 & 0.06 & 0.10 & 0.05 \\
$r_{x}\left(\mathrm{~g} \mathrm{~m}^{-2} \mathrm{~h}^{-1}\right)$ & 1.4 & 1.8 & 1.6 & 1.3 \\
$\mathrm{SD}$ & 0.17 & 0.13 & 0.08 & 0.11 \\
$Y_{x / p h}\left(\mathrm{~g} \mathrm{~mol}_{\mathrm{ph}, \mathrm{abs}}{ }^{-1}\right)$ & 0.26 & 0.34 & 0.32 & 0.46 \\
$\mathrm{SD}$ & 0.033 & 0.025 & 0.015 & 0.037 \\
$Y_{x / p h}\left(\mathrm{~g} \mathrm{~mol}_{\mathrm{ph}, \mathrm{in}^{-1}}\right)$ & 0.26 & 0.32 & 0.30 & 0.43 \\
$\mathrm{SD}$ & 0.032 & 0.025 & 0.014 & 0.035 \\
\hline
\end{tabular}

Table D5 Overview of light measurements and experimental results using the StmLR3 mutant.

\begin{tabular}{lccc}
\hline Experiment & $\mathbf{1}$ & $\mathbf{2}$ & $\mathbf{3}$ \\
\hline \hline Iph,in $\left(\mu \mathrm{mol} \mathrm{m}^{-2} \mathrm{~s}^{-1}\right)$ & 1470 & 1455 & 855 \\
Iph,out $\left(\mu \mathrm{mol} \mathrm{m}^{-2} \mathrm{~s}^{-1}\right)$ & 45 & 50 & 50 \\
$C_{x}\left(\mathrm{~g} \mathrm{~L}^{-1}\right)$ & 2.98 & 2.3 & 1.7 \\
$\mathrm{SD}$ & 0.06 & 0.08 & 0.12 \\
$D\left(\mathrm{~d}^{-1}\right)$ & 1.1 & 1.6 & 1.4 \\
$\mathrm{SD}$ & 0.02 & 0.02 & 0.05 \\
$r_{x}\left(\mathrm{~g} \mathrm{~m}^{-2} \mathrm{~h}^{-1}\right)$ & 1.9 & 2.1 & 1.4 \\
$\mathrm{SD}$ & 0.02 & 0.10 & 0.11 \\
$Y_{x / p h}\left(\mathrm{~g} \mathrm{~mol}_{\mathrm{ph}, \mathrm{abs}}{ }^{-1}\right)$ & 0.38 & 0.42 & 0.50 \\
$\mathrm{SD}$ & 0.003 & 0.019 & 0.038 \\
$Y_{x / p h}\left(\mathrm{~g} \mathrm{~mol}_{\mathrm{ph}, \mathrm{in}^{-1}}\right)$ & 0.37 & 0.41 & 0.47 \\
$\mathrm{SD}$ & 0.003 & 0.019 & 0.036 \\
\hline
\end{tabular}


Chapter 2 

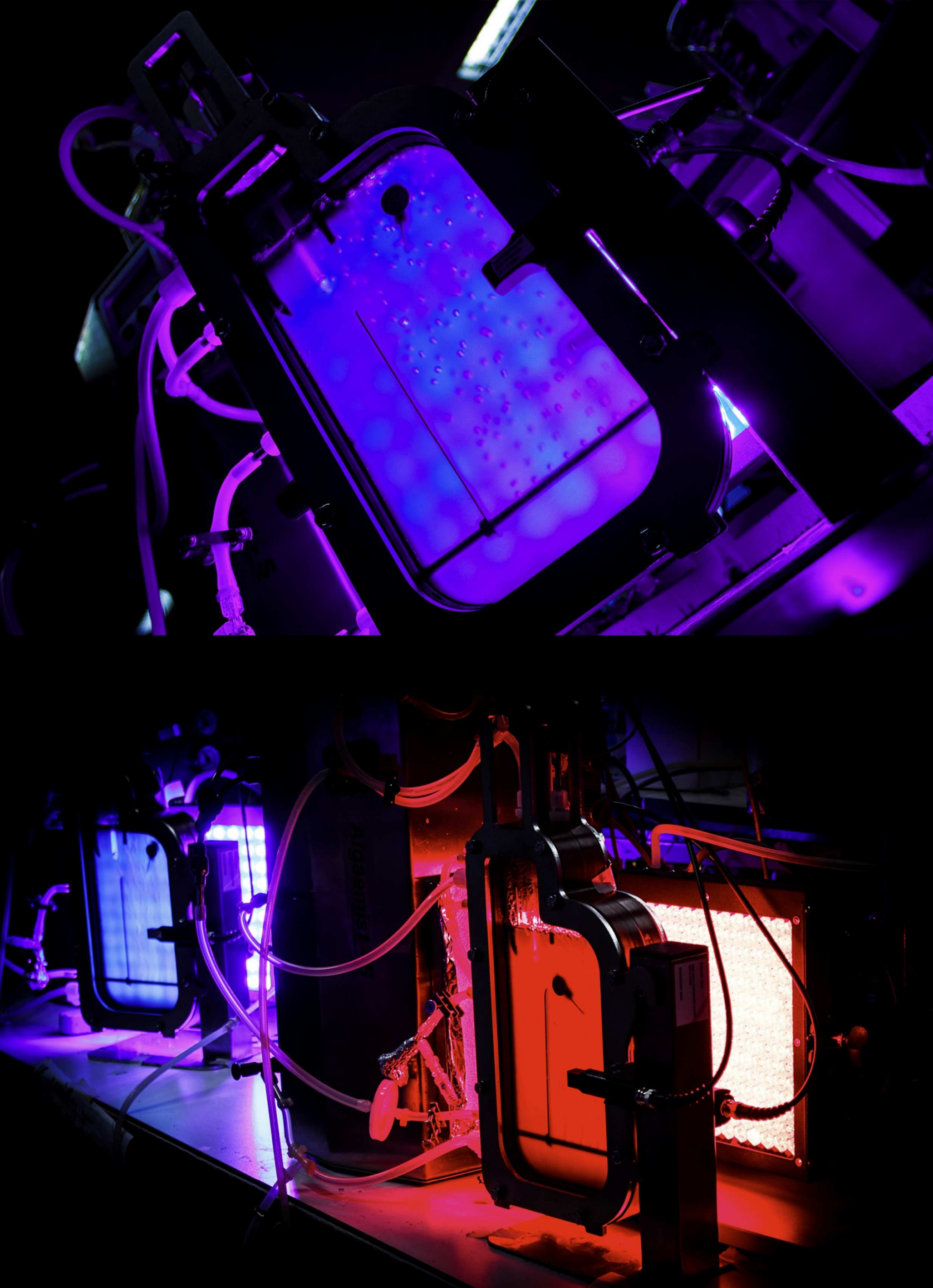


\section{Chapter 3:}

\section{Impact of light color on photobioreactor productivity}

Tim de Mooij, Guus de Vries, Christos Latsos, René H. Wijffels, Marcel Janssen 


\begin{abstract}
Microalgae perform photosynthesis at a high efficiency under low light conditions. However, under bright sunlight, it is difficult to achieve a high photosynthetic efficiency, because cells absorb more light energy than can be converted to biochemical energy. Consequently microalgae dissipate part of the absorbed light energy as heat. The objective of this study was to investigate photobioreactor productivity as a function of the biomass specific light absorption rate. A strategy to circumvent oversaturation is to exploit light with a spectral composition that minimizes light absorption. We studied productivity of Chlamydomonas reinhardtii cultivated under different colors of light. The incident light intensity was $1500 \mu \mathrm{mol}$ photons $\mathrm{m}^{-2} \mathrm{~s}^{-1}$, and cultivation took place in turbidostat controlled lab-scale panel photobioreactors. Our results demonstrate that, under mass culture conditions, productivity and biomass specific light absorption are inversely correlated. The highest productivity, measured under continuous illumination, was obtained using yellow light $\left(54 \mathrm{~g} \mathrm{~m}^{-2} \mathrm{~d}^{-1}\right)$ while blue and red light resulted in the lowest light use efficiency $\left(29 \mathrm{~g} \mathrm{~m}^{-2} \mathrm{~d}^{-1}\right)$. Presumed signs of biological interference caused by employing monochromatic light of various wavelengths are discussed. This study provides a base for different approaches to maximize productivity by lowering the biomass specific light absorption rate.
\end{abstract}




\subsection{Introduction}

Microalgae are an attractive source for biofuels and bulk chemicals due to their high photosynthetic efficiency (PE). At low light intensities, microalgae can achieve values up to $80 \%$ of the theoretical maximum $\mathrm{PE}$ of $0.125 \mathrm{~mol} \mathrm{CO}_{2}$ fixed per mol photons absorbed (Emerson et al. 1943, Björkman et al. 1987, Evans 1987, Malkin et al. 1996). However, maximum PE values, as measured under low light conditions, will never be realized in microalgae mass cultures exposed to direct sunlight. The reason is the inherent nature of light. Unlike most chemical substances, light energy cannot be dissolved in the culture medium. Therefore there will always be a steep light gradient proceeding from a high level of sunlight to virtual darkness. Because of the high incident light intensity it is practically impossible to obtain the maximum light use efficiency in microalgae mass cultures. In a high density microalgae culture, most sunlight energy is absorbed in a small volume fraction of the photobioreactor on the light-exposed side. In this volume fraction, cells are coerced to absorb more light energy than the amount that can be converted to biochemical energy by their photosynthetic machinery. This leads to oversaturation and, consequently, waste of sunlight energy through heat dissipation (Demmig-Adams et al. 2014). The result is a PE that is dramatically lower than that which can be obtained under low light conditions (Vejrazka et al. 2013).

As the photosynthetic machinery is easily oversaturated, the key to optimization is to reduce the amount of light energy absorbed per photosynthetic unit. This can be achieved by proper reactor design using the light dilution principle (Gordon 2002, Zijffers et al. 2008, Cuaresma et al. 2011). However, high material costs limit its application. Considering efficient light utilization is a bottleneck of biological nature, modifications to the light harvesting complex of microalgae would possibly be more effective (Melis 2009).

In our previous study (de Mooij et al. 2014), we evaluated the areal biomass productivity of four different antenna size mutants (Olive et al. 1981, De Vitry et al. 1988, Mussgnug et al. 2007, Bonente et al. 2011, Kirst et al. 2012) under simulated mass culture conditions. These mutants were expected to show improved productivity because of their lower pigment content compared to the wild-type thereby assuring less light absorption per cell. However, none of the studied mutants performed better than 
the wild-type, possibly due to impaired photo protection mechanisms induced by the antenna complex alterations. Another explanation is the inadvertent side effects caused by the actual process of genetic engineering resulting in reduced fitness of the strains. These genetic side effects will have to be eliminated to fully benefit from the potential of antenna size reduction by genetic engineering.

In order to demonstrate the potential of antenna size reduction on an experimental scale, light absorption can also be minimized by shifting the wavelength of the emitted light to the weakly absorbed green region. When supplying narrow-beam LED light (small peak width) at high light intensities, it is the wavelength specific absorption capacity of the algae that determines the extent of photosystem saturation and, consequently, the light use efficiency. Although there is a strong and prevalent agreement that red and blue light are optimal for algal cultivation because of the corresponding peaks in the algal absorption spectrum (Wilhelm et al. 1985, Baba et al. 2012, Kim et al. 2013, Abiusi et al. 2014, Kim et al. 2014, Schulze et al. 2014, Wang et al. 2014), the opposite could possibly be true for high density cultures. In dilute cultures, not all incoming light energy is absorbed and, therefore, light absorption is the limiting factor for maximizing productivity. On the contrary, high density cultures are characterized by the fact that all incoming light is absorbed anyway by direct or indirect control of biomass concentration via chemostat or turbidostat operation (Cuaresma et al. 2011, de Mooij et al. 2014). Therefore, since total light absorption is already guaranteed in mass cultures by applying a high biomass density, productivity is limited by the efficiency at which the absorbed light is converted to biochemical energy, and not by the efficiency of light absorption. We hypothesize that in high density mass cultures the utilization of weakly absorbed light (green-yellow) maximizes productivity while strongly absorbed light (blue and red) causes more oversaturation and is suboptimal for mass culture cultivation. Indeed, the action spectra of microalgal photosynthesis as determined by Emerson and Lewis (Emerson et al. 1943) and by Tanada (Tanada 1951) indicate that green-yellow light (500-600 nm) is used at high efficiency once it is absorbed.

A microalgal growth model (Blanken et al. 2016) was employed to estimate photobioreactor productivity as a function of light intensity and the spectral 
composition of light. The model takes into account the change of the spectral composition with increasing reactor depth because of preferential light absorption by microalgae. For example, white light becomes greener. The model allows calculation of the optimal biomass concentration $\left(C_{x}\right)$ leading to maximal productivity. For each color of light, as well as sunlight, the areal biomass productivity, the biomass specific growth rate, and the optimal biomass concentration were computed. Next to overall reactor productivity, this model provides insight into the light use efficiency at different positions in the reactor and how this depends on light color.

In this study, we aim to deliver a proof of concept that the biomass specific light absorption rate determines the volumetric biomass productivity in microalgae mass cultures. We do not consider microalgae cultivation using artificial light as a viable process for producing bulk chemicals as the associated energy costs are high whereas sunlight is at no cost and abundantly available (Blanken et al. 2013). In this study, we employ artificial light only as a tool to generate different specific light absorption rates by spectral tuning. We measured the areal biomass productivity of cultures exposed to warm white, orange-red (peak $642 \mathrm{~nm}$ ), deep-red (peak $661 \mathrm{~nm}$ ), blue (peak $458 \mathrm{~nm}$ ), and yellow light (peak $596 \mathrm{~nm}$ ). The area reflects the illuminated surface area of the photobioreactor. Cultivation took place in continuously operated bench-scale flat plate photobioreactors. For each color of light, the applied light intensity was $1500 \mu \mathrm{mol}$ photons $\mathrm{m}^{-2} \mathrm{~s}^{-1}$. The biomass concentration was controlled at a fixed level that was high enough to absorb all incoming light energy. By comparing the biomass specific light absorption rate with the measured productivity of cultures exposed to different colored lights, insight was obtained into the importance of minimizing light absorption per cell to maximize productivity.

\subsection{Materials and methods}

\subsubsection{Organisms and medium}

Chlamydomonas reinhardtii CC-1690 was obtained from the Chlamydomonas Resource Center (University of Minnesota). The algae were cultivated in a filter sterilized (pore size $0.2 \mu \mathrm{m}$ ) medium (Sueoka high salt, HS) with the following composition (in $\mathrm{g} \mathrm{L}^{-1}$ ): urea, 0.99; $\mathrm{KH}_{2} \mathrm{PO}_{4}, 0.706 ; \mathrm{K}_{2} \mathrm{HPO}_{4}, 1.465 ; \mathrm{MgSO}_{4} \cdot 7 \mathrm{H}_{2} \mathrm{O}, 0.560 ; \mathrm{CaCl}_{2} \cdot 2 \mathrm{H}_{2} \mathrm{O}, 0.114$ and 20 
$\mathrm{mL} \mathrm{L} \mathrm{L}^{-1}$ of a 100 times concentrated Hutner's trace elements solution (Hutner et al. 1950). The cultures were pre-cultivated in $250 \mathrm{~mL}$ shake flasks containing $100 \mathrm{~mL}$ of medium at pH 6.7 and at $25{ }^{\circ} \mathrm{C}$ at a light intensity of $200-300 \mu \mathrm{mol}$ photons $\mathrm{m}^{-2} \mathrm{~s}^{-1}$.

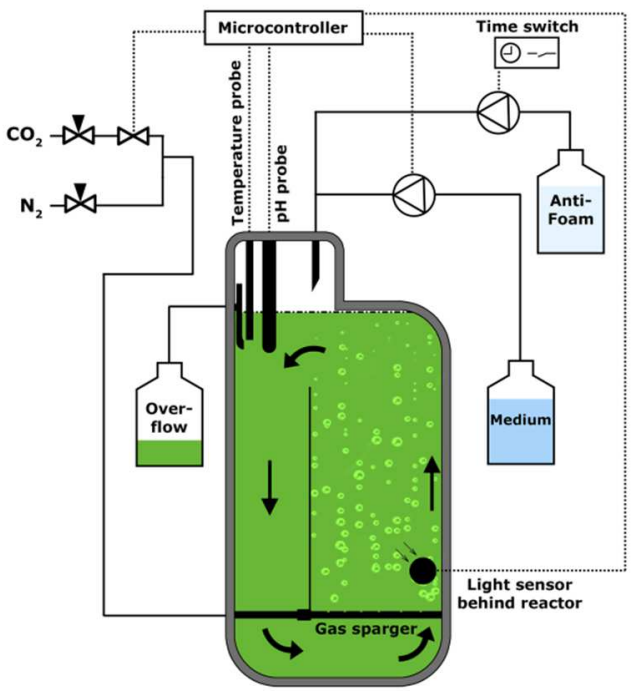

Fig. 3.1 Schematic overview of the experimental setup.

\subsubsection{Photobioreactor setup and operation}

The microalgae were continuously cultivated in flat-panel airlift photobioreactors (Algaemist, Technical Development Studio, Wageningen University, the Netherlands) with a working volume of $0.4 \mathrm{~L}$, an optical depth of $14 \mathrm{~mm}$, and an illuminated area of $0.028 \mathrm{~m}^{2}$ (See Fig. 3.1 for a schematic overview). The reactors were equipped with a black cover on the backside to prevent exposure to ambient light. Warm white light was provided by Bridgelux LED lamps (BXRA W1200, Bridgelux, USA) which are integrated in the Algaemist system. Other colors of lights were provided using $20 \times 20 \mathrm{~cm}$, SL 3500 LED panels of Photon Systems Instruments (PSI, Czech Republic). The following colors were used: blue (peak $458 \mathrm{~nm}$, spectral half-width $20 \mathrm{~nm}$ ); orange-red (642 nm; $20 \mathrm{~nm}$ ); deep red (661 nm; $20 \mathrm{~nm}$ ); and yellow (596 nm; $60 \mathrm{~nm}$ ). The yellow light source was equipped with an optical low-pass filter $(630 \mathrm{~nm})$ to cut of red light. Unless explicitly stated otherwise, all cultures grown in yellow light described in this paper were supplemented with $\pm 50 \mu \mathrm{mol}$ photons $\mathrm{m}^{-2} \mathrm{~s}^{-1}$ of blue light. The rationale behind this procedure is clarified in the results section of this paper. In Fig. 3.2 the emission spectra of all light sources are shown and these are supplemented with the solar light spectrum and the wavelength specific absorption cross section of Chlamydomonas reinhardtii. 
Please refer to Appendix D for a light intensity distribution across the illuminated reactor surface, which is provided for each light source. Reactor temperature was maintained at $25^{\circ} \mathrm{C}$, and the pH was kept at $6.7( \pm 0.1)$ by means of on-demand $\mathrm{CO}_{2}$ supply. The medium that was fed to the reactor had a $\mathrm{pH}$ of 7.0 and to maintain the setpoint of 6.7 in the reactor, $\mathrm{CO}_{2}$ supply rate was such that both $\mathrm{CO}_{2}$ and $\mathrm{HCO}_{3}$ - were present at concentrations of at least a magnitude higher than the saturation constant of Rubisco for $\mathrm{CO}_{2}$ and $\mathrm{HCO}_{3}$. The reactors were operated in turbidostat mode to ensure a constant light regime; a light sensor measures the transmission through the reactor and if light transmission was below the setpoint, the culture was automatically diluted with fresh medium employing a peristaltic pump. Further details of the photobioreactor setup and its operation are provided in de Mooij et al. (2014), with the exception that the gas stream of di-nitrogen was, at all times, $200 \mathrm{~mL} \mathrm{~min}^{-1}( \pm 20)$.

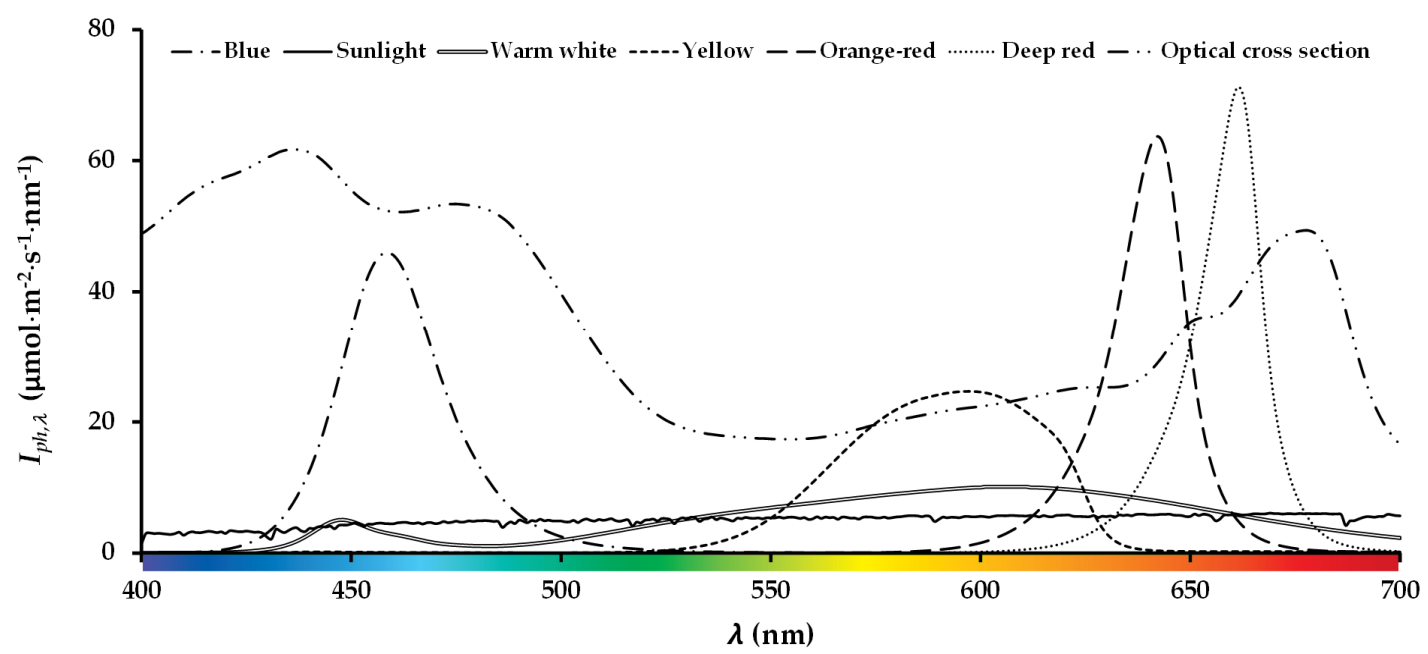

Fig. 3.2 Emission spectra of the PSI LED panels (blue, orange-red, deep red and yellow) and the Algaemist Bridgelux LED panel (warm white). The curves illustrate a photon flux density of $1500 \mu \mathrm{mol}$ photons $\mathrm{m}^{-2} \mathrm{~s}^{-1}$ when integrated across the entire PAR range (400-700 nm). This is the light intensity employed in the experiments. In addition, the solar light spectrum and the absorption cross section $\left(a_{x, \lambda}\right)$ of Chlamydomonas reinhardtii (unitless) are plotted. Numerical values for $a_{x, \lambda}$ and all emission spectra can be found online in the supplementary material (de Mooij et al. 2016). The ASTM G173-03 standard solar spectrum was obtained from the website of the National Renewable Energy Laboratory (NREL, USA). 


\subsubsection{Analyses}

\section{Biomass dry weight concentration}

To determine the biomass dry weight content, the culture broth was passed through glass fiber filters as described by Kliphuis et al. (Kliphuis et al. 2012) and, subsequently, the mass difference between the dried empty filters and the dried filters with microalgae was recorded. All measurements on an individual sample were performed in triplicate.

\section{Absorption cross section}

Light absorption was measured in a double beam spectrophotometer (UV-2600, Shimadzu, Japan) equipped with an integrating sphere (ISR-2600). A reactor sample was transferred to a cuvette (100.099-OS, Hellma, Germany) with a short light path of $2 \mathrm{~mm}$. The same reactor sample was analysed for its dry weight content. This allowed normalisation of the absorption cross section, resulting in a biomass specific absorption cross section. Additional details of this protocol have been described by de Mooij et al. (de Mooij et al. 2014). Only samples from the cultures grown under yellow and warm white light were diluted with medium (1:1) because of the higher biomass density. All other samples were not diluted.

\section{Maximum photosystem II quantum yield}

The maximum photosystem II (PSII) quantum yield $\left(F_{v} / F_{m}\right)$ was measured from samples withdrawn directly from the reactor. Samples were diluted to obtain a biomass density of approximately $25 \mathrm{mg} \mathrm{L}^{-1}$. Following dark adaptation for ten minutes, fluorescence of the microalgae samples was measured with a technique based on pulse amplitude modulated (PAM) fluorometry (AquaPen-C AP-C 100, PSI, Czech Republic). According to the manufacturer's manual, the actinic light intensity was $270 \mu \mathrm{mol}$ photons $\mathrm{m}^{-2} \mathrm{~s}^{-1}$; the saturating light intensity was $2430 \mu \mathrm{mol}$ photons $\mathrm{m}^{-2} \mathrm{~s}^{-1}$; and the measuring light intensity was $0.03 \mu \mathrm{mol}$ photons $\mathrm{m}^{-2} \mathrm{~s}^{-1}$. The excitation light wavelength was $620 \mathrm{~nm}$. $F_{v} / F_{m}$ is the ratio of variable fluorescence to maximal fluorescence (eq. 1). In this equation, $F_{o}$ is the dark-adapted minimum level of fluorescence. $F_{m}$ is the maximum level of fluorescence, measured after a short light pulse of high intensity. $F_{v}$ (variable fluorescence) is defined as the difference between $F_{m}$ and $F_{o}$, and represents the difference between fluorescence intensities with closed and open reaction centers.

$$
F_{v} / F_{m}=\left(F_{m}-F_{0}\right) / F_{m}
$$




\subsection{Results}

\subsubsection{Model estimation of biomass productivity and biomass yield on light for different colors of light}

Photobioreactor productivity was estimated employing a microalgae growth model. The model predicts photosynthetic rates at every location in the reactor based on the local light intensity. The light intensity is calculated for each wavelength at every point in the reactor to account for preferential light absorption by microalgae and the resulting change in spectrum composition. A description of the model and a list of the model parameters used are located in Appendix A. The following model calculations are based on parameters used in the experiments performed: an ingoing light intensity of 1500 $\mu \mathrm{mol}$ photons $\mathrm{m}^{-2} \mathrm{~s}^{-1}$, a reactor depth of $14 \mathrm{~mm}$, and the absorption cross section of a continuous mass culture grown under warm white light in turbidostat mode (see Fig. 3.2). This mass culture was characterized by complete absorption of the incident light and with an outgoing light intensity of $10 \mu \mathrm{mol}$ photons $\mathrm{m}^{-2} \mathrm{~s}^{-1}$. These conditions were chosen to maximize reactor productivity as for $C$. reinhardtii, $10 \mu \mathrm{mol}$ photons $\mathrm{m}^{-2} \mathrm{~s}^{-1}$ was found to be the photosynthetic compensation point (Takache et al. 2010, Vejrazka et al. 2013), where the net photosynthesis rate is equal to zero. At higher biomass concentrations, dark zones are created where cell maintenance is a dominant process, which reduces the reactor productivity. At lower biomass concentrations, light passes the culture without being absorbed and without contributing to the overall productivity. Therefore, in this study light color was studied at biomass concentrations that were optimized for each light color. Running the cultivations at the same fixed biomass concentration would not be a fair comparison as it does not allow to use the full potential of each color of light. Biomass concentration optimization is essential in a mass culture setup to maximize biomass productivity.

Using the model, we estimated the optimal biomass concentration $\left(C_{x}\right)$ for each color of light to maximize the areal productivity, assuming the bioreactor is operated at a constant biomass density (turbidostat) and constant light intensity. As can be seen in Fig. 3.3, strongly absorbed light in colors such as blue and deep red result in low biomass concentrations (1.0 $\mathrm{g} \mathrm{L}^{-1}$ and $1.3 \mathrm{~g} \mathrm{~L}^{-1}$, respectively) while a weakly absorbed light in a color such as yellow gives a biomass concentration of $2.8 \mathrm{~g} \mathrm{~L}^{-1}$. By combining the local light absorption rate with the estimated biomass concentrations for all light 
sources, we calculated the biomass specific light absorption $\left(q_{p h}, \operatorname{mol}_{\mathrm{ph}} \operatorname{mol}_{\mathrm{x}}{ }^{-1} \mathrm{~s}^{-1}\right)$ at each position inside the photobioreactor (Fig. 3.4A). Under the described conditions, blue light results in the highest $q_{p h}$ while the cultures grown under yellow light absorb the least light energy per unit of biomass. Fig. 3.4B illustrates the local specific growth rate $(\mu)$ as a function of the local light intensity in the reactor. Each light intensity corresponds to a certain location in the reactor. A culture exposed to blue light grows at $\mu_{\max }$ if the light intensity is higher than approximately $100 \mu \mathrm{mol}$ photons $\mathrm{m}^{-2} \mathrm{~s}^{-1}$ while a culture exposed to yellow light requires about $500 \mu \mathrm{mol}$ photons $\mathrm{m}^{-2} \mathrm{~s}^{-1}$ to support maximum growth. Although it shows the high sensitivity for blue light, this does not imply that the reactor productivity of a mass culture will be higher under blue light. The reason is that when grown under blue light, only low biomass concentrations can be supported and that the light use efficiency is low which limits the volumetric productivity. The spatially averaged $\mu$ values can be found in Fig. 3.3. A table with all the estimated model values can be found in Appendix B.

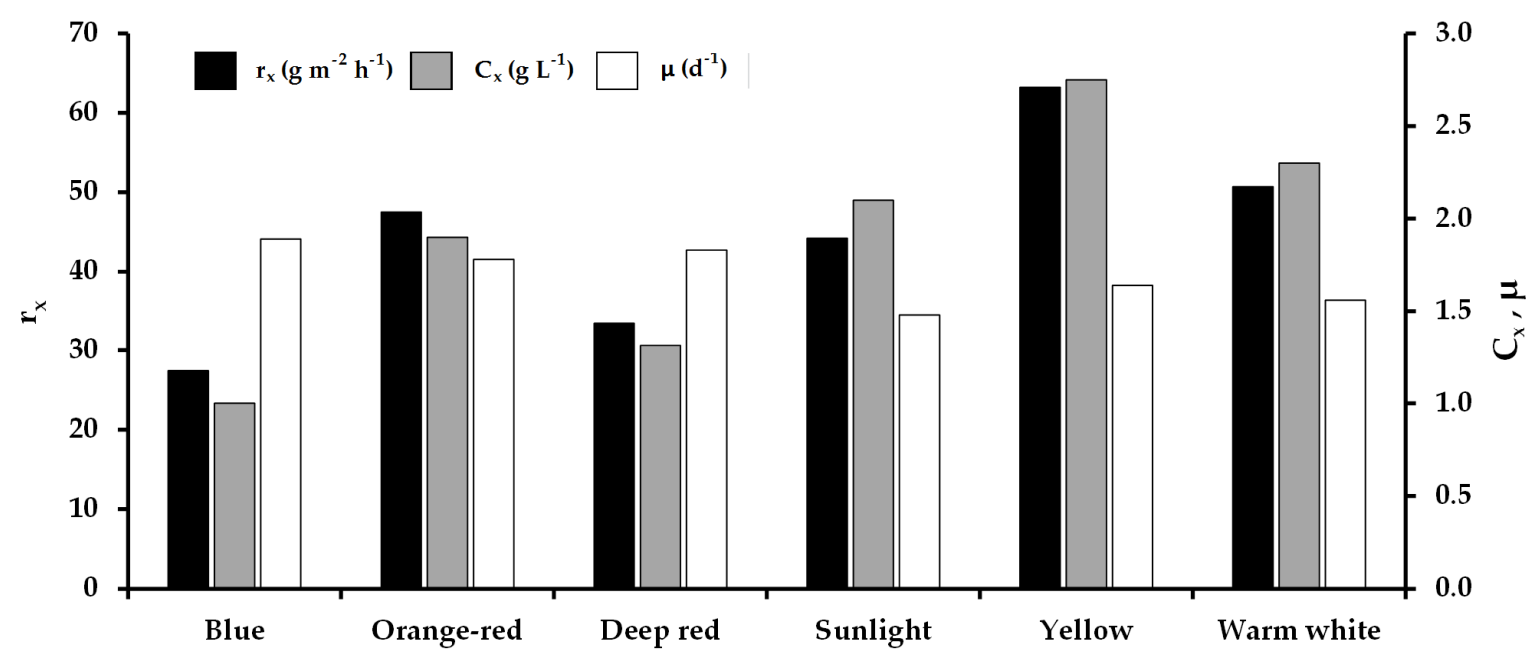

Fig. 3.3 Model estimation of areal biomass productivity $\left(r_{x}\right)$, biomass concentration $\left(C_{x}\right)$, and biomass specific growth rate $(\mu)$ at $I_{p h, i n}=1500 \mu$ mol photons $m^{-2} s^{-1}$ for different light colors.

Fig. 3.4C depicts the local biomass yield on light energy $\left(\operatorname{mol}_{\mathrm{x}} \mathrm{mol}_{\mathrm{ph}}{ }^{-1}\right)$ as a function of reactor depth. In general, it can be observed that, in a mass culture, weakly absorbed light results in higher yields than strongly absorbed light. In the deeper, darker part of the reactor, the biomass yield on light energy $\left(Y_{x / p h}\right)$ decreases for all colors of light as cell maintenance becomes a significant factor relative to the photosynthetic activity. To maximize productivity, the biomass concentration was chosen in such a way, that at the 
back of the reactor the local biomass yield on light is zero. This is at the photosynthetic compensation point. Stated differently, at every position in the reactor there is a positive contribution to the reactor productivity.

The biomass productivity expressed per unit of illuminated surface area $\left(r_{x}\right)$ is presented in Fig. 3.3. The highest productivity $\left(63 \mathrm{~g} \mathrm{~m}^{-2} \mathrm{~d}^{-1}\right)$ is predicted for cultures exposed to yellow light while the strongly absorbed blue light is expected to result in a productivity of $27 \mathrm{~g} \mathrm{~m}^{-2} \mathrm{~d}^{-1}$. Warm white light, whose spectrum contains a significant fraction of weakly absorbed light (e.g., green and yellow), results in productivity as high as $51 \mathrm{~g} \mathrm{~m}^{-2} \mathrm{~d}^{-1}$. Deep red light is estimated to result in lower productivity than orangered light. This is explained by the fact that the deep red light spectrum is overlapping the chlorophyll a absorption peak (see Fig. 3.2) while the orange-red peak is located in a less absorbing region of the algae absorption spectrum.

The light spectrum changes with increasing reactor depth because of the preferential absorption of blue and red light by green microalgae. The light becomes greener as the red and blue fractions are rapidly absorbed. As a consequence, warm white LED light and sunlight are quickly converted into green light with increasing culture depth. As is evident from Fig. 3.4A and Fig. 3.4C, the color of light influences the local $q_{p h}$ and $Y_{x / p h}$ primarily in the first $2 \mathrm{~mm}$ of the culture. In high light conditions at the surface of the reactor, the highest $Y_{x / p h}$ is observed employing yellow light. However, at a depth $\geq 2$ $\mathrm{mm}$, higher yields can be obtained with warm white light and sunlight. Considering that $53 \%$ of the incoming light energy is absorbed within the first $2 \mathrm{~mm}$, the photosynthetic efficiency in this surface layer has a dominant effect on reactor productivity. 

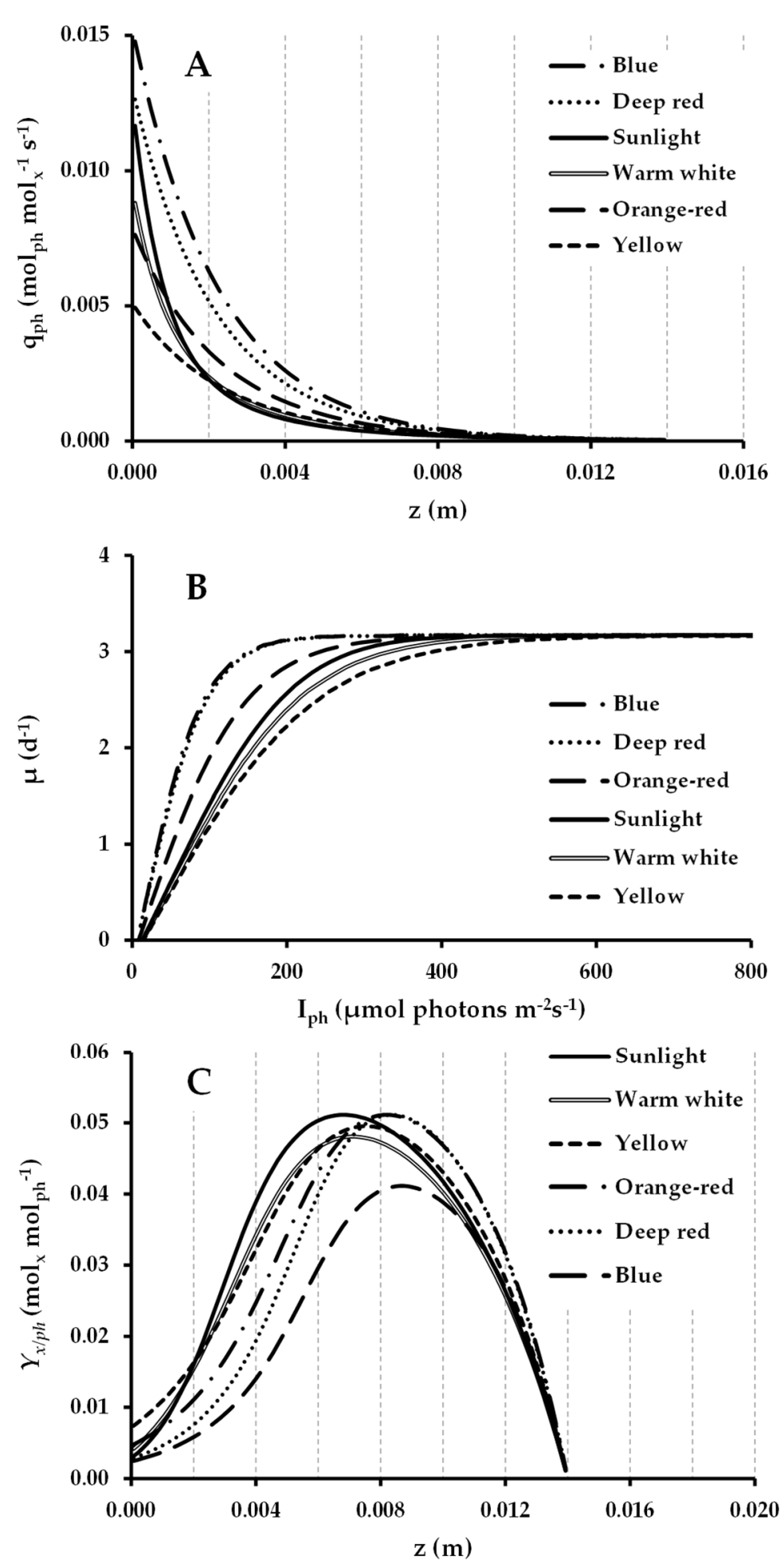

Fig. 3.4 (A) Model estimation of the biomass specific light absorption rate $\left(q_{p h}\right)$ as a function of location $z$ in the reactor. (B) Estimation of the local biomass specific growth rate $(\mu)$ as a function of the local light intensity. The spatially averaged $\mu$ can be found in Fig. 3.3. At light intensities exceeding \pm 500 umol photons $\mathrm{m}^{-2} \mathrm{~s}^{-1}$, all colors of light generate photosystem saturation, resulting in maximum growth rate. Blue and deep red light begin to saturate at \pm $100 \mu \mathrm{mol}$ photons $\mathrm{m}^{-2} \mathrm{~s}^{-1}$. (C) Biomass yield on light energy $\left(Y_{x / p h}\right)$ as a function of location $z$ in the reactor. Of major interest is the reactor volume near the light exposed reactor surface (depth: 0 $0.002 \mathrm{~m}$ ) as most light energy is absorbed in this region.

\subsubsection{Experimental areal biomass productivity for different colors of light}

Based on the model predictions, a large difference in productivity can be expected between weakly and strongly absorbed light colors. Except for sunlight, we performed reactor experiments with all of the colors of lights mentioned. Areal biomass 
productivity was measured at an ingoing light intensity $\left(I_{p h, i n}\right)$ of $1500 \mu \mathrm{mol}$ photons $\mathrm{m}^{-2}$ $\mathrm{s}^{-1}$. The outgoing light intensity $\left(I_{p h, o u t}\right)$ was maintained at $10 \mu \mathrm{mol}$ photons $\mathrm{m}^{-2} \mathrm{~s}^{-1}$ by turbidostat control. The cultures exposed to yellow light were subjected to $1450 \mu \mathrm{mol}$ photons $\mathrm{m}^{-2} \mathrm{~s}^{-1}$ and supplemented with $50 \mu \mathrm{mol}$ photons $\mathrm{m}^{-2} \mathrm{~s}^{-1}$ of blue light, as will be discussed in detail later. In Fig. 3.5, the areal biomass productivity, biomass concentration, and the dilution rate are presented. The highest productivity was obtained employing yellow light $\left(54 \mathrm{~g} \mathrm{~m}^{-2} \mathrm{~d}^{-1} \pm 5\right)$. A slightly lower value was found for warm white light (50 $\mathrm{g} \mathrm{m}^{-2} \mathrm{~d}^{-1} \pm 2$ ). Cultures exposed to blue, orange-red, and deep red all yielded a productivity of approximately $29 \mathrm{~g} \mathrm{~m}^{-2} \mathrm{~d}^{-1}$. For the exact values of light intensity and obtained experimental data of each experiment, please refer to Appendix C.

The highest biomass concentration was measured for cultures exposed to yellow light (2.96 $\left.\mathrm{g} \mathrm{L}^{-1} \pm 0.12\right)$ and the lowest for cultures grown under blue light $\left(0.92 \mathrm{~g} \mathrm{~L}^{-1} \pm 0.05\right)$. Since all cultures were turbidostat controlled and were as such, forced to absorb 1490 $\mu \mathrm{mol}$ photons $\mathrm{m}^{-2} \mathrm{~s}^{-1}$, the biomass concentration presented in Fig. 3.5B inherently demonstrates the ability of the algal biomass to absorb light of different colors. A low biomass concentration corresponds to a relatively high biomass specific light absorption rate which was accompanied by a high rate of energy dissipation.

In our experiments, the specific growth rate $\mu$ equals the reactor dilution rate $D$, as can be deduced from the biomass balance over the photobioreactor (de Mooij et al. 2014). As expected, the low biomass concentration in cultures grown under blue light is accompanied by a high dilution rate $\left(2.2 \mathrm{~d}^{-1} \pm 0.04\right)$ because cells cultivated under blue light will be light saturated at relatively low light intensities. Compared to other light colors, the light intensity will be high enough to saturate the cells in a larger volume fraction of the reactor. The result is a higher spatially averaged biomass specific growth rate. However, since volumetric productivity is the product of biomass concentration and dilution rate, the low biomass concentration limits productivity. It is remarkable that, even though the culture exposed to orange-red exhibited a lower biomass concentration than cultures grown under warm white, which indicated a higher biomass specific light absorption, this lower biomass concentration was accompanied by a lower dilution rate. 
The maximum $F_{v} / F_{m}$ value of dark adapted samples (see Fig. 3.5D) withdrawn from the reactor represents photosystem II quantum efficiency and is an indicator of photoinhibition or down-regulation of photosystem II activity (Demmig et al. 1987, Young et al. 2003, Logan et al. 2007). The highest values were obtained for the cultures exposed to blue $(0.63 \pm 0.02)$ and white $(0.64 \pm 0.05)$ light. The lowest $F_{v} / F_{m}$ value was obtained for the cultures exposed to yellow light $(0.50 \pm 0.04)$. Cultures exposed to orange-red light also demonstrated reduced values $(0.53 \pm 0.06)$, indicating that photosystems did not function at full capacity.
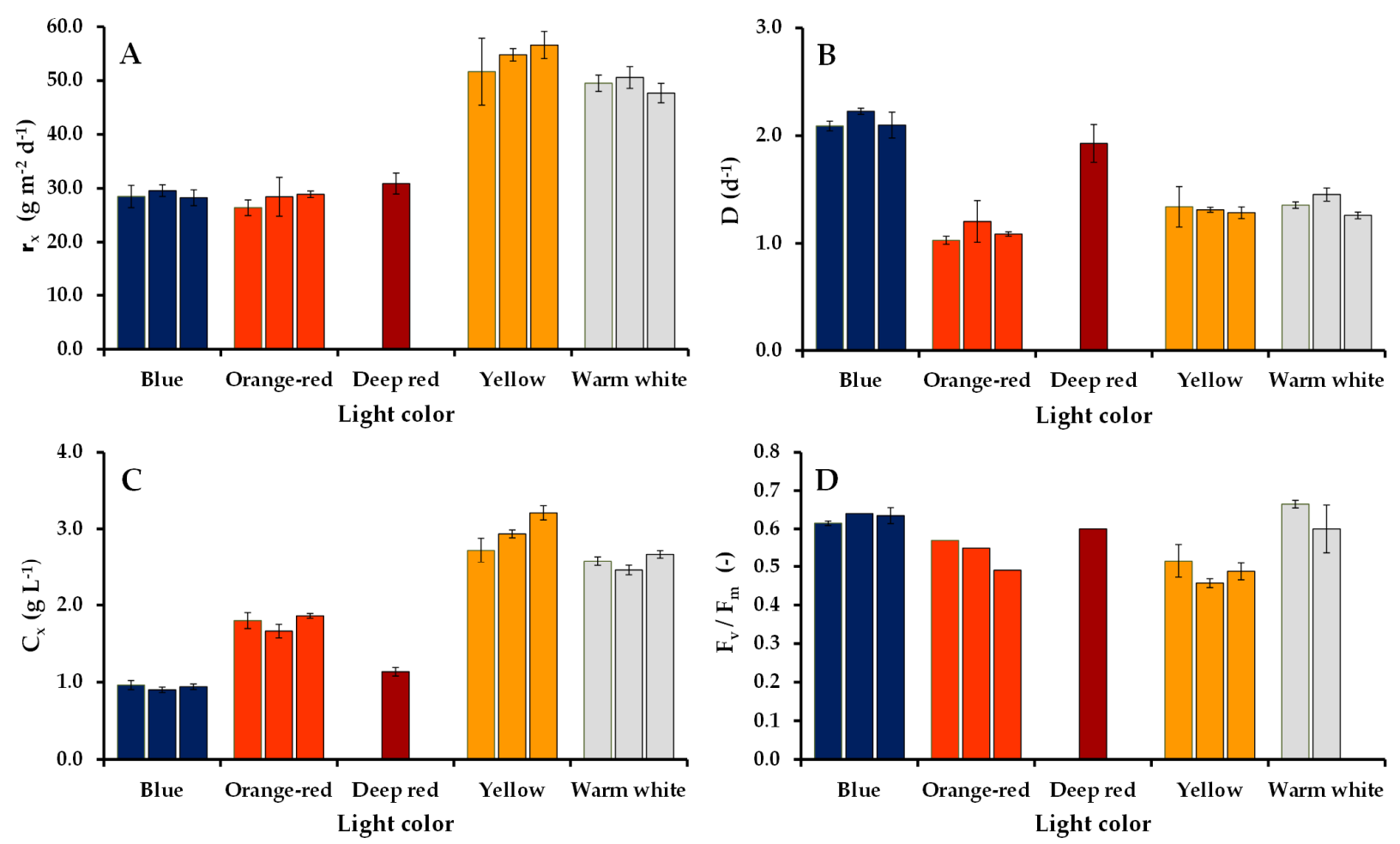

Fig. 3.5 (A) Areal biomass productivity $\left(r_{x}\right)$. (B) dilution rate $(D=\mu)$. (C) biomass concentration $\left(C_{x}\right)$. (D) dark adapted maximum $F_{v} / F_{m}$. The cultures were continuously operated in turbidostat mode at $I_{p h, i n}=$ $1500 \mu \mathrm{mol}$ photons $\mathrm{m}^{-2} \mathrm{~s}^{-1}, I_{\text {ph,out }}=10 \mu \mathrm{mol}$ photons $\mathrm{m}^{-2} \mathrm{~s}^{-1}$. Each bar in $(A),(B)$, and $(C)$ represents the average $\pm S D$ of $n \geq 4$ data points that were measured on a daily basis within one reactor experiment.

Cultivation under both orange-red and deep-red light was difficult. Several experiments at an $I_{p h, i n}$ of $1500 \mu \mathrm{mol}$ photons $\mathrm{m}^{-2} \mathrm{~s}^{-1}$ failed as no stable growth could be obtained. In most cases, there was biomass growth for a few days, after which growth suddenly ceased completely and was accompanied by cell agglomeration. In some cultivations, productivity fluctuated considerably from day to day. Applying orange-red illumination, three out of six experiments were successful, which means that stable, day to day productivity values were obtained for at least six days. Applying deep red light, only one 
experiment out of five was successful. Assuming that the high light intensity did not allow unconstrained growth of the algae when applying deep red light, two additional experiments were performed at an incident light intensity of $850 \mu \mathrm{mol}$ photons $\mathrm{m}^{-2} \mathrm{~s}^{-1}$ and an outgoing light intensity of $10 \mu \mathrm{mol}$ photons $\mathrm{m}^{-2} \mathrm{~s}^{-1}$. As depicted in Fig. 3.6, cultures grown under deep red had a lower biomass concentration and, therefore, a higher biomass specific light absorption rate compared to those grown under warm white light. The dilution rate, however, was not higher compared to cultures grown under warm white light and, therefore, the productivity was also lower (18 vs $36 \mathrm{~g} \mathrm{~m}^{-2} \mathrm{~d}^{-}$ ${ }^{1}$ for warm white). Otherwise stated, at $850 \mu \mathrm{mol}$ photons $\mathrm{m}^{-2} \mathrm{~s}^{-1}$, the light use efficiency of deep-red light was also lower than for warm white light. Maximum $F_{v} / F_{m}$ values were low $(0.50 \pm 0.03)$ for the deep red culture. The culture grown under white light exhibited a $F_{v} / F_{m}$ value of 0.61
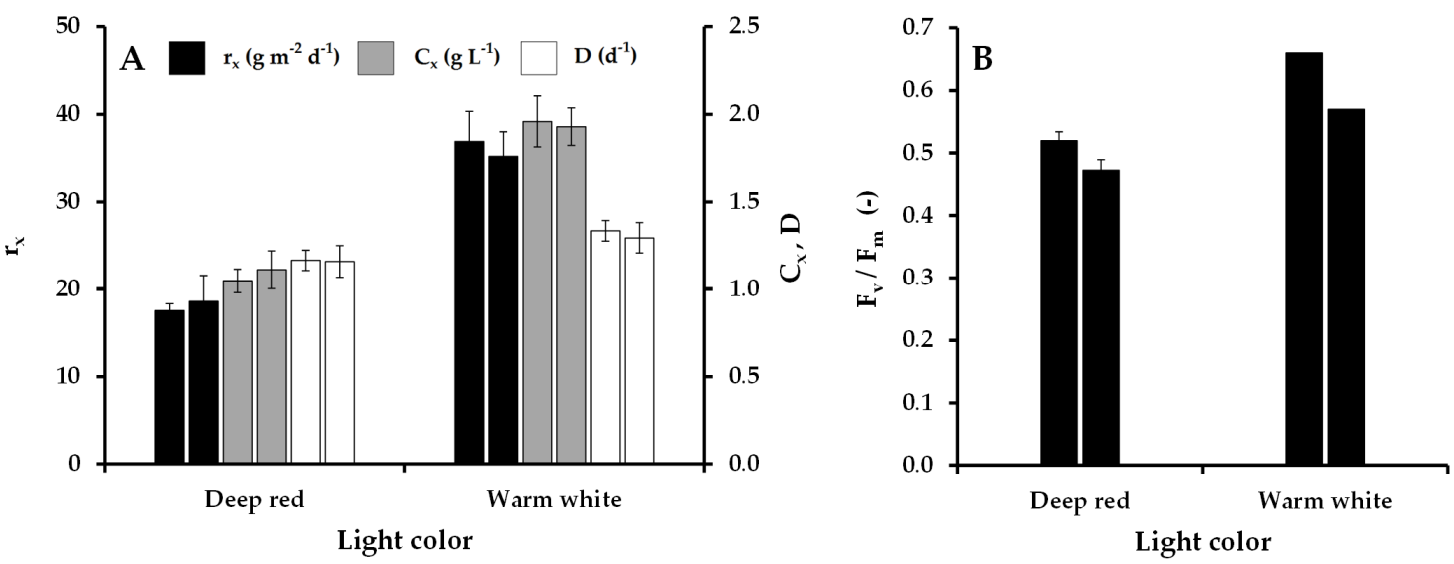

Fig. 3.6 (A) Areal biomass productivity $\left(r_{x}\right)$, biomass concentration $\left(C_{x}\right)$, dilution rate $(D=\mu)$. $(B)$ dark adapted maximum $F_{v} / F_{m}$. Reactors were operated at $I_{p h, i n}=850 \mu \mathrm{mol}$ photons $\mathrm{m}^{-2} \mathrm{~s}^{-1}, I_{p h, o u t}=10 \mu \mathrm{mol}$ photons $m^{-2} s^{-1}$. Each bar in (A) represents the average $\pm S D$ of $n \geq 5$ data points that were measured on a daily basis within one reactor experiment. The white light data was obtained from de Mooij et al. (2014).

\subsubsection{Absorption cross section under different light colors}

Fig. 3.7 shows the measured light absorption spectra of cultures grown under different light colors. In the continuously operated turbidostat cultures with ingoing light intensities as high as $1500 \mu \mathrm{mol}$ photons $\mathrm{m}^{-2} \mathrm{~s}^{-1}$, the absorption cross section of the microalgae did not markedly change as a function of light color. Up to seven measurements were performed per culture and the experimental variation within these measurements was higher than the variation between the different cultures. 


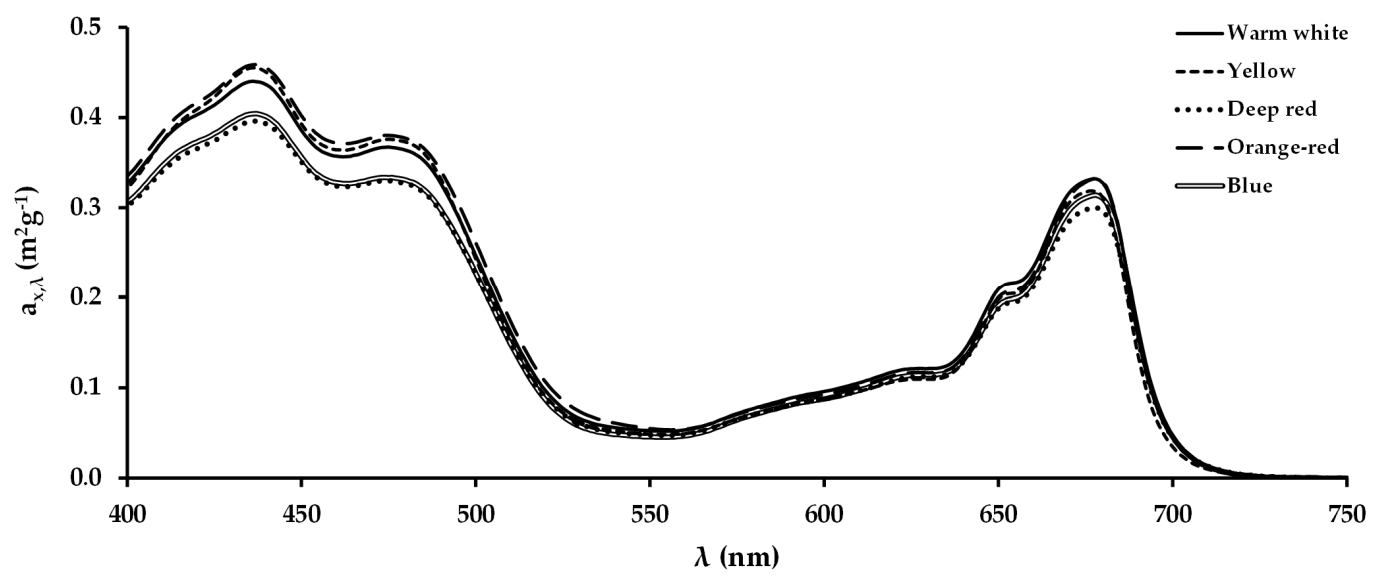

Fig. 3.7 Absorption cross section $\left(a_{x, \lambda}\right)$ of Chlamydomonas reinhardtii exposed to different colors of light. The optical cross section has been normalized to the measured biomass concentration in the reactor. Lines represent the average of at least seven measurements from three reactor experiments. For the deep red culture, the line represents the average of three measurements within a single reactor experiment.

\subsubsection{The effect of blue light supplementation to monochromatic yellow light}

We began our experiments exploiting a single yellow light source. Productivity was far below what was estimated. The cultures were unstable as productivity and biomass concentration fluctuated from day to day. In addition, maximum $F_{v} / F_{m}$ values were low, indicating a low PSII quantum efficiency. Pigment content was also considerably lower than measured for all other light colors. For this reason, the yellow light was supplemented with a moderate quantity $\left( \pm 50 \mu \mathrm{mol}\right.$ photons $\left.\mathrm{m}^{-2} \mathrm{~s}^{-1}\right)$ of blue light. The total light intensity thus was $1500 \mu \mathrm{mol}$ photons $\mathrm{m}^{-2} \mathrm{~s}^{-1}$. Unless explicitly stated otherwise (see Fig. 3.8), all cultures grown in yellow light described in this paper were supplemented with $\pm 50 \mu \mathrm{mol}$ photons $\mathrm{m}^{-2} \mathrm{~s}^{-1}$ of blue light. By applying blue light supplementation, the volumetric productivity increased from $37 \mathrm{~g} \mathrm{~m}^{-2} \mathrm{~d}^{-1} \pm 11$ to $52 \mathrm{~g}$ $\mathrm{m}^{-2} \mathrm{~d}^{-1} \pm 8$ (Fig. 3.8A), and cultivation was more stable. Furthermore, the maximum $F_{v} / F_{m}$ value was clearly higher $(0.49 \pm 0.05$ vs $0.34 \pm 0.06)$, indicating improved functioning of photosystem II (Fig. 3.8B). The absorption cross section, depicted in Fig. $3.8 \mathrm{C}$, was demonstrated to be higher in the case of blue light supplementation. The ratio between absorption by carotenoids and chlorophyll a was comparable for both situations. 

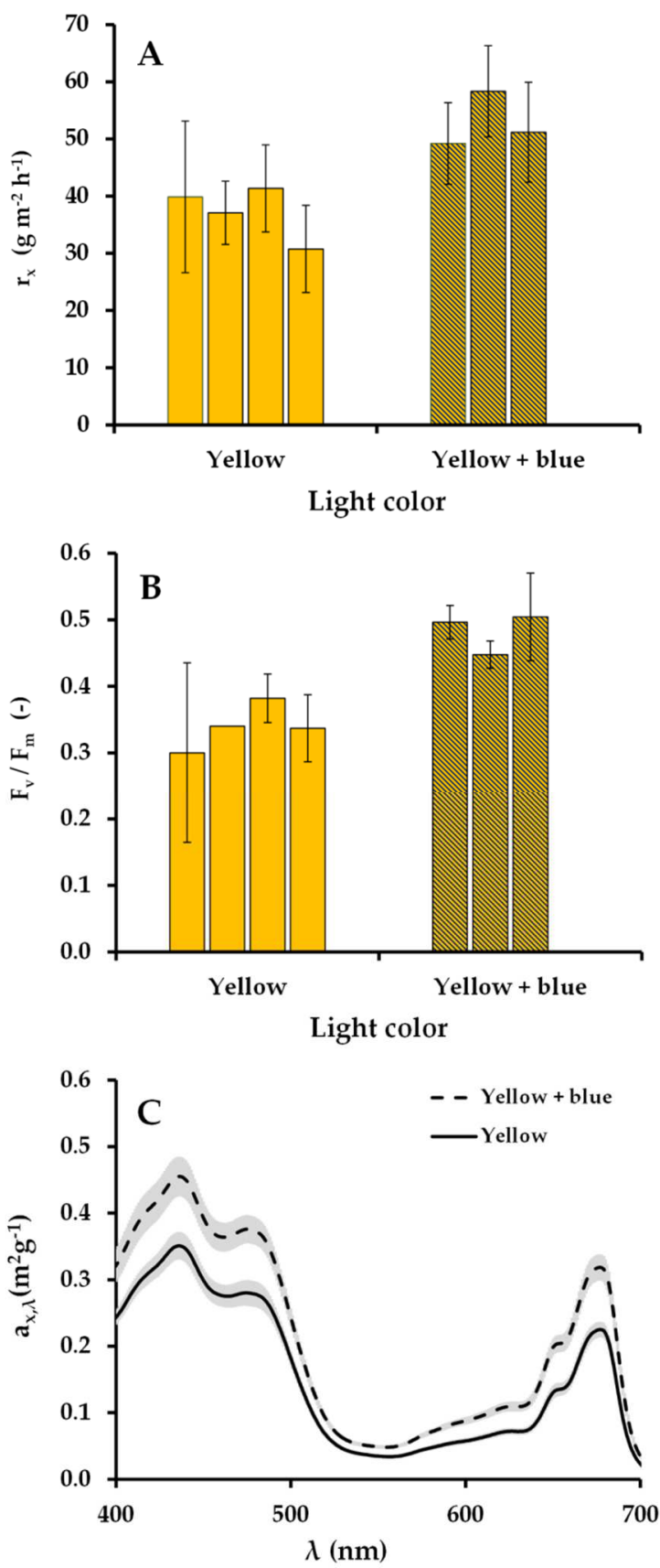

Fig. 3.8 (A) Areal biomass productivity $\left(r_{x}\right)$. (B) Dark adapted maximum $F_{v} / F_{m}$. (C) Absorption cross section $\left(a_{x, \lambda}\right)$. The cultures were exposed to yellow light and yellow light supplemented with \pm $50 \mu \mathrm{mol}$ photons $\mathrm{m}^{-2} \mathrm{~s}^{-1}$ of blue light. Each bar in (A) represents the average $\pm S D$ of $n \geq 4$ data points that were measured on a daily basis within one reactor experiment. The lines in $(C)$ represent the average $\pm S D$ of at least ten measurements within three reactor experiments.

\subsection{Discussion}

Microalgae photosynthesis is inefficient at high light intensities. Not considering photobioreactor design, two approaches can be distinguished to increase the photosynthetic efficiency: genetic engineering of the microalgae or spectrally tailoring the light source via light engineering. Our previous study showed that the current generation of Chlamydomonas antenna size mutants is not able to outperform the productivity of the wild-type strain under mass culture conditions (de Mooij et al. 2014). 
To provide a more solid foundation for the hypothesis that biomass productivity is a function of the amount of light absorbed per cell, in this work, we shifted the emission of artificial illumination to both the low and high absorption region of the spectrum by selecting four different colors of light.

Our model successfully predicted the biomass productivity for different colors of light. The biomass concentration could be accurately estimated as in a turbidostat controlled culture this is a function of the incident light intensity, the outgoing light intensity, and the absorption cross section $\left(a_{x, \lambda}\right)$ of the cells. Calculation of the dilution rate, and the areal productivity is more challenging since there are many factors that influence the light use efficiency. The model assumes that good mixing prevents severe photodamage and therefore, photoinhibition is not considered. At very high light intensities, this assumption might not be valid, rendering the model prediction overly optimistic. Our model assumes that the microalgae suspension is exposed to a homogeneous light intensity distribution. In reality there can be substantial differences between for example the middle of the light exposed surface and the relatively dark corners. Depending on the distribution, this may lead to under- or overestimation of biomass productivity. Please refer to Appendix D to see the light intensity distribution of the light sources that were employed in our experiments.

In accordance with our model predictions, cultures exposed to yellow light resulted in the highest areal productivity $\left(54 \mathrm{~g} \mathrm{~m}^{-2} \mathrm{~d}^{-1}\right)$ closely followed by cultures grown under warm white light $\left(50 \mathrm{~g} \mathrm{~m}^{-2} \mathrm{~d}^{-1}\right)$. The three strongly absorbed colors (blue, orange-red, and deep red) resulted in areal productivities that were almost half of the areal productivity measured for yellow light. Cultures were difficult to grow under red light and this affected the productivity. However, our substantial number of successful reactor experiments with different colors of light confirms our model-based expectation that, under mass culture conditions, productivity is inversely correlated with biomass specific light absorption.

Analogous to our results, Kubin et al. (Kubín et al. 1983) also showed that maximal productivity with Chlorella vulgaris was obtained exploiting weakly absorbed green light. They also measured productivity values for blue light as being half of that for green and white light. Mattos et al. performed short term oxygen evolution experiments and 
conclude that weakly absorbed colors of light such as green results in a higher photosynthetic efficiency for high density cultures (Mattos et al. 2015). In these experiments the cells were not allowed to acclimate to the different colors of light and the applied light regime during the measurements and therefore these conditions do not simulate mass culture conditions. Instead of replacing blue and red light by green light, they suggest that green light should be supplemented to strongly absorbed colors of light.

The amount of nitrogen source present in the cultivation medium supports biomass concentrations up to $4.5 \mathrm{~g} \mathrm{~L}^{-1}$. To ensure that nitrogen limitation did not occur, we increased the urea content for the cultures exposed to yellow light. There was no measurable effect of the urea supplementation and, therefore, we conclude that the medium was indeed sufficient for unconstrained growth.

No substantial difference in absorption cross section of the microalgae was observed after cultivating them under different light colors. Apparently, under mass culture conditions and irrespective of the color of light, the light regime in which the algae rapidly alternate between 10 and $1500 \mu \mathrm{mol}$ photons $\mathrm{m}^{-2} \mathrm{~s}^{-1}$ leads to the same level of pigmentation. The microalgal pigment content is highly dependent on the perceived light intensity. In the process of photoacclimation, the pigment content deceases with increasing light intensity, which reaches a plateau at high light intensities (Takache et al. 2012). It could have been expected that pigment content correlates to the biomass specific light absorption rate (Sforza et al. 2015). If this was the case in our experiments, blue light should have resulted in a lower pigment content to compensate for the higher absorption capacity for blue light. Likewise, yellow light should have resulted in an increased pigment content to harvest more of the weakly absorbed yellow light. As the mechanism behind pigment acclimation in response to light quality has not yet been unravelled (Falkowski et al. 1991) and the importance of other (wavelength specific) light acclimation responses has not yet been studied in detail, our observation is difficult to explain. In literature, statements regarding pigment accumulation under different colors of light are contradictory. This is most likely due to the fact that it is difficult to distinguish between the effect of light intensity and light quality, as the color of light determines the ease of absorption and therefore the biomass specific light absorption 
rate $\left(q_{p h}\right)$. For a fair comparison, the pigmentation should be compared for cultures exposed to different colors of light, but with the same biomass specific light absorption rate, which can be challenging to achieve in photobioreactors with steep light gradients.

Remarkable is the fact that the lowest $F_{v} / F_{m}$ value was obtained for cultures exposed to yellow light $(0.49 \pm 0.04)$ while this culture yielded the highest volumetric productivity. The areal productivity for yellow light was almost double compared to blue light, where a $F_{v} / F_{m}$ value of 0.63 was measured. This suggests, therefore, that part of the photosystems (PSII) became inactive which reduced the biochemical conversion capacity, however, yellow light could still be used at a higher efficiency than, for example, blue light. However, $F_{v} / F_{m}$ values should preferably be measured with the same color of light as the color of the cultivation light as, for higher plants, it was observed that this is required to measure maximum quantum yield values (Chow et al. 1990). The rationale behind this statement is that the PSI/PSII stoichiometry is optimized for the light the plant is exposed to and when there is a sudden change in light spectrum, there might be an imbalanced excitation of the two photosystems (Terashima et al. 2009). This could have affected our results as we applied red light $(620 \mathrm{~nm})$ for our measurements.

The hypothesis of this study is that the degree of photosystem saturation dictates the photosynthetic efficiency of the microalgae culture and that photosystem saturation can be controlled by applying different colors of light. The rationale has been previously discussed in literature (Kubín et al. 1983, Terashima et al. 2009, Paradiso et al. 2011, Mattos et al. 2015) and applies to both microalgae mass cultures and to the canopy of horticulture crops. In both situations, weakly absorbed light (green or yellow) is expected to increase the photosynthetic efficiency as less energy is dissipated in the surface layer of the photobioreactor or the outer zone of the canopy. Indeed, several experimental studies demonstrated that green light supplementation led to increased productivity of crops (Kim et al. 2004, Johkan et al. 2012, Olle et al. 2013). Sforza et al. used a spectral converter filter to convert the green and yellow light to red light with the intention to maximize the portion of useful light for photosynthesis (Sforza et al. 2015). No significant improvement was found. According to our hypothesis this approach would actually decrease the productivity under high light conditions as the culture will 
become even more oversaturated. To maximize productivity in such a setup, the red and blue light should be shifted to the green range.

It remains ambiguous whether yellow light suffices for optimal growth. To our knowledge, blue light supplementation to yellow or green light has not been studied previously. Yellow light could possibly be more difficult for cultivation than green light as the green emission spectrum of some light sources partly overlaps with the blue region. In our experiments, cultures that were supplemented with a moderate amount of blue light gave a higher productivity, had more stable cultivation (i.e. less day-to-day variation in productivity), and had enhanced cell fitness as indicated by a higher $F_{v} / F_{m}$ value. The improvement in performance cannot be attributed to the energy content of the additional $50 \mu \mathrm{mol}$ photons $\mathrm{m}^{-2} \mathrm{~s}^{-1}$ of blue light, as this is only a $3.5 \%$ increase in total light intensity. This finding posits the following tentative hypothesis: blue light acts as a trigger for metabolic regulatory mechanisms that are essential for stable cultivation under the described mass culture conditions.

Higher plants were ascertained to exhibit photoprotection mechanisms that are solely activated by blue light (Gruszecki et al. 2010). Authors of the same paper also observed that blue light is exploited by plants as an indicator of over-excitation and the need to switch to a state enhancing thermal energy dissipation. In addition, for the diatom Phaeodactylum tricornutum, blue light was determined to be essential for the activation of photoprotection under high light as an increased NPQ capacity and a larger pool of xanthophyll cycle pigments could only be observed in cultures grown under blue light (Costa et al. 2013). In another study, it was hypothesized that, in Chlorella, blue light produces the same effects that are normally observed for strong white light (Wilhelm et al. 1985). Blue light is also known to affect several metabolic pathways and induce gene expression in both algae and plants via blue light receptors (Schulze et al. 2014). In horticulture, the beneficial effects of blue light supplementation have been demonstrated in several studies. Blue light supplementation was found to double the photosynthetic capacity and prevent abnormal growth in cucumber plants (Hogewoning et al. 2010). In spinach, blue light was discovered to enhance the acclimation responses to high light conditions and to increase the chlorophyll content (Matsuda et al. 2008). Other greenhouse plants were found to have increased biomass accumulation, increased vegetative growth, and expanded leafs under blue light supplementation (Olle et al. 2013). To conclude, blue light seems to play a key role in the survival and development 
of photosynthetic organisms. Also our experiments with Chlamydomonas indicate that exposure to blue light is essential for optimal growth under high light conditions, probably caused by wavelength-dependent activation of photoprotection and dissipation mechanisms.

Maintaining a stable culture under red light was difficult. Under deep red light at 1500 $\mu$ mol photons $\mathrm{m}^{-2} \mathrm{~s}^{-1}$, only one experiment out of eight was successful. Productivity was slightly lower than was estimated by our model based on the light emission spectrum of the deep red light source. Possibly, $1500 \mu \mathrm{mol}$ photons $\mathrm{m}^{-2} \mathrm{~s}^{-1}$ of deep red light was too intense for the photosystems. On the one hand, this is striking since the biomass specific light absorption rate is lower than that of blue light. On the other hand, the regulatory mechanisms triggered by the color of light seems to be more complex than initially expected. Therefore, it cannot be excluded that, under high light conditions, a balanced mix of wavelengths is required for optimal growth. At $850 \mu \mathrm{mol}$ photons $\mathrm{m}^{-2} \mathrm{~s}^{-1}$ of deep red light two experiments were successful and reproducible. At this light intensity, severe damage to the photosystems is less probable. As expected, based on our theory that strongly absorbed light decreases light use efficiency, the biomass productivity was lower than for the culture in warm white light. Also, under orange-red light at 1500 $\mu$ mol photons $\mathrm{m}^{-2} \mathrm{~s}^{-1}$, productivity was lower than our model predicted. The use of orange-red light for microalgae cultivation is common and generally without complications. Kliphuis et al. (Kliphuis et al. 2012), for example, used the same light source as we did, but worked with light intensities below $100 \mu \mathrm{mol}$ photons $\mathrm{m}^{-2} \mathrm{~s}^{-1}$. The high intensities of red and yellow light in this study have not been previously reported for Chlamydomonas. Therefore, we suggest that the high light intensity must have been the explanation for the poor performance. For cultures exposed to yellow light, blue light supplementation was found to improve reactor performance and productivity. A similar approach could possibly work for red light as well.

The cell size of Chlamydomonas is influenced by light color. Continuous blue light is known to delay cell division which signifies that cells continue to grow in size as biomass is accumulating (Münzner et al. 1992, Oldenhof et al. 2006). Otherwise stated, a larger cell size is required for cell division to occur. A blue light receptor is likely to be involved (Münzner et al. 1992). The opposite was determined for red light. Under red light, cells undergo a division cycle when they have achieved the minimal cell size 
required for division. In practice, the consequence is that, compared to white light, the average cell size is larger under blue light and smaller under red light (Kubín et al. 1983, Wilhelm et al. 1985, Kim et al. 2014). Cell size and the accompanied geometrical arrangement of the chloroplast, as well as the cellular chlorophyll content, are all factors that may influence light penetration and light scattering. This phenomenon, therefore, complicates modelling reactor productivity. Also our productivity measurements may have been influenced by this unintended effect of blue and red light.

\subsection{Conclusions}

In this study, we presented areal biomass productivities of high density microalgae cultures exposed to high light intensities of different colors. Tubidostat control ensured that the total amount of absorbed light was equal for each color. Our results demonstrate that, under mass culture conditions, biomass productivity and the biomass specific light absorption rate are inversely correlated as oversaturation of the photosystems leads to a waste of light energy and, therefore, a lower biomass yield on light. Highest biomass productivity, measured under continuous illumination, was obtained employing yellow light $\left(54 \mathrm{~g} \mathrm{~m}^{-2} \mathrm{~d}^{-1}\right)$, closely followed by cultures grown under warm white light $\left(50 \mathrm{~g} \mathrm{~m}^{-2} \mathrm{~d}^{-1}\right)$. Cultivation under blue, orange-red, and deep red light resulted in biomass productivities of approximately $29 \mathrm{~g} \mathrm{~m}^{-2} \mathrm{~d}^{-1}$ which is nearly half of the productivity measured for yellow light. The microalgae absorption cross section remained the same under all tested conditions. Our approach with different colors of light to investigate photosystem saturation was interfered by intrinsic biological effects. Cultivation under pure yellow light was impeded. Minimal supplementation of blue light to the cultures in yellow light was determined to stimulate normal growth and increase productivity. Additional research is required to reveal the underlying mechanism that is responsible for the beneficial effects of blue light supplementation. Taking into account possible wavelength deficiencies, white light with a high green or yellow content in addition to a small blue fraction would result in the highest productivity of microalgae mass cultures. This study provides a solid base for further research on decreasing the biomass specific light absorption in order to maximize productivity. Presently, the creation of antenna size mutants that permanently absorb less light per cell is the most promising solution. 


\section{Appendix A: Microalgae growth model for reactor productivity estimation}

A kinetic model has been adopted to be able to predict the biomass productivity in a photobioreactor as a function of the local light intensity and light spectrum. The model comprises two compartments: the chloroplast and the cell content outside the chloroplast. In the chloroplast, 3-carbon sugars (triose) are produced. Here, we express photosynthetic sugar production in units of the 1-carbon sugar equivalent $\mathrm{CH}_{2} \mathrm{O}$ that was formed. Sugar production in the chloroplast $\left(q \mathcal{C}_{\mathrm{H} 2 \mathrm{O}}, \operatorname{mol}_{\mathrm{CH} 2 \mathrm{O}} \mathrm{mol}_{\mathrm{x}}{ }^{-1} \mathrm{~s}^{-1}\right)$ is driven by light energy and can be described by the hyperbolic tangent model of Jassby and Platt (Jassby et al. 1976):

$q_{C H 2 O}^{c}=q_{C H 2 O, m}^{c} \cdot \int_{0}^{d} \tanh \left(\frac{\sum_{\lambda=400}^{\lambda=700} Y_{C H 2 O} / p h, m, \lambda \cdot q_{p h, \lambda}(z) \cdot \Delta \lambda}{q_{C H 2 O, m}^{c}}\right) \cdot d z$

In this equation, $q_{\text {Hнг, }}$ is the maximal sugar production rate $\left(\mathrm{mol}_{\mathrm{CH} 2 \mathrm{O}} \mathrm{mol}_{\mathrm{x}}{ }^{-1} \mathrm{~S}^{-1}\right)$ in the chloroplast; $Y_{C H 2 O / p h, m, \lambda}$ is the maximum yield of sugar on light energy $\left(\operatorname{mol}_{\mathrm{CH} 2 \mathrm{O}} \mathrm{mol}_{\mathrm{ph}}{ }^{-1}\right)$ in the event that there would be no oversaturation and $q_{p h, \lambda}(\mathrm{z})$ is the biomass specific light absorption rate $\left(\mathrm{mol}_{\mathrm{ph}} \mathrm{mol}_{\mathrm{x}}^{-1} \mathrm{~S}^{-1}\right)$ at reactor depth $z$, which is defined as follows in equation A2:

$q_{p h, \lambda}(z)=a_{x, \lambda} \cdot I_{p h, \lambda}(z)=\frac{I_{p h, \lambda}(z-d z)-I_{p h, \lambda}(z)}{C_{x} \cdot d z}$

Where $I_{p h, \lambda}(\mathrm{z})$ is local light intensity at reactor depth $z ; C_{X}$ is the biomass concentration in the reactor. $I_{p h, \lambda}(\mathrm{z})$ is calculated by the following equation according to the Lambert-Beer law:

$I_{p h, \lambda}(z)=I_{p h, \lambda}(0) \cdot e^{-a_{x, \lambda} \cdot C_{x} \cdot z \cdot f_{l}}$

In this equation, $I_{p h, \lambda}(0)$ is the incident light intensity at the illuminated surface of the reactor, $a_{x, \lambda}\left(\mathrm{m}^{2} \mathrm{~mol}_{\mathrm{x}}{ }^{-1}\right)$ is the biomass absorption cross section and $f_{l}$ the light path correction factor. This factor accounts for the influence of light scattering within the microalgae suspension and the fact that the incident light is not perfectly collimated. The effect is that the path travelled by the light rays within the algae suspension is longer than would have been the case for light entering perpendicular to the reactor surface and with the absence of light scattering within the microalgae suspension. A longer light path increases the probability of light absorption and therefore the $f_{l}$ factor increases the 
accuracy of the Lambert-Beer equation shown above. The $f_{l}$ parameter (Table A1) was fitted to the actual light intensity measurements for each light color separately using the data shown in Appendix C. The $f_{l}$ value of sunlight was assumed to be equal to that of warm white light.

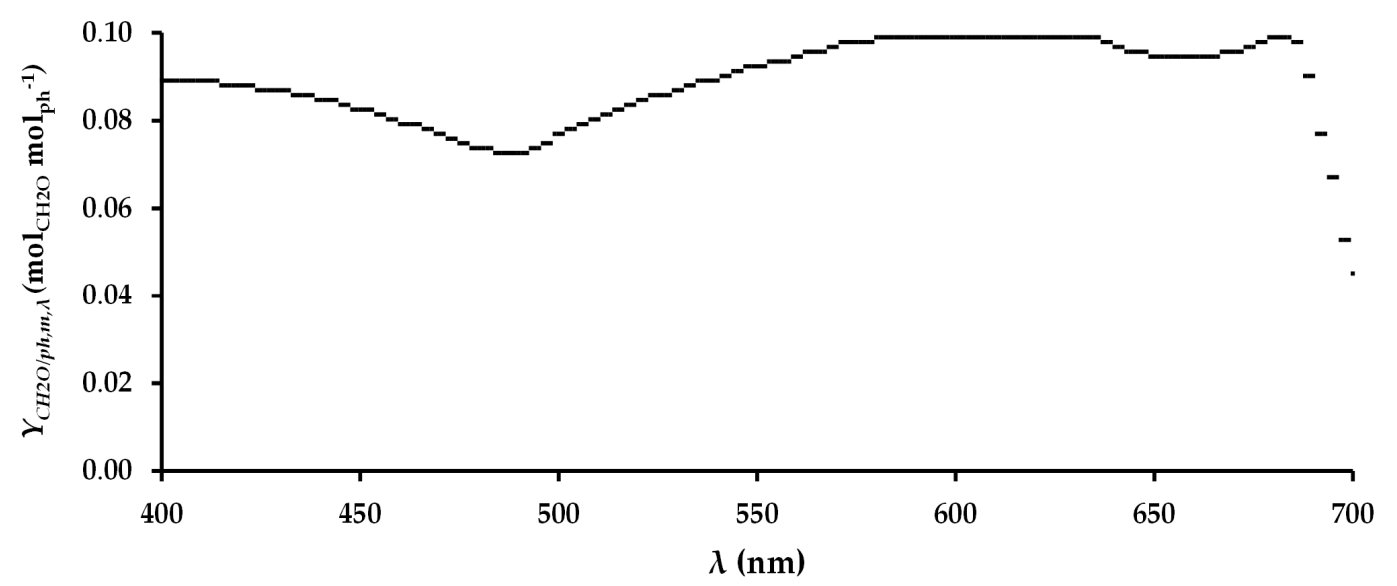

Fig. A.1 Maximum yield of sugar on light energy ( mol $_{\mathrm{CH} 2 \mathrm{O}} \mathrm{mol}_{\mathrm{ph}}{ }^{-1}$ ) as measured for every wavelength by Emerson and Lewis (Emerson et al. 1943). The original values have been multiplied by a factor of 1.1 to bring them in line with results obtained by other researchers, as discussed in the text. Numerical values for $Y_{C H 2 O / p h, m, \lambda}$ can be found online in the supplementary material (de Mooij et al. 2016).

The cell content minus the chloroplast was defined as the second compartment of this model. Here, part of the 3-carbon sugar is used for the production of new biomass at specific growth rate $\mu$. Another part of the sugar is respired in the mitochondria to provide energy in the form of ATP to support the growth reactions and to fulfil the maintenance requirements. The following equation was derived from a substrate balance over the cell minus chloroplast compartment, where the maintenance requirement is fulfilled by substrate consumption according to Pirt (1965).

$\mu=\left(q_{C H 2 O}^{c}-m_{C H 2 O}\right) \cdot Y_{x / C H 2 O}$

and:

$\mu_{m}=\left(q_{C H 2 O, m}^{c}-m_{C H 2 O}\right) \cdot Y_{x / C H 2 O}$

Here, $m_{C H 2 O}$ is the biomass specific maintenance rate $\left(\operatorname{mol}_{\mathrm{CH} 2 \mathrm{O}} \operatorname{mol}_{\mathrm{x}}{ }^{-1} \mathrm{~s}^{-1}\right) ; Y_{X} / \mathrm{CH} 2 \mathrm{O}$ is the biomass yield on sugar $\left(\mathrm{mol}_{\mathrm{x}} \mathrm{mol}_{\mathrm{CH} 2 \mathrm{O}^{-1}}\right)$; and $\mu_{m}$ is the maximum biomass specific growth rate $\left(\mathrm{s}^{-1}\right)$. 
Using equation A.1 through A4, $\mu$ can be computed for every light intensity $I_{p h}$ at every location inside a flat panel photobioreactor culture illuminated from one side. Values for $\mathrm{a}_{\mathrm{x}, \lambda}, I_{p h, i n}, Y_{C H 2 O / p h, m, \lambda} q_{C H 2 O, m}^{C}$ and $m_{C H 2 O}$ should be provided (see Table A1). The integral in eq. A1 is easily solved by numerical integration over a large number of sublayers $(N)$ with depth $\Delta z$. This procedure was implemented in a MS Excel based calculation routine and the use of 100 sublayers was demonstrated to result in sufficient accuracy of the model prediction.

The biomass yield on light energy at each point in the reactor $\left(Y_{x / p h}(\mathrm{z}), \mathrm{mol}_{\mathrm{x}} \mathrm{mol}_{\mathrm{ph}}{ }^{-1}\right) \mathrm{can}$ now also be calculated in the same manner by using the following equation:

$$
Y_{x / p h}(z)=\frac{\mu(z)}{q_{p h}(z)}
$$

The areal biomass productivity $\left(r_{x}, \mathrm{~g} \mathrm{~m}^{-2} \mathrm{~d}^{-1}\right)$ in a photobioreactor is the product of the biomass concentration $\left(C_{x}, \operatorname{mol}_{x} \mathrm{~L}^{-1}\right)$, reactor depth $d$, and the average biomass specific growth rate $\bar{\mu}$ :

$r_{x}=C_{x} \cdot d \cdot \bar{\mu}$

Using numerical integration, for a selected value of $C_{x}$, the corresponding $r_{x}$ can be obtained. To maximize productivity, a biomass concentration has to be found such, that at the back of the reactor the maintenance rate $\left(m_{\mathrm{CH} 2 \mathrm{O}}\right)$ equals the local sugar production rate $\left(q_{\text {Сноо }}(d)\right)$. In this situation, the light intensity at the backside is equal to the compensation point and the net photosynthesis rate is equal to zero. This means that at all locations in the photobioreactor, the biomass is positively contributing to biomass productivity and there is no dark zone. Using an iteration method, $r_{X}$ can be maximized by incrementally changing the $C_{x}$ value for every repetition until it has converged to the optimal value. In this study the MS Excel 'Solver' (GRG nonlinear algorithm) was employed.

In the model it is assumed that under low light conditions, light is used at maximum efficiency. This is expressed using the parameter $\left(Y_{C H 2 O / p h, m, \lambda}\right.$, which is the maximum yield of sugar on light energy $\left(\mathrm{mol}_{\mathrm{CH} 2 \mathrm{O}} \mathrm{mol}_{\mathrm{ph}^{-1}}\right)^{-1}$. As the maximum yield on light is wavelength dependent, we incorporated the $Y_{C H 2 O / p h, m, \lambda}$ values as measured by Emerson and Lewis (1943) into the model. All of their $Y_{C H 2 O / p h, m, \lambda}$ values have been multiplied with 
a factor 1.1 to bring them more in line with the results obtained by a number of other researchers (Tanada 1951, Björkman et al. 1987, Evans 1987, Malkin et al. 1996, Sforza et al. 2015) who also investigated the maximum quantum yield and, overall, ascertained higher values compared to Emerson and Lewis. See Fig. A1 for the value of $Y_{C H 2 O / p h, m, \lambda}$ as a function of wavelength. The fact that the small differences in $Y_{C H 2 O / p h, m, \lambda}$ had a minimal effect on the estimated productivity signifies that the effect of $Y_{C H 2 O / p h, m, \lambda}$ is of minor importance for the productivity of a reactor exposed to high light conditions. Instead, oversaturation of photosynthesis is believed to be the dominant phenomena.

Table A1 Overview of the model parameters.

\begin{tabular}{|c|c|c|c|c|}
\hline Parameter & Value & Unit & Description & References \\
\hline$\mu_{m}$ & 0.132 & $h^{-1}$ & maximal biomass specific growth rate & (Janssen et al. 2000) \\
\hline$q \varepsilon_{H 20, m}$ & $6.17 \cdot 10^{-5}$ & $\operatorname{mol}_{\mathrm{CH} 2 \mathrm{O}} \operatorname{mol}_{\mathrm{x}}^{-1} \mathrm{~S}^{-1}$ & $\begin{array}{l}\text { maximal 3-carbon sugar production } \\
\text { rate; calculated from } \mu_{m} \text { using eq. A5. }\end{array}$ & \\
\hline$Y_{C H 2 O / p h, m u \lambda}$ & See Fig. A1 & $\operatorname{mol}_{\mathrm{CH} 2 \mathrm{O}} \mathrm{mol}_{\mathrm{ph}^{-1}}$ & $\begin{array}{c}\text { Maximum yield of sugar on light } \\
\text { energy }\end{array}$ & $\begin{array}{l}\text { (Emerson and } \\
\text { Lewis, 1943) }\end{array}$ \\
\hline$m_{\mathrm{CH} 2 \mathrm{O}}$ & $3.5 \cdot 10^{-6}$ & $\operatorname{mol}_{\mathrm{CH} 2 \mathrm{O}} \operatorname{mol}_{\mathrm{x}}{ }^{-1} \mathrm{~S}^{-1}$ & biomass specific maintenance rate & $\begin{array}{l}\text { (Kliphuis et al. } \\
\text { 2012) }\end{array}$ \\
\hline$Y_{X / \mathrm{CH} 2 \mathrm{O}}$ & 0.63 & $\operatorname{mol}_{\mathrm{X}} \operatorname{mol}_{\mathrm{CH} 2 \mathrm{O}^{-1}}$ & $\begin{array}{l}\text { biomass yield on 3-carbon sugar using } \\
\text { ammonia as N-source }\end{array}$ & $\begin{array}{l}\text { (Kliphuis et al. } \\
\text { 2012) }\end{array}$ \\
\hline$a_{x, \lambda}$ & - & $m^{2} g^{-1}$ & absorption cross section & $\begin{array}{l}\text { (de Mooij et al. } \\
\text { 2016) }\end{array}$ \\
\hline$M_{x}$ & 24 & $\mathrm{~g} \mathrm{~mol}_{\mathrm{x}}^{-1}$ & $\begin{array}{l}\text { Biomass dry weight to } \\
\text { C-mol conversion factor }\end{array}$ & (Duboc et al. 1999) \\
\hline$f_{l}$ & $\begin{array}{r}\text { B: } 1.17 \\
\text { OR: } 1.45 \\
\text { DR: } 1.60 \\
\text { Y: } 1.57 \\
\text { W: } 1.77\end{array}$ & - & Light path correction factor & This paper \\
\hline
\end{tabular}




\section{Appendix B: Model estimation values}

Table B1 Overview of all model estimation values of the areal biomass productivity $\left(r_{x}\right)$, biomass concentration $\left(C_{x}\right)$, and biomass specific growth rate $(\mu)$ at $I_{p h, i n}=1500 \mu \mathrm{mol}$ photons $\mathrm{m}^{-2} \mathrm{~s}^{-1}$ for different light colors.

\begin{tabular}{lccc}
\hline Light color & $\boldsymbol{r}_{\boldsymbol{x}}\left(\mathbf{g ~ m}^{-2} \mathbf{d}^{-1}\right)$ & $\boldsymbol{C}_{\boldsymbol{x}}(\mathbf{g ~ \mathbf { L }}-\mathbf{1})$ & $\boldsymbol{D}(\mathbf{d}-\mathbf{1})$ \\
\hline Warm white & 50.7 & 2.3 & 1.56 \\
Sunlight & 44.2 & 2.1 & 1.48 \\
Yellow & 63.2 & 2.8 & 1.64 \\
Deep red & 33.5 & 1.3 & 1.83 \\
Orange-red & 47.5 & 1.9 & 1.78 \\
Blue & 27.4 & 1.0 & 1.89 \\
\hline
\end{tabular}




\section{Appendix C: light measurements and experimental results}

Table C1 Overview of light measurements and experimental results for all colors of light. Colors are abbreviated as follows: $B=$ Blue, $O R=$ Orange-red, $Y B=$ Yellow $+B l u e, D R=$ Deep red, $W=$ Warm white, $Y$ = Yellow. $N$ represents the number of days of measurement.

\begin{tabular}{lcccccccccc}
\hline Experiment & $\mathbf{1}$ & $\mathbf{2}$ & $\mathbf{3}$ & $\mathbf{4}$ & $\mathbf{5}$ & $\mathbf{6}$ & $\mathbf{7}$ & $\mathbf{8}$ & $\mathbf{9}$ & $\mathbf{1 0}$ \\
\hline \hline Light color & $\mathrm{B}$ & $\mathrm{B}$ & $\mathrm{B}$ & OR & OR & OR & DR & YB & YB & YB \\
Iph,in $\left(\mu \mathrm{mol} \mathrm{m}^{-2} \mathrm{~s}^{-1}\right)$ & 1509 & 1490 & 1501 & 1509 & 1499 & 1501 & 1493 & $1436+51$ & $1436+51$ & $1447+46$ \\
Iph,out $\left(\mu \mathrm{mol} \mathrm{m}^{-2} \mathrm{~s}^{-1}\right)$ & 10 & 10 & 11 & 13 & 13 & 10 & 12 & 11 & 11 & 10 \\
$\mathrm{~N}$ & 8 & 4 & 6 & 6 & 6 & 7 & 6 & 8 & 4 & 5 \\
$C_{x}\left(\mathrm{~g} \mathrm{~L}^{-1}\right)$ & 0.96 & 0.90 & 0.94 & 1.80 & 1.66 & 1.86 & 1.14 & 2.72 & 2.93 & 3.21 \\
$\mathrm{SD}$ & 0.06 & 0.04 & 0.036 & 0.10 & 0.09 & 0.03 & 0.06 & 0.16 & 0.05 & 0.09 \\
$D\left(\mathrm{~d}^{-1}\right)$ & 2.09 & 2.23 & 2.10 & 1.03 & 1.20 & 1.09 & 1.93 & 1.34 & 1.31 & 1.29 \\
$\mathrm{SD}$ & 0.05 & 0.03 & 0.12 & 0.04 & 0.19 & 0.02 & 0.17 & 0.19 & 0.02 & 0.05 \\
$r_{x}\left(\mathrm{~g} \mathrm{~m}^{-2} \mathrm{~d}^{-1}\right)$ & 28.5 & 29.6 & 28.3 & 26.4 & 28.4 & 28.9 & 30.9 & 51.7 & 54.8 & 56.6 \\
$\mathrm{SD}$ & 2.1 & 1.1 & 1.5 & 1.5 & 3.6 & 0.6 & 1.9 & 6.2 & 1.2 & 2.5 \\
$Y_{x / p h}\left(\mathrm{~g} \mathrm{~mol}_{\mathrm{ph}, \mathrm{in}^{-1}}\right)$ & 0.22 & 0.23 & 0.22 & 0.20 & 0.22 & 0.22 & 0.24 & 0.42 & 0.44 & 0.45 \\
$\mathrm{SD}$ & 0.02 & 0.01 & 0.01 & 0.01 & 0.03 & 0.00 & 0.02 & 0.05 & 0.01 & 0.02 \\
\hline
\end{tabular}

\begin{tabular}{lccccccccc}
\hline Experiment & $\mathbf{1 1}$ & $\mathbf{1 2}$ & $\mathbf{1 3}$ & $\mathbf{1 4}$ & $\mathbf{1 5}$ & $\mathbf{1 6}$ & $\mathbf{1 7}$ & $\mathbf{1 8}$ & $\mathbf{1 9}$ \\
\hline \hline Light color & $\mathrm{W}$ & $\mathrm{W}$ & $\mathrm{W}$ & $\mathrm{DR}$ & $\mathrm{DR}$ & $\mathrm{Y}$ & $\mathrm{Y}$ & $\mathrm{Y}$ & $\mathrm{Y}$ \\
Iph,in $\left(\mu \mathrm{mol} \mathrm{m}^{-2} \mathrm{~s}^{-1}\right)$ & 1485 & 1488 & 1486 & 836 & 838 & 1495 & 1476 & 1510 & 1514 \\
Iph,out $\left(\mu \mathrm{mol} \mathrm{m}^{-2} \mathrm{~S}^{-1}\right.$ & 9 & 9 & 9 & 11 & 11 & 10 & 10 & 9 & 11 \\
$\mathrm{~N}$ & 12 & 12 & 6 & 5 & 9 & 13 & 9 & 13 & 12 \\
$C_{x}\left(\mathrm{~g} \mathrm{~L}^{-1}\right)$ & 2.57 & 2.45 & 2.67 & 1.05 & 1.11 & 3.61 & 3.64 & 3.83 & 3.21 \\
$\mathrm{SD}$ & 0.06 & 0.06 & 0.05 & 0.07 & 0.11 & 0.32 & 0.18 & 0.37 & 0.35 \\
$D\left(\mathrm{~d}^{-1}\right)$ & 1.36 & 1.45 & 1.26 & 1.16 & 1.16 & 0.78 & 0.71 & 0.75 & 0.68 \\
$\mathrm{SD}$ & 0.03 & 0.06 & 0.03 & 0.06 & 0.09 & 0.27 & 0.12 & 0.10 & 0.26 \\
$r_{x}\left(\mathrm{~g} \mathrm{~m}^{-2} \mathrm{~d}^{-1}\right)$ & 49.5 & 50.6 & 47.7 & 17.6 & 18.6 & 39.9 & 37.1 & 41.4 & 30.8 \\
$\mathrm{SD}$ & 1.5 & 2.0 & 1.8 & 0.8 & 2.9 & 13.3 & 5.5 & 7.6 & 12.5 \\
$Y_{x / p h}\left(\mathrm{~g} \mathrm{~mol}_{\left.\mathrm{ph}, \mathrm{in}^{-1}\right)}\right.$ & 0.39 & 0.39 & 0.37 & 0.24 & 0.26 & 0.31 & 0.29 & 0.32 & 0.24 \\
$\mathrm{SD}$ & 0.01 & 0.02 & 0.01 & 0.01 & 0.04 & 0.10 & 0.04 & 0.06 & 0.10 \\
\hline
\end{tabular}




\section{Appendix D: Light intensity distribution of light sources}

Table D1 Warm white incident light intensity ( $\mu$ mol photons $\left.\mathrm{m}^{-2} \mathrm{~s}^{-1}\right)$ distribution over the reactor surface. The light was measured at 28 fixed points evenly distributed over the light exposed surface of the front glass panel of the culture chamber. The light was measured behind the front glass panel of a dummy reactor that lacks the other glass panels and reactor components, to make light measurements convenient. For this measurement, the average light intensity was 1501 umol photons $\mathrm{m}^{-2} \mathrm{~s}^{-1}$. Using colors the intensity distribution is visualized (darker = higher intensity).

\begin{tabular}{llll}
\hline 1235 & 1429 & 1352 & 1286 \\
\hline 1464 & 1665 & 1656 & 1409 \\
\hline 1683 & 1837 & 1737 & 1739 \\
1586 & 1882 & 1953 & 1571 \\
1601 & 1738 & 1670 & 1438 \\
1383 & 1568 & 1591 & 1177 \\
1001 & 1135 & 1144 & 1099 \\
\hline
\end{tabular}

Table D2 Blue incident light intensity ( $\mu \mathrm{mol}$ photons $\mathrm{m}^{-2} \mathrm{~s}^{-1}$ ) distribution over the reactor surface. The light was measured at 28 fixed points evenly distributed over the light exposed surface of the front glass panel of the culture chamber. The light was measured behind the front glass panel of a dummy reactor that lacks the other glass panels and reactor components, to make light measurements convenient. For this measurement, the average light intensity was $1489 \mu \mathrm{mol}$ photons $\mathrm{m}^{-2} \mathrm{~s}^{-1}$. Using colors the intensity distribution is visualized (darker = higher intensity).

\begin{tabular}{|c|c|c|c|}
\hline 1442 & 1493 & 1372 & 1386 \\
\hline 1488 & 1361 & 1492 & 1685 \\
\hline 1656 & 1618 & 1600 & 1592 \\
\hline 1459 & 1579 & 1573 & 1656 \\
\hline 1474 & 1488 & 1548 & 1270 \\
\hline 1397 & 1547 & 1413 & 1435 \\
\hline 1488 & 1317 & 1488 & 1379 \\
\hline
\end{tabular}


Table D3 Deep red incident light intensity ( $\mu \mathrm{mol}$ photons $\mathrm{m}^{-2} \mathrm{~s}^{-1}$ ) distribution over the reactor surface. The light was measured at 28 fixed points evenly distributed over the light exposed surface of the front glass panel of the culture chamber. The light was measured behind the front glass panel of a dummy reactor that lacks the other glass panels and reactor components, to make light measurements convenient. For this measurement, the average light intensity was $1503 \mu \mathrm{mol}$ photons $\mathrm{m}^{-2} \mathrm{~s}^{-1}$. Using colors the intensity distribution is visualized (darker = higher intensity).

\begin{tabular}{lccc}
\hline 1303 & 1310 & 1251 & 1230 \\
1604 & 1590 & 1619 & 1599 \\
1620 & 1602 & 1686 & 1573 \\
1614 & 1700 & 1681 & 1674 \\
1631 & 1648 & 1628 & 1203 \\
1542 & 1592 & 1608 & 1499 \\
\hline 1232 & 1289 & 1302 & 1269 \\
\hline
\end{tabular}

Table D4 Yellow incident light intensity ( $\mu$ mol photons $\mathrm{m}^{-2} \mathrm{~s}^{-1}$ ) distribution over the reactor surface. The light was measured at 28 fixed points evenly distributed over the light exposed surface of the front glass panel of the culture chamber. The light was measured behind the front glass panel of a dummy reactor that lacks the other glass panels and reactor components, to make light measurements convenient. For this measurement, the average light intensity was $1504 \mu \mathrm{mol}$ photons $\mathrm{m}^{-2} \mathrm{~s}^{-1}$. Using colors the intensity distribution is visualized (darker = higher intensity).

\begin{tabular}{llll}
\hline 1389 & 1469 & 1358 & 1380 \\
1521 & 1614 & 1521 & 1524 \\
1593 & 1604 & 1532 & 1493 \\
1650 & 1613 & 1606 & 1487 \\
1628 & 1565 & 1498 & 1320 \\
1582 & 1533 & 1486 & 1494 \\
1367 & 1409 & 1357 & 1357 \\
\hline
\end{tabular}


Table D5 Orange-red incident light intensity ( $\mu$ mol photons $\mathrm{m}^{-2} \mathrm{~s}^{-1}$ ) distribution over the reactor surface. The light was measured at 28 fixed points evenly distributed over the light exposed surface of the front glass panel of the culture chamber. The light was measured behind the front glass panel of a dummy reactor that lacks the other glass panels and reactor components, to make light measurements convenient. For this measurement, the average light intensity was 1512 umol photons $\mathrm{m}^{-2} \mathrm{~s}^{-1}$. Using colors the intensity distribution is visualized (darker = higher intensity).

\begin{tabular}{lccc}
\hline 1231 & 1201 & 1120 & 1109 \\
1568 & 1647 & 1602 & 1582 \\
1781 & 1742 & 1672 & 1665 \\
1532 & 1618 & 1611 & 1483 \\
1514 & 1524 & 1650 & 1642 \\
1490 & 1413 & 1507 & 1468 \\
1530 & 1540 & 1540 & 1483 \\
\hline
\end{tabular}


Chapter 3 



\section{Chapter 4:}

\section{Effect of photoacclimation on microalgae mass culture productivity}

Tim de Mooij, Zeynab Rajabali Nejad, Lennard van Buren, René H. Wijffels, Marcel Janssen 


\begin{abstract}
Microalgae are capable of adapting their pigmentation to the light regime to which they are exposed. In high density microalgae cultures exposed to sunlight, the high pigment content leads to oversaturation of the photosystems resulting in increased light energy dissipation at the reactor surface, reducing the light use efficiency. In theory, therefore, pigment reduction would maximize biomass productivity. In this study, we have measured the long-term biomass productivity and short-term oxygen production rate of low-pigmented cells of Chlorella sorokiniana under mass culture conditions. Reduced pigmentation was obtained through the natural process of photoacclimation under high irradiance. During the time that the pigmentation was reduced, mass culture productivity, light absorption, and light use efficiency were investigated. Photoacclimation kinetics were investigated in a light shift experiment in which the increase in absorption cross section was followed in time upon a shift from high to low light intensity. Improved productivity of low-pigmented cells under mass culture conditions was not observed in any of the experiments. There is no solid explanation based on the experimental data. The most likely explanations are that thermal dissipation mechanisms were still activated and that the photoacclimation process itself consumed a substantial amount of energy at cost of growth processes. It is suggested that photoacclimation can only be exploited in the situation that microalgal cells are grown at a fixed position (e.g. in a biofilm, or multicompartment reactor) without being exposed to rapid light fluctuations.
\end{abstract}




\subsection{Introduction}

The counterproductive photoacclimation behavior of high density microalgae cultures is an inevitable bottleneck in the optimization of the photosynthetic efficiency. Microalgae have the ability to adapt their pigmentation to the light regime to which they are exposed (Falkowski et al. 1991). Under mass culture conditions, microalgae increase their pigmentation, i.e., antenna size, in order to absorb as much light energy as possible. Higher pigmentation is beneficial in the darker zones of the culture but effectuates extensive photosystem (PS) oversaturation of the microalgal cells at the high light exposed side of the reactor. The excess amount of light energy is wastefully dissipated as heat and fluorescence (Demmig-Adams et al. 1992). In theory a decrease instead of an increase in pigmentation would maximize productivity. With a low pigment content, microalgae would be less prone to oversaturation and subsequently show an improved performance at the high light exposed side of the photobioreactor where, normally, most of the light energy is wasted. In our previous study (de Mooij et al. 2016), we demonstrated that productivity is, indeed, inversely correlated to the amount of light that is absorbed per cell and that this can be investigated by exploiting light with a spectral composition that minimizes, or maximizes, light absorption.

Photoacclimation may be exploited to improve the productivity of microalgae cultivation systems. The experiment of Grobbelaar et al. (2003) indeed demonstrated that, if microalgae are cultivated in a multicompartment reactor, and were thus allowed to acclimate to the light regime in each compartment, productivity is higher compared to a single reactor compartment with a steep light gradient going from full sunlight to complete darkness (Grobbelaar et al. 2003). This indicates that, if algae are grown in thin compartments or at a fixed location (immobilized in a biofilm), the occurrence of photoacclimation allows microalgae to make more efficient use of the light energy (Li et al. 2016).

From a theoretical point of view, the photoacclimation process could also be exploited to study the potential of hypothetical antenna size mutants under mass culture conditions. For example mutants have been created that have a locked-in high light phenotype (Bailey et al. 2013). In the past, several light shift-down experiments were performed in batch systems to study the dynamics of photoacclimation (Sukenik et al. 
1990, Melis et al. 1998, Neidhardt et al. 1998). It was extrapolated that a two to three times higher productivity could be obtained by employing low-pigmented microalgae if the negative effects that are related to high light damage could be eliminated. However, in batch experiments, biomass productivity cannot be measured in a reliable manner as there is no balanced growth when the light regime is not constant and is dictated by biomass growth itself. Therefore, we developed two continuous experimental setups in which productivity of low-pigmented microalgae was examined under well controlled mass culture conditions resulting in balanced growth.

In this work, we have measured the long-term biomass productivity and short-term oxygen production rate of low-pigmented cells of Chlorella sorokiniana under mass culture conditions. By using optimized experimental setups it was prevented that the high light acclimated algae reverted back to their high pigmented state after being exposed to the darker mass culture conditions. In the first experimental setup, high light acclimated biomass was continuously pumped into a mass culture reactor. The retention time of biomass in the reactor was sufficiently short to maintain a lower pigment content than observed in a normal cultivation with biomass that is allowed to acclimate to the mass culture conditions. In the second experimental setup, cultures with high light acclimated microalgae were analyzed for their short-term oxygen production rate in a biological oxygen monitor (BOM) in which a mass culture was simulated. In both setups, the productivity of the low-pigmented cultures was measured over several days in flat panel photobioreactors operated in turbidostat mode. In an additional experiment, pigment accumulation kinetics were investigated. The increase in absorption cross section was followed in time upon a light shift from high to low light conditions. Model-based calculations served as the theoretical foundation for our hypothesis regarding decreased oversaturation of low-pigmented cells. By comparing the experimental productivity of low-pigmented cells with that of normally pigmented cells, it was investigated whether the use of high light acclimated cells with low pigmentation could indeed result in an increased light use efficiency under mass culture conditions. 


\subsection{Material and methods}

\subsubsection{Organism and medium}

Chlorella sorokiniana CCAP 211/8k (Sorokin et al. 1953) was obtained from the American Type Culture Collection (ATCC). The algae were cultivated in filter sterilized (pore size $0.2 \mu \mathrm{m}$ ) M8-a medium with the following composition (in $\mathrm{g} \mathrm{L}^{-1}$ ): urea, 1.80; $\mathrm{KH}_{2} \mathrm{PO}_{4}, 1.48 ; \mathrm{Na}_{2} \mathrm{HPO}_{4} \cdot 2 \mathrm{H}_{2} \mathrm{O}, 0.52 ; \mathrm{MgSO}_{4} \cdot 7 \mathrm{H} 2 \mathrm{O}, 0.40 ; \mathrm{CaCl}_{2} \cdot 2 \mathrm{H}_{2} \mathrm{O}, 0.013$; EDTA ferric sodium salt, 0.116; $\mathrm{Na}_{2}$ EDTA $2 \mathrm{H}_{2} \mathrm{O}, 0.0372 ; \mathrm{H}_{3} \mathrm{BO}_{3}, 6.18 \cdot 10^{-5} ; \mathrm{MnCl}_{2} \cdot 4 \mathrm{H}_{2} \mathrm{O}, 0.013$; $\mathrm{ZnSO}_{4} \cdot 2 \mathrm{H}_{2} \mathrm{O}, 3.2 \cdot 10^{-3} ; \mathrm{CuSO}_{4}, 1.83 \cdot 10^{-3}$. The cultures were pre-cultivated in $250 \mathrm{~mL}$ shake flasks containing $100 \mathrm{~mL}$ of medium. The cultures that were employed to inoculate the short retention time reactor were maintained at $\mathrm{pH} 6.7,25{ }^{\circ} \mathrm{C}$ and were exposed to a continuous light intensity of $120-150 \mu \mathrm{mol}$ photons $\mathrm{m}^{-2} \mathrm{~s}^{-1}$. One or two days prior to inoculation, the cultures were placed in another incubator at $400-500$ $\mu \mathrm{mol}$ photons $\mathrm{m}^{-2} \mathrm{~s}^{-1}$ and at $37{ }^{\circ} \mathrm{C}$ in order to pre-acclimate the algae to the reactor conditions. The cultures used to inoculate the reactors for the biological oxygen monitor experiments were maintained at $\mathrm{pH} 7$ at $25{ }^{\circ} \mathrm{C}$ under a $16 / 8 \mathrm{~h}$ day/night cycle and a light intensity of 30-40 $\mu \mathrm{mol}$ photons $\mathrm{m}^{-2} \mathrm{~s}^{-1}$.
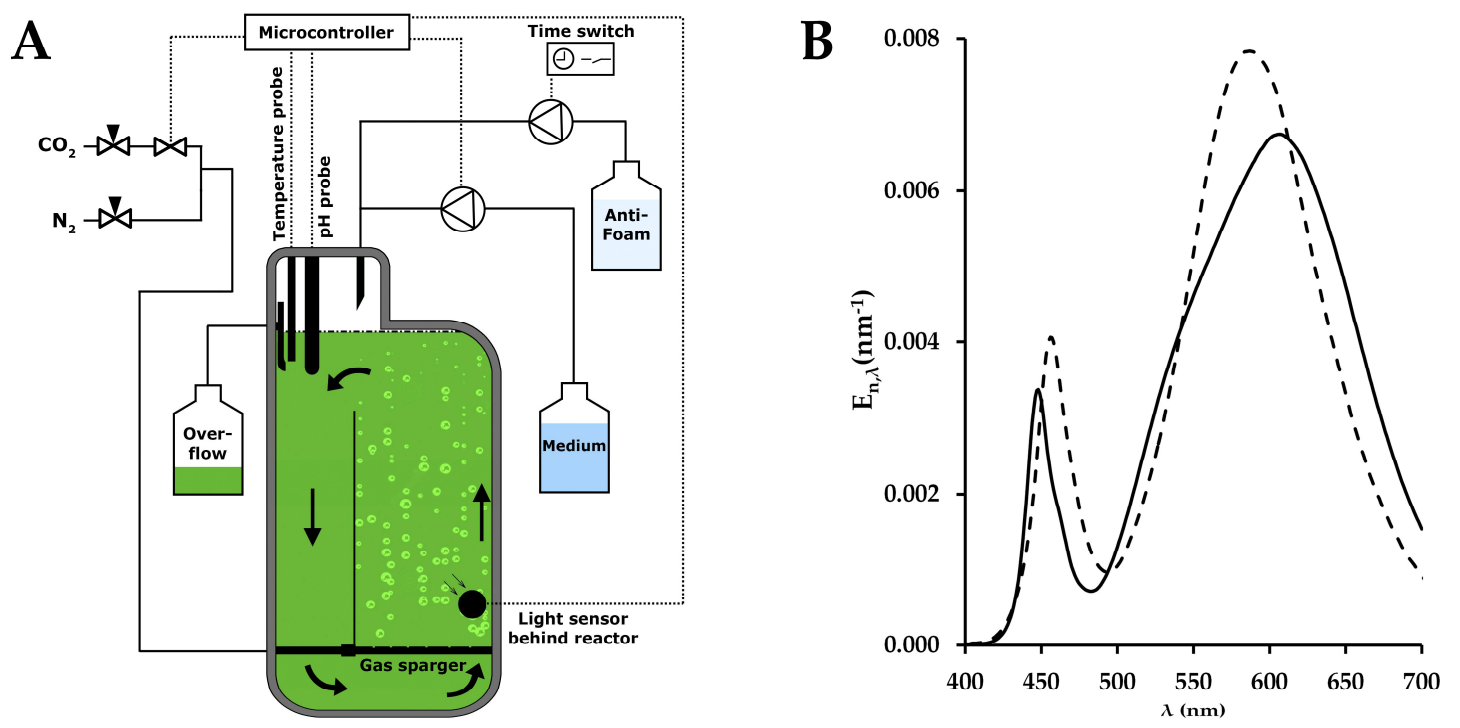

Fig. 4.1 (A) Schematic overview of the experimental setup. (B) Solid line: emission spectrum of the warm-white LEDs (BXRA W1200, Bridgelux, Livermore, USA) of the Algaemist. Dashed line: emission spectrum of the additional LED light source (warm white 45mil chip, Bridgelux, Livermore, CA). The parameter $E_{n, \lambda}$ represents the relative fraction of PAR photons present within a $1 \mathrm{~nm}$ wavelength interval. 


\subsubsection{Basic photobioreactor setup and operation}

Microalgae were continuously cultivated in flat-panel airlift photobioreactors (Algaemist, Technical Development Studio, Wageningen University, the Netherlands) with a working volume of $0.4 \mathrm{~L}$, an optical depth of $14 \mathrm{~mm}$, and an illuminated area of $0.028 \mathrm{~m}^{2}$ (See Fig. 4.1A for a schematic overview). The reactors were equipped with a black cover on the backside to prevent exposure to ambient light. Warm white light was provided by 6 Bridgelux LED lamps (BXRA W1200, Bridgelux, Livermore, USA) which are integrated in the Algaemist system. In Fig. 4.1B, the emission spectra of the used light sources are shown. Reactor temperature was maintained at $37^{\circ} \mathrm{C}$, and the pH was maintained at $6.7( \pm 0.1)$ by means of on-demand $\mathrm{CO}_{2}$ supply. The reactors were operated in turbidostat mode to ensure a constant light regime; A light sensor was used to record the light passing through the reactor and if the outgoing light intensity dropped below the required setpoint (see below), the culture was automatically diluted with fresh medium employing a peristaltic pump. Further details of the photobioreactor setup and its operation are provided in (de Mooij et al. 2014), with the exception that the gas stream of di-nitrogen was, at all times, $500 \mathrm{~mL} \mathrm{~min}^{-1}$. The incident light intensity of the reactors was measured with a LI-COR 190-SA $2 \pi$ PAR (400-700 nm) quantum sensor (LICOR, USA) at 28 points evenly distributed over the light-exposed surface of the front glass panel of the culture chamber. A dummy reactor, with only a front glass panel installed, was used for the purpose of light measurements.

\subsubsection{Setup and operation of the short hydraulic retention time reactor}

The setup consists of three photobioreactors that were operated in turbidostat mode (See Fig. 4.2). In reactors 1 and 2 (high light reactors, illuminated from both sides) high light acclimated algae with reduced pigmentation were grown. The overflow of reactors 1 and 2 was connected to a third reactor (reactor 3 ). In the high light reactors 1 and 2 the specific growth rate $(\mu)$ was close to the maximum growth rate of this microalga. Because in steady state $\mu=D$, where $D$ is the dilution rate $\left(\mathrm{h}^{-1}\right)$, also reactor 3 was fed and diluted at a high rate. The result is that reactor 3 is characterized by a short hydraulic retention time (HRT), therefore, this reactor is referred to as the short HRT reactor. The high light reactors were illuminated from both sides by employing two LED panels. Cultures were exposed to $2500 \mu \mathrm{mol}$ photons $\mathrm{m}^{-2} \mathrm{~s}^{-1}(2 \times 1250 \mu \mathrm{mol}$ 
photons $\mathrm{m}^{-2} \mathrm{~s}^{-1}$ ). The outgoing light intensity on both sides was maintained at 20-25 $\mu \mathrm{mol}$ photons $\mathrm{m}^{-2} \mathrm{~s}^{-1}$. The short HRT reactor was operated under mass culture conditions comprising a solar noon sunlight intensity $\left(1500 \mu \mathrm{mol}\right.$ photons $\left.\mathrm{m}^{-2} \mathrm{~s}^{-1}\right)$ and a biomass concentration $\left(C_{x}\right)$ high enough to absorb more than $98 \%$ of the incoming light resulting in an outgoing light intensity of $10 \mu \mathrm{mol}$ photons $\mathrm{m}^{-2} \mathrm{~s}^{-1}$. This value is equal to the compensation point of photosynthesis for Chlorella sorokiniana according to our model estimation for cells with an absorption cross section as measured in a mass culture. Selecting the photosynthetic compensation point as the outgoing light intensity will effectuate maximal photobioreactor productivity (Takache et al. 2010). The biomass concentration in the high light reactors was slightly higher than the biomass concentration required in the short HRT reactor to obtain this outgoing light intensity of $10 \mu \mathrm{mol}$ photons $\mathrm{m}^{-2} \mathrm{~s}^{-1}$. Additional dilution of the short HRT reactor with fresh medium, therefore, was necessary and was applied automatically through the turbidostat control of the reactor.

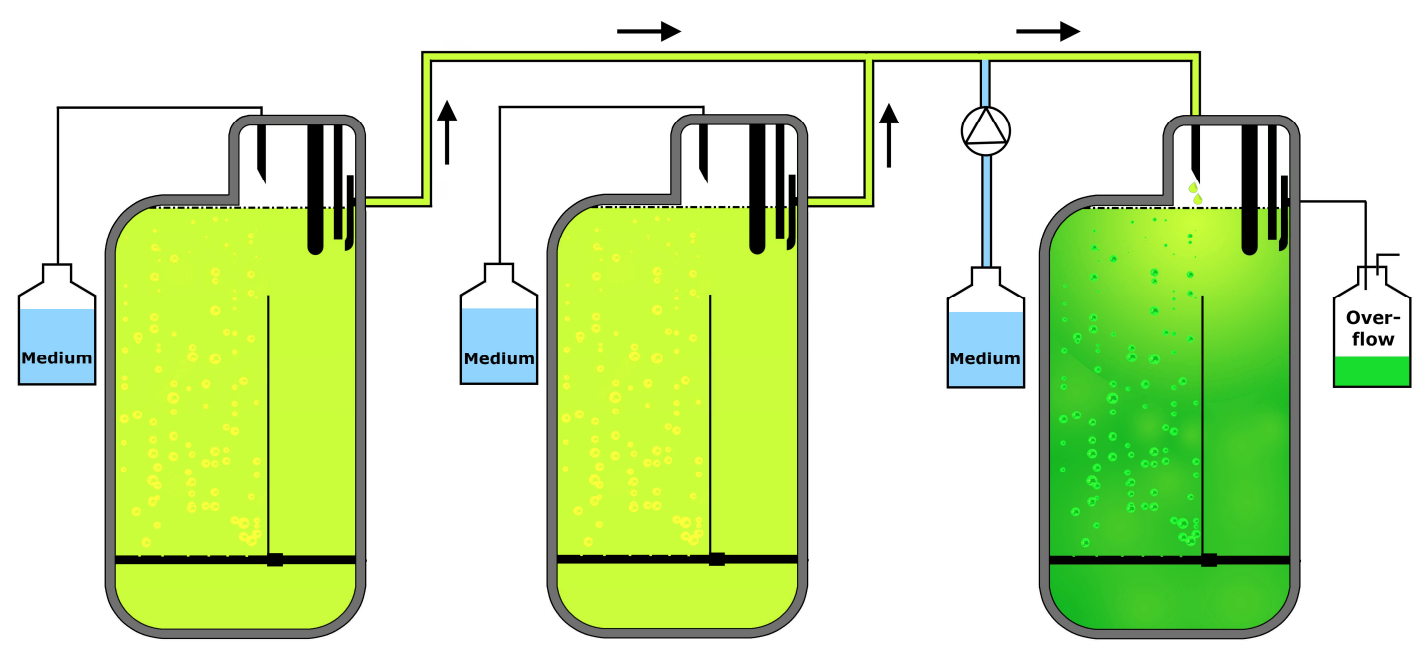

Fig. 4.2 Schematic overview of the experimental setup with two reactors containing high light (HL) acclimated algae, and a third reactor which was fed from the other two reactors and is therefore characterized by a short retention time (HRT). Each reactor was operated in turbidostat mode and was diluted on-demand with fresh medium. Arrows denote the direction of the flow of the microalgae suspension and the off-gas. For simplicity, the attached flasks are not drawn to scale. 
The high light cultures were first cultivated in batch. After the desired biomass concentration was achieved and turbidostat control was initiated, cultures were allowed to acclimate to the cultivation conditions for at least 36 hours. Subsequently, their overflow was coupled to the short HRT reactor. The biomass concentration and the diluted volume of the short HRT reactor were measured on a daily basis. It was ensured that the cultivation was stable during this period and that the biomass concentration and dilution rate were constant. Every 24 hours, the microalgae suspension harvested in the overflow vessel was weighed and utilized for further analyses. After sufficient measurements were performed for the short HRT reactor, the high light reactors were operated for four more days. During this period, the overflow of the high light reactors was collected separately to measure the biomass productivity of each reactor. The equation used for calculating the volumetric biomass productivity in the short HRT reactor can be derived from the biomass balance where it is assumed that all reactors are ideally mixed:

$$
\begin{aligned}
& \frac{d\left(M_{x, S H R T}\right)}{d t}=D_{H L 1} \cdot C_{x, H L 1} \cdot V_{R, H L 1}+D_{H L 2} \cdot C_{x, H L 2} \cdot V_{R, H L 2}+\mu \cdot C_{x, S H R T} \cdot V_{R, S H R T}-D_{S H R T} \cdot C_{x, S H R T} \cdot V_{R, S H R T}(1) \\
& r_{x, v o l, H L}=D_{H L} \cdot C_{x, H L} \\
& r_{x, v o l, S H R T}=\mu \cdot C_{x, S H R T}
\end{aligned}
$$

where the subscripts $S H R T, H L 1$ and HL2 refer to the short HRT reactor and the two high light (HL) reactors. $M_{X, S H R T}$ is the biomass dry weight (g) in the short HRT reactor; $D$ is the dilution rate $\left(\mathrm{d}^{-1}\right) ; C_{X, S H R T}$ is the biomass dry weight concentration in the short HRT reactor; $V_{R}$ is the reactor volume (L); and $r_{x, v o l}$ is the volumetric biomass productivity $\left(\mathrm{g} \mathrm{L}^{-1} \mathrm{~d}^{-1}\right)$. By assuming no biomass accumulation $\left(d M_{x, S H R T} / d t=0\right)$, equation 4 is formulated:

$0=r_{x, v o l, H L 1} \cdot V_{R, H L 1}+r_{x, v o l, H L 2} \cdot V_{R, H L 2}+r_{x, v o l, S H R T} \cdot V_{R, S H R T}-D_{S H R T} \cdot C_{x, S H R T} \cdot V_{R, S H R T}$

Equation 4 can be simplified as follows:

$r_{x, v o l, S H R T}=D \cdot C_{x, S H R T}-r_{x, v o l, H L 1} \cdot \frac{V_{R, H L 1}}{V_{R, S H R T}}-r_{x, v o l, H L 2} \cdot \frac{V_{R, H L 2}}{V_{R, S H R T}}$ 
In our experiments, $r_{x, v o l, H L 1}$ and $r_{x, v o l, H L 2}$ could not be continuously measured during the short HRT experiment because the total amount of overflow was directed to the short HRT reactor. Instead, only $15 \mathrm{~mL}$ of overflow was harvested daily per high light reactor to estimate $r_{x, v o l, H L}$. This might not be representative for a $24 \mathrm{~h}$ sample and therefore it was decided to measure the productivity of the high light reactors on four consecutive days after the short HRT reactor was uncoupled. During this period, the total amount of overflow could be harvested during 24 hours which resulted in a more accurate assessment of the daily productivity of the high light reactors. Photobioreactor productivity is most effectively evaluated by comparing the areal biomass productivity (in $\mathrm{g} \mathrm{m}^{-2} \mathrm{~d}^{-1}$ ) in which the area reflects the illuminated area of the photobioreactor $\left(A_{R}\right)$ and, accordingly:

$r_{x, S H R T}=\frac{r_{x, v o l, S H R T} \cdot V_{R, S H R T}}{A_{R}}$

The biomass yield on light energy was calculated using the following equations:

$Y_{x / p h}=\frac{r_{x, S H R T}}{r_{p h}}$

$r_{p h}=\left(I_{p h, \text { in }}-I_{p h, o u t}\right) \cdot 1 \cdot 10^{-6} \cdot 86400 \frac{s}{d}$

Where $r_{p h}$ is the areal light absorption rate $\left(\operatorname{mol}_{\mathrm{ph}} \mathrm{m}^{-2} \mathrm{~d}^{-1}\right)$, and $I_{p h}$ is the ingoing and outgoing light intensity ( $\mu \mathrm{mol}$ photons $\mathrm{m}^{-2} \mathrm{~s}^{-1}$ ).

The hydraulic retention time (HRT) of the short HRT reactor can be calculated with:

$H R T=\frac{1}{D_{S H R T}}$

\subsubsection{Light-shift experiment}

A light-shift experiment was performed to study the kinetics of photoacclimation. The light intensity was decreased from an ingoing light intensity of $1000 \mu \mathrm{mol}$ photons $\mathrm{m}^{-2} \mathrm{~s}^{-1}$ and an outgoing light intensity of $450 \mu \mathrm{mol}$ photons $\mathrm{m}^{-2} \mathrm{~s}^{-1}$ to an ingoing light intensity of $60 \mu \mathrm{mol}$ photons $\mathrm{m}^{-2} \mathrm{~s}^{-1}$ and an outgoing light intensity of $6 \mu \mathrm{mol}$ photons $\mathrm{m}^{-2} \mathrm{~s}^{-1}$. The reactor was operated in turbidostat mode. After three days of cultivation under high light conditions and after it was ensured that the cultivation was stable, i.e., 
the biomass concentration, absorption cross section, and dilution rate were constant, the downshift in light intensity was applied. In the hours following the light regime shift, samples withdrawn from the reactor were analyzed for the absorption cross section.

\subsubsection{Biological oxygen monitor experimental setup}

The setup consists of two photobioreactors that were operated in turbidostat mode and a biological oxygen monitor. Samples taken from the two reactors were directly analyzed for their photosynthetic activity. The first reactor was operated under high light conditions and was illuminated from both sides employing one LED panel and an additional LED light source (warm white 45mil chip, Bridgelux, Livermore, CA) on the backside of the reactor. This high light culture was exposed to $1650 \mu \mathrm{mol}$ photon $\mathrm{m}^{-2} \mathrm{~S}$ ${ }^{1}\left(1 \times 1000+1 \times 650 \mu \mathrm{mol}\right.$ photons $\left.\mathrm{m}^{-2} \mathrm{~s}^{-1}\right)$. The outgoing light intensities were 60 and $40 \mu \mathrm{mol}$ photons $\mathrm{m}^{-2} \mathrm{~s}^{-1}$, respectively. The second reactor was run under the earlier defined mass culture conditions: the ingoing light intensity was $1500 \mu \mathrm{mol}$ photons m${ }^{2} \mathrm{~s}^{-1}$ and the outgoing light intensity was $10 \mu \mathrm{mol}$ photons $\mathrm{m}^{-2} \mathrm{~s}^{-1}$.

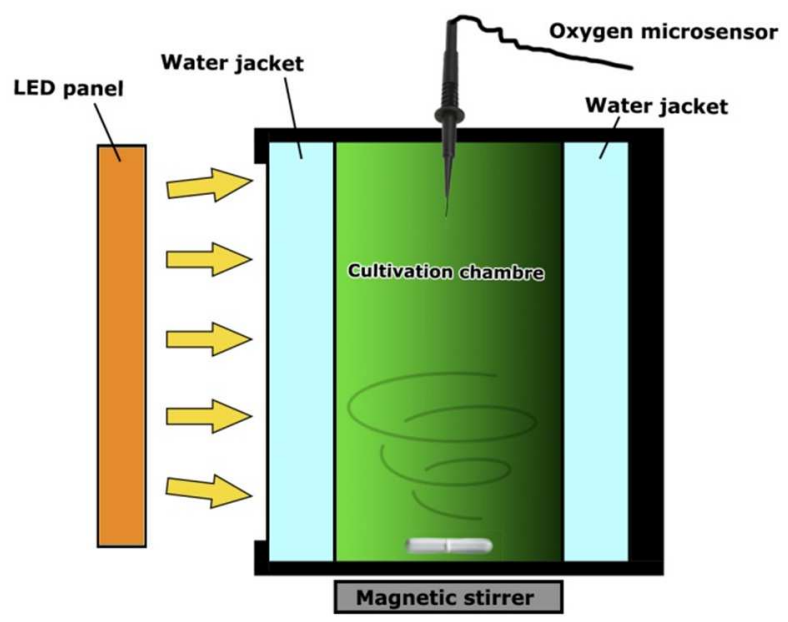

Fig. 4.3 Schematic overview of the biological oxygen monitor (BOM) setup.

Oxygen production by the microalgae was measured in a biological oxygen monitor (BOM) consisting of a Hansatech DW3 cultivation chamber (Hansatech instruments, Norfolk, UK) equipped with two water jackets for temperature control purposes (see Fig. 4.3). The cultivation chamber has a light path of $20 \mathrm{~mm}$ and a liquid volume of $15 \mathrm{~mL}$. The BOM was placed on a stirrer plate wherein a magnetic stirrer bar in the cultivation chamber provided mixing of the culture. The BOM was illuminated from one 
side (front optical window) by an Algaemist LED light panel (BXRA W1200, Bridgelux, Livermore, USA). The BOM was placed at a fixed position in front of the led panel. The incident light intensity behind the glass wall of the cuvette was calculated to be approximately $1600 \mu \mathrm{mol}$ photons $\mathrm{m}^{-2} \mathrm{~s}^{-1}$. Please refer to appendix B to see how the incident light intensity was calculated. The BOM incorporates a rear optical window that is normally covered by a cap, which allowed light transmission measurement with a LI-COR 190-SA $2 \pi$ PAR (400-700 nm) quantum sensor (LI-COR, USA). The temperature in the cultivation chamber was maintained at $37^{\circ} \mathrm{C}$ by water circulating through the water jackets and a temperature controlled water bath. The oxygen concentration in the chamber was measured using a fluorescence-based fiber-optic oxygen microsensor (IMP-PSt1-L5-LIC0-BGF3-TF-OIW, PreSens, Regensburg, Germany). The sensor tip (flat-broken, $\varnothing \approx 140 \mu \mathrm{m}$ ) was coated with an optical isolation. The oxygen-sensitive glass-fiber tip was sheltered inside a stainless steel syringe needle (40 $\mathrm{mm}$ length and $1.2 \mathrm{~mm}$ diameter) and was extended into the microalgae suspension only during measurements. The oxygen sensor was connected to a transmitter (Microx TX3, PreSens, Regensburg, Germany). Data collection was achieved through connecting the oxygen meter to a notebook and using the OxyView software (version 6.02, PreSense, Germany).

\subsubsection{Biological oxygen monitor operation}

A two-point calibration $(0 \%, 100 \%$ air-saturation) of the oxygen microsensor was performed on a daily basis prior to the measurements. The oxygen-free environment entailed calibration in a sodium sulfite solution sparged with nitrogen gas and the airsaturated environment entailed calibration in a water solution sparged with air. During calibration, the temperature was maintained at approximately $37^{\circ} \mathrm{C}$ and was measured with the external potentiometric temperature sensor (PT 1000) that is connected to the Microx TX3 transmitter for automatic temperature compensation. Inside the BOM cultivation chamber, M8-a medium ( $\mathrm{pH}$ 6.65) with a concentrated phosphorus buffer $\left(\mathrm{KH}_{2} \mathrm{PO}_{4}, 4.44 \mathrm{~g} \mathrm{~L}^{-1} ; \mathrm{Na}_{2} \mathrm{HPO}_{4} \cdot 2 \mathrm{H}_{2} \mathrm{O}, 1.56 \mathrm{~g} \mathrm{~L}^{-1}\right)$ was used to minimize $\mathrm{pH}$ perturbations.

At the moment that the cultivation in the photobioreactors was stable and productivity and biomass concentration were constant, microalgae cultures were transferred to the $\mathrm{BOM}$ in order to monitor oxygen accumulation under simulated mass culture 
conditions. To achieve an outgoing light intensity of $10 \mu \mathrm{mol}$ photons $\mathrm{m}^{-2} \mathrm{~s}^{-1}$, it was necessary to concentrate the samples from the high light reactor (centrifugation, $5 \mathrm{~min}$, $2500 \mathrm{rpm}$ and $20^{\circ} \mathrm{C}$ ) and dilute them with medium to obtain the appropriate biomass concentration. Resuspension of the pellets was done in M8-a medium ( $\mathrm{pH} \mathrm{6.65)} \mathrm{with} \mathrm{a}$ concentrated phosphorus buffer $\left(\mathrm{KH}_{2} \mathrm{PO}_{4}, 4.44 \mathrm{~g} \mathrm{~L}^{-1} ; \mathrm{Na}_{2} \mathrm{HPO}_{4} \cdot 2 \mathrm{H}_{2} \mathrm{O}, 1.56 \mathrm{~g} \mathrm{~L}^{-1}\right)$ in order to minimize $\mathrm{pH}$ perturbations during the measurements in the BOM. Samples from the mass culture (MC) photobioreactor had to be diluted because the light path of the BOM cuvette $(20 \mathrm{~mm})$ was longer than that of the reactor $(14 \mathrm{~mm})$. Dilution was done using M8-a medium with concentrated phosphorus buffer. MC samples were analyzed both with and without a centrifugation step to assess possible side effects of the centrifugation step. Prior to the sample injection into the BOM, each sample $(15 \mathrm{~mL})$ was sparged with pure nitrogen gas for three minutes to reduce the dissolved oxygen concentration in the algae suspension to $30 \% .100 \mu \mathrm{L}$ of a $0.3 \mathrm{M} \mathrm{NaHCO}$ solution was added just before sample injection in the BOM to prevent carbon limitation. For an additional three minutes, the microalgae were allowed to acclimate to low light intensities $\left(100 \mu \mathrm{mol}\right.$ photons $\left.\mathrm{m}^{-2} \mathrm{~s}^{-1}\right)$. The incident light intensity was then increased to $1500 \mu \mathrm{mol}$ photons $\mathrm{m}^{-2} \mathrm{~s}^{-1}$, and the oxygen concentration was measured for three minutes. After the high light measurement, the light was extinguished in order to monitor oxygen consumption under darkness for another three minutes.

The oxygen concentration was recorded in one-second intervals and expressed as $\mu \mathrm{mol}$ $\mathrm{O}_{2} \mathrm{~L}^{-1}$. The oxygen production rate $\left(\mu \mathrm{mol} \mathrm{O}^{2} \mathrm{~L}^{-1} \mathrm{~min}^{-1}\right)$ is equal to the slope of the dissolved oxygen concentration versus time. To calculate the slope, only values below $150 \%$ air-saturation were used. The biomass-specific oxygen production rate $\left(\mu \mathrm{mol} \mathrm{O}_{2}\right.$ $\mathrm{g}^{-1} \mathrm{~min}^{-1}$ ) was obtained by dividing the oxygen production rate by the biomass concentration. The biomass concentration of each sample injected into the BOM was measured by utilizing a separate dry-weight analysis.

\subsubsection{Analyses}

\section{Biomass dry weight concentration}

To determine the biomass dry weight content, the culture broth was passed through glass fiber filters as described by Kliphuis et al. (2012) and the mass difference 
between the dried empty filters and the dried filters with microalgae was subsequently recorded. All measurements on an individual sample were performed in triplicate.

\section{Absorption cross section}

Light absorption was measured in a double beam spectrophotometer (UV-2600, Shimadzu, Japan) equipped with an integrating sphere (ISR-2600). A reactor sample was transferred to a cuvette (100.099-OS, Hellma, Germany) with a short light path of 2 $\mathrm{mm}$. The same reactor sample was analyzed for its dry weight content. This allowed normalization of the measured absorbance, resulting in an absorption cross section which is based on dry weight which carries the unit $\mathrm{m}^{2} \mathrm{~g}^{-1}$. Additional details of this protocol have been described by (de Mooij et al. 2014). In this study, samples were not diluted prior to measurements as the biomass concentration was still in the linear range of the absorption measurement.

\section{Maximum photosystem II quantum yield}

The maximum photosystem II (PSII) quantum yield $\left(F_{v} / F_{m}\right)$ was measured from samples withdrawn directly from the reactor. Samples were diluted to obtain a biomass density of approximately $25 \mathrm{mg} \mathrm{L}^{-1}$. Following dark adaption for ten minutes, fluorescence of the microalgae samples was measured with a technique based on pulse amplitude modulated (PAM) fluorometry (AquaPen-C AP-C 100, PSI, Czech Republic). According to the manufacturer's manual, the actinic light intensity should be $270 \mu \mathrm{mol}$ photons $\mathrm{m}^{-2} \mathrm{~s}^{-1}$; the saturating light intensity should be $2430 \mu \mathrm{mol}$ photons $\mathrm{m}^{-2} \mathrm{~s}^{-1}$; and the measuring light intensity should be $0.03 \mu \mathrm{mol}$ photons $\mathrm{m}^{-2} \mathrm{~s}^{-1}$. The excitation light wavelength was $620 \mathrm{~nm} . F_{v} / F_{m}$ is the ratio of variable fluorescence to maximal fluorescence (Eq. 10). In this equation, $F_{o}$ is the dark-adapted minimum level of fluorescence. $F_{m}$ is the maximum level of fluorescence measured following a short light pulse of high intensity. $F_{v}$ (variable fluorescence) is defined as the difference between $F_{m}$ and $F_{o}$, and represents the difference between the fluorescence intensities with closed and open PSII reaction centers.

$F_{v} / F_{m}=\left(F_{m}-F_{0}\right) / F_{m}$ 


\subsection{Results and discussion}

\subsubsection{Pigment accumulation rate following a downshift in light intensity}

To obtain and maintain microalgae with a reduced pigment content, knowledge regarding the pigment accumulation kinetics is of major importance. We performed a light shift-down experiment in which the light was shifted down from an ingoing light intensity of $1000 \mu \mathrm{mol}$ photons $\mathrm{m}^{-2} \mathrm{~s}^{-1}$ and an outgoing light intensity of $450 \mu \mathrm{mol}$ photons $\mathrm{m}^{-2} \mathrm{~s}^{-1}$ to an ingoing light intensity of $60 \mu \mathrm{mol}$ photons $\mathrm{m}^{-2} \mathrm{~s}^{-1}$ and an outgoing light intensity of $6 \mu \mathrm{mol}$ photons $\mathrm{m}^{-2} \mathrm{~s}^{-1}$. Under these conditions light is severely limiting and growth is expected to be slow.
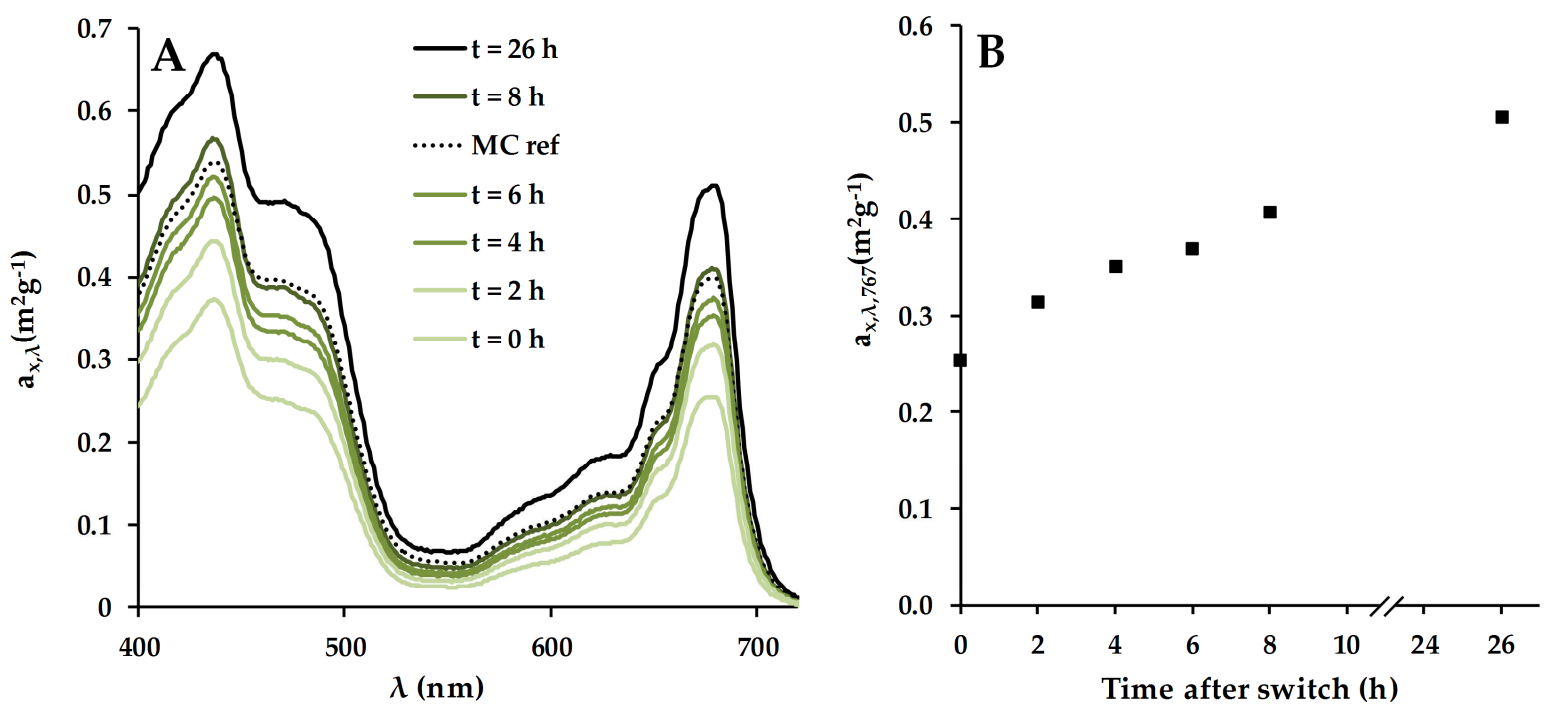

Fig. 4.4 (A) Absorption cross section $\left(a_{x, \lambda}\right)$ increase of Chlorella sorokiniana following a light down shift. The absorption cross section has been normalized to the measured biomass concentration in the reactor. Lines represent single measurements. Time $t=0$ min represents the moment of the down shift. (B) Absorption cross section $\left(a_{x, 2,667}\right)$ increase of $C$. sorokiniana following the light down shift.

In Fig. 4.4 the increase of the absorption cross section is presented. The darker conditions after the light down shift led to the highest measured absorption cross section ( $\mathrm{t}=26$ h, Fig. 4.4), which was higher than obtained in a mass culture. As can be seen in Fig 4.4B, the rate at which the absorption cross section in the reactor increases follows a linear trend in the first eight hours of acclimation. What becomes evident in this acclimation experiment is that, under these relative dark conditions, it takes six to 
eight hours to reach the absorption cross section that is normally observed in a mass culture (MC ref. in Fig. 4.4A), and that it takes even longer to obtain the maximum value for the absorption cross section.

\subsubsection{Model estimation of biomass yield on light energy for different absorption cross sections and light gradients}

The light use efficiency that can be expected by decreasing the absorption cross section within the genetic boundaries of $C$. sorokiniana, and the effect of various light regimes, was estimated by employing a microalgae growth model in combination with a mathematical description of the light gradient in a microalgae culture in a photobioreactor. A description of the model is reported in appendix A. The biomass yield on light energy $\left(Y_{x / p h}\right)$ and areal productivity $\left(r_{x}\right)$ are presented in Fig. 4.5A. MC is a normal mass culture (experimental data presented in section 4.3.3). MC LP is a mass culture with a low pigmentation as obtained in the short HRT experiment, when high light (HL) acclimated cells are retained in a normal mass culture for 2 hours (experimental data presented in section 4.3.3). MC HL accl. is a hypothetical mass culture with the pigmentation of a HL culture. HL accl is a HL acclimated culture exposed to double sided illumination (experimental data presented in section 3.4). All absorption cross sections that were used as input for the model calculations (see Fig. 4.6) originate from the actual experiments described in this paper. What is clearly evident from Fig. 4.5A is that, based on the model, an apparent increase in biomass productivity would be expected upon a reduction in the absorption cross section. 

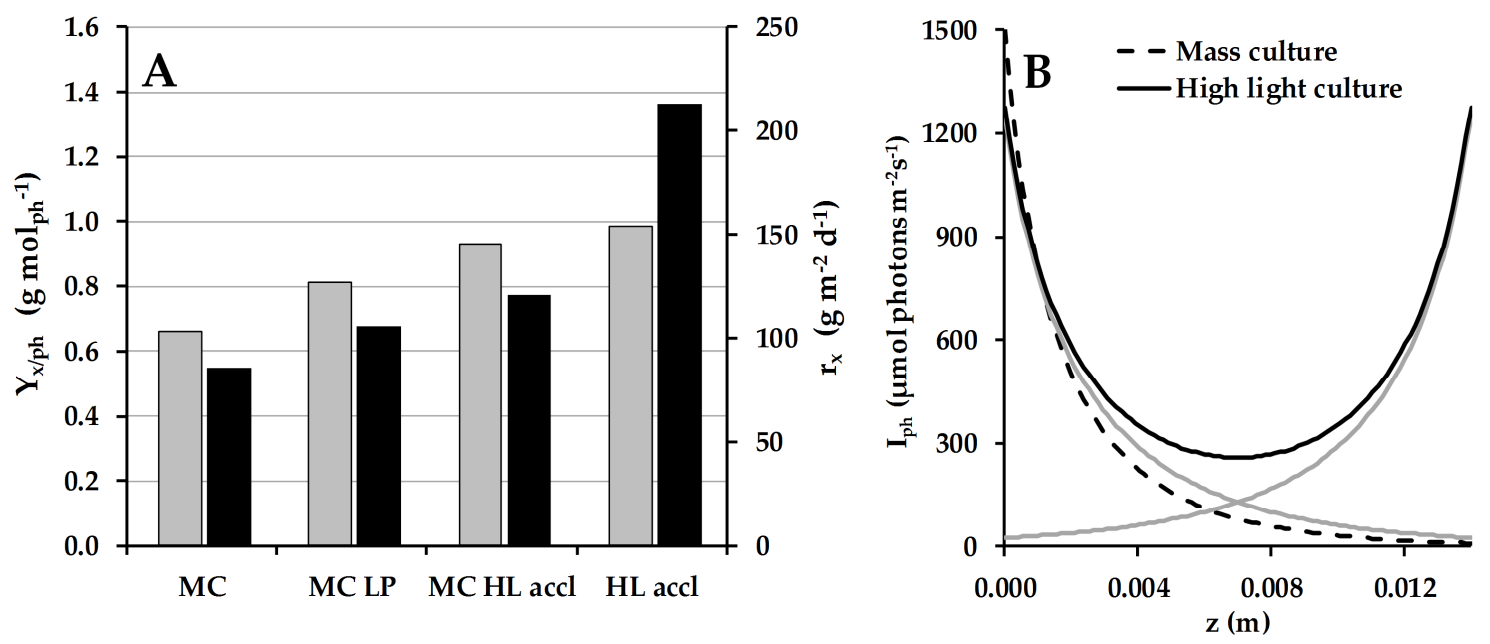

Fig. 4.5 (A) Model estimation of the biomass yield on light energy ( $Y_{x / p h}$ grey bars) and the areal biomass productivity ( $r_{x}$, black bars) that can be attained under different conditions: a normal mass culture (MC), a mass culture with a low pigment content (MC LP, as obtained in the short HRT experiment), a hypothetical mass culture with the pigmentation of a high light exposed culture (MC HL accl), and a high light culture under double-sided illuminated high light conditions (HL accl). For the mass cultures, the ingoing light intensity was $1500 \mu \mathrm{mol}$ photons $\mathrm{m}^{-2} \mathrm{~s}^{-1}$ and the outgoing light intensity was $10 \mu \mathrm{mol}$ photons $\mathrm{m}^{-2} \mathrm{~s}^{-1}$. For the high light culture, it was $2 \times 1250 \mu \mathrm{mol}$ photons $\mathrm{m}^{-2} \mathrm{~s}^{-1}$ ingoing and $2 \times 25 \mu \mathrm{mol}$ photons $\mathrm{m}^{-2} \mathrm{~s}^{-1}$ outgoing. (B) Light intensity gradient for the mass culture and the high light culture. The grey lines exhibit the light gradient as generated by the individual light sources of the double-sided illuminated culture while the black solid line is the sum of those gradients.

The highest yield is obtained under high light conditions with double-sided illumination and as the total amount of light supplied and absorbed was much higher, also the areal biomass productivity was higher than for the other model estimations. There are two reasons for the higher biomass yield on light. First, although the average light intensity is considerably higher than that of the mass cultures, the peak light intensity was only $1250 \mu \mathrm{mol}$ photons $\mathrm{m}^{-2} \mathrm{~s}^{-1}$ which results in less dissipation than the $1500 \mu \mathrm{mol}$ photons $\mathrm{m}^{-2} \mathrm{~s}^{-1}$ of the mass cultures (Fig. 4.5B ). Second, as a high specific growth rate occurs at every position in the reactor, the negative contribution of the maintenance costs to the overall productivity is minimal. This also signifies that there is a larger volume fraction of the reactor where the light intensity is close to the optimal value. Summarized, it can be concluded that less light absorption increases light use efficiency but above all that a more homogeneous light distribution in the reactor increases the biomass yield on light. 


\subsubsection{Absorption cross section and biomass productivity of a high light acclimated culture under mass culture conditions}

In Fig. 4.6, the absorption cross section $\left(a_{x, \lambda}\right)$ is shown for three different conditions: high light conditions, mass culture conditions, and mass culture conditions fed with high light acclimated cells at a short HRT of two hours. If we consider the absorption cross section of the mass culture to be $100 \%$, then $59 \%$ of the absorption cross section remains under high light conditions as the consequence of photoacclimation. When exposed to the mass culture conditions with an average retention time of two hours, $76 \%$ of the mass culture absorption cross section was observed. This indicates that, the pigment accumulation process begins to increase the cellular light absorption capacity directly after entering the mass culture. The ratio of light absorption at the wavelengths $675 \mathrm{~nm}$ and $440 \mathrm{~nm}$ did not significantly vary for the three conditions, indicating that there was no change in the carotenoid content in comparison to chlorophyll a. The biomass concentration $\left(C_{X}\right)$ in a normal mass culture was consistently $2.3 \pm 0.2 \mathrm{~g} \mathrm{~L}^{-1}$ versus $3.0 \pm 0.1 \mathrm{~g} \mathrm{~L}^{-1}$ for the culture in the short HRT reactor, which confirms the higher transparency of the cells since turbidostatic operation ensured that the same amount of light was absorbed.

The absorption cross section of culture in the reactor with a two hours HRT was higher than expected compared to the light shift experiment that was performed, where it took at least six hours before the culture was acclimated. A possible explanation is that the light shift experiment was executed under relatively dark conditions and therefore the cells may not have received sufficient light energy for a rapid increase in pigmentation. The severe light limitation could have resulted in a slower increase in absorption cross section than the rate that was observed in the short HRT experiment. The short HRT experiment was also performed with a retention time of six hours (data not shown), and no difference in the absorption cross section was observed in comparison with a normal mass culture. 


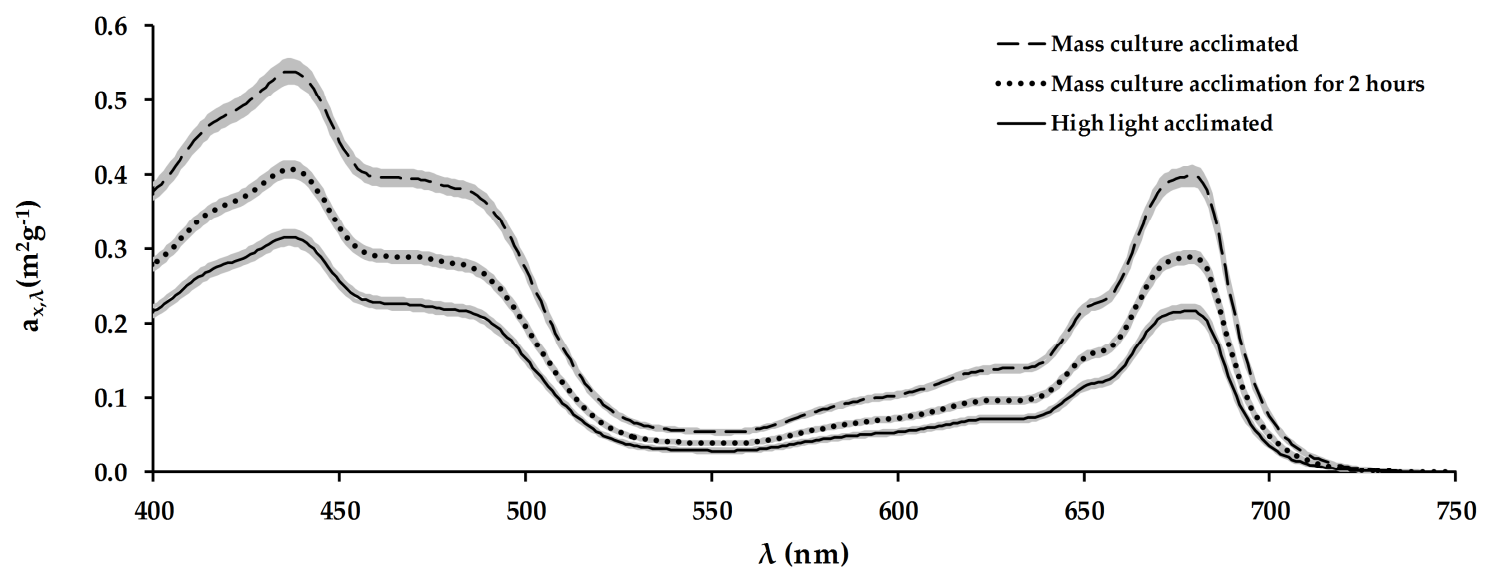

Fig. 4.6 Absorption cross section ( $a_{x, \lambda}$ ) of Chlorella sorokiniana under three different conditions. The absorption cross section has been normalized to the biomass dry weight. Lines represent the average \pm $S D$ of six measurements for the fully acclimated mass culture, 19 measurements for the two hours acclimated mass culture, and 58 measurements for the high light acclimated culture. All measurements originate from two separate experiments. As previously explained, one experiment consists of one mass culture reactor and two high light reactors.

The areal biomass productivity of a normal mass culture ranged between 99 and $119 \mathrm{~g}$ $\mathrm{m}^{-2} \mathrm{~d}^{-1}$ (Fig. 4.7). For the short HRT reactor, in one experiment, we achieved a productivity similar $\left(107 \mathrm{~g} \mathrm{~m}^{-2} \mathrm{~d}^{-1}\right)$ to that observed for normal mass cultures and, for the other experiment, productivity was considerably lower $\left(81 \mathrm{~g} \mathrm{~m}^{-2} \mathrm{~d}^{-1}\right)$. As previously explained in the materials and methods section, it was not possible to measure the productivity of the high light reactors in the same interval as the short HRT reactor. Although there were no indications that the obtained steady states were disturbed, this may have caused a more substantial experimental error than normally observed in mass cultures. To conclude, the decreased pigmentation in the short HRT reactor did not lead to increased productivity. This is in contrast with our model estimations, which suggested that the productivity could be 1.2 times higher compared to a normal mass culture. Possible explanations for this unexpected result will be discussed later.

The average of all $\mathrm{F}_{\mathrm{v}} / \mathrm{F}_{\mathrm{m}}$ values for the normal mass cultures was 0.72 ; and individual $F_{v} / F_{m}$ values did not decrease below 0.69 . The average $F_{v} / F_{m}$ value of all short HRT reactor measurements was 0.71 and that of the high light reactors was 0.69 . The $F_{v} / F_{m}$ parameter represents the maximum photosystem II (PSII) quantum yield measured after dark adaptation. Reduced $\mathrm{F}_{\mathrm{v}} / \mathrm{F}_{\mathrm{m}}$ values indicate impaired PSII function which can be caused by strong oversaturation (Björkman et al. 1987). According to our experience with $C$. sorokiniana, $\mathrm{F}_{\mathrm{v}} / \mathrm{F}_{\mathrm{m}}$ values exceeding 0.70 indicate optimal PSII 
performance. For the precise values of light intensities and the obtained experimental data of each experiment, please refer to Appendix E and Appendix F.

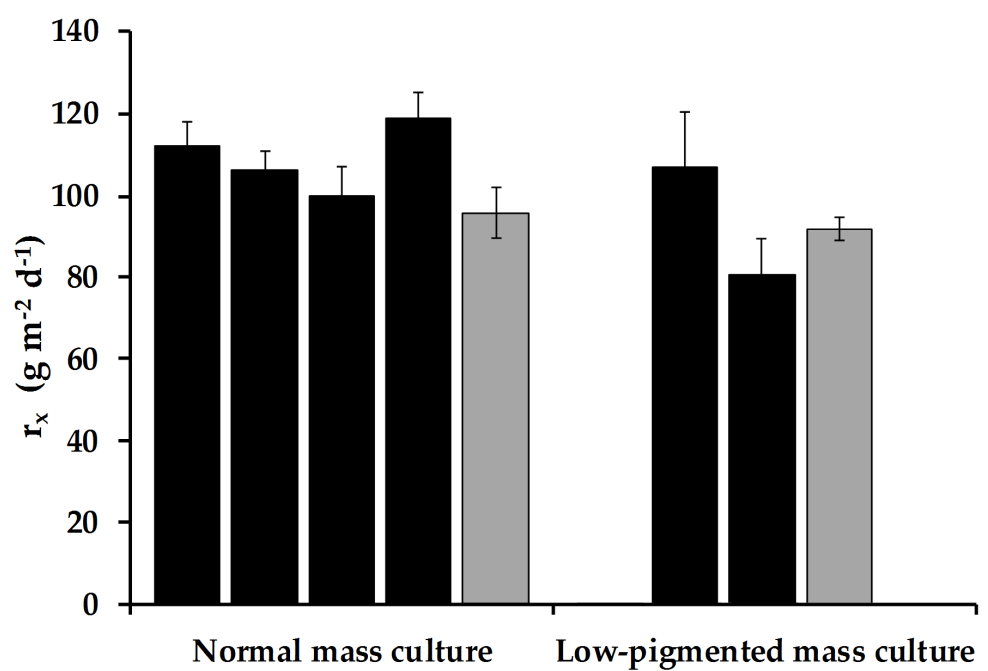

Fig. 4.7 Areal biomass productivity $\left(r_{x}\right)$ of normal mass cultures and mass cultures containing high light acclimated cells that possess a decreased absorption cross section. Each black bar represents the average $\pm S D$ of $n \geq 4$ measurements during a separate reactor experiment, where each measurement corresponds to the productivity over a single day. The grey bars are oxygen production rates measured in the biological oxygen monitor (BOM) that were converted to areal biomass productivity. The incident light intensity in the BOM experiment was slightly higher ( $\pm 1600 \mu \mathrm{mol}$ photons $\left.\mathrm{m}^{-2} \mathrm{~s}^{-1}\right)$ than the other mass cultures $\left( \pm 1500 \mu \mathrm{mol}\right.$ photons $\mathrm{m}^{-2} \mathrm{~s}^{-1}$ ). The average $\pm S D$ of the BOM-based productivity represents $\geq 6$ measurements performed within one reactor experiment (1 measurement per day).

\subsubsection{Biomass yield on light energy of high light acclimated cells in a high light regime}

The performance of the high light acclimated cells was studied to investigate the effect of various light regimes on the light use efficiency. Results are presented in terms of biomass yield on light energy $\left(Y_{x / p h}, \mathrm{~g} \mathrm{~mol}_{\mathrm{ph}^{-1}}\right)$ to afford a better comparison since different amounts of light energy were absorbed under the various light regimes. The $Y_{x / p h}$ is depicted in Fig. 4.8 for the mass cultures that were previously discussed, and for four cultures that were grown under high light conditions. As is evident from Fig. 4.8, the $Y_{x / p h}$ of high light acclimated cultures $\left(1.02 \mathrm{~g} \mathrm{~mol}_{\mathrm{ph}}{ }^{-1}\right)$ was, higher than that of the mass cultures $\left(0.86 \mathrm{~g} \mathrm{~mol}_{\mathrm{ph}^{-1}}\right)$. Average $\mathrm{F}_{\mathrm{v}} / \mathrm{F}_{\mathrm{m}}$ values of all cultures were 0.70 or higher, indicating that the light regime did not severely damage the photosystems. 


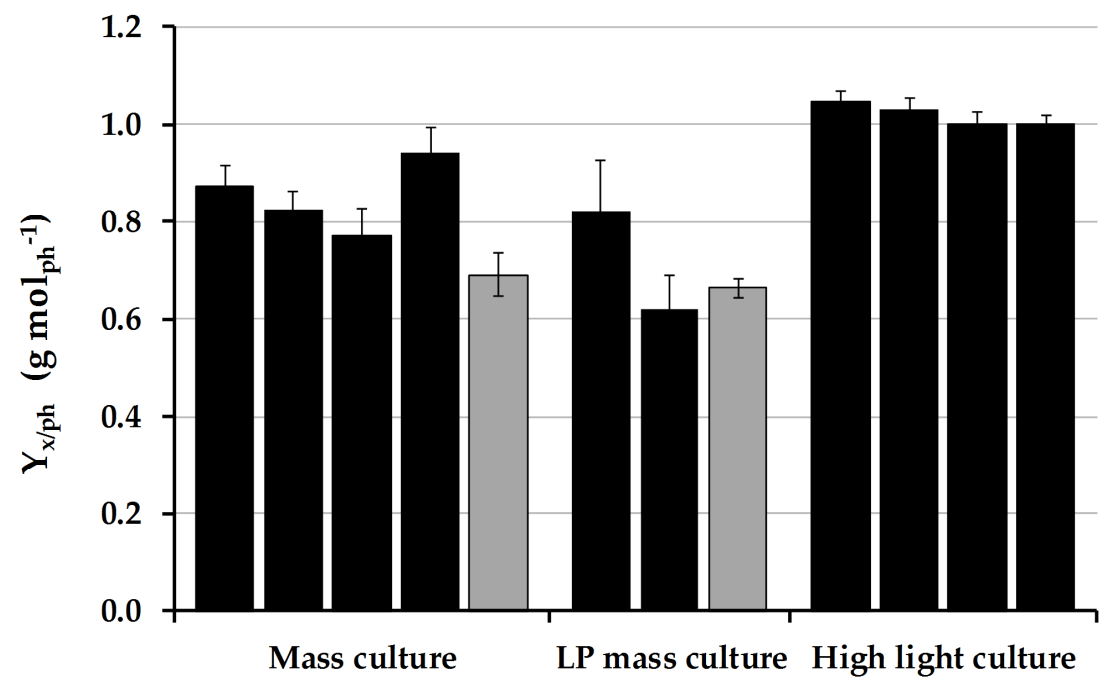

Fig. 4.8 Biomass yield on light energy $\left(Y_{x / p h}\right)$ of normal mass cultures, low-pigmented (LP) mass cultures, and high light exposed cultures in double sided illuminated reactors. Each black bar represents the average $\pm S D$ of $n \geq 4$ measurements during a separate reactor experiment, where each measurement corresponds to the productivity over a single day. All mass cultures represented by the solid black bars were illuminated at $1500 \mu \mathrm{mol}$ photons $\mathrm{m}^{-2} \mathrm{~s}^{-1}$ with an outgoing light intensity of 10 $\mu \mathrm{mol}$ photons $\mathrm{m}^{-2} \mathrm{~s}^{-1}$. The high light cultures were illuminated from two sides at $2 \times 1250 \mu \mathrm{mol}$ photons $\mathrm{m}^{-2} \mathrm{~s}^{-1}$ with outgoing light intensities of $2 \times 25 \mu \mathrm{mol}$ photons $\mathrm{m}^{-2} \mathrm{~s}^{-1}$. The grey bars represent converted oxygen production rates measured in the BOM under mass culture conditions. The incident light intensity in this experiment was moderately higher ( $\pm 1600 \mu \mathrm{mol}$ photons $\left.\mathrm{m}^{-2} \mathrm{~s}^{-1}\right)$ than the other mass cultures.

The purpose of our model predictions (section 3.2) was to calculate the relative gain in light use efficiency that can be obtained by reduction of the absorption cross section. The model well estimated the productivity of the high light cultures that had a more homogenous light regime. However, the model underestimated the biomass productivity of normal mass cultures when compared to our experimental dataset. Even though all of our input parameters are based on the most optimistic estimates that could be ascertained from literature, the measured productivity in our reactor experiments was 15-30\% higher than predicted. Apparently C. sorokiniana can perform photosynthesis at a higher rate than expected based on the model calculations employing literature estimates of its biological kinetic constants and yield parameters. Also Blanken et al. found that under similar mass culture conditions growth of $C$. sorokiniana is underestimated based on literature derived biological parameters (Blanken et al. 2016). 


\subsubsection{Oxygen production rate of a high light acclimated mass culture in a biological oxygen monitor}

Two independent photobioreactors were operated: one under typical mass culture conditions, and the other under a high light regime with double-sided illumination with an ingoing light intensity of $1 \times 1000$ and $1 \times 650 \mu \mathrm{mol}$ photons $\mathrm{m}^{-2} \mathrm{~s}^{-1}$ and an outgoing light intensity of $\pm 50 \mu \mathrm{mol}$ photons $\mathrm{m}^{-2} \mathrm{~s}^{-1}$. The resulting absorption cross section is depicted in Fig. 4.9B. The pigmentation in the high light culture was considerably reduced compared to the mass culture, but did not reach minimum values as we have measured lower absorption cross sections before (see Fig. 4.6). Microalgae cultures withdrawn from these reactors were analyzed in a biological oxygen monitor (BOM) using short term oxygen production measurements. The BOM was illuminated with $\pm 1600 \mu \mathrm{mol}$ photons $\mathrm{m}^{-2} \mathrm{~s}^{-1}$, and the biomass concentrations of the samples were diluted such that the outgoing light intensity was approximately $10 \mu \mathrm{mol}$ photons $\mathrm{m}^{-2} \mathrm{~s}^{-1}$. Due to the lower absorption cross section of the high light acclimated cells, a 1.5 times higher biomass density was required in the BOM to absorb all of the incident light.

As presented in Fig. 4.9A, the volumetric oxygen production rate (OPR) of the high light acclimated cells $\left(148 \pm 5 \mu \mathrm{mol} \mathrm{O} \mathrm{L}^{-1} \mathrm{~min}^{-1}\right)$ was not higher than the OPR obtained for the mass culture acclimated cells (average: $154 \pm 10 \mu \mathrm{mol} \mathrm{O} \mathrm{L}^{-1} \mathrm{~min}^{-1}$ ). The oxygen consumption rate (OCR) was measured under darkness in a three minute period after exposure to $1600 \mu \mathrm{mol}$ photons $\mathrm{m}^{-2} \mathrm{~s}^{-1}$. This OCR is considered to reflect respiratory oxygen consumption in the light (Kliphuis et al. 2011) and was highest for the high light acclimated cells. Taking into account the 1.5 times higher biomass concentration of the high light acclimated cells in the BOM, it can be concluded that the high light acclimated cells absorbed less light per unit of biomass, and less oxygen was produced per unit of biomass compared to the cells acclimated to mass culture conditions. The amount of oxygen consumed per unit of biomass directly after the measurements was equal in both situations. Kliphuis et al. (2011) found biomass specific OCR values that are in accordance (25-33 $\mu \mathrm{mol} \mathrm{O} \mathrm{min}^{-1} \mathrm{~g}^{-1}$ ) with our measurements (29 and $31 \mu \mathrm{mol} \mathrm{O}_{2} \mathrm{~min}^{-}$ ${ }^{1} \mathrm{~g}^{-1}$ for the mass culture and the high light culture, respectively). 

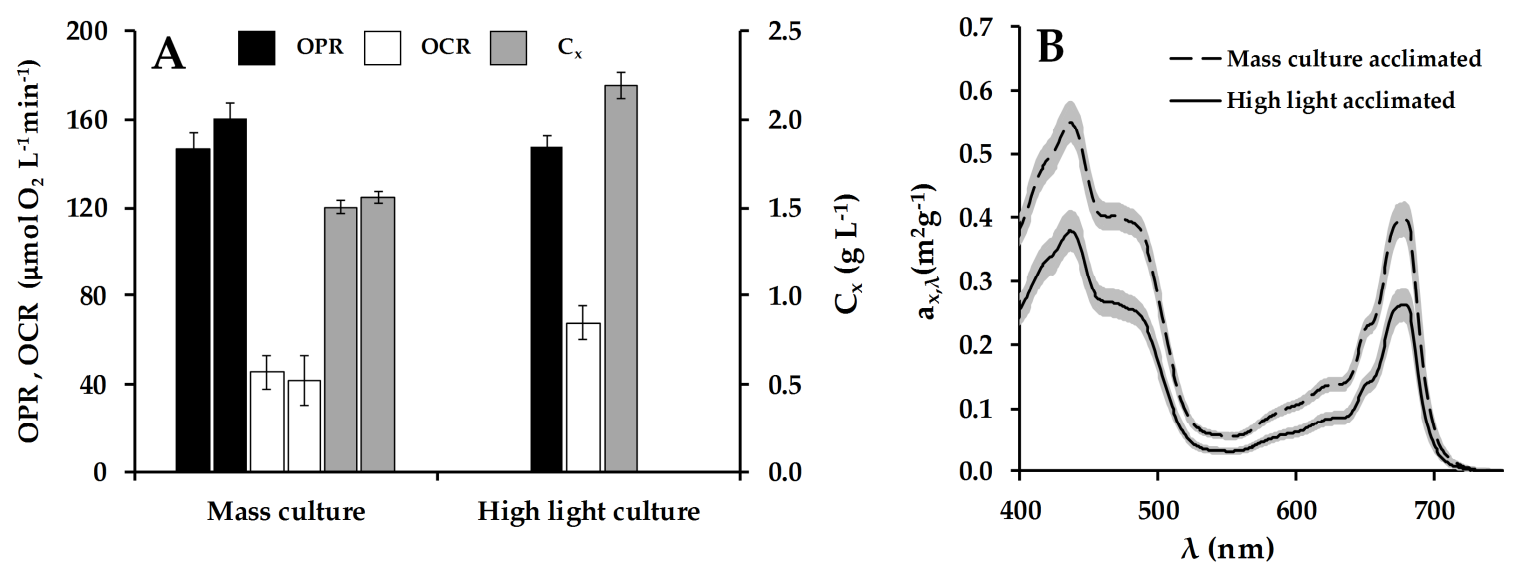

Fig. 4.9 (A) Oxygen production rate (OPR), Oxygen consumption rate (OCR) and biomass concentration $\left(C_{x}\right)$ of $C$. sorokiniana as measured in the biological oxygen monitor (BOM). Prior to the measurements, cells were acclimated to either high light conditions or mass culture conditions for several days in flat panel photobioreactors. Each bar represents the average $\pm S D$ of at least seven data points that were measured within at least five consecutive days. The bars of the mass culture represent duplicate experiments, performed with microalgae originating from the same photobioreactor. (B) Absorption cross section $\left(a_{x, \lambda}\right)$ of the cells for the two different acclimation states. Lines represent the average $\pm S D$ of at least four measurements.

The fact that the biomass specific OCR for mass culture cells and high light acclimated cells were similar would normally indicate that the cells have the same metabolic activity as the respiration rate can be directly related to the growth rate (Falkowski et al. 1985). At higher growth rates, more metabolic energy is required in the form ATP which leads to an increased respiration and thus increased consumption of oxygen (Kliphuis et al. 2012). It would, therefore, be expected that because of the higher biomass concentration in the high light acclimated culture with the same metabolic activity, also the OPR would be higher. This was not the case and the high light acclimated cells apparently have a higher respiration rate relative to the specific growth rate, indicating that other processes apart from growth occur that consume oxygen. These processes could be related to the metabolic costs of the photoacclimation process itself or other processes that can be attributed to the cells being acclimated to high light conditions.

The high light acclimated samples were concentrated by means of centrifugation to obtain a biomass concentration high enough to absorb all incoming light in the BOM. To exclude undesirable side effects of centrifugation, the mass culture samples were analyzed twice in the BOM: with and without a centrifugation step. No significant difference was observed between these treatments, therefore, the results are depicted 
as duplicate experiments in Fig. 4.9A. To compare the OPR to the productivity values from other experiments, the OPR values were converted to areal productivity and presented in Fig. 4.8. For the details of this conversion, please refer to appendix C. The conversion resulted in productivity values and biomass yield on light energy comparable to the lowest one measured in the previous reactor experiments (Fig. 4.8).

\subsubsection{Possible explanations for the discrepancy between the model estimations and the experimental results}

In both experimental setups, the short HRT reactor and the BOM, temporary reduction of the absorption cross section did not result in increased biomass productivity. This while our model estimated that a $23 \%$ higher productivity could be expected for lowpigmented cells in the short HRT reactor and a 30\% increase for the high light acclimated mass culture in the BOM. In our model estimations, it is assumed that the only difference between cells from a mass culture and cells from a high light acclimated culture is the absorption cross section. However, at least one other phenomenon must have been involved that has not yet been considered. Based on the available experimental data, we have no solid explanation for the lower-than-estimated biomass productivity of high light acclimated under mass culture conditions, though we have two possible explanations supported by literature that are discussed below.

We consider an increased light energy dissipation rate as a consequence of long term high light acclimation to be the most likely explanation for our results. This light energy dissipating phenotype, initiated for survival purposes, could have sustained in the mass culture and resulted in reduced light use efficiency. There are several energy dissipation mechanisms that act on different levels of organization (Wobbe et al. 2016). Non photochemical quenching (NPQ) is the most studied mechanism. It is known to consist of several components each with a different induction and relaxation time (Müller et al. 2001, Erickson et al. 2015). One of these components (qI) is described as a sustained thermal dissipation mechanism that is slowly reversible (range of hours) probably due to a combination of photoprotection and photodamage (Müller et al. 2001, Lambrev et al. 2010, Erickson et al. 2015). Reaching maximum dissipation capacity involves the biosynthesis of enzymes and pigments such as zeaxanthin. It is not unlikely that, once established, the greater NPQ capacity continues to be available 
for a certain period of time after the transfer of the algae to a mass culture. Also, according to Adams et al., in higher plants it has been determined that increases in thermal energy dissipation can, under certain conditions, be sustained after a switch from high light to low light conditions (Demmig-Adams et al. 1992).

Microalgae have been reported to radically increase their NPQ capacity even at moderate light intensities (Bonente et al. 2012, Cazzaniga et al. 2014), thereby reducing the photosynthetic efficiency. Although high light acclimated cells or leaves have a higher NPQ capacity, it remains ambiguous whether its activation occurs at the same light intensity as that for normal cells or leaves. It has been reported that high light acclimated cells are not stimulated as quickly as dark adapted cells (Sforza et al. 2015) which appears to be strain dependent. The fact that we measured high $F_{v} / F_{m}$ values for most experiments does not guarantee a high light use efficiency. It is only an indicator of adequate functioning of the PSII. The photosynthetic efficiency and productivity of the reactor can, in this case, still be affected by increased thermal energy dissipation (MacIntyre et al. 2002). In short, it seems plausible that once the thermal dissipation capacity has been increased after a period of high light acclimation, higher-than-normal dissipation rates remain active after a sudden switch to mass culture conditions.

Microalgae have been discovered to accumulate screening pigments under high light conditions. These extra-thylakoid pigments such as $\beta$-carotene do not transfer excitation energy to the reaction centers and thereby optically screen the cells and prevent oversaturation and damage to the light harvesting complexes (SiefermannHarms 1985, Solovchenko 2010). When formed during high light acclimation, they will probably not instantly disappear after a transfer to low light conditions and, therefore, may decrease photosynthetic efficiency (MacIntyre et al. 2002). Also, according to Falkowski et al., screening pigments are the reason that high light acclimated cells often have a reduced quantum yield (Falkowski et al. 2013). C. sorokiniana was found to contain several of these auxiliary pigments (de-Bashan et al. 2008, Cazzaniga et al. 2014), though in one study, it was shown that the total contribution of these screening pigments to total NPQ was limited (Masojídek et al. 1999). We did not measure a relative change in the absorption spectrum after a transfer from high light to mass culture. However, because accessory carotenoids in principle could be replaced by 
screening carotenoids and absorb light at the same wavelength range, such screening pigments could be difficult to distinguish (MacIntyre et al. 2002).

The other explanation for the observed loss in light use efficiency is that, a significant amount of energy is expended on the photoacclimation process itself, and this could counterbalance the theoretical gain in light use efficiency that can be obtained. The physiological transformation in cells upon a shift from high light to low light conditions entails de novo biosynthesis of pigments and protein complexes, a major ultrastructural reorganization, transcriptional alterations, and changes in the composition of the LHC (Dubinsky et al. 2009). If the metabolic activity is, indeed, completely focused on photoacclimation, we cannot consider the culture to be in a state of 'balanced growth' that is representative for normal growth conditions (MacIntyre et al. 2002), and this may have reduced the measured productivity. In a recent study it was found that cyanobacteria capable of adjusting their phycobilisome content depending on the light intensity suffered from a short-term growth impairment compared to cyanobacteria without phycobilisomes (Agostoni et al. 2016). Also these researchers argued that the growth impairment could be due to the energy costs of the photoacclimation process. In a similar manner, it can be argued that high light cells expend more energy on mechanisms to survive under high irradiance and other related metabolic processes that are less active under low light conditions. These processes also might not cease immediately after a transfer to a mass culture, therefore, it may take some time before normal mass culture efficiency is achieved. If the metabolic costs of the photoacclimation process are indeed high, it would partly explain the lower oxygen production rate in the BOM experiment. The higher requirement for ATP can be satisfied by burning sugars which leads to a higher oxygen consumption rate for high light acclimated cells as was, indeed, observed. Still, this would only be a partial explanation as the gross oxygen production (OCR + OPR) was also not substantially higher compared to the cells acclimated to mass culture conditions.

Apparently, the nature of the photoacclimation process hampers its advantageous utilization under mass culture conditions. Completely different cellular properties are required to efficiently utilize the light energy under low and high irradiance. In a mass culture, microalgae cannot specialize to a certain irradiance and will, therefore, always 
be easily oversaturated when having a high pigment content or possibly adopt a light wasting phenotype when being high light acclimated. Clearly, microalgae adjust their pigment content to the observed light intensity, but it remains unclear how the dynamics of a mass culture are perceived and translated into a certain pigment content (Grima et al. 1996) since the exact molecular mechanism behind irradiance dependent antenna size regulation has not yet been elucidated (Escoubas et al. 1995). However, it could still be possible to profit from local acclimation to the perceived light conditions by employing alternative microalgae cultivating methods. By growing microalgae immobilized in biofilms (Berner et al. 2014, Blanken et al. 2014, Wang et al. 2015), or compartmentalized in a photobioreactor (Grobbelaar et al. 2003), and allowing local acclimation to a fixed light intensity or a light intensity with minimal fluctuations, cells will acclimate to the light intensity at which they are cultivated (Al-Najjar et al. 2012, Li et al. 2016). This permits specialization, with cells under low light having a high chlorophyll content but less auxiliary light energy dissipating pigments and no activated dissipation mechanisms. Likewise, only the very high light acclimated cells will have a low pigment content and dissipation mechanisms that are essential for their position in the algae biofilm or photobioreactor.

\subsection{Conclusions}

In this study, we have measured the areal biomass productivity of high light acclimated cells of $C$. sorokiniana under mass culture conditions. The low-pigmented cells were compared to mass culture acclimated cells using two different methods. The short HRTreactor allowed a long-term productivity measurement of the low-pigmented cells with a retention time of two hours which was enough time to partly maintain its high light phenotype. In the biological oxygen monitor, the short-term oxygen production rate of low-pigmented cells was measured under simulated mass culture conditions. Based on model estimations, the reduced absorption cross section was expected to decrease oversaturation and wasting of light energy, resulting in a higher productivity. However, improved productivity of low-pigmented cells under mass culture conditions was not observed in any of the experiments. No solid explanation can be given based on the available experimental data. Two possible scenario were discussed. The most likely explanation is that the high light phenotype was accompanied by an increased capacity 
and tendency to thermally dissipate light energy. Another possibility is that a considerable amount of energy was expended on the photoacclimation process itself thereby counterbalancing the theoretical gain in light use efficiency. The results demonstrate that high light acclimated cells do not increase productivity when placed under mass culture conditions. Highest biomass yield on light energy was observed for the high light acclimated cultures. Therefore, local light acclimation could be beneficial when applying alternative cultivation methods such as immobilized growth in biofilms. 


\section{Appendix A: Microalgae growth model for reactor productivity estimation}

A kinetic model has been adopted to facilitate predicting the biomass productivity in a photobioreactor as a function of the local light intensity and light spectrum. The model comprises two compartments: the chloroplast and the cell content outside the chloroplast. In the chloroplast, 3-carbon sugars (triose) are produced. In this context, we express photosynthetic sugar production in units of the 1-carbon sugar equivalent $\mathrm{CH}_{2} \mathrm{O}$ that was formed. Sugar production in the chloroplast $\left(q^{c} \mathrm{CH}_{20}, \mathrm{~mol}_{\mathrm{CH} 2 \mathrm{O}} \mathrm{mol}_{\mathrm{x}}{ }^{-1} \mathrm{~S}^{-1}\right)$ is driven by light energy and can be described by the hyperbolic tangent model of Jassby and Platt (Jassby et al. 1976):

$q_{C H 2 O}^{c}=q_{C H 2 O, m}^{c} \cdot \int_{0}^{d} \tanh \left(\frac{\sum_{\lambda=400}^{\lambda=700} Y_{C H 2 O / p h, m} \cdot q_{p h, \lambda}(z) \cdot \Delta \lambda}{q_{C H 2 O, m}^{c}}\right) \cdot d z$

In this equation, $q \varepsilon_{\mathrm{H} 2 \mathrm{O}, \mathrm{m}}$ is the maximal sugar production rate $\left(\mathrm{mol}_{\mathrm{CH} 2 \mathrm{O}} \mathrm{mol}_{\mathrm{x}}{ }^{-1} \mathrm{~s}^{-1}\right)$ in the chloroplast; $Y_{\mathrm{CH} 2 \mathrm{O} / \mathrm{ph}, \mathrm{m}}$ is the maximum yield of sugar on light energy $\left(\mathrm{mol}_{\mathrm{CH} 2 \mathrm{O}} \mathrm{mol}_{\mathrm{ph}}{ }^{-1}\right)$, and $q_{p h, \lambda}(\mathrm{z})$ is the biomass specific light absorption rate $\left(\mathrm{mol}_{\mathrm{ph}} \mathrm{mol}_{\mathrm{x}}{ }^{-1} \mathrm{~s}^{-1}\right)$ at reactor depth $z$, which is defined, as follows, in Equation A2:

$q_{p h, \lambda}(z)=a_{x, \lambda} \cdot I_{p h, \lambda}(z)=\frac{I_{p h, \lambda}(z-d z)-I_{p h, \lambda}(z)}{C_{x} \cdot d z}$

Where $I_{p h, \lambda}(\mathrm{z})$ is local light intensity at reactor depth $z ; C_{X}$ is the biomass concentration in the reactor. $I_{p h, \lambda}(\mathrm{z})$ is calculated by the following equation according to the LambertBeer Law:

$I_{p h, \lambda}(z)=I_{p h, \lambda}(0) \cdot e^{-a_{x, \lambda} \cdot C_{x} \cdot z \cdot f_{l}}$

In this equation, $I_{p h, \lambda}(0)$ is the incident light intensity at the illuminated surface of the reactor, $a_{x, \lambda}\left(\mathrm{m}^{2} \mathrm{~mol}_{\mathrm{x}}{ }^{-1}\right)$ is the biomass absorption cross section, and $f_{l}$ the light path correction factor. This factor accounts for the influence of light scattering within the microalgae suspension and the fact that the incident light is not perfectly collimated. The overall effect is that the path travelled by the light rays within the algae suspension is longer than what would have been the case for light entering perpendicular to the reactor surface and with the absence of light scattering within the microalgae suspension. A longer light path increases the probability of light absorption, therefore, 
the $f_{l}$ factor increases the accuracy of the Lambert-Beer equation shown above. The $f_{l}$ parameter (Table A1) was fitted to the actual light intensity measurements performed in reactors containing a microalgae mass culture. Please refer to appendix D for a detailed description of the $f_{l}$ parameter estimation.

The cell content minus the chloroplast was defined as the second compartment of this model. In this aspect, part of the 3-carbon sugar is used for the production of new biomass at specific growth rate $\mu$. Another part of the sugar is respired in the mitochondria to provide energy in the form of ATP to support the growth reactions and to fulfil the maintenance requirements. The following equation was derived from a substrate balance over the cell minus chloroplast compartment where the maintenance requirement is fulfilled by substrate consumption according to Pirt (1965).

$\mu=\left(q_{C H 2 O}^{c}-m_{C H 2 O}\right) \cdot Y_{x / C H 2 O}$

and:

$\mu_{m}=\left(q_{C H 2 O, m}^{c}-m_{C H 2 O}\right) \cdot Y_{x / C H 2 O}$

Here, $m_{C H 2 O}$ is the biomass specific maintenance rate $\left(\operatorname{mol}_{\mathrm{CH} 2 \mathrm{O}} \mathrm{mol}_{\mathrm{x}}{ }^{-1} \mathrm{~s}^{-1}\right) ; Y_{\mathrm{X}} / \mathrm{CH} 2 \mathrm{O}$ is the biomass yield on sugar $\left(\operatorname{mol}_{\mathrm{x}} \operatorname{mol}_{\mathrm{CH} 2 \mathrm{O}^{-1}}\right)$; and $\mu_{m}$ is the maximum biomass specific growth rate $\left(\mathrm{s}^{-1}\right)$.

The biomass yield on light energy at each point in the reactor $\left(Y_{x / p h}(\mathrm{z}), \operatorname{mol}_{\mathrm{x}} \mathrm{mol}_{\mathrm{ph}}{ }^{-1}\right)$ can be calculated by employing the following equation:

$Y_{x / p h}(z)=\frac{\mu(z)}{q_{p h}(z)}$

Using equation A1 through A4, $\mu$ can be computed for every light intensity $I_{p h}$ at every location inside a flat panel photobioreactor culture illuminated from one side. Values for $\mathrm{a}_{\mathrm{x}, \lambda}, I_{p h, i n}, Y_{C H 2 O / p h, m} q^{c} C H 2 O, m$, and $m_{C H 2 O}$ should be provided (see Table A1). The integral in Eq. A1 is easily solved by numerical integration over a large number of sublayers $(N)$ with depth $\Delta z$. This procedure was implemented in an MS Excel based calculation routine, and the use of 100 sublayers was demonstrated to result in sufficient accuracy of the model prediction. The average biomass specific growth rate was calculated as follows: 
$\bar{\mu}=\sum_{z=0}^{z=d} \mu(z) \cdot \frac{\Delta z}{d}$

where $\Delta z=\frac{d}{N}$ and $d$ is the reactor depth. The average $q_{p h}$ and $Y_{x / p h}$ can be calculated using the same procedure.

The areal biomass productivity $\left(r_{x}, \mathrm{~g} \mathrm{~m}^{-2} \mathrm{~d}^{-1}\right)$ in a photobioreactor is the product of the biomass concentration $\left(C_{x}, \operatorname{mol}_{\mathrm{x}} \mathrm{L}^{-1}\right)$, reactor depth $d$, and the average biomass specific growth rate $\bar{\mu}$ :

$r_{x}=C_{x} \cdot d \cdot \bar{\mu}$

To maximize productivity, a biomass concentration must be found such that, at the back of the reactor, the maintenance rate $\left(m_{\mathrm{CH} 2 \mathrm{O}}\right)$ equals the local sugar production rate $\left(q \varepsilon_{\text {нго }}(d)\right)$. In this situation, the light intensity at the backside is equal to the compensation point and the net photosynthesis rate is equal to zero. This means that, in all locations in the photobioreactor, the biomass is positively contributing to biomass productivity, and there is no dark zone. Using an iteration method, $r_{x}$ can be maximized by incrementally changing the $C_{x}$ value for every repetition until it has converged to the optimal value. In this study, the MS Excel 'Solver' (GRG nonlinear algorithm) was employed.

Table A1 Overview of the model parameters.

\begin{tabular}{|c|c|c|c|c|}
\hline Parameter & Value & Unit & Description & References \\
\hline$\mu_{m}$ & 0.27 & $\mathrm{~h}^{-1}$ & Maximal biomass specific growth rate & $\begin{array}{c}\text { Uanssen et al. } 1999, \\
\text { Van Wagenen et al. } \\
\text { 2014) }\end{array}$ \\
\hline$q \varepsilon_{\mathrm{H} 20, m}$ & $1.11 \cdot 10^{-4}$ & $\begin{array}{l}\operatorname{mol}_{\mathrm{CH} 20} \\
\mathrm{~mol}_{\mathrm{x}}^{-1} \mathrm{~s}^{-1}\end{array}$ & $\begin{array}{l}\text { Maximal 3-carbon sugar production rate; } \\
\text { calculated from } \mu_{m} \text { using eq. A5. }\end{array}$ & \\
\hline$Y_{C H 2 O / p h, m, \lambda}$ & 0.11 & $\begin{array}{l}\operatorname{mol}_{\mathrm{CH} 20} \\
\operatorname{mol}_{\mathrm{ph}^{-1}}\end{array}$ & Maximum yield of sugar on light energy & $\begin{array}{l}\text { (Arnold 1949, Allen } \\
\text { 2003) }\end{array}$ \\
\hline$m_{\mathrm{CH} 2 \mathrm{O}}$ & $2.5 \cdot 10^{-6}$ & $\begin{array}{l}\operatorname{mol}_{\mathrm{CH} 20} \\
\mathrm{~mol}_{\mathrm{x}}^{-1} \mathrm{~S}^{-1}\end{array}$ & Biomass specific maintenance rate & (Blanken et al. 2016) \\
\hline$Y_{x / \mathrm{CH} 2 \mathrm{O}}$ & 0.70 & $\underset{\operatorname{mol}_{\mathrm{CH} 2 \mathrm{O}^{-1}}}{\operatorname{mol}_{\mathrm{x}}}$ & $\begin{array}{l}\text { Biomass yield on 3-carbon sugar using } \\
\text { ammonia as N-source }\end{array}$ & (Lee et al. 1996) \\
\hline$a_{x, \lambda}$ & - & $m^{2} g^{-1}$ & Absorption cross section & This paper \\
\hline$M_{x}$ & 24 & $\mathrm{~g} \mathrm{~mol}_{\mathrm{x}}{ }^{-1}$ & $\begin{array}{l}\text { Biomass dry weight to C-mol conversion } \\
\text { factor }\end{array}$ & (Duboc et al. 1999) \\
\hline$f_{l}$ & 1.74 & - & Light path correction factor & Appendix D, this paper \\
\hline
\end{tabular}




\section{Appendix B: Estimation of incident light intensity in the biological oxygen monitor}

The exact light intensity could not be measured because the used cuvette was too small to fit a light sensor. Therefore, the light intensity was estimated based on light measurements in the water jacket, in air (arrow 1 and 2 in Fig. A1). The measurement indicated by arrow 2 was performed after the cuvette was removed. Apparently the light intensity decreases with a factor 0.815 in the water jacket. We assume that, when filled with water, the same factor is applicable.

Correcting for reflection losses $(2000 * 1.0417 * 0.996), 2075 \mu \mathrm{mol}$ photons $\mathrm{m}^{-2} \mathrm{~s}^{-1}$ were estimated to enter the water jacket filled with water. We subsequently applied the 0.815 factor which is most likely caused by divergence of the light. An amount equal to $1691 \mu \mathrm{mol}$ photons $\mathrm{m}^{-2} \mathrm{~s}^{-1}$ will remain after passing the water in the jacket. Again, we corrected for reflection losses $(1691 * 0.996 * 0.96)$ caused by the glass plate between the jacket and the growth chamber to determine $1617 \mu \mathrm{mol}$ photons $\mathrm{m}^{-2} \mathrm{~s}^{-1}$ as the light intensity entering the growth chamber (See arrow no. 3). This is the light intensity that would have been measured (in air) if a light sensor would have fit into the cuvette. The other light measurements described in this paper are also based on measurements in air.

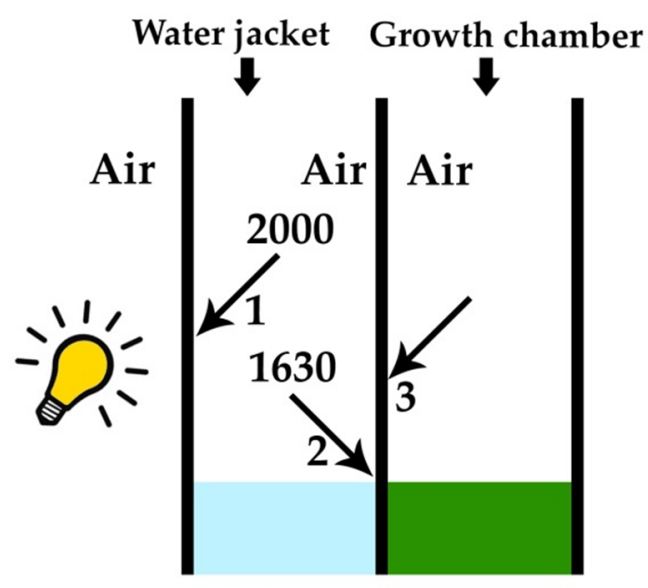

Fig. A1 Schematic overview of the light measurements that were performed to estimate the incident light intensity in the growth chamber of the biological oxygen monitor. The depicted values are light intensities in $\mu \mathrm{mol}$ photons $\mathrm{m}^{-2} \mathrm{~s}^{-1}$. The compartment on the right is the growth chamber, and the left compartment is the water cooling jacket. All measurements were performed in empty compartments in air. 


\section{Appendix C: conversion of oxygen production rate to biomass productivity}

According to the following stoichiometry (eq. A9) of the photosynthesis reaction, new biomass and oxygen are produced in a molar ration of approximately 1.11 to 1 . The elemental composition is based on Chlamydomonas (Kliphuis et al. 2012) and is assumed to be appropriate for Chlorella sorokiniana as well.

$0.93 \cdot \mathrm{CO}_{2}+0.654 \cdot \mathrm{H}_{2} \mathrm{O}+0.011 \cdot \mathrm{H}^{+}+0.07 \cdot \mathrm{CH}_{4} \mathrm{ON}_{2}+0.011 \cdot \mathrm{H}_{2} \mathrm{PO}_{4}{ }^{-} \longrightarrow \mathrm{CH}_{1.62} \mathrm{O}_{0.41} \mathrm{~N}_{0.14} P_{0.011}+1.109 \cdot \mathrm{O}_{2}$

Using Eq. A9 and assuming $24 \mathrm{~g} \mathrm{~mol}_{\mathrm{x}}^{-1}$ as a conversion factor for biomass dry weight to C-moles (Duboc et al. 1999), the oxygen production rates ( $\mu \mathrm{mol} \mathrm{O}_{2} \mathrm{~L}^{-1} \mathrm{~min}^{-1}$ ) could be converted to the volumetric biomass production rate (g biomass $\mathrm{L}^{-1} \mathrm{~min}^{-1}$ ) in the $\mathrm{BOM}$ cuvette. To obtain the areal productivity, the volumetric rate had to be multiplied with $1000 \mathrm{~L} \mathrm{~m}^{-3}$, and multiplied with the optical depth of the cuvette which was $0.02 \mathrm{~m}$ (please note that the optical depth is equal to the volume/area ratio). After multiplying by $1440 \mathrm{~min}^{-1}$, the areal biomass productivity $\left(r_{x}, \mathrm{~g} \mathrm{~m}^{-2} \mathrm{~d}^{-1}\right)$ was obtained. 


\section{Appendix D: Light path correction factor $\left(f_{i}\right)$ estimation}

In our model, a light path correction factor $\left(f_{l}\right)$ was used to account for the influence of light scattering within the microalgae suspension and the fact that the incident light is not perfectly collimated. The effect is that the path travelled by the light rays within the algae suspension is longer than that which would have been the case for light entering perpendicular to the reactor surface and with the absence of light scattering within the microalgae suspension. A longer light path increases the probability of light absorption, therefore, the $f_{l}$ factor increases the accuracy of the Lambert-Beer equation that was used in the model. The $f_{l}$ parameter was fitted to actual light intensity measurements as presented in Appendix E.

The fitting of the $f_{l}$ factor was performed as follows. Fig. A2. shows an example of typical light measurements that were performed with an ingoing light intensity of 1500 $\mu \mathrm{mol}$ photons $\mathrm{m}^{-2} \mathrm{~s}^{-1}$ and an outgoing light intensity of $10 \mu \mathrm{mol}$ photons $\mathrm{m}^{-2} \mathrm{~s}^{-1}$. First, it was determined what the ingoing and outgoing light intensity must have been at the front (see arrow no. 2) and back side (see arrow no. 3) of the algae suspension. The ingoing light intensity was measured behind the front glass plate in air (see arrow no. 1) which indicates that the actual light intensity in the algae suspension was higher (here, $1564 \mu \mathrm{mol}$ photons $\mathrm{m}^{-2} \mathrm{~s}^{-1}$ ) due to reflection losses. Reflection losses were compensated for by assuming $4 \%$ light loss for a glass/air surface and $0.4 \%$ loss for a glass/water surface.

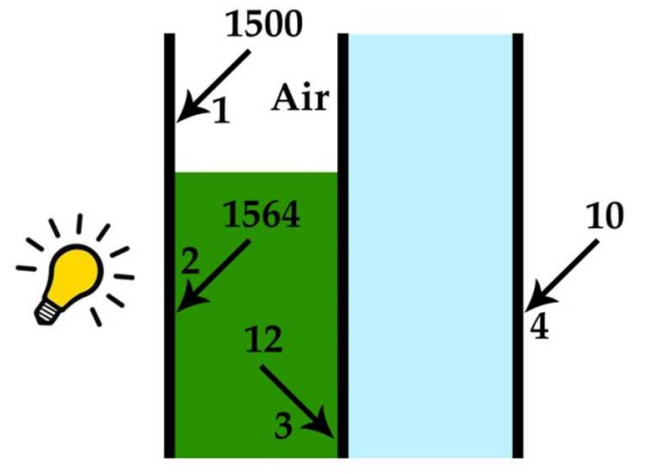

Fig. A2 Schematic overview of a typical reactor light measurement in air (Arrows 1 and 4) and the corresponding light intensities (after reflection and divergence correction, see text) at the front and back of the microalgae suspension (arrows 2 and 3). The depicted values are light intensities in $\mu \mathrm{mol}$ photons $\mathrm{m}^{-2} \mathrm{~s}^{-1}$. The left compartment is the microalgae suspension, and the compartment on the right is the water cooling jacket of an algaemist photobioreactor.

The light leaving the reactor behind the rear glass plate (see arrow nr. 4) was measured and could be converted to the light intensity at the back of the algae suspension (see arrow nr. 3). The value indicated by arrow 3 is thus estimated based on measurements 
performed at the location indicated by arrow 4 . In addition to reflection losses, the divergence of the light and the associated light absorption at the side walls of the reactor also account for losses. In a reactor that has both compartments filled with water, we measured $10 \%$ light loss within the cooling jacket which we assumed to be applicable as well to the situation where the growth chamber of the reactor contains an algae suspension.

The effect of divergence and associated absorption of light at the side walls of the growth chamber was neglected since we fitted the $f_{l}$ factor using two reactor experiments with a high biomass concentration (2.3 and $4.4 \mathrm{~g} \mathrm{~L}^{-1}$, respectively). We assumed that most light was absorbed by the algae before reaching the side walls or other obstacles (pipes, probes, cleaning magnet) in the reactor. We also assumed that a high biomass density results in the best estimation of the $f_{l}$ factor as light absorption by microalgae will be most dominant in this situation.

Using the Lambert-Beer Law and input data from two mass culture experiments with a high biomass density, the $f_{l}$ factor could be calculated.

$I_{p h, \lambda}(z)=I_{p h, \lambda}(0) \cdot e^{-a_{x, \lambda} \cdot C_{x} \cdot z \cdot f_{l}}$

We found $f_{l}$ values of 1.83 and 1.65 and decided to use the average (1.74) for our model estimations. 


\section{Appendix E: light measurements and experimental results}

Table D1 Overview of light measurements and experimental results of the short HRT experiment and the mass cultures that were used for comparison. Measurements are abbreviated as follows: $M C=$ Mass culture, MCLP = Mass culture with low-pigmented cells, $H L=$ High light culture with double sided illumination. $N$ represents the number of days of measurement. For the HL cultures, two values are given because two light sources were used for illumination.

\begin{tabular}{|c|c|c|c|c|c|c|c|c|c|c|}
\hline Experiment & 1 & 2 & 3 & 4 & 5 & 6 & 7 & 8 & 9 & 10 \\
\hline Description & $\mathrm{MC}$ & $\mathrm{MC}$ & $\mathrm{MC}$ & $\mathrm{MC}$ & MCLP & MCLP & $\mathrm{HL}$ & $\mathrm{HL}$ & $\mathrm{HL}$ & $\mathrm{HL}$ \\
\hline Iph,in $\left(\mu \mathrm{mol} \mathrm{m}{ }^{-2} \mathrm{~s}^{-1}\right)$ & 1502 & 1501 & 1511 & 1470 & 1518 & 1520 & $\begin{array}{l}1222 \\
1235\end{array}$ & $\begin{array}{l}1236 \\
1272\end{array}$ & $\begin{array}{l}1216 \\
1250\end{array}$ & $\begin{array}{l}1240 \\
1310\end{array}$ \\
\hline Iph,out $\left(\mu \mathrm{mol} \mathrm{m} \mathrm{m}^{-2}\right.$ & 11 & 12 & 10 & 10 & 12 & 12 & $\begin{array}{l}23 \\
27\end{array}$ & $\begin{array}{l}24 \\
28\end{array}$ & $\begin{array}{l}28 \\
24\end{array}$ & $\begin{array}{l}27 \\
23\end{array}$ \\
\hline $\mathrm{N}$ & 7 & 6 & 5 & 6 & 6 & 6 & 4 & 4 & 4 & 4 \\
\hline$C_{x}\left(\mathrm{~g} \mathrm{~L}^{-1}\right)$ & 2.27 & 2.38 & 2.5 & 2.2 & 3 & 3 & 2.9 & 3.1 & 2.7 & 2.7 \\
\hline SD & 0.12 & 0.07 & 0.07 & 0.11 & 0.06 & 0.07 & 0.03 & 0.06 & 0.07 & 0.03 \\
\hline$D\left(\mathrm{~d}^{-1}\right)$ & 3.7 & 3.3 & 2.9 & 3.7 & 12.8 & 12 & 5.7 & 5.2 & 5.7 & 6 \\
\hline SD & 0.03 & 0.12 & 0.18 & 0.17 & 0.2 & 0.34 & 0.11 & 0.07 & 0.08 & 0.07 \\
\hline$r_{x}\left(\mathrm{~g} \mathrm{~m}^{-2} \mathrm{~d}^{-1}\right)$ & 112 & 106 & 100 & 119 & 107 & 81 & 218 & 218 & 208 & 216 \\
\hline SD & 5.5 & 4.6 & 7.3 & 6.6 & 13.5 & 8.9 & 3.9 & 4.6 & 6.2 & 3.1 \\
\hline$Y_{x / p h}\left(\mathrm{~g} \mathrm{~mol}_{\left.\mathrm{ph}, \mathrm{in}^{-1}\right)}\right)$ & 0.87 & 0.83 & 0.77 & 0.94 & 0.82 & 0.62 & 1.05 & 1.03 & 1 & 1 \\
\hline SD & 0.04 & 0.04 & 0.06 & 0.05 & 0.1 & 0.07 & 0.02 & 0.02 & 0.03 & 0.01 \\
\hline
\end{tabular}

\begin{tabular}{lcc}
\hline Experiment & $\mathbf{1}$ & $\mathbf{2}$ \\
\hline \hline Light color & $\mathrm{MC}$ & $\mathrm{HL}$ \\
& & 1014 \\
Iph,in $\left(\mu \mathrm{mol} \mathrm{m}^{-2} \mathrm{~s}^{-1}\right)$ & 1501 & 653 \\
& & \\
Iph,out $\left(\mu \mathrm{mol} \mathrm{m}^{-2} \mathrm{~s}^{-1}\right.$ & 12 & 66 \\
$\mathrm{~N}$ & 6 & 6 \\
$C_{x}\left(\mathrm{~g} \mathrm{~L}^{-1}\right)$ & 2.38 & 1.51 \\
$\mathrm{SD}$ & 0.07 & 0.04 \\
$D\left(\mathrm{~d}^{-1}\right)$ & 3.3 & 6.2 \\
$\mathrm{SD}$ & 0.12 & 0.05 \\
$r_{x}\left(\mathrm{~g} \mathrm{~m}^{-2} \mathrm{~d}^{-1}\right)$ & 106 & 127 \\
$\mathrm{SD}$ & 4.6 & 4.38 \\
$Y_{x / p h}\left(\mathrm{~g} \mathrm{~mol}_{\text {ph,in }}{ }^{-1}\right)$ & 0.83 & 0.95 \\
$\mathrm{SD}$ & 0.04 & 0.03 \\
\hline
\end{tabular}

Table D2 Overview of light measurements and experimental results of the reactor cultivations that the biological oxygen measurements are based on. Measurements are abbreviated as follows: $M C=$ Mass culture, $H L=$ High light culture with double sided illumination. $N$ represents the number of days of measurement. For the HL cultures, two values are given because two light sources were used for illumination. 


\section{Appendix F: Light intensity distribution of light sources}

Table D1 Warm white incident light intensity ( $\mu \mathrm{mol}$ photons $\mathrm{m}^{-2} \mathrm{~s}^{-1}$ ) distribution over the reactor surface. The light was measured at 28 fixed points evenly distributed over the light exposed surface of the front glass panel of the culture chamber. The light was measured behind the front glass panel of a dummy reactor that lacks the other glass panels and reactor components, to make light measurements convenient. For this measurement, the average light intensity was $1501 \mu \mathrm{mol}$ photons $\mathrm{m}^{-2} \mathrm{~s}^{-1}$. Using colors the intensity distribution is visualized (darker = higher intensity).

\begin{tabular}{l|ccc}
\hline 1235 & 1429 & 1352 & 1286 \\
\hline 1464 & 1665 & 1656 & 1409 \\
\hline 1683 & 1837 & 1737 & 1739 \\
\hline 1586 & 1882 & 1953 & 1571 \\
\hline 1601 & 1738 & 1670 & 1438 \\
\hline 1383 & 1568 & 1591 & 1177 \\
\hline 1001 & 1135 & 1144 & 1099 \\
\hline
\end{tabular}


Chapter 4 
a $\sin \theta=m \lambda \quad(m=1,2,3)$ Inpen end Difroction groting $I=\operatorname{Im}\left(\frac{\sin A}{A}\right)^{2} \quad A=\frac{1}{2}$ $d \sin \theta=m \lambda(m=0,1,2)$ $f_{b e t}=\left|f_{1}-f_{2}\right| \quad f=\frac{\mu y}{4 L}\left(c=b^{2}, 3\right)$ disybrenot dectingde Ps

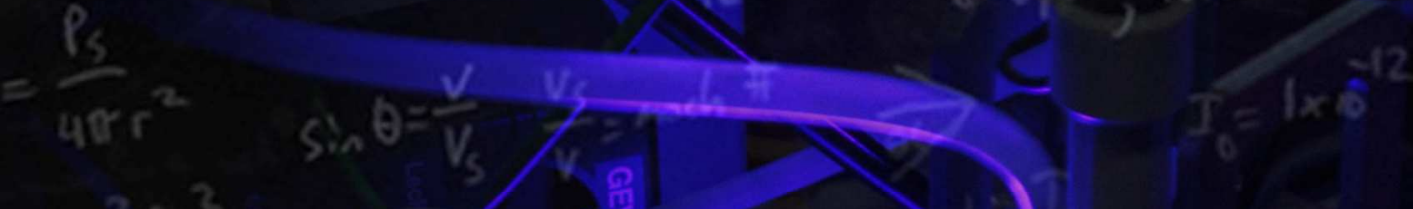
$2 v w^{2} s^{2}$

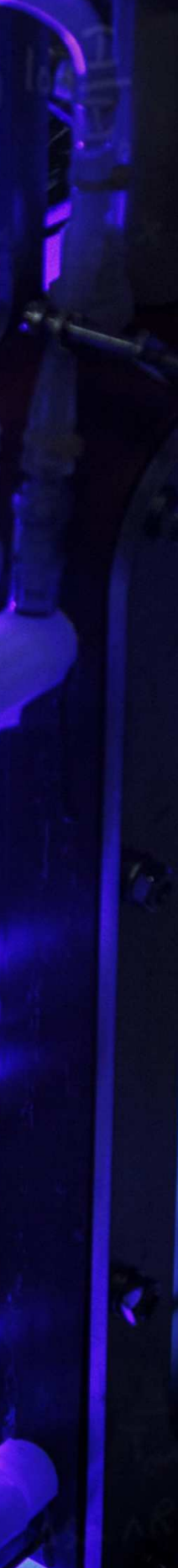

$D=\frac{m}{d \cos \theta}$ $f=\frac{\text { odius of corvoturt }}{2}$

t.)

hest $\Delta V$
$\Delta=1$
$y=1$
3
3
3 $f=\frac{v \pm v_{3}}{v \pm v_{5}}=0$

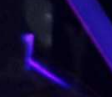
Ait syerction

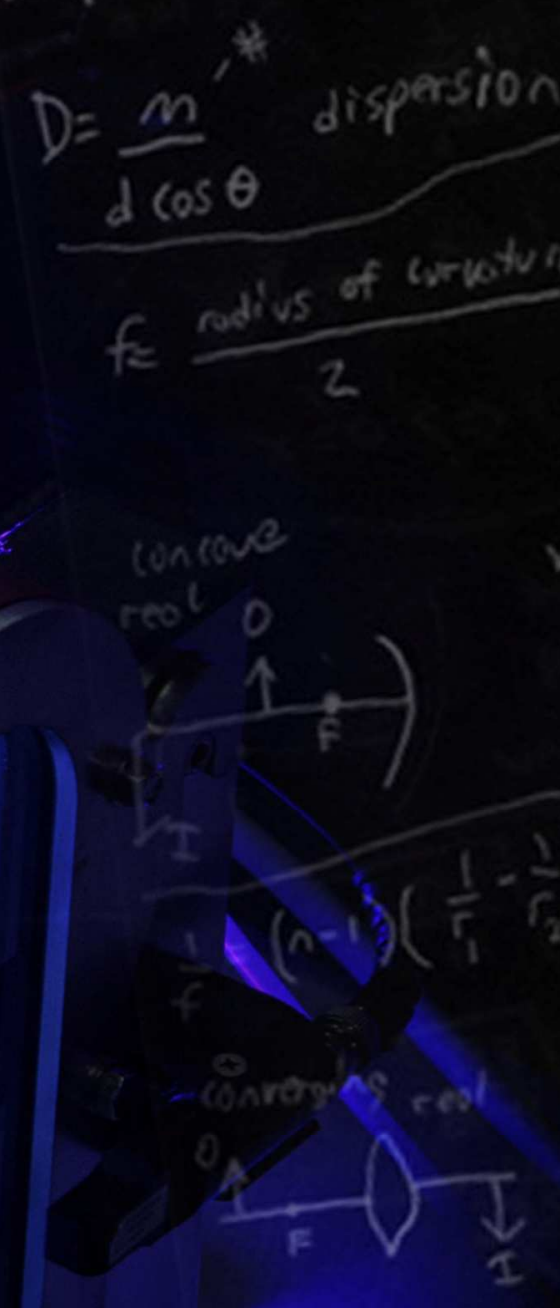

AT (con pross)

$\Delta V=A R D T($ co $p$ h)

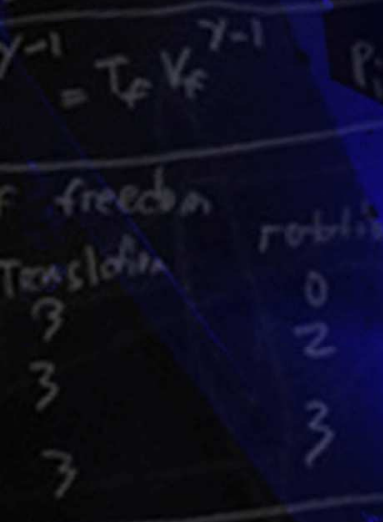

$$
n_{\theta}=\frac{25 \mathrm{~cm}}{f}
$$




\section{Chapter 5:}

Competition between antenna size mutant and wild type microalgae in mass culture

Tim de Mooij, Kira Schediwy, René H. Wijffels, Marcel Janssen 


\begin{abstract}
Under high light conditions, microalgae are oversaturated with light which significantly reduces the light use efficiency. Microalgae with a reduced pigment content, antenna size mutants, have been proposed as a potential solution to increase the light use efficiency. The goal of this study was to investigate the competition between antenna size mutants and wild type microalgae in mass cultures. Using a kinetic model and literature-derived experimental data from wild type Chlorella sorokiniana, the productivity and competition of wild type cells and antenna size mutants were simulated. Cultivation was simulated in an outdoor microalgal raceway pond production system which was assumed to be limited by light only. Light conditions were based on a Mediterranean location (Tunisia) and a more temperate location (the Netherlands). Several wild type contamination levels were simulated in each mutant culture separately to predict the effect on the productivity over the cultivation time of a hypothetical summer season of 100 days. The simulations demonstrate a good potential of antenna size reduction to increase the biomass productivity of microalgal cultures. However, it was also found that after a contamination with wild type cells the mutant cultures will be rapidly overgrown resulting in productivity loss.
\end{abstract}




\subsection{Introduction}

One of the most important bottlenecks in microalgae cultivation is the inefficient utilization of light energy under high irradiance. Microalgae are easily oversaturated with light which strongly reduces the light use efficiency. This limitation can be alleviated by genetically reducing the amount of light energy that is absorbed per cell. The creation of such antenna size mutants, i.e., microalgae with a reduced pigment content, has been proposed as a potential solution (Mussgnug et al. 2007, Ort et al. 2011, Formighieri et al. 2012, Perrine et al. 2012, Kwon et al. 2013, Oey et al. 2013) to light saturation. The lower pigmentation of the mutants allows increased biomass concentrations with the same light gradient in the photobioreactor (Melis et al. 1998, Mussgnug et al. 2007). The lower biomass specific light absorption rate leads to a higher productivity of mutant cultures than that of wild type cultures under high light conditions (Mussgnug et al. 2007). In practice, some mutants were indeed reported to demonstrate improved growth characteristics under specific light conditions (Mussgnug et al. 2007, Mitra et al. 2008, Cazzaniga et al. 2014).

Clearly, antenna size mutant generation, especially through directed mutagenesis, is an immature technology (de Mooij et al. 2014). Currently there are no suitable antenna size mutant strains available that can be analyzed for their performance in competition experiments. A better understanding of the photosynthetic machinery and an advanced genetic toolbox are required to create better mutants. Still, it is interesting to study the potential of antenna size reduction for mass cultivation of microalgae using model simulations. Extrapolation of laboratory data utilizing a predictive model that is constructed on a solid theoretical foundation is an attractive alternative to performing outdoor cultivation experiments. Modeling can function as a great tool to investigate the improvement of mass culture productivity using antenna size mutants. In addition, the impact of competition for light between mutant and wild type strains can be predicted. Competition between the antenna size mutant and its own wild type is expected to result in productivity losses. The outcome of such a modeling approach results in more realistic expectations and can be used to determine genetic engineering targets to optimize mutant cultivation. 
The aim of this study was to estimate antenna size mutant productivity in photobioreactor mass cultures and quantify the competition between the antenna size mutant and its wild type. Knowledge on growth properties of separate cultures (Hobson 1969, Huisman et al. 1999) is required to analyze mixed cultures and to estimate the competitiveness of the involved species under light limiting conditions. In our study the productivity was calculated with a microalgal growth model which is based on summing up local rates of photosynthesis calculated for every position in the photobioreactor. The model takes into account the light gradient within the microalgal culture and also the wavelength dependency of light absorption. The model is based on a previously validated growth model for Chlorella sorokiniana under continuous light (Blanken et al. 2016), and it is extended with a carbon partitioning mechanism to describe growth under day/night cycles. The light model was further adapted to represent an outdoor microalgal raceway pond production system taking into account solar elevation. The wild type properties were defined based on experimental data of $C$. sorokiniana. Antenna size mutants were considered to only differ from the wild type in their reduced absorption cross section to clearly identify the potential of reduced antenna sizes. Productivities of the mutant monocultures with different antenna size reductions (090\%) were investigated. Several wild type contamination levels were separately simulated in each mutant culture to predict the effect on the productivity over the cultivation time (100 days).

\subsection{Model description}

\subsubsection{Structure}

With the kinetic model the biomass productivity of a microalgae mass culture is calculated as a function of the incident light intensity during the diurnal cycle. The change of the spectral composition with increasing reactor depth due to the preferential light absorption of microalgae was taken into account. The model allows for the calculation of biomass productivity of an antenna size mutant culture before, during, and after contamination with its wild type. In all of the cases, light is assumed to be the limiting factor for growth and, consequently, mutant and wild type are competing for light energy. An extensive description of the model can be found in Appendix A. 
To account for the effects of the diurnal cycle and carbon partitioning, functional biomass and intracellular carbohydrate reserves were regarded as separate compounds. The carbohydrate reserves are referred to as 'sugar' in this study. Consequently, for each strain, two production rates were distinguished: the production of functional biomass $\left(X^{\prime}\right)$ and of sugar $(S)$.

Refer to Fig. 5.1 for an overview of the model structure. The overall microalgae production model consists of three modules: Location, time, and wavelength dependent light distribution (Module 1); specific light absorption, and photosynthetic sugar production (Module 2); partitioning of sugar towards biomass, and a sugar reserve pool (Module 3). The first module describes the location-specific light intensity and the light distribution in the culture during the day. These are based on solar elevations, Snell's law, Fresnel's equations, and Lambert-Beer's law, while neglecting the effect of light scattering. The second module describes the sugar production as a function of specific light absorption and, consequently, depends on the local light intensity, biomass concentration, and strain-specific characteristics. The third module represents the allocation of photosynthetically produced sugar to maintenance related processes, to the accumulation of carbohydrate reserves, and to the production of functional biomass. In addition, the partitioning of sugar is differentiated between the day and night periods during the diurnal cycle.

\subsubsection{Strategy and assumptions}

The simulated cultivation time was 100 days. We simulated both a Mediterranean location (Tunisia, Lat. $36.867^{\circ}$ ) with a high irradiance and clear sky and a temperate location (the Netherlands, Lat. $52.117^{\circ}$ ) with considerable cloud cover. One specific summer day (July 15th, day 196 of the year) was continuously repeated in the model for each location. All simulations began at 8:00 am local solar time. During the day, the dilution rate was maintained constant while there was no culture dilution during the night. The dilution rate was optimized for each strain and each location (Netherlands and Tunisia) to maximize the productivity (See Appendix D for optimization procedure). The optimal dilution rate was subsequently applied during the final simulations. 
Theoretical antenna size mutants of Chlorella sorokiniana were simulated. The strainspecific characteristics $\left(\mu_{m}, m_{S}, Y_{X^{\prime} / S}, q_{S}^{\max }, x_{S, \min }\right)$ of the mutants were kept identical to the experimentally determined characteristics of the wild type (Table E3 of Appendix E), i.e., only the biomass specific absorption cross section $\left(a_{X^{\prime}}\right)$ was altered $(10-90 \%$ reduction), and $a_{X^{\prime}}$ was assumed to be constant throughout the entire day/night cycle. The wild type absorption cross section was measured under mass culture conditions

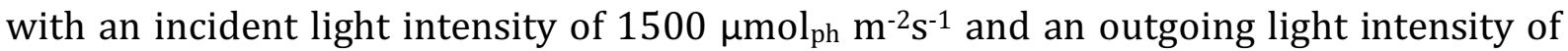
$10 \mu \mathrm{mol}_{\mathrm{ph}} \mathrm{m}^{-2} \mathrm{~s}^{-1}$. The antenna size mutation is assumed to be stable and not affecting any other cellular property, and the considered strains are assumed as not being sensitive to photoinhibition under the simulated light conditions. Different wild type contamination levels were considered as well as the scenario of a reverse mutation which was assumed to result in a contamination of one cell per cubic meter at the initiation of the 100-days simulation. One cell of $C$. sorokiniana corresponds to a mass of $1.4 \cdot 10^{-11} \mathrm{~g}$, or $0.14 \mathrm{pg}$ (Rosenberg et al. 2014). A reflective (80\%) ground cover was simulated to be situated at the bottom of the photobioreactor pond to allow a fairer comparison of mutant and wild type performance.

The areal biomass productivity $\left(r_{C x}\right)$ was calculated by multiplying the total biomass concentration $\left(X^{\prime}+S\right)$ with the dilution rate $(D)$ and the reactor depth $\left(d_{R}\right)$ :

$$
r_{C x}=\left(X^{\prime}+S\right) \cdot D \cdot d_{R}
$$




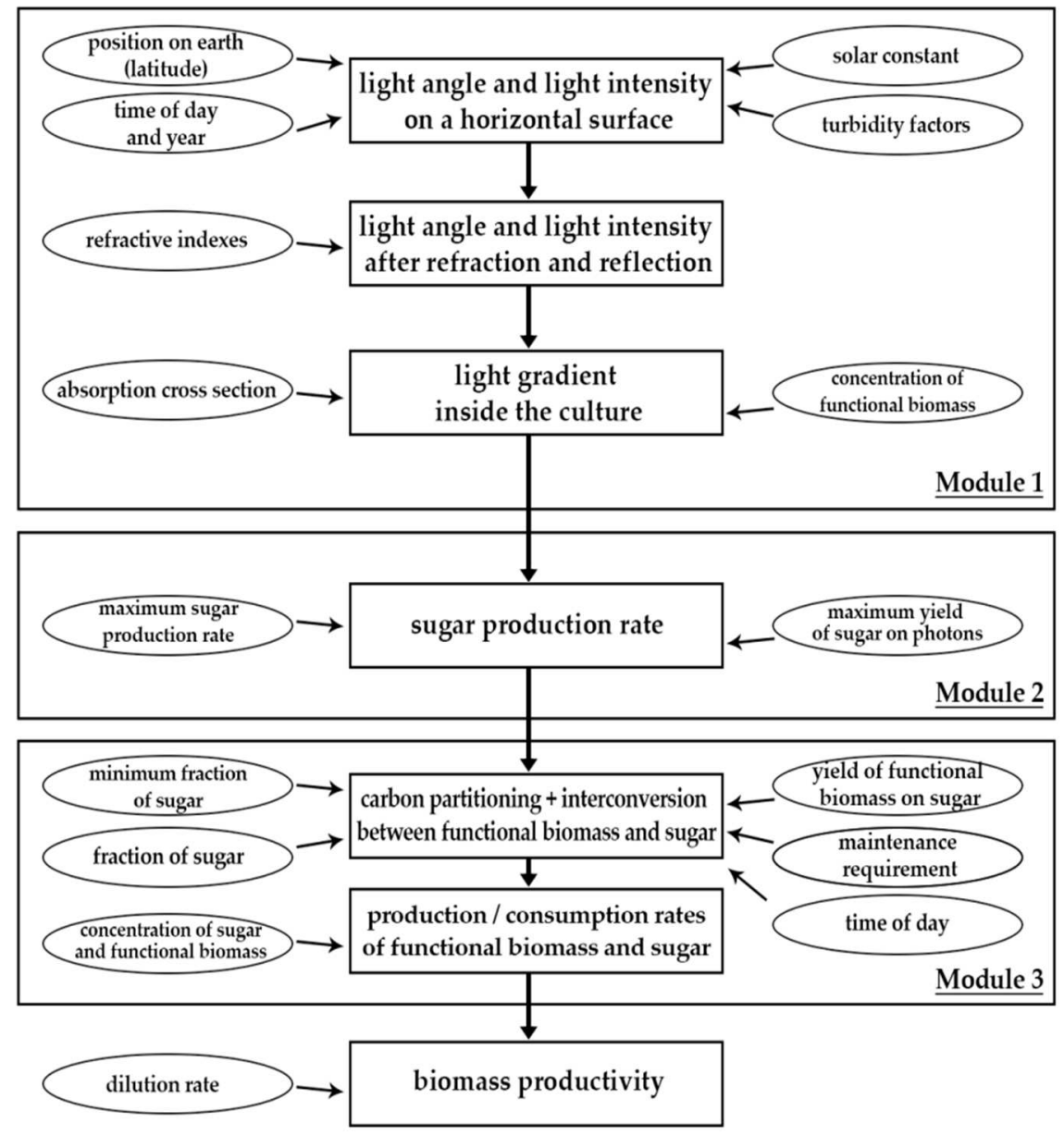

Fig. 5.1 Calculation scheme for the three modules. Module 1: location, time, and wavelength of specific light distribution; Module 2: photosynthetic sugar production; Module 3: sugar allocation towards biomass, the sugar reserve pool, and maintenance during the diurnal cycle. Model inputs are in ovals and calculated values in solid boxes.

\subsection{Results}

Simulations with the model were performed for monocultures of the wild type of Chlorella sorokiniana and for monocultures of the antenna size mutants with a varying absorption cross section $\left(a_{X^{\prime}}\right)$ in the range of a $10-90 \%$ reduction compared to the wild type. In addition, monocultures of the antenna size mutants were 'contaminated' with wild type cells at various contamination levels in order to investigate the competiveness of the mutants and the dynamics of outcompetition. The wild type and the mutants were compared regarding their areal biomass productivity at two locations, the Netherlands and Tunisia, during a hypothetical summer season of 100 days. Refer to Appendix $\mathrm{C}$ for the light pattern of one summer day for both locations that was employed in all simulated days to obtain repetitive light conditions. For contaminated 
cultures, the loss of productivity as a result of the contamination with the wild type was also investigated.

\subsubsection{Simulation overview}

In Fig. 5.2A, the day/night cycle of one cultivation is illustrated for both the wild type and a mutant with an $80 \%$ antenna size reduction in Tunisia. The net sugar production rate $\left(X^{\prime} \cdot\left(q_{S}-m_{S}\right)\right)$ is a measure for the photosynthetic activity of the mutant culture. It is illustrated that the net sugar production rate of the mutant culture is considerably higher than that of the wild type culture only during the brightest hours of the day. In the night the sugar production rate is negative because sugar is used to build new functional biomass and sugar is partly respired to support both the growth processes as well as the maintenance related processes. The mutant culture exhibits greater losses of photosynthetically derived sugar during the night because of the higher biomass concentration and the associated maintenance requirements that must be satisfied. 

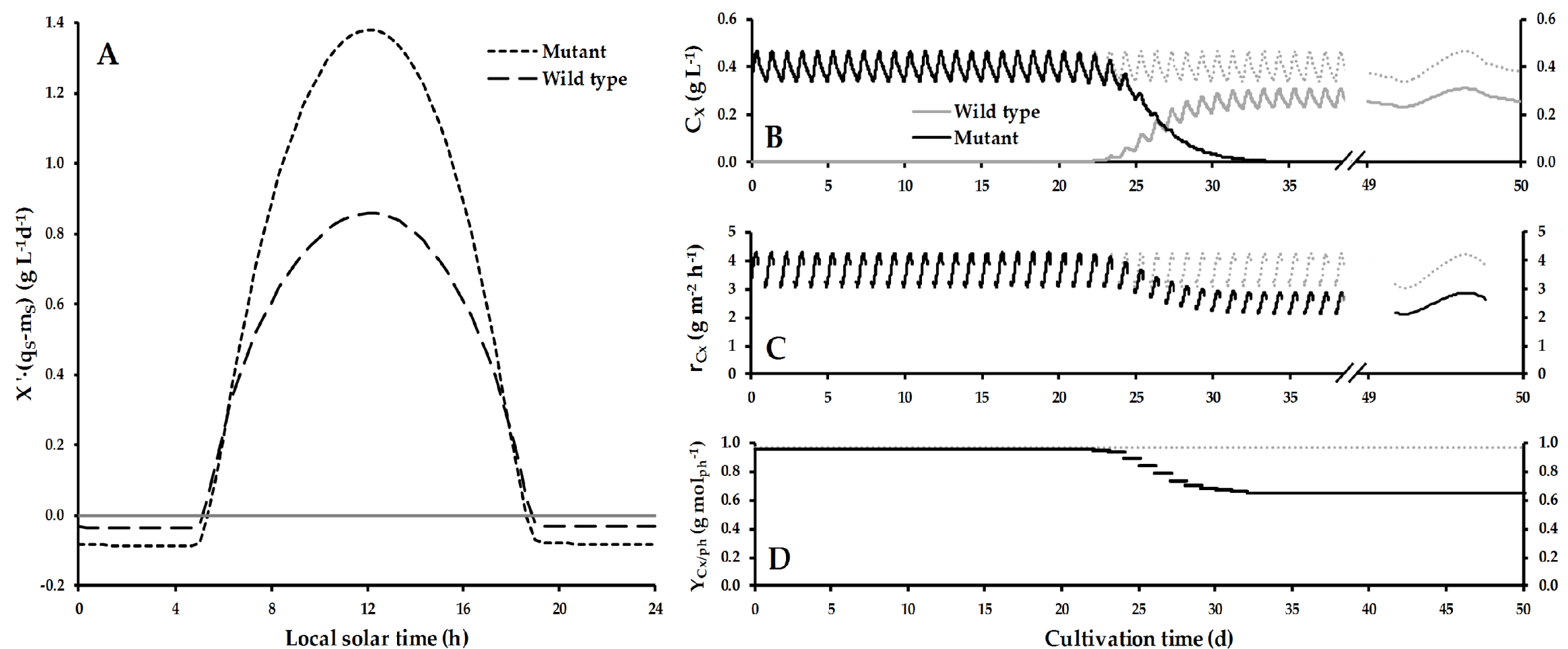

Fig. 5.2 (A) Net sugar production rate $\left(\mathrm{X}^{\prime} \cdot\left(\mathrm{q}_{\mathrm{s}}-\mathrm{m}_{\mathrm{s}}\right)\right)$, a measure for the photosynthetic activity minus the maintenance, presented for the duration of one day/night cycle. In B, C, and D, an overview is provided of the first $50 \mathrm{~d}$ of a $100 \mathrm{~d}$ simulation of an antenna size mutant with $80 \%$ reduction in the absorption cross section (ax') in Tunisia. Day 50 is plotted on a different scale in B and C to illustrate the variation over the course of one day in greater detail. The culture is contaminated at $t=0$ with one wild type cell per cubic meter. The dashed grey lines depict the scenario of an axenic mutant culture without contamination. (B) The biomass concentration ( $\left.\mathrm{C}_{\mathrm{x}}\right)$ of the mutant and the wild type. (C) The daily areal biomass productivity $\left(\mathrm{r}_{\mathrm{Cx}}\right)$ of the mixed culture as the sum of the productivity of mutant and wild type is depicted by the solid black line. (D) The biomass yield on incident light $\left(\mathrm{Y}_{\mathrm{Cx} / \mathrm{ph}}\right)$ averaged over one day is shown by the solid black line and is based on the mixed culture of wild type and mutant cells. The dilution rate was kept constant during the light period, and no dilution was applied during the night. 
Exemplary, the first 50 days of the 100 days cultivation period are shown for the mutant with an $80 \%$ antenna size reduction in Tunisia (Fig. 5.2B-D). As demonstrated later, a monoculture of this mutant results in the highest areal biomass productivity $\left(r_{C x}\right)$. It can be observed in greater detail on day 50 in Fig. 5.2B and 5.2C that, during the day, biomass is produced and the biomass concentration is increasing. However, in the morning and from 16:00 solar time onwards, the biomass concentration is decreasing as the result of the low incident light intensity in combination with culture dilution. The course of the cultivation is illustrated for monocultures of the mutant with and without contamination by the wild type. The shown contamination level is one wild type cell per cubic meter at day 0 . The contamination has substantial effects on the course of the cultivation after approximately 27 days (Fig. 5.2B). The mutant concentration then decreases rapidly while the wild type concentration increases, which considerably reduces the biomass productivity. The wild type reaches a lower biomass concentration and lower productivity than the mutant. These effects are a result of the wild type absorbing more light per cell which leads to more oversaturation and light loss. This is also manifested as a decrease of the biomass yield on light (Fig. 5.2D).

\subsubsection{Monocultures of antenna size mutants and their wild type}

The areal biomass productivity of antenna size mutants in a simulated outdoor raceway pond photobioreactor was investigated. The reduction in absorption cross section was normalized to the wild type, and a reduction of 10 to $90 \%$ was simulated. The areal biomass productivity of the mutant monocultures was found to increase with increasing antenna size reduction until a maximum is reached (Fig. 5.3A). The maximum productivities were found for cultures with antenna size reductions of $80 \%\left(53.2 \mathrm{~g} \mathrm{~m}^{-2} \mathrm{~d}^{-}\right.$ 1) in Tunisia and $60 \%\left(31.5 \mathrm{~g} \mathrm{~m}^{-2} \mathrm{~d}^{-1}\right)$ in the Netherlands. After the maximum, the biomass productivity decreases when the antenna size is further reduced.

Comparing the two locations, the areal biomass productivity was higher in Tunisia than in the Netherlands for all of the mutants and the wild type. Because of the higher irradiance, both the biomass concentrations and the optimal dilution rates were higher in Tunisia than in the Netherlands. In Tunisia, the increase of the productivity with the 
antenna size reduction is steeper and leading to a sharper maximum than in the Netherlands. A relative productivity increase of $39 \%$ in Tunisia and of $16 \%$ in the Netherlands was observed for the best performing mutant strain.

The loss in productivity after the maximum has been reached is primarily caused by the fact that light, after being reflected at the bottom due to the ground cover (i.e. liner), is leaving the photobioreactor pond unused at the surface when applying a very high antenna size reduction. It is important to note that a white liner was simulated with an $80 \%$ reflectivity as described in Appendix B. For an antenna reduction of $90 \%$ in Tunisia, up to $100 \mu \mathrm{mol}_{\mathrm{ph}} \mathrm{m}^{-2} \mathrm{~s}^{-1}$ is lost in this way at solar noon. In addition, the higher costs for maintenance required for the high biomass concentration further decrease the productivity. The maintenance requirement is assumed to be a constant and equal for all mutants and the wild type. Consequently, the relative maintenance costs increase up to $24 \%$ in the Netherlands for a $90 \% a_{X^{\prime}}$ reduction (See Fig 5.3A). At moderate antenna size reductions only $10 \%$ of the total produced sugar is consumed for the purpose of maintenance. This means that at high antenna size reduction a rapidly increasing fraction of photosynthetically produced sugar is used for maintenance instead of being incorporated into new biomass. The effect of maintenance is higher for lower irradiance conditions as present in the Netherlands.

The relation between antenna size reduction and photosynthetic efficiency under saturating light conditions is also reflected in the biomass yield on light (Fig. 5.3B). Because of the higher irradiance in Tunisia, overall yields are lower than in the Netherlands. Only beyond $80 \%$ antenna size reduction the biomass yield on light was higher in Tunisia than in the Netherlands. For small antennae, light oversaturation in Tunisia is relatively low while the light levels during the majority of the day are above

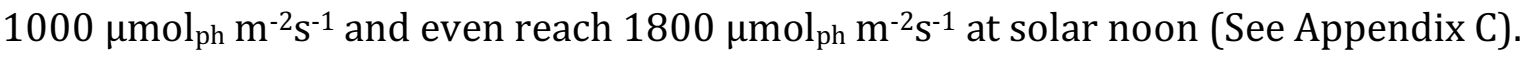



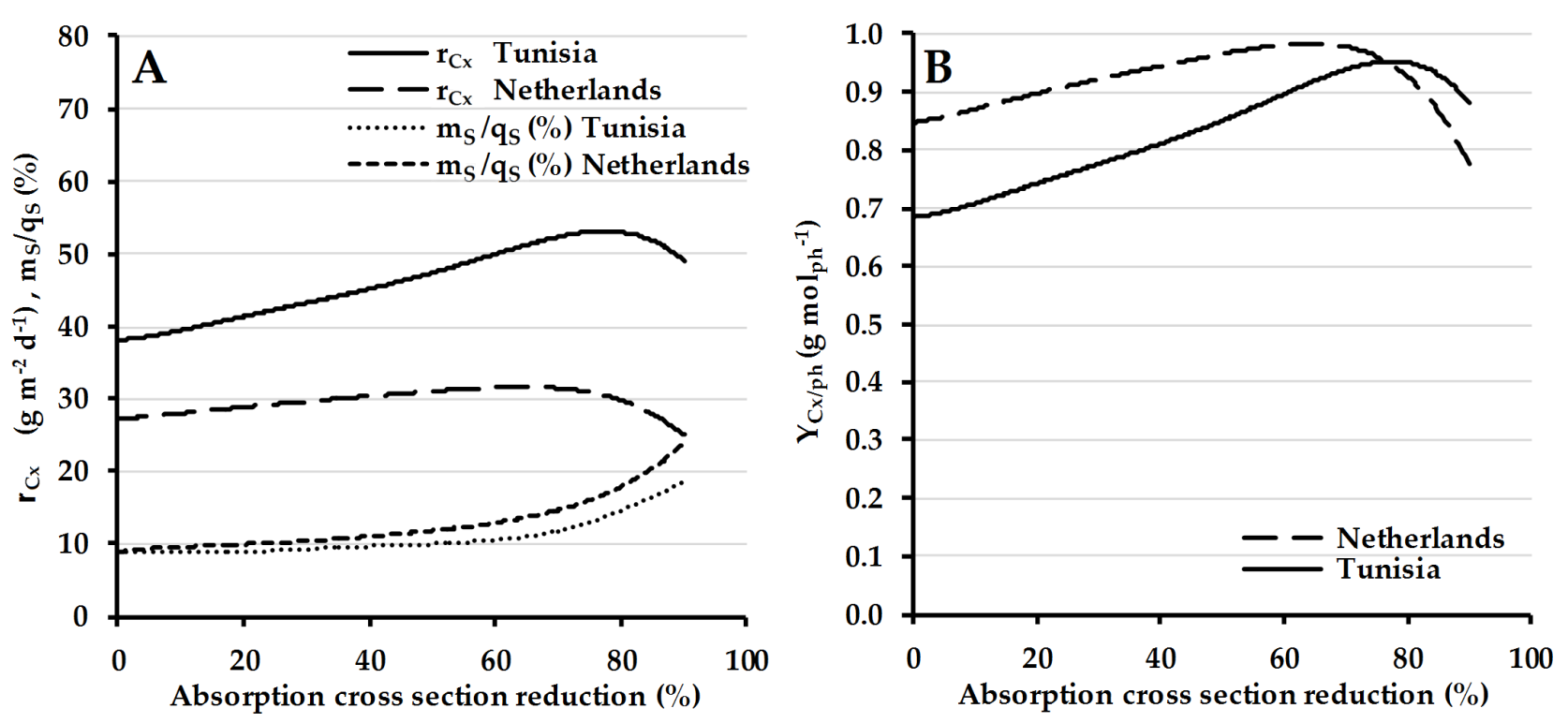

Fig. 5.3 (A) Biomass productivity $\left(r_{C_{x}}\right)$ and relative costs of maintenance processes $\left(m_{S} / q_{S}, \%\right)$, i.e the fraction of produced sugar used for maintenance and not for growth. (B) biomass yield on incident light $\left(Y_{C x / p h}\right)$ presented for the wild type (0\% reduction) and antenna size mutant cultures. For the calculation of the biomass yield on light, the daily averaged productivity is normalized to the daily amount of incident light on the photobioreactor.

\subsubsection{Culture dynamics after mutant monoculture contamination by wild type cells}

Antenna size mutant monocultures with a varying reduction in absorption cross section $\left(a_{X^{\prime}}\right)$ were simulated as being contaminated with different levels of wild type cells. In this way the rate at which mutant cultures are overgrown was investigated as a function of the contamination level and antenna size reduction. Fig. 5.4A illustrates the effect of different contamination levels on the time it takes for wild type cells to become the dominant strain in the culture. Only the results for Tunisia are shown. The optimal antenna size reduction for Tunisia, $80 \%$, was selected in order to study the effect of the contamination level. At the highest contamination level of $1 \%$, the decrease in mutant biomass concentrations becomes apparent already after three days. Lower contamination levels extend the duration that the antenna size mutant is the dominant strain and during this period light is used at a higher efficiency compared to the situation in which the wild type is the dominant strain. However, even in the best case scenario that was considered, with a contamination of only one cell per cubic meter, the mutants were overgrown before half of the simulated summer season (100 days) had passed. 

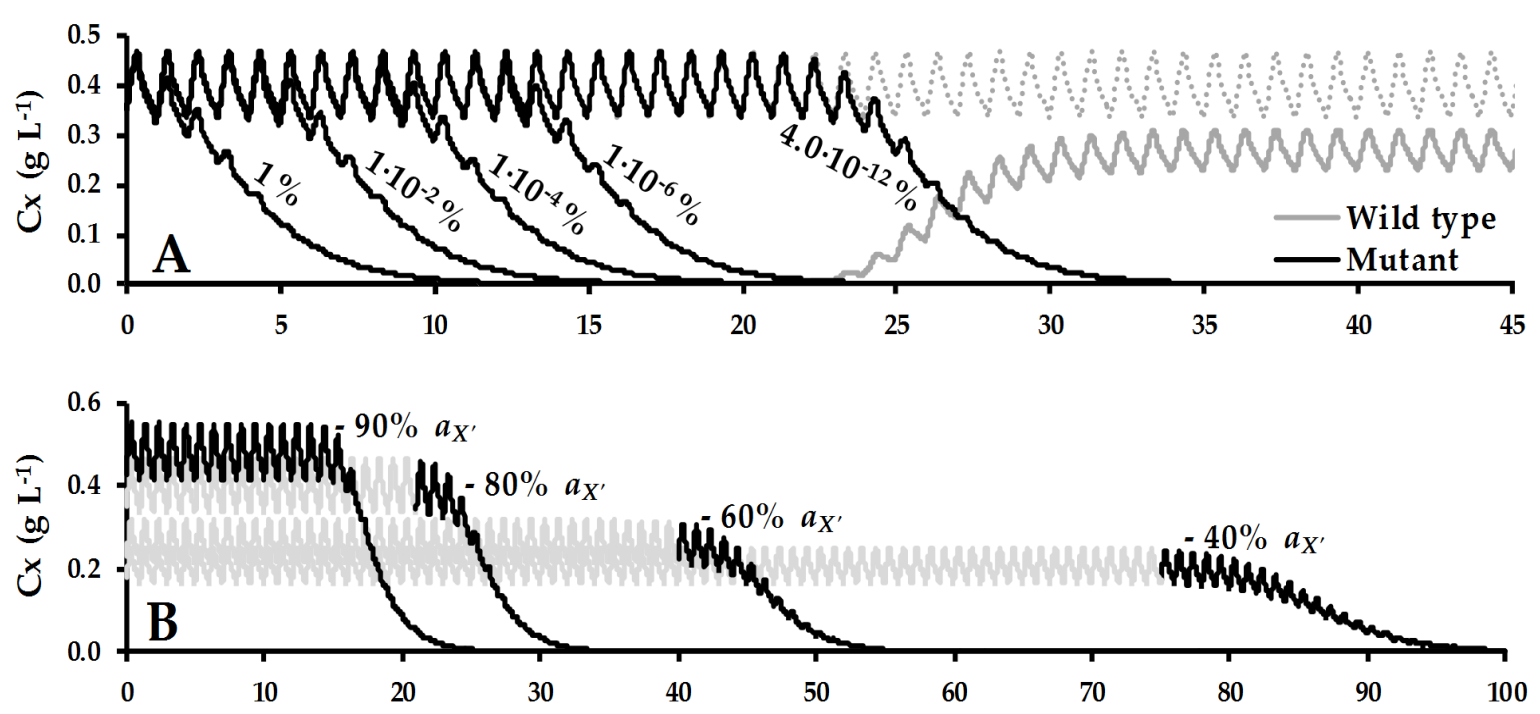

Fig. 5.4 (A) Effect of the contamination level on the rate at which antenna size mutants are overgrown by wild type cells in a simulated raceway pond photobioreactor with a depth of $0.2 \mathrm{~m}$, situated in Tunisia. The mutant monocultures have an $80 \%$ reduction in the absorption cross section $\left(\boldsymbol{a}_{X^{\prime}}\right)$. The dashed line indicates the scenario of a mutant monoculture with no contamination. Only for the lowest contamination level $\left(4.0 \cdot 10^{-12} \%\right.$, or 1 cell $\mathrm{m}^{-3}$ at day 0$)$ the associated increase in wild type biomass concentration is illustrated by the solid grey line. (B) Effect of antenna size reduction on the rate at which the mutant monocultures are overgrown by the wild type cells. For clarity reasons, the wild type concentration is not presented. The contamination level for these simulations equal one wild type cell per cubic meter at day 0.

In addition to the contamination level, the effect of the antenna size reduction was investigated (Fig. 5.4B). Again, one cell per cubic meter was chosen as the contamination level in a monoculture in Tunisia. Clearly, greater reductions in antenna size result in less competitive mutants and, therefore, the mutants with the highest potential in terms of mass culture productivity are most vulnerable and rapidly overgrown by wild type cells within one simulated summer season. For antenna size reductions below $40 \%$, the mutants remain the dominant strain in the culture during the simulated season. However, as shown before in Fig. 5.3, a 40\% reduced antenna size mutant does not exploit the full potential of antenna size reduction.

\subsection{Discussion}

In accordance with our expectations, the areal biomass productivity was higher in Tunisia than in the Netherlands for all mutants and the wild type. Up to a $39 \%$ increase in productivity was estimated for a $80 \%$ antenna size reduced mutant compared to the wild type. In contrast to Tunisia, in the Netherlands under the most optimistic conditions, only a $16 \%$ increase in productivity could be obtained compared to the wild 
type cultivation. These numbers emphasize that both a considerable antenna size reduction and high irradiance are required to obtain substantial gains in productivity. Most antenna size mutants that have been created do not exhibit such high antenna size reductions (de Mooij et al. 2014). In Tunisia, saturating light conditions are present over a longer period of the day than in the Netherlands. Therefore, any reduction in absorption cross section reduces oversaturation and thus increases light use efficiency (Melis et al. 1998, Mussgnug et al. 2007, Mitra et al. 2008, Zhu et al. 2010, de Mooij et al. 2014). The results show a clear potential for the use of antenna size mutants in high irradiance locations such as Tunisia.

The theoretical maximum biomass yield on light $\left(Y_{C x / p h}\right)$ for Chlorella sorokiniana with nitrate as nitrogen source is approximately $1.57 \mathrm{~g} \mathrm{~mol}_{\mathrm{ph}^{-1}}$ (Kliphuis et al. 2010). Compared to this value, the simulated yields for wild type cultivation in the Netherlands (0.85 $\mathrm{g} \mathrm{mol}_{\mathrm{ph}^{-1}}$ ) and Tunisia $\left(0.68 \mathrm{~g} \mathrm{~mol}_{\mathrm{ph}^{-1}}\right.$ ) show room for improvement. However, in large scale production, the theoretical maximum cannot be reached under saturating light. Using the optimal $80 \%$ antenna size reduction in Tunisia, the biomass yield on light energy was simulated to increase to $0.95 \mathrm{~g} \mathrm{~mol}_{\mathrm{ph}}{ }^{-1}$, which is high for large scale cultivation in an environment of high irradiance. The estimated productivities and biomass yields on light are in the expected ranges based on empirical data of $C$. sorokiniana (Zijffers et al. 2010), which supports the reliability of the presented results.

Smaller antenna sizes increase the productivity in environments of high irradiance. However, there is an upper limit because the higher the antenna size reduction is, the higher the optimal biomass concentration that is required to absorb the incident light intensity. At higher antenna size reductions, it becomes practically impossible to harvest all of the light that is available around solar noon. Consequently, especially green light is leaving the photobioreactor unused after being reflected at the bottom. In addition, $20 \%$ of the light that reaches the pond floor is absorbed by the pond liner which means that a more 'transparent' culture will also result in more loss of light at the bottom. Theoretically, the problem could be alleviated by adjusting the biomass concentration over the course of the entire day, however, in practice, it is impossible to maintain an optimal biomass concentration throughout the entire day as biomass growth is too slow 
to respond to sunrise, sunset and the fluctuation light conditions during the day (Pruvost et al. 2015).

As the maintenance requirement per cell is assumed to be constant, the total energy spent on maintenance increases linearly with the biomass concentration. In the range of $10-80 \%$ antenna size reduction, the increase in maintenance costs is not a big issue since the concomitant increase of the productivity is higher, and this outweighs the maintenance losses. However, as depicted in Fig. 5.3A, the fraction of sugars utilized for maintenance can be as high as $24 \%$ for a 90\% antenna size reduction in the Netherlands. Therefore, an optimal antenna size reduction was identified based on the model. In addition to this energetically optimal antenna reduction, in practice, antenna size reduction may possibly be limited by structural constraints as there appears to be a minimum amount of chlorophyll molecules that is required for the assembly of the photosystem core complexes (Glick et al. 1988).

A reflective ground cover (i.e., liner) was simulated to be situated at the bottom of the photobioreactor pond to allow a fairer comparison of mutant and wild type performance. Without light reflection at the bottom, even a higher fraction of the incident light intensity would be lost (absorbed at the pond floor) in the mutant culture in comparison to the wild type culture. At high antenna size reductions, the reflective liner could not prevent considerable light losses in mutant monocultures as unabsorbed light was reflected on the pond floor but subsequently could not be fully absorbed in the suspension after which it left the raceway pond reactor at the liquid surface.

Cells in a mixed culture of wild type and antenna size mutants are competing for light energy. Cells with a higher pigmentation have a competitive advantage in this situation, as has recently been verified in competition experiments with phycobilisome-deficient cyanobacteria and the corresponding wild type (Agostoni et al. 2016). However, at the high light exposed volume fraction of the reactor, the most competitive cells, with the highest absorption cross section, are more oversaturated with light energy, resulting in heat dissipation. Stated differently, competitiveness comes at a cost of light use efficiency and productivity. Antenna size mutants grow slower than the wild type in the light limiting part of the reactor which makes them less competitive. The lower competiveness as well as the higher light use efficiency of the mutants were confirmed 
in our simulations. As the photobioreactor productivity of a mutant culture eventually returns to the level of the wild type productivity after a contamination, it can be concluded that the biggest increase in productivity obtained with antenna size reduction also results in the most significant losses upon contamination.

The lower the antenna size reduction, the more competitive the organism becomes and the longer it takes before it is outcompeted by the wild type. Contamination can be caused by wild type cells physically entering the photobioreactor or by mutant cells losing their phenotype after a reverse mutation, after which the natural pigment content is restored. In regard to the contamination level, it became evident that, even in the most optimistic scenario with a minimal contamination of one cell per cubic meter, the effects of contamination are considerable. Moreover, the contamination level is unpredictable and could be higher than the assumed one cell per $\mathrm{m}^{3}$. Therefore it can be stated that, unless novel methods are discovered to increase the competitive power of antenna size mutants, the consequences of wild type contamination severely threaten the potential application of antenna size mutants in large-scale long-term production processes.

We have demonstrated that even a contamination level as low as one cell per cubic meter at the beginning of cultivation can substantially reduce the potential productivity increase that was aimed for by using antenna size mutants. Once a contamination has been detected, thorough cleaning and disinfection of the photobioreactor pond is required with no tolerance for remaining wild type cells or other photosynthetic organisms. This is time consuming and expensive and will probably outbalance the cost reduction that could have been obtained by the application of antenna size reduction.

Would it be possible to increase the competitive power of antenna size mutants? In the study of Flynn et al., the mutants were assumed to have additional properties that made them more competitive. They simulated mutants with an increased growth rate and nutrient use efficiency, a decreased minimum phosphorous and nitrogen quota, a lower maintenance requirement and, under these conditions, the mutant was expected to outcompete the wild type (Flynn et al. 2013). It remains doubtful, however, whether a mutant with all of the aforementioned properties can be designed in practice and remain stable regarding all mutations. For example, in order to obtain a higher biomass specific growth rate, many enzymatic steps must be performed at a higher rate which 
does not appear to be feasible. Another method to improve the competitiveness of the mutant rather than changing its growth characteristics is the application of extreme conditions. This condition should ideally affect the viability of the wild type strain but not that of the mutant. One could think of a built-in resistance of the mutant, for example, tolerance to low/high temperatures or a tolerance to high salt concentrations. However, this would still create a selective environment that rewards invasive species that actually have such a capability (Mooij et al. 2015). Smart techniques are required to enforce the elimination of the wild type while maintaining the mutant cells. Perhaps the wild type's higher sensitivity to light can be exploited as this makes them more prone to photoinhibition while antenna size mutants would only experience photoinhibition at much higher light intensities. Wild type cells possibly have to spend more energy on photosystem II repair processes than antenna size mutants because their higher light absorbance rate will also increase the irreversible damage to the photosystems. To win the competition, the specific growth rate of the mutant should be higher than that of the wild type. At solar noon in Tunisia, the biomass specific growth rate of an $80 \%$ reduced mutant is $1.4 \mathrm{~d}^{-1}$ versus $2.2 \mathrm{~d}^{-1}$ for the wild type according to our growth model. Consequently, severe chronic photoinhibition would be required for the wild type to shift the equilibrium towards the mutant. For Chlorella sorokiniana such severe photoinhibition has not been observed up to light levels of $2000 \mu \mathrm{mol}_{\mathrm{ph}} \mathrm{m}^{-2} \mathrm{~s}^{-1}$ (Cuaresma et al. 2011, Franco et al. 2012) and for this reason photoinhibition was not included in this model.

A more complex dynamic model was presented by Flynn et al. (Flynn 2001, Flynn et al. 2010, Flynn et al. 2013). In accordance with our findings, Flynn and coworkers estimated that under continuous light $\left(1000 \mu \mathrm{mol}_{\mathrm{ph}} \mathrm{m}^{-2} \mathrm{~s}^{-1}\right)$, the use of antenna size mutants will lead to an increased biomass productivity, and the mutants will be outcompeted by the wild type after a contamination (Flynn et al. 2010). The strength of our model is its simplicity and the fact that we only considered mutants with a reduction of the absorption cross section without additional traits. The repetitive light pattern and the lack of seasonal light and temperature variability has the advantage that effects can be unequivocally assigned to the antenna size reduction or the contamination level which was the purpose of this study. The estimations of our model provide a realistic view of the potential of antenna size mutants under large scale outdoor conditions. The 
productivity of our mutants was limited by the inevitable steep light gradient in mass cultures, the low light absorption capacity of mutants, and the fact that the total maintenance requirement can become a critical factor at substantial antenna size reductions. We also included the impact of day/night cycles on microalgal growth. Even though the model includes several simplifications such as that photoinhibition was not included, it is capable of predicting important trends which can be used as input for future research.

\subsection{Conclusions}

A theoretical model was presented to simulate cultivations of antenna size mutants and the competition with their wild type under mass culture conditions. As was expected, the comparison of Tunisia with the Netherlands demonstrated that a substantial increase of productivity is only possible in a location of high irradiance. In addition, a considerable antenna size reduction $(80 \%)$ is required to achieve maximal culture productivity. Mutants with such an antenna size reduction have not been obtained yet in practice. At very high antenna size reduction, it becomes difficult to efficiently absorb all incident light because of the low absorption capacity. In addition, high biomass concentrations are required to absorb all light, which increases cellular maintenances costs. The higher the antenna reduction is, the more sensitive the cultivation process becomes for competition with a full antenna strain. According to the model, antenna size mutants will always lose the competition for light with their wild type and the rate of outcompetition was ascertained to increase with decreasing antenna size and with increasing contamination levels. Based on these findings it can be concluded that the competitiveness of the mutants strains is an important factor that determines whether long-term stable cultivation can be achieved in mass cultures. 


\section{Appendix A: Microalgae growth model}

\section{Module 1: Light distribution in a photobioreactor}

In this algae production model, a hypothetical photobioreactor is studied (Fig. A1) that represents a pond with a constant depth $\left(d_{R}, 0.20 \mathrm{~m}\right)$ and with infinite width and length. The only interface that is considered for reflection and refraction is the air-water interface since the culture is directly in contact with the air at the culture surface. The light intensity on the culture surface must be determined to later predict the light distribution within the culture.

The light intensity gradient in the microalgae culture is calculated in three consecutive steps. First, the light intensity on a horizontal surface on earth is calculated neglecting the effect of atmospheric light absorption (Velds et al. 1992). Second, the absorption of the atmosphere due to its turbidity is incorporated (Velds et al. 1992). In the third step, the refraction and reflection of light passing the air water interphase (Cooper 1969, Kasten et al. 1980, Goldstein 2010) is included to obtain the intensity and directionality of this light that enters the microalgae culture $\left(I_{p h, 0}\right)$. For an extensive description of the equations used to calculate the incident light intensity, refer to Appendix C.

The incident light intensity on a horizontal surface $\left(I_{p h, 0}\right)$ is used as an input in Eq. A1, which is based on Lambert-Beer's law.

$$
I_{p h, \lambda, d o w n}(z)=I_{p h, 0} \cdot E_{n, \lambda} \cdot e^{-\left(a_{X^{\prime}, \lambda} \cdot \lambda_{W T} \cdot X^{\prime} W_{T}+a_{X^{\prime}, \lambda} \cdot \lambda_{M U} \cdot X^{\prime} M U\right) \cdot z \cdot \frac{1}{\cos \theta_{Z}^{\prime}}}
$$

$E_{n, \lambda}\left(\mathrm{nm}^{-1}\right)$ represents the relative sunlight distribution for the range of photosynthetic active radiation (see Appendix E for values). The absorption cross sections of the wild type strain and the mutant are denoted by $a_{X^{\prime}, \lambda_{W T}}$ and $a_{X^{\prime}, \lambda_{M U}}$, respectively. The length of the light path in the culture is influenced by the angle between the refracted sunrays and the perpendicular to the culture surface (angle $\theta_{z}^{\prime}$, Fig. A1). In the model, the position $z$ is multiplied with the enhancing factor, the inverse of the cosine of $\theta_{z}{ }^{\prime}$, to correct for the lengthening of the optical path at increasing $\theta_{z}^{\prime}$. An increasing optical path increases the chance of light absorption and results in a steeper light gradient in the $z$-direction. 
To minimize light losses at the bottom of the pond and to obtain a fairer comparison between wild type and mutant culture productivity, the pond floor was simulated to be covered with a white reflective ground cover. This cover diffusively reflects $80 \%$ (Meinhold et al. 2010) of the light at the bottom back into the culture. Moreover, the application of white liners is also common practice in the raceway pond construction. The addition of a reflective liner results in a second light flux $I_{p h, \lambda, u p}(z)$ from the pond floor back to the culture surface. The light intensity in the culture only resulting from this flux is described by:

$$
\left.I_{p h, \lambda, u p}(z)=I_{p h, \lambda, \text { down }}(z) \cdot f_{R} \cdot e^{-\left(a_{X^{\prime}, \lambda} \cdot{ }_{W T} \cdot X^{\prime}{ }_{W T}+a_{X^{\prime}, \lambda_{M U}} \cdot X^{\prime} M U\right.}\right) \cdot\left(d_{R}-z\right) \cdot \frac{1}{\cos \theta_{Z, d i f^{\prime}}}
$$

with $d_{R}$ being the reactor depth and $f_{R}$ being the fraction of reflected light. The inverse of the cosine of $\theta_{z, d i f}$ is the factor with which the reflected optical light path is extended. The diffuse reflection corresponds to an average angle $\theta_{z, \text { dif }}$ of $60^{\circ}$.

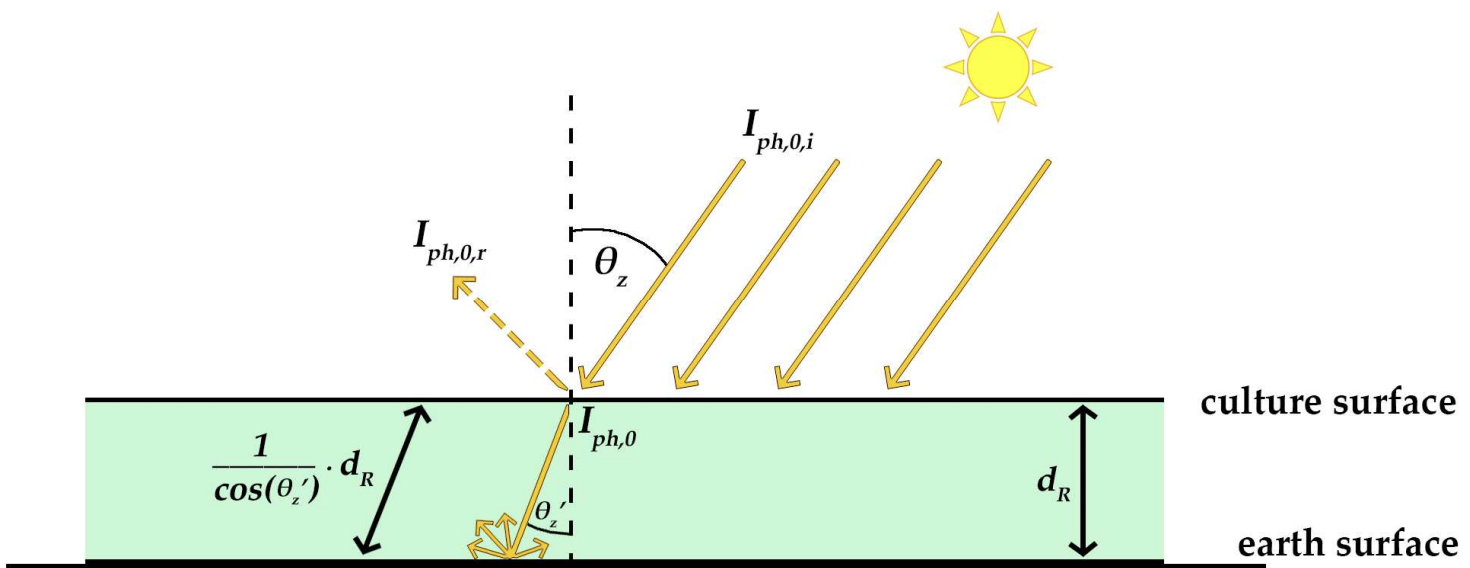

Fig. A1 Schematic overview of the raceway pond photobioreactor. The sunlight hits the culture surface with the light intensity $I_{p h, 0, i}$ at the zenith angle $\theta_{z}$. The light is partly reflected $\left(I_{p h, 0, r}\right)$ and partly transmitted into the culture $\left(I_{p h, 0}\right)$. The refraction at the culture surface leads to the refracted zenith angle $\theta_{z}{ }^{\prime}$. The light reaching the bottom of the pond is, in part (80\%), diffusively reflected back into the culture by a white ground cover.

\section{Module 2: Photosynthetic sugar production}

To model the competition for light in a culture of an antenna size mutant contaminated 
with its wild type, the specific light absorption rates of both strains must be distinguished. The specific light absorption rates then allow the calculation of specific sugar production rates for each strain. The sugar production rate $\left(q_{S_{i}}\right)$ of one organism (indicated by index i) in a culture containing wild type and mutant cells can be described according to the hyperbolic tangent model of Jassby and Platt (Jassby et al. 1976):

$$
q_{S_{i}}=q_{S}^{\max } \cdot \tanh \left(\frac{q_{p h, i} \cdot Y_{S / p h, m}}{q_{S}^{\max }}\right)
$$

The calculation of the sugar production rate is based on the photon absorption rate $\left(q_{p h, \lambda, i}\right)$, the maximum yield of sugar on photons $\left(Y_{S / p h, m}\right)$, and the maximum sugar production rate $\left(q_{S}^{\max }\right)$, (see Table E3 of Appendix E for values). The biomass specific light absorption rate $\left(q_{p h, \lambda, i}\right)$ can be described for wild type $(i=W T)$ and mutant $(i=M U)$ in one photobioreactor simultaneously by utilizing Lambert-Beer's law:

$$
\begin{aligned}
& \frac{d I_{p h, \lambda, \text { down }}}{d z}=I_{p h, \lambda, \text { down }}(z) \cdot\left(a_{X^{\prime}{ }_{W T}, \lambda} \cdot X^{\prime}{ }_{W T}+a_{X^{\prime}{ }_{M U}, \lambda} \cdot X^{\prime}{ }_{M U}\right) \cdot \frac{1}{\cos \theta_{z}{ }^{\prime}} \\
& \frac{d I_{p h, \lambda, u p}}{d z}=I_{p h, \lambda, u p}(z) \cdot\left(a_{X^{\prime}{ }_{W T}, \lambda} \cdot X^{\prime}{ }_{W T}+a_{X^{\prime}{ }_{M U}, \lambda} \cdot X^{\prime}{ }_{M U}\right) \cdot \frac{1}{\cos \theta_{z, \text { diff }}{ }^{\prime}} \\
& q_{p h, \lambda, i}=\left(-\frac{d I_{p h, \lambda, d o w n}}{d z}+\frac{d I_{p h, \lambda, u p}}{d z}\right) \cdot \frac{1}{X^{\prime}{ }_{i}} \cdot \frac{a_{X^{\prime}} \cdot X^{\prime}{ }_{i}}{a_{X^{\prime}{ }_{W T}, \lambda} \cdot X^{\prime}{ }_{W T}+a_{X^{\prime}{ }_{M U, \lambda} \lambda} \cdot X^{\prime}{ }_{M U}}
\end{aligned}
$$

with the light gradients $\frac{d I_{p h, \lambda, d o w n}}{d z}$ and $\frac{d I_{p h, \lambda, u p}}{d z}$, the concentration of functional biomass $\left(X^{\prime}{ }_{i}\right)$, the absorption cross section $\left(a_{X^{\prime}}{ }_{i}, \lambda\right)$, the local light intensity $\left(I_{p h, \lambda}(z)\right)$, and the factor $\left(\frac{1}{\cos \theta_{z}^{\prime}}\right)$ that corrects the light path for its deviation from the $z$ direction. Apparently, the light regime in the reactor (Eq. A4 and Eq. A5) is dictated by the total light absorption capacity of both the wild type and mutant strain. The biomass specific light absorption rates $\left(q_{p h, i}\right)$ (Eq. A6) depend on the absorbed fraction of this total light energy which is based on the light absorption capacity and biomass concentration of each strain. Eq. A4-A6 are combined to give: 


$$
q_{p h, i, \lambda}(z)=a_{X^{\prime}, \lambda_{i}} \cdot\left(I_{p h, \lambda, \text { down }}(z) \cdot \frac{1}{\cos \theta_{z}^{\prime}}+I_{p h, \lambda, u p}(z) \cdot \frac{1}{\cos \theta_{z, \text { diff }}{ }^{\prime}}\right)
$$

By combining Eq. A3 and Eq. A7 and adding the wavelength dependency of $Y_{S / p h, m}$, the specific sugar production rates of the mutant and wild type were obtained:

$$
q_{S_{i}}(z)=q_{S}^{\max } \cdot \tanh \left(\frac{\sum_{\lambda=400}^{\lambda=700}\left(q_{p h, i, \lambda}(z) \cdot Y_{S / p h, m, \lambda}\right) \cdot \Delta \lambda}{q_{S}^{\max }}\right)
$$

The specific sugar production rates (Eq. A8) were concurrently simulated in one photobioreactor for both strains to model the competition for light. The produced sugar is partly used for maintenance related processes, partly stored in the sugar reserve pool, and partly used for production of new functional biomass.

\section{Module 3: Partitioning of produced sugar during the diurnal cycle}

The carbohydrate starch is an important energy reserve for the growth of microalgae in day/night cycles (Ogbonna et al. 1996, Sharkey 2015). Synthetized starch is stored as semi-crystalline granules (Sonnewald et al. 2013) and can be oxidized by the cell to gain energy and reduce carbon upon demand (Lacour et al. 2012). The accumulation of starch occurs during the day when it serves as an energy sink while the degradation primarily occurs during the night to provide energy and carbon (Post et al. 1985, Sukenik et al. 1990, Fábregas et al. 2002, Lacour et al. 2012).

A mechanism of carbon partitioning during the diurnal cycle was developed. This mechanism includes partitioning of the photosynthetically produced sugar to the functional biomass and the sugar reserve pool. The longer the night period and the lower the current sugar reserves, the more sugar that is stored in the sugar pool during the day to accumulate sufficient reserves for the entire night period. Additionally, sugar from the sugar pool is converted into functional biomass at a rate that depends on the sugar availability. The conversion rate will automatically be re-adjusted in such a way that it would be stable until the end of the night if no additional sugar is generated. This leads to a minimum sugar fraction in the total biomass at the end of the night. 
The production rates of functional biomass and sugar are described in two differential equations (Eq. A9 and Eq. A10) which are applied on both strains simultaneously:

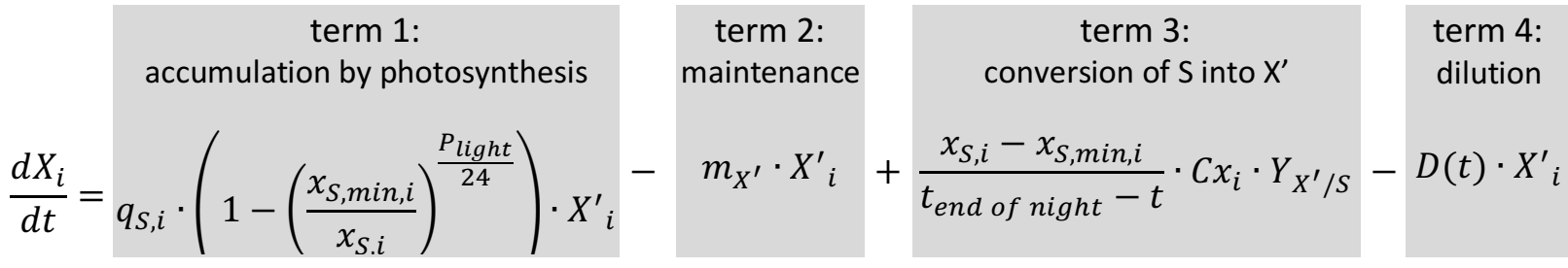

$$
\begin{aligned}
& \text { term 1: } \\
& \text { accumulation by photosynthesis } \\
& \frac{d S_{i}}{d t}=\quad q_{S, i} \cdot\left(\frac{x_{S, \min , i}}{x_{S, i}}\right)^{\frac{P_{\text {light }}}{24}} \cdot X^{\prime}{ }_{i}-m_{S} \cdot X^{\prime}{ }_{i}-\frac{x_{S, i}-x_{S, \min , i}}{t_{\text {end of night }}-t} \cdot C x_{i}-D(t) \cdot S_{i}
\end{aligned}
$$

With:

$m_{X^{\prime}}=0$ for $x_{S, i}>x_{S, \min , i}$

$m_{S}=0$ for $x_{S, i}=x_{S, \min , i}$

The biomass accumulation rate (Eq. A9) and the sugar accumulation rate (Eq. A10) depend, among others, on the total biomass concentration $\left(C x_{i}\right)$ and the concentration of functional biomass $\left(X^{\prime}{ }_{i}\right)$ and sugar $\left(S_{i}\right) . m_{X^{\prime}}$ is the maintenance requirement $\left(\right.$ mol $_{X^{\prime}}$. $\left.\operatorname{mol}_{X^{\prime}}{ }^{-1} \cdot s^{-1}\right)$ covered by degradation of functional biomass. The molar fraction of sugar in biomass is given as $x_{S, i}=S_{i} /\left(X^{\prime}{ }_{i}+S_{i}\right)$. The index $i$ indicates that the equations need to be separately applied on the two strains, wild type $(i=W T)$ and mutant $(i=M U)$. The cultivation time, $\mathrm{t}(0-24$ hours $)$, is the local solar time.

The differential equations describing the accumulation of functional biomass and sugars in the photobioreactor (Eq. A9 and Eq. A10) both include four terms. The first term depends on the sugar production rate (Eq. A8) and, therefore, the amount of absorbed light. Carbon fixated and reduced by photosynthesis partitions between the synthesis of functional biomass and the sugar pool. The partitioning depends on the sugar fraction of biomass $\left(x_{S, i}\right)$ at the investigated time point and the duration (hours) of the light period $\left(P_{\text {light }}\right)$ in one day/night cycle of 24 hours. The shorter the light period, the more sugar 
reserves are needed during the night to cover the maintenance requirement and support the generation of functional biomass in the dark (Lacour et al. 2012). To restore the sugar reserves for the consumption during the night, low sugar fractions in the biomass lead to a high allocation rate of photosynthetically produced sugar to the sugar pool. This behavior is simulated by a Droop based equation (Gibson et al. 2008).

The second term of the equations Eq. A9 and Eq. A10 describes the specific maintenance requirement of the functional biomass. The maintenance requirement $\left(m_{S}\right)$ is primarily covered by the respiration of sugar. Sufficient sugar reserves to fulfill maintenance requirements are ensured by the sugar accumulation mechanism. However, in the exceptional case that the sugar reserves are too low $\left(x_{S, i} \leq x_{S, \min , i}\right)$, the only way to fulfill maintenance requirements $\left(m_{X^{\prime}}\right)$ is by the degradation of functional biomass, as shown by the second term of Eq. A9.

Independent from light conditions, the third term ensures the conversion of sugar to functional biomass. This rate is linear during the night and results in the minimum sugar fraction $x_{S, \min , i}$ at the end of the night. Based on starch turnover (Klein 1987), a minimum production rate of functional biomass is ensured over the entire course of the day as soon as sugar has been accumulated. First, the released sugar is used for maintenance related processes, and the remaining sugar subsequently enters the functional biomass production. This way, functional biomass is produced during the night at the expense of the sugar pool. Sugar is employed for the production of functional biomass according to the functional biomass yield on sugar $Y_{X^{\prime} / S}$. This yield has a value less than 1 , reflecting the fact that a significant portion of the sugar needs to be respired to retrieve sufficient energy in the form of ATP to incorporate the remaining sugar into functional biomass. The last term of the differential equations describes the removal of functional biomass and the sugar reserves by the applied system dilution, as the raceway pond reactor is operated as a chemostat. Dilution is only applied during daylight hours. 


\section{Appendix B: Equations to calculate location and wavelength-specific light distribution}

\section{Solar power and sunlight angle on a horizontal surface}

In this calculation, the effect of the turbidity of the atmosphere is first neglected, however, the predicted solar power is later corrected for the contribution of atmospheric absorption. The solar power on a horizontal surface $\left(E_{e}\right)$ depends on the intensity of sunlight reaching the earth and the zenith angle $\left(\theta_{z}\right.$, Eq. A12). The latter is the angle between the sunrays and the perpendicular to the earth's surface (Fig. ).

$$
E_{e}=S_{0} \cdot \frac{\bar{R}^{2}}{R^{2}} \cdot \cos \theta_{z} \approx S_{0} \cdot\left(1+0.033 \cdot \cos \left(\frac{360}{365} \cdot(d-1)\right)\right) \cdot \cos \theta_{z}
$$

In Eq. A11, all light is assumed to pass from the center of the sun to the earth neglecting effects of the atmosphere. The yearly averaged solar power on earth is described by the solar constant $\left(S_{0}, 1367 \mathrm{~W} \cdot \mathrm{m}^{-2}\right.$ (Fröhlich et al. 1981)). The solar power varies with the factor $\frac{\bar{R}^{2}}{R^{2}}$ over the year due to the ellipsoidal shape of the earth's orbit (Duffie et al. 1980). The daytime and the location dependency of the insolation are both described by the zenith angle:

$$
\theta_{z}=\cos ^{-1}(\sin \varphi \cdot \sin \delta+\cos \varphi \cdot \cos \delta \cdot \cos \omega)
$$

The latitude $(\varphi)$ is directly named in Eq. A12 while the longitude is indirectly included through the hour angle $\left(\omega=15 \cdot\left(t_{\text {solar }}-12\right)\left[{ }^{\circ}\right]\right)$. The position of the sun is highest at $0^{\circ}$ or $t_{\text {solar }}=12 \mathrm{~h}$. The tilt of the earth relative to the sun throughout the year is described by the solar declination $\left(\delta=23.45 \cdot \sin \left(\frac{360 \cdot(284+d)}{365}\right)\left[^{\circ}\right]\right)$.

The solar power neglecting the atmosphere $\left(E_{e}\right)$ can now be corrected for atmospheric absorption. As a simplification, the total global irradiation is considered as direct light even though it includes a diffuse part. Thus, the solar power reaching the earth at a specific location $\left(G_{\text {clear }}\right)$ and with a clear sky is described as:

$$
G_{\text {clear }}=0.84 \cdot E_{e} \cdot e^{\left(-\frac{0.027 T_{L}}{\cos \theta_{Z}}\right)}
$$

The turbidity factor of the atmosphere by Linke $\left(T_{L}\right)$ has been determined experimentally. Under a clear sky, the factor only depends on the time during the year and the location on earth (Velds et al. 1992, Chaâbane et al. 2004). The calculated 
surface-specific solar power using the turbidity factor is converted into the light intensity. The light intensity in the range of the photosynthetic active radiation (PAR, 400 to $700 \mathrm{~nm}$ ) is calculated using a conversion factor according to ASTM G173-03 Reference Spectra derived from SMARTS v. 2.9.2:

$$
I_{p h, 0, i}=G_{\text {clear }} \cdot 1.982 \cdot 10^{-6} \frac{\mathrm{mol}_{\mathrm{ph}, \mathrm{PAR}}}{\mathrm{J}_{\text {solar }}}
$$

\section{Light gradient resulting from refraction and reflection on the culture surface}

The sunlight with the intensity $I_{p h, 0, i}$ hits the culture surface which is assumed to have the same properties as a water surface. Two effects occur there simultaneously; light is refracted and reflected. Due to refraction, the zenith angle $\left(\theta_{z}\right)$ changes according to Snell's law to $\theta_{z}{ }^{\prime}$. Thus, the factor $\left(\frac{1}{\cos \left(\theta_{z^{\prime}}\right)}\right)$ describing the light path dependent on the culture depth reads as:

$$
\frac{1}{\cos \left(\theta_{z}{ }^{\prime}\right)}=\frac{1}{\cos \left(\sin ^{-1}\left(\frac{n_{\text {air }}}{n_{\text {water }}} \cdot \sin \theta_{z}\right)\right)}
$$

The enhancing factor (Eq. A15) is based on the refractive indexes of air $\left(n_{\text {air }}\right)$ and water $\left(n_{\text {water }}\right)$ and trigonometric equations. This expression is used to relate the culture depth to the light path. By applying Lambert-Beer's, this affords calculation of the light distribution inside the culture. The incident light intensity is reduced compared to the description in Eq. A14 since light is partly reflected by the culture surface and partly transmitted into the culture. The reflected light intensity is subtracted from the light intensity hitting the culture surface according to Fresnel's equations:

$$
I_{p h, 0}=I_{p h, 0, i}-\frac{1}{2} \cdot\left(R_{s}+R_{p}\right) \cdot I_{p h, 0, i}
$$

with the reflectivity of light polarized in the plane of incidence $R_{S}=\left(\frac{n_{\text {air }} \cdot \cos \theta_{z^{\prime}}-n_{\text {water }} \cdot \cos \theta_{z}}{n_{\text {air }} \cdot \cos \theta_{z^{\prime}}+n_{\text {water }} \cdot \cos \theta_{z}}\right)^{2}$ and perpendicular to the plane of incidence $R_{p}=$ $\left(\frac{n_{\text {water }} \cdot \cos \theta_{z^{\prime}}-n_{\text {air }} \cdot \cos \theta_{z}}{n_{\text {water }} \cdot \cos \theta_{z^{\prime}}+n_{\text {air }} \cdot \cos \theta_{z}}\right)^{2}$. 


\section{Appendix C: Light patterns}

The irradiance and the light angle were simulated for a sunny location (Sidi Bou Said, Tunisia) and a location with a higher cloudage (De Bilt, the Netherlands). Measurements showed a global insolation of $16.67 \mathrm{MJ} \mathrm{m}^{-2} \mathrm{~d}^{-1}$ in mid-July (1971-1986) in the Netherlands (Velds et al. 1992). This insolation corresponds to $62 \%$ of the predicted insolation under a clear sky. Compared to that, in Tunisia, the average insolation in July ( $>10$ years) is significantly higher (26.64 $\mathrm{MJ} \mathrm{m}^{-2} \mathrm{~d}^{-1}$ over $>10$ years) (Alnaser et al. 2004). This value is $5 \%$ lower than the predicted insolation in Tunisia. While the low cloudage in Tunisia was neglected in the simulations, a constant cloudage of $40 \%$ was assumed in the Netherlands. All light including the diffuse part was assumed to be direct to simplify the simulations. The resulting light patterns for the chosen summer day in July that was used in all simulations are presented in Fig. A2.

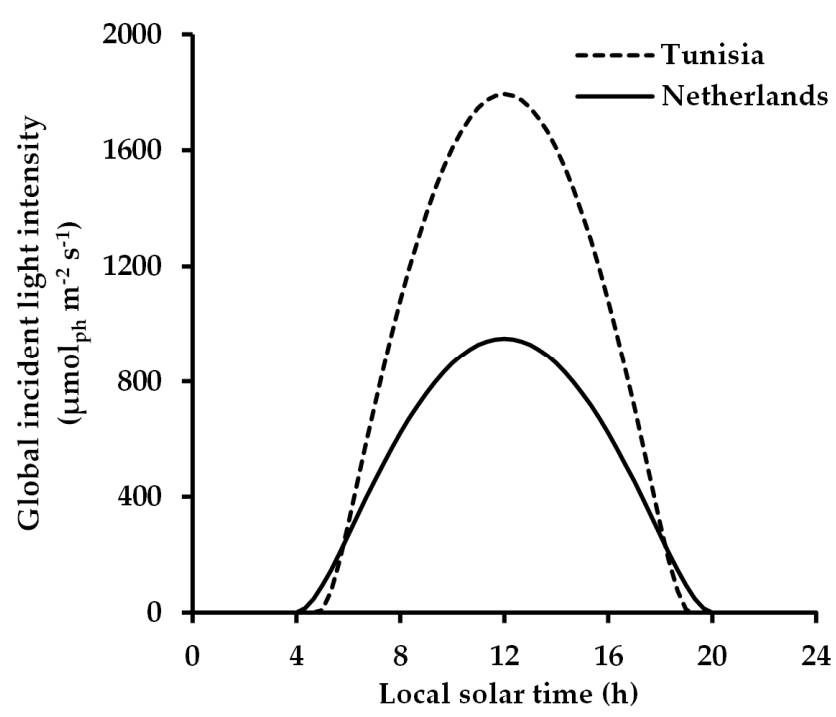

Fig. A2 Global incident light intensity in the PAR range on a horizontal surface in Tunisia and the Netherlands in the course of one day/night cycle. The light intensity is derived from the light model as described in Appendix B. No cloud cover is assumed in Tunisia while the light intensity is evenly reduced by $40 \%$ in the Netherlands to simulate the cloud cover. 


\section{Appendix D: Dilution optimization procedure}

The dilution rate was separately optimized for the areal biomass productivity of each strain. The optimization of the constant dilution rate during the day was performed employing a controlled random search algorithm. The boundaries for the dilution rate were set to zero and the maximum growth rate to $\left(0.27 \mathrm{~h}^{-1}\right)$. Within these boundaries, 50 random values for the dilution rate were drawn. A cultivation period of 15 days was simulated for each dilution rate. The average productivity was calculated for the last 24 hours. All dilution rates and the corresponding productivities were recorded on a list with 50 data pairs. Stepwise, the dilution rates and productivities in the list were replaced with data pairs containing higher productivities. First, four dilution rates $\left(D_{r, 1}\right.$ to $\left.D_{r, 4}\right)$ were randomly drawn and combined with the last dilution rate in the list $\left(D_{\text {end }}\right)$ to return a new value $\left(D_{\text {new }}\right.$, Eq. A17).

$$
D_{\text {new }}=2 \cdot\left(\frac{\sum_{i=1}^{i=4} D_{r, i}}{4}\right)-D_{\text {end }}
$$

Second, the same simulation as described above followed for the new dilution rate if the boundary conditions were still met. Otherwise, a new value was generated and the simulation was performed with the first value within the boundary conditions. Third, the productivity resulting from this simulation was compared to the lowest productivity in the list. The data pair with the lowest productivity was replaced if it the new productivity was higher. The new data pair was discarded if the new productivity was lower. By repeating this pattern, a dense data set around the optimal dilution rate was generated (See Fig. A3 for an example). The dilution rate corresponding to the maximum areal biomass productivity was used for all simulations with the respective strain.

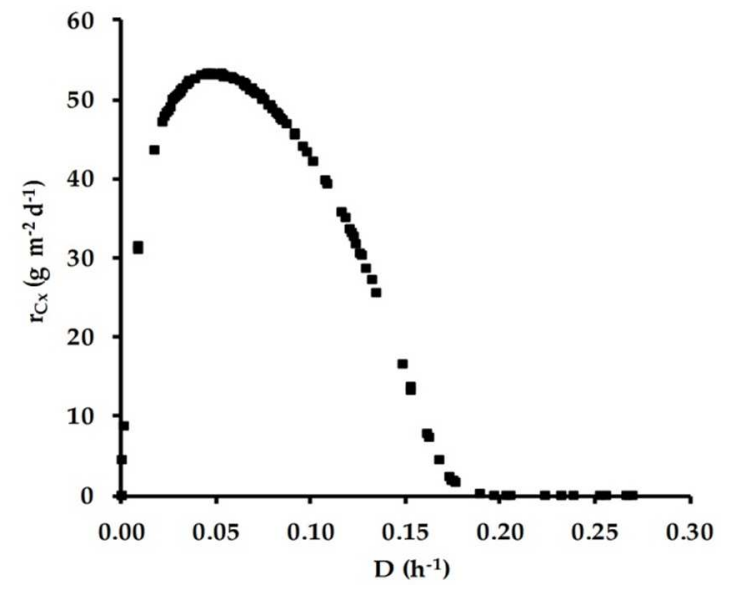

Fig. A3 Areal productivity versus dilution rate of a mutant that is $80 \%$ reduced in $\boldsymbol{a}_{\boldsymbol{X}}$ simulated for cultivation in Tunisia. Dilution only occurred during light hours at a constant rate. During the night, there was no dilution. The daily light intensity was $55.8 \mathrm{~mol}_{p h} \mathrm{~m}^{-2} \mathrm{~d}^{-1}$. 


\section{Appendix E: Model variables and parameters}

Table E1 Overview of location and time dependent variables and parameters used for the derivation of the light pattern (Appendix B).

\begin{tabular}{|c|c|c|}
\hline Symbol & Description & Unit \\
\hline NL & Location in the Netherlands, town: De Bilt & - \\
\hline TU & Location in North Tunisia, town: Sidi Bou Said & - \\
\hline$t$ & Cultivation time with $t_{0}=8$ am local solar time & $\mathrm{h}$ \\
\hline$d$ & Number of the day in the year & - \\
\hline$t_{\text {solar }}$ & Local solar time, position of the sun is highest at $0^{\circ}$ or $\boldsymbol{t}_{\text {solar }}=12 \mathrm{am}$ & $\mathrm{h}$ \\
\hline$P_{\text {light }}$ & Duration of the light period in one day/night cycle & $\mathrm{h}$ \\
\hline$P A R$ & Photosynthetic active radiation (spectrum of 400 to $700 \mathrm{~nm}$ ) & - \\
\hline$\lambda$ & Wavelength & $\mathrm{nm}$ \\
\hline $\boldsymbol{E}_{e}$ & Solar power on the horizontal earth surface neglecting effects of the atmosphere & $\frac{\mathrm{W}}{\mathrm{m}^{2}}$ \\
\hline$G_{\text {clear }}$ & $\begin{array}{l}\text { Solar power reaching the earth on a specific location including effects of the } \\
\text { atmosphere }\end{array}$ & $\frac{\mathrm{W}}{\mathrm{m}^{2}}$ \\
\hline$I_{p h, 0, i}$ & Global light intensity that hits the earth surface before light is reflected & $\frac{\mathrm{mol}_{\mathrm{ph}}}{\mathrm{m}^{2} \cdot \mathrm{s}}$ \\
\hline$I_{p h, 0}$ & $\begin{array}{l}\text { Global light intensity on the horizontal culture surface that is transmitted into the } \\
\text { culture according to Fresnel's equations }\end{array}$ & $\frac{\mathrm{mol}_{\mathrm{ph}}}{\mathrm{m}^{2} \cdot \mathrm{s}}$ \\
\hline$I_{p h, 0, r}$ & Global light intensity that is reflected at the reactor surface. & $\frac{\mathrm{mol}_{\mathrm{ph}}}{\mathrm{m}^{2} \cdot \mathrm{s}}$ \\
\hline$I_{p h, \lambda, d o w n}(z)$ & $\begin{array}{l}\text { Wavelength-specific light intensity at every position } z \text { without the addition of light } \\
\text { that is reflected at the pond floor. }\end{array}$ & $\frac{\mathrm{mol}_{\mathrm{ph}}}{\mathrm{m}^{2} \cdot \mathrm{s}}$ \\
\hline$I_{p h, \lambda, u p}(\mathbf{z})$ & $\begin{array}{l}\text { Wavelength-specific light intensity at every position } z \text { limited to light that is } \\
\text { reflected at the bottom of the pond floor. }\end{array}$ & $\frac{\mathrm{mol}_{\mathrm{ph}}}{\mathrm{m}^{2} \cdot \mathrm{s}}$ \\
\hline$\frac{1}{\cos \left(\theta_{z}{ }^{\prime}\right)}$ & Enhancement factor correlating the light path to the reactor depth & - \\
\hline $\boldsymbol{\theta}_{\mathrm{z}}$ & Zenith angle, the angle between sunrays and the perpendicular to the earth surface & $\circ$ \\
\hline $\boldsymbol{\theta}_{\mathrm{z}}{ }^{\prime}$ & Refracted zenith angle, the zenith angle after refraction according to Snell's law & $\circ$ \\
\hline$\theta_{z, \text { diff }}$ & $\begin{array}{l}\text { Refracted zenith angle, the zenith angle after refraction at the bottom of the pond } \\
\text { photobioreactor. }\end{array}$ & \\
\hline $\boldsymbol{\delta}$ & Solar declination, tilt of the earth relative to the sun & $\circ$ \\
\hline$\overline{\boldsymbol{R}}$ & Average distance between the earth and sun over the year & - \\
\hline $\boldsymbol{R}$ & Average distance between the earth and sun over the day & - \\
\hline $\boldsymbol{R}_{\mathrm{s}}$ & Reflectivity of light polarized in the plane of incidence & - \\
\hline$R_{p}$ & Reflectivity of light polarized perpendicular to the plane of incidence & - \\
\hline
\end{tabular}




\begin{tabular}{|c|c|c|c|c|}
\hline Symbol & Description & & & Unit \\
\hline Parameter & Description & Value & Unit & Ref. \\
\hline$S_{0}$ & Solar constant & 1367.0 & $\frac{\mathrm{W}}{\mathrm{m}^{2}}$ & $\begin{array}{l}\text { (Fröhlich et al. } \\
\text { 1981) }\end{array}$ \\
\hline$T_{L}$ & $\begin{array}{l}\text { Turbidity coefficient by Linke, TU: averaged } \\
\text { from daily values in mid of July in, NL: July } \\
15^{\text {th }} \text { in De Bilt }\end{array}$ & $\begin{array}{l}5.9[\mathrm{NL}] \\
5.5[\mathrm{TU}]\end{array}$ & - & $\begin{array}{l}\text { (Velds et al. 1992, } \\
\text { Chaâbane et al. } \\
\text { 2004) }\end{array}$ \\
\hline$\varphi$ & Latitude & $\begin{array}{l}52.117[\mathrm{NL}] \\
36.867[\mathrm{TU}]\end{array}$ & - & \\
\hline$f_{R}$ & $\begin{array}{l}\text { Fraction of reflected light of reflective ground } \\
\text { cover. }\end{array}$ & 0.8 & - & $\begin{array}{l}\text { (Meinhold et al. } \\
\text { 2010) }\end{array}$ \\
\hline
\end{tabular}

Table E2 Overview of time dependent variables describing the sugar production rate and carbon partitioning and the productivity. The index $i$ refers to the application of the parameter on both strains in the reactor, the mutant and the wild type.

\begin{tabular}{|c|c|c|}
\hline Symbol & Description & Unit \\
\hline$q p h_{i}$ & Biomass specific photon absorption rate & $\frac{\mathrm{mol}_{\mathrm{ph}}}{\mathrm{mol}_{\mathrm{X}^{\prime}} \cdot \mathrm{s}}$ \\
\hline$q_{S_{i}}$ & Biomass specific sugar production rate & $\frac{\mathrm{mol}_{\mathrm{S}}}{\mathrm{mol}_{\mathrm{X}^{\prime}} \cdot \mathrm{s}}$ \\
\hline$\frac{d X^{\prime}{ }_{i}}{d t}$ & Accumulation rate of functional biomass & $\frac{\mathrm{mol}_{\mathrm{X}^{\prime}}}{\mathrm{s} \cdot \mathrm{m}^{3}}$ \\
\hline$\frac{d S_{i}}{d t}$ & Accumulation rate of sugar stored as starch & $\frac{\mathrm{mol}_{\mathrm{s}}}{\mathrm{s} \cdot \mathrm{m}^{3}}$ \\
\hline$C x_{i}$ & Total molar biomass concentration in the reactor (normalized to $1 \mathrm{~mol}$ carbon) & $\frac{\mathrm{mol}_{\mathrm{Cx}}}{\mathrm{m}^{3}}$ \\
\hline$Y_{C x / p h}$ & The biomass yield on incident light energy & $\frac{\mathrm{g}_{\mathrm{Cx}}}{\mathrm{mol}_{\mathrm{ph}}}$ \\
\hline $\boldsymbol{X}^{\prime}{ }_{i}$ & Molar concentration of functional biomass (normalized to 1 mol carbon) & $\frac{\mathrm{mol}_{\mathrm{x}^{\prime}}}{\mathrm{m}^{3}}$ \\
\hline$S_{i}$ & Molar concentration of sugar stored as starch (normalized to 1 mol carbon) & $\frac{\mathrm{mol}_{\mathrm{S}}}{\mathrm{m}^{3}}$ \\
\hline$x_{S, i}$ & Molar fraction of sugar in the total biomass & $\frac{\mathrm{mol}_{\mathrm{S}}}{\mathrm{mol}_{\mathrm{Cx}}}$ \\
\hline$r_{C x}$ & Areal biomass productivity & $\frac{g_{C x}}{m^{2} \cdot d}$ \\
\hline
\end{tabular}


Table E3 Overview of model parameters related to the organism, substances, or the light and the reactor. Organism specific parameters are either originating from C. sorokiniana or generally assumed for green algae. The index $i$ refers to the application of the parameter on both strains in the reactor, the mutant and the wild type.

\begin{tabular}{|c|c|c|c|c|c|}
\hline Parameter & Value & Unit & Description & Origin & Ref. \\
\hline$\mu_{\mathrm{m}}$ & 0.27 & $\mathrm{~h}^{-1}$ & Maximum biomass specific growth rate ${ }^{1}$ & C. sorokiniana & $\begin{array}{r}\text { (Blanken et } \\
\text { al. 2016) }\end{array}$ \\
\hline $\boldsymbol{m}_{S}$ & $2.69 \cdot 10^{-6}$ & $\frac{\mathrm{mol}_{\mathrm{S}}}{\mathrm{mol}_{\mathrm{X}^{\prime}} \cdot \mathrm{s}}$ & $\begin{array}{l}\text { Biomass specific maintenance rate } \\
\text { normalized to functional biomass }\end{array}$ & C. sorokiniana & $\begin{array}{r}\text { (Blanken et } \\
\text { al. 2016) }\end{array}$ \\
\hline$m_{X^{\prime}}$ & $2.41 \cdot 10^{-6}$ & $\frac{\mathrm{mol}_{\mathrm{X}^{\prime}}}{\mathrm{mol}_{\mathrm{X}^{\prime}} \cdot \mathrm{s}}$ & $\begin{array}{l}\text { Biomass specific maintenance rate } \\
\text { normalized to functional biomass }\end{array}$ & & $\begin{array}{r}\text { (Blanken et } \\
\text { al. 2016) }\end{array}$ \\
\hline$Y_{X^{\prime} / S}$ & 0.56 & $\frac{\mathrm{mol}_{\mathrm{X}^{\prime}}}{\mathrm{mol}_{\mathrm{S}}}$ & $\begin{array}{l}\text { Functional biomass yield on 3-carbon } \\
\text { sugar using ammonia as nitrogen source }\end{array}$ & C. sorokiniana & $\begin{array}{r}\text { (Blanken et } \\
\text { al. 2016) }\end{array}$ \\
\hline$q_{S}^{\max }$ & $1.21 \cdot 10^{-4}$ & $\frac{\mathrm{mol}_{\mathrm{S}}}{\mathrm{mol}_{\mathrm{X}^{\prime}} \cdot \mathrm{s}}$ & $\begin{array}{l}\text { Maximum functional biomass specific } \\
\text { sugar production rate, } \\
\mu_{m}=\left(q_{S}^{\max }-m_{S}\right) \cdot Y_{\mathrm{X}^{\prime} / S}\end{array}$ & C. sorokiniana & \\
\hline$x_{S, \min , i}$ & 0.07 & $\frac{\mathrm{mol}_{\mathrm{S}}}{\mathrm{mol}_{\mathrm{Cx}}}$ & $\begin{array}{l}\text { Minimum sugar content } 0.08 \frac{g_{S}}{g_{C x}} \text { in total } \\
\text { biomass }\end{array}$ & C. sorokiniana & $\begin{array}{r}\text { (Blanken et } \\
\text { al. 2016) }\end{array}$ \\
\hline $\mathbf{a}_{\mathbf{X}^{\prime}, \lambda, \mathbf{i}}$ & $\begin{array}{c}\text { see } \\
\text { table E4 }\end{array}$ & $\frac{\mathrm{m}^{2}}{\mathrm{~mol}_{\mathrm{X}^{\prime}} \cdot \mathrm{nm}}$ & $\begin{array}{l}\text { Wavelength-specific absorption cross } \\
\text { section, value for mutant as a percentage } \\
\text { of wild type }\end{array}$ & C. sorokiniana & \\
\hline$Y_{S / p h, m, \lambda}$ & $\begin{array}{c}\text { see } \\
\text { table E4 }\end{array}$ & $\frac{\mathrm{mol}_{\mathrm{S}}}{\mathrm{mol}_{\mathrm{ph}} \cdot \mathrm{nm}}$ & $\begin{array}{l}\text { Maximum wavelength dependent yield of } \\
\text { sugar on photons }\end{array}$ & $\begin{array}{l}\text { Cyanobacteria, } \\
\text { green algae }\end{array}$ & \\
\hline$M_{X^{\prime}}$ & 24 & $\frac{\mathrm{g}_{\mathrm{x}^{\prime}}}{\mathrm{mol}_{\mathrm{X}^{\prime}}}$ & $\begin{array}{l}\text { Biomass dry weight to C-mol conversion } \\
\text { factor for functional biomass. }\end{array}$ & & \\
\hline$M_{S}$ & 27 & $\frac{\mathrm{g}_{\mathrm{S}}}{\mathrm{mol}_{\mathrm{S}}}$ & $\begin{array}{l}\text { Molecular weight of a polysaccharides of } \\
\text { glucose normalized to } 1 \text { mol carbon }\end{array}$ & & $\begin{array}{l}\text { IFA GESTIS- } \\
\text { Stoffdatenbank }\end{array}$ \\
\hline $\boldsymbol{n}_{\text {air }}$ & 1.00 & - & Refractive index of air & & $\begin{array}{r}\text { (Denny } \\
1993 \text { ) }\end{array}$ \\
\hline$n_{\text {water }}$ & 1.33 & - & Refractive index of water & & $\begin{array}{r}\text { (Denny } \\
1993)\end{array}$ \\
\hline$z$ & & $\mathrm{~m}$ & Position in the reactor & & \\
\hline$d_{R}$ & 0.20 & $\mathrm{~m}$ & Reactor depth & & \\
\hline $\boldsymbol{E}_{\boldsymbol{n}, \lambda}$ & $\begin{array}{c}\text { see } \\
\text { table E4 }\end{array}$ & $\mathrm{nm}^{-1}$ & $\begin{array}{l}\text { Relative light distribution in the } \\
\text { spectrum of } 400-700 \mathrm{~nm}\end{array}$ & & \\
\hline
\end{tabular}

${ }^{1}$ The value for $m_{X^{\prime}}$ was estimated based on $m_{s}$ and the degree of reduction $(\gamma)$ of microalgal biomass and sugar $\left(\mathrm{CH}_{2} \mathrm{O}\right): m_{X^{\prime}}=m_{s} \cdot \gamma_{\text {sugar }} / \gamma_{\text {biomass }}$ 
Competition between antenna size mutant and wild type microalgae in mass culture

Table E4 Model parameters in dependency on the wavelength $(\lambda)$. The wavelength distribution $\left(E_{n, \lambda}\right)$ is based on ASTM G173-03 Reference Spectra derived from SMARTS v. 2.9.2. The absorption cross section $\left(a_{X^{\prime}, \lambda, W T}\right)$ was determined from a mass culture of $C$. sorokiniana wild type cells and has been normalized to functional biomass with respect to the minimum sugar fraction in functional biomass. The maximum yield of sugar on photons $\left(Y_{S / p h, m, \lambda}\right)$ is based on measurements with Chlorella pyrenoidosa (Emerson et al. 1943), but the data was multiplied by 1.1 (de Mooij 2015) to be in agreement with (Tanada 1951, Björkman et al. 1987, Evans 1987, Malkin et al. 1996).

\begin{tabular}{|c|c|c|c|}
\hline$\lambda$ & $E_{n, P A R, \lambda}$ & $\mathbf{a}_{X^{\prime}, \lambda, W T}$ & $Y_{S / p h, m, \lambda}$ \\
\hline [nm] & {$\left[\mathrm{nm}^{-1}\right]$} & {$\left[\frac{\mathrm{m}^{2}}{\mathrm{~mol}_{x^{\prime} \cdot \mathrm{nm}}}\right]$} & {$\left[\frac{\mathrm{mol}_{\mathrm{s}}}{\mathrm{mol}_{\mathrm{ph}} \cdot \mathrm{nm}}\right]$} \\
\hline 400 & $1.88 \mathrm{E}-03$ & 9.89 & $8.91 \mathrm{E}-02$ \\
\hline 401 & $1.96 \mathrm{E}-03$ & 10.01 & $8.91 \mathrm{E}-02$ \\
\hline 402 & $2.05 \mathrm{E}-03$ & \begin{tabular}{|l|}
10.14 \\
\end{tabular} & $8.91 \mathrm{E}-02$ \\
\hline 403 & $1.97 \mathrm{E}-03$ & 10.28 & $8.91 \mathrm{E}-02$ \\
\hline 404 & $2.01 \mathrm{E}-03$ & 10.44 & $8.91 \mathrm{E}-02$ \\
\hline 405 & $1.97 \mathrm{E}-03$ & 10.61 & $8.91 \mathrm{E}-02$ \\
\hline 406 & $1.92 \mathrm{E}-03$ & 10.78 & $8.91 \mathrm{E}-02$ \\
\hline 407 & $1.89 \mathrm{E}-03$ & 10.98 & $8.91 \mathrm{E}-02$ \\
\hline 408 & $1.98 \mathrm{E}-03$ & \begin{tabular}{|c|}
11.17 \\
\end{tabular} & $8.91 \mathrm{E}-02$ \\
\hline 409 & $2.12 \mathrm{E}-03$ & 11.35 & $8.91 \mathrm{E}-02$ \\
\hline 410 & $1.81 \mathrm{E}-03$ & 11.52 & $8.91 \mathrm{E}-02$ \\
\hline 411 & $2.04 \mathrm{E}-03$ & 11.69 & $8.91 \mathrm{E}-02$ \\
\hline 412 & 2.17E-03 & 11.85 & $8.91 \mathrm{E}-02$ \\
\hline 413 & $2.09 \mathrm{E}-03$ & 11.99 & $8.91 \mathrm{E}-02$ \\
\hline 414 & 2.07E-03 & 12.12 & $8.91 \mathrm{E}-02$ \\
\hline 415 & $2.15 \mathrm{E}-03$ & 12.23 & $8.80 \mathrm{E}-02$ \\
\hline 416 & $2.22 \mathrm{E}-03$ & 12.34 & $8.80 \mathrm{E}-02$ \\
\hline 417 & $2.17 \mathrm{E}-03$ & 12.43 & $8.80 \mathrm{E}-02$ \\
\hline 418 & $2.08 \mathrm{E}-03$ & 12.52 & $8.80 \mathrm{E}-02$ \\
\hline 419 & 2.17E-03 & 12.59 & $8.80 \mathrm{E}-02$ \\
\hline 420 & $1.99 \mathrm{E}-03$ & 12.66 & $8.80 \mathrm{E}-02$ \\
\hline 421 & $2.27 \mathrm{E}-03$ & 12.73 & $8.80 \mathrm{E}-02$ \\
\hline 422 & $2.24 \mathrm{E}-03$ & 12.80 & $8.80 \mathrm{E}-02$ \\
\hline 423 & $2.17 \mathrm{E}-03$ & 12.87 & $8.80 \mathrm{E}-02$ \\
\hline 424 & $2.17 \mathrm{E}-03$ & 12.95 & $8.69 \mathrm{E}-02$ \\
\hline 425 & $2.24 \mathrm{E}-03$ & 13.05 & $8.69 \mathrm{E}-02$ \\
\hline 426 & $2.18 \mathrm{E}-03$ & 13.16 & $8.69 \mathrm{E}-02$ \\
\hline 427 & $2.11 \mathrm{E}-03$ & 13.28 & $8.69 \mathrm{E}-02$ \\
\hline 428 & $2.14 \mathrm{E}-03$ & 13.40 & $8.69 \mathrm{E}-02$ \\
\hline 429 & $1.98 \mathrm{E}-03$ & 13.55 & $8.69 \mathrm{E}-02$ \\
\hline 430 & $1.59 \mathrm{E}-03$ & 13.68 & $8.69 \mathrm{E}-02$ \\
\hline 431 & $1.44 \mathrm{E}-03$ & 13.81 & $8.69 \mathrm{E}-02$ \\
\hline 432 & $2.41 \mathrm{E}-03$ & 13.93 & $8.69 \mathrm{E}-02$ \\
\hline 433 & $2.24 \mathrm{E}-03$ & 14.04 & $8.58 \mathrm{E}-02$ \\
\hline 434 & $2.08 \mathrm{E}-03$ & 14.13 & $8.58 \mathrm{E}-02$ \\
\hline 435 & $2.28 \mathrm{E}-03$ & 14.20 & $8.58 \mathrm{E}-02$ \\
\hline 436 & $2.51 \mathrm{E}-03$ & 14.23 & $8.58 \mathrm{E}-02$ \\
\hline 437 & $2.57 \mathrm{E}-03$ & 14.22 & $8.58 \mathrm{E}-02$ \\
\hline 438 & $2.26 \mathrm{E}-03$ & 14.18 & $8.58 \mathrm{E}-02$ \\
\hline 439 & $2.18 \mathrm{E}-03$ & 14.10 & $8.47 \mathrm{E}-02$ \\
\hline 440 & $2.51 \mathrm{E}-03$ & 14.01 & $8.47 \mathrm{E}-02$ \\
\hline 441 & $2.48 \mathrm{E}-03$ & 13.90 & $8.47 \mathrm{E}-02$ \\
\hline 442 & $2.66 \mathrm{E}-03$ & 13.75 & $8.47 \mathrm{E}-02$ \\
\hline 443 & $2.70 \mathrm{E}-03$ & 13.56 & $8.47 \mathrm{E}-02$ \\
\hline 444 & $2.64 \mathrm{E}-03$ & 13.33 & $8.47 \mathrm{E}-02$ \\
\hline 445 & $2.74 \mathrm{E}-03$ & 13.08 & $8.36 \mathrm{E}-02$ \\
\hline 446 & $2.47 \mathrm{E}-03$ & 12.80 & $8.36 \mathrm{E}-02$ \\
\hline 447 & $2.81 \mathrm{E}-03$ & 12.51 & $8.36 \mathrm{E}-02$ \\
\hline 448 & $2.85 \mathrm{E}-03$ & 12.22 & $8.25 \mathrm{E}-02$ \\
\hline 449 & $2.85 \mathrm{E}-03$ & 11.94 & $8.25 \mathrm{E}-02$ \\
\hline 450 & $2.96 \mathrm{E}-03$ & 11.67 & $8.25 \mathrm{E}-02$ \\
\hline
\end{tabular}

\begin{tabular}{|c|c|c|c|}
\hline$\lambda$ & $E_{n, P A R, \lambda}$ & $\mathbf{a}_{\mathbf{X}^{\prime}, \lambda, W T}$ & $Y_{S / p h, m, \lambda}$ \\
\hline$[\mathrm{nm}]$ & {$\left[\mathrm{nm}^{-1}\right]$} & {$\left[\frac{\mathrm{m}^{2}}{\mathrm{~mol}_{x^{\prime}} \cdot \mathrm{nm}}\right]$} & {$\left[\frac{\mathrm{mol}_{\mathrm{s}}}{\mathrm{mol}_{\mathrm{ph}} \cdot \mathrm{nm}}\right]$} \\
\hline 451 & $3.08 \mathrm{E}-03$ & 11.41 & $8.25 \mathrm{E}-02$ \\
\hline 452 & $2.95 \mathrm{E}-03$ & 11.19 & $8.25 \mathrm{E}-02$ \\
\hline 453 & $2.73 \mathrm{E}-03$ & 11.00 & $8.25 \mathrm{E}-02$ \\
\hline 454 & $2.94 \mathrm{E}-03$ & 10.85 & $8.14 \mathrm{E}-02$ \\
\hline 455 & $2.92 \mathrm{E}-03$ & 10.72 & $8.14 \mathrm{E}-02$ \\
\hline 456 & $3.02 \mathrm{E}-03$ & 10.62 & $8.14 \mathrm{E}-02$ \\
\hline 457 & $3.06 \mathrm{E}-03$ & 10.54 & $8.03 \mathrm{E}-02$ \\
\hline 458 & $3.00 \mathrm{E}-03$ & 10.49 & $8.03 \mathrm{E}-02$ \\
\hline 459 & $2.98 \mathrm{E}-03$ & 10.45 & $8.03 \mathrm{E}-02$ \\
\hline 460 & $2.97 \mathrm{E}-03$ & 10.42 & $7.92 \mathrm{E}-02$ \\
\hline 461 & $3.08 \mathrm{E}-03$ & 10.41 & $7.92 \mathrm{E}-02$ \\
\hline 462 & $3.11 \mathrm{E}-03$ & 10.39 & $7.92 \mathrm{E}-02$ \\
\hline 463 & 3.13E-03 & 10.39 & $7.92 \mathrm{E}-02$ \\
\hline 464 & 3.04E-03 & 10.39 & $7.92 \mathrm{E}-02$ \\
\hline 465 & $3.01 \mathrm{E}-03$ & 10.39 & $7.92 \mathrm{E}-02$ \\
\hline 466 & $3.08 \mathrm{E}-03$ & 10.40 & $7.81 \mathrm{E}-02$ \\
\hline 467 & $2.95 \mathrm{E}-03$ & 10.40 & $7.81 \mathrm{E}-02$ \\
\hline 468 & $3.08 \mathrm{E}-03$ & 10.40 & $7.81 \mathrm{E}-02$ \\
\hline 469 & $3.10 \mathrm{E}-03$ & 10.40 & $7.70 \mathrm{E}-02$ \\
\hline 470 & $2.99 \mathrm{E}-03$ & 10.40 & $7.70 \mathrm{E}-02$ \\
\hline 471 & $3.05 \mathrm{E}-03$ & 10.39 & $7.70 \mathrm{E}-02$ \\
\hline 472 & $3.21 \mathrm{E}-03$ & 10.38 & $7.59 \mathrm{E}-02$ \\
\hline 473 & $3.09 \mathrm{E}-03$ & 10.37 & $7.59 \mathrm{E}-02$ \\
\hline 474 & $3.13 \mathrm{E}-03$ & 10.34 & $7.59 \mathrm{E}-02$ \\
\hline 475 & $3.24 \mathrm{E}-03$ & 10.31 & $7.48 \mathrm{E}-02$ \\
\hline 476 & $3.14 \mathrm{E}-03$ & 10.28 & $7.48 \mathrm{E}-02$ \\
\hline 477 & $3.16 \mathrm{E}-03$ & 10.25 & $7.48 \mathrm{E}-02$ \\
\hline 478 & $3.27 \mathrm{E}-03$ & 10.22 & $7.37 \mathrm{E}-02$ \\
\hline 479 & $3.22 \mathrm{E}-03$ & 10.20 & $7.37 \mathrm{E}-02$ \\
\hline 480 & $3.28 \mathrm{E}-03$ & 10.18 & 7.37E-02 \\
\hline 481 & $3.28 \mathrm{E}-03$ & 10.16 & $7.37 \mathrm{E}-02$ \\
\hline 482 & $3.30 \mathrm{E}-03$ & 10.14 & $7.37 \mathrm{E}-02$ \\
\hline 483 & $3.27 \mathrm{E}-03$ & 10.12 & $7.37 \mathrm{E}-02$ \\
\hline 484 & $3.21 \mathrm{E}-03$ & 10.08 & $7.26 \mathrm{E}-02$ \\
\hline 485 & $3.21 \mathrm{E}-03$ & 10.03 & $7.26 \mathrm{E}-02$ \\
\hline 486 & $2.61 \mathrm{E}-03$ & 9.97 & $7.26 \mathrm{E}-02$ \\
\hline 487 & $2.93 \mathrm{E}-03$ & 9.89 & $7.26 \mathrm{E}-02$ \\
\hline 488 & $3.17 \mathrm{E}-03$ & 9.79 & $7.26 \mathrm{E}-02$ \\
\hline 489 & $3.00 \mathrm{E}-03$ & 9.66 & $7.26 \mathrm{E}-02$ \\
\hline 490 & $3.35 \mathrm{E}-03$ & 9.52 & $7.26 \mathrm{E}-02$ \\
\hline 491 & $3.23 \mathrm{E}-03$ & 9.35 & $7.26 \mathrm{E}-02$ \\
\hline 492 & $3.09 \mathrm{E}-03$ & 9.16 & $7.26 \mathrm{E}-02$ \\
\hline 493 & $3.31 \mathrm{E}-03$ & 8.97 & $7.37 \mathrm{E}-02$ \\
\hline 494 & $3.24 \mathrm{E}-03$ & 8.75 & $7.37 \mathrm{E}-02$ \\
\hline 495 & $3.44 \mathrm{E}-03$ & 8.52 & $7.37 \mathrm{E}-02$ \\
\hline 496 & $3.28 \mathrm{E}-03$ & 8.28 & $7.48 \mathrm{E}-02$ \\
\hline 497 & $3.34 \mathrm{E}-03$ & 8.02 & $7.48 \mathrm{E}-02$ \\
\hline 498 & $3.26 \mathrm{E}-03$ & 7.75 & $7.48 \mathrm{E}-02$ \\
\hline 499 & $3.26 \mathrm{E}-03$ & 7.47 & $7.70 \mathrm{E}-02$ \\
\hline 500 & $3.26 \mathrm{E}-03$ & 7.18 & $7.70 \mathrm{E}-02$ \\
\hline 501 & $3.17 \mathrm{E}-03$ & 6.89 & $7.70 \mathrm{E}-02$ \\
\hline
\end{tabular}

\begin{tabular}{|c|c|c|c|}
\hline$\lambda$ & $E_{n, P A R, \lambda}$ & $\mathbf{a}_{\mathbf{X}^{\prime}, \lambda, W T}$ & $Y_{S / p h, m, \lambda}$ \\
\hline$[\mathrm{nm}]$ & {$\left[\mathrm{nm}^{-1}\right]$} & {$\left[\frac{\mathrm{m}^{2}}{\mathrm{~mol}_{x^{\prime}} \cdot \mathrm{nm}}\right]$} & {$\left[\frac{\mathrm{mol}_{\mathrm{s}}}{\mathrm{mol}_{\mathrm{ph}} \cdot \mathrm{nm}}\right.$} \\
\hline 502 & $3.17 \mathrm{E}-03$ & 6.60 & $7.81 \mathrm{E}-02$ \\
\hline 503 & $3.32 \mathrm{E}-03$ & 6.31 & $7.81 \mathrm{E}-02$ \\
\hline 504 & $3.10 \mathrm{E}-03$ & 6.02 & $7.81 \mathrm{E}-02$ \\
\hline 505 & $3.33 \mathrm{E}-03$ & 5.74 & $7.92 \mathrm{E}-02$ \\
\hline 506 & $3.47 \mathrm{E}-03$ & 5.46 & $7.92 \mathrm{E}-02$ \\
\hline 507 & $3.33 \mathrm{E}-03$ & 5.18 & $7.92 \mathrm{E}-02$ \\
\hline 508 & $3.25 \mathrm{E}-03$ & 4.91 & $8.03 \mathrm{E}-02$ \\
\hline 509 & $3.41 \mathrm{E}-03$ & 4.65 & $8.03 \mathrm{E}-02$ \\
\hline 510 & $3.33 \mathrm{E}-03$ & 4.39 & $8.03 \mathrm{E}-02$ \\
\hline 511 & $3.40 \mathrm{E}-03$ & 4.16 & $8.14 \mathrm{E}-02$ \\
\hline 512 & $3.50 \mathrm{E}-03$ & 3.93 & $8.14 \mathrm{E}-02$ \\
\hline 513 & $3.29 \mathrm{E}-03$ & 3.71 & $8.14 \mathrm{E}-02$ \\
\hline 514 & $3.23 \mathrm{E}-03$ & 3.50 & $8.25 \mathrm{E}-02$ \\
\hline 515 & $3.33 \mathrm{E}-03$ & 3.30 & $8.25 \mathrm{E}-02$ \\
\hline 516 & $3.36 \mathrm{E}-03$ & 3.11 & $8.25 \mathrm{E}-02$ \\
\hline 517 & $2.75 \mathrm{E}-03$ & 2.94 & $8.36 \mathrm{E}-02$ \\
\hline 518 & $3.15 \mathrm{E}-03$ & 2.78 & $8.36 \mathrm{E}-02$ \\
\hline 519 & $3.06 \mathrm{E}-03$ & 2.63 & $8.36 \mathrm{E}-02$ \\
\hline 520 & $3.34 \mathrm{E}-03$ & 2.50 & $8.47 \mathrm{E}-02$ \\
\hline 521 & $3.37 \mathrm{E}-03$ & 2.38 & $8.47 \mathrm{E}-02$ \\
\hline 522 & $3.45 \mathrm{E}-03$ & 2.27 & $8.47 \mathrm{E}-02$ \\
\hline 523 & $3.26 \mathrm{E}-03$ & 2.17 & $8.58 \mathrm{E}-02$ \\
\hline 524 & $3.52 \mathrm{E}-03$ & 2.08 & $8.58 \mathrm{E}-02$ \\
\hline 525 & $3.49 \mathrm{E}-03$ & 2.00 & $8.58 \mathrm{E}-02$ \\
\hline 526 & $3.40 \mathrm{E}-03$ & 1.93 & $8.58 \mathrm{E}-02$ \\
\hline 527 & $2.98 \mathrm{E}-03$ & 1.87 & $8.58 \mathrm{E}-02$ \\
\hline 528 & $3.42 \mathrm{E}-03$ & 1.81 & $8.58 \mathrm{E}-02$ \\
\hline 529 & $3.59 \mathrm{E}-03$ & 1.76 & $8.69 \mathrm{E}-02$ \\
\hline 530 & $3.45 \mathrm{E}-03$ & 1.72 & $8.69 \mathrm{E}-02$ \\
\hline 531 & $3.65 \mathrm{E}-03$ & 1.68 & $8.69 \mathrm{E}-02$ \\
\hline 532 & $3.59 \mathrm{E}-03$ & 1.65 & $8.80 \mathrm{E}-02$ \\
\hline 533 & $3.21 \mathrm{E}-03$ & 1.62 & $8.80 \mathrm{E}-02$ \\
\hline 534 & $3.45 \mathrm{E}-03$ & 1.60 & $8.80 \mathrm{E}-02$ \\
\hline 535 & $3.51 \mathrm{E}-03$ & 1.58 & $8.91 \mathrm{E}-02$ \\
\hline 536 & $3.66 \mathrm{E}-03$ & 1.56 & $8.91 \mathrm{E}-02$ \\
\hline 537 & $3.40 \mathrm{E}-03$ & 1.54 & $8.91 \mathrm{E}-02$ \\
\hline 538 & $3.57 \mathrm{E}-03$ & 1.53 & $8.91 \mathrm{E}-02$ \\
\hline 539 & $3.49 \mathrm{E}-03$ & 1.52 & $8.91 \mathrm{E}-02$ \\
\hline 540 & $3.38 \mathrm{E}-03$ & 1.51 & $8.91 \mathrm{E}-02$ \\
\hline 541 & $3.25 \mathrm{E}-03$ & 1.50 & $9.02 \mathrm{E}-02$ \\
\hline 542 & $3.55 \mathrm{E}-03$ & 1.49 & $9.02 \mathrm{E}-02$ \\
\hline 543 & $3.49 \mathrm{E}-03$ & 1.48 & $9.02 \mathrm{E}-02$ \\
\hline 544 & $3.62 \mathrm{E}-03$ & 1.48 & $9.13 \mathrm{E}-02$ \\
\hline 545 & $3.55 \mathrm{E}-03$ & 1.47 & $9.13 \mathrm{E}-02$ \\
\hline 546 & $3.52 \mathrm{E}-03$ & 1.46 & $9.13 \mathrm{E}-02$ \\
\hline 547 & $3.57 \mathrm{E}-03$ & 1.45 & $9.24 \mathrm{E}-02$ \\
\hline 548 & $3.48 \mathrm{E}-03$ & 1.45 & $9.24 \mathrm{E}-02$ \\
\hline 549 & $3.59 \mathrm{E}-03$ & 1.44 & $9.24 \mathrm{E}-02$ \\
\hline 550 & $3.57 \mathrm{E}-03$ & 1.44 & $9.24 \mathrm{E}-02$ \\
\hline 551 & $3.58 \mathrm{E}-03$ & 1.43 & $9.24 \mathrm{E}-02$ \\
\hline 552 & $3.66 \mathrm{E}-03$ & 1.43 & $9.24 \mathrm{E}-02$ \\
\hline
\end{tabular}


Chapter 5

\begin{tabular}{|c|c|c|c|}
\hline$\lambda$ & $E_{n, P A R, \lambda}$ & $\mathbf{a}_{\mathbf{X}^{\prime}, \lambda, W T}$ & $Y_{S / p h, m, \lambda}$ \\
\hline$[\mathrm{nm}]$ & {$\left[\mathrm{nm}^{-1}\right]$} & {$\left[\frac{\mathrm{m}^{2}}{\mathrm{~mol}_{\mathrm{X}^{\prime}} \cdot \mathrm{nm}}\right]$} & {$\left[\frac{\mathrm{mol}_{\mathrm{s}}}{\mathrm{mol}_{\mathrm{ph}} \cdot \mathrm{nm}}\right.$} \\
\hline 553 & $3.56 \mathrm{E}-03$ & 1.43 & $9.35 \mathrm{E}-02$ \\
\hline 554 & $3.63 \mathrm{E}-03$ & 1.43 & $9.35 \mathrm{E}-02$ \\
\hline 555 & $3.66 \mathrm{E}-03$ & 1.43 & $9.35 \mathrm{E}-02$ \\
\hline 556 & $3.60 \mathrm{E}-03$ & 1.44 & $9.35 \mathrm{E}-02$ \\
\hline 557 & $3.52 \mathrm{E}-03$ & 1.44 & $9.35 \mathrm{E}-02$ \\
\hline 558 & $3.60 \mathrm{E}-03$ & 1.46 & $9.35 \mathrm{E}-02$ \\
\hline 559 & $3.42 \mathrm{E}-03$ & 1.47 & $9.46 \mathrm{E}-02$ \\
\hline 560 & $3.48 \mathrm{E}-03$ & 1.48 & $9.46 \mathrm{E}-02$ \\
\hline 561 & $3.69 \mathrm{E}-03$ & 1.50 & $9.46 \mathrm{E}-02$ \\
\hline 562 & $3.52 \mathrm{E}-03$ & 1.52 & $9.57 \mathrm{E}-02$ \\
\hline 563 & $3.66 \mathrm{E}-03$ & 1.54 & $9.57 \mathrm{E}-02$ \\
\hline 564 & $3.59 \mathrm{E}-03$ & 1.57 & $9.57 \mathrm{E}-02$ \\
\hline 565 & $3.62 \mathrm{E}-03$ & 1.61 & $9.57 \mathrm{E}-02$ \\
\hline 566 & $3.43 \mathrm{E}-03$ & 1.64 & $9.57 \mathrm{E}-02$ \\
\hline 567 & $3.66 \mathrm{E}-03$ & 1.68 & $9.57 \mathrm{E}-02$ \\
\hline 568 & $3.64 \mathrm{E}-03$ & 1.72 & $9.68 \mathrm{E}-02$ \\
\hline 569 & $3.55 \mathrm{E}-03$ & 1.76 & $9.68 \mathrm{E}-02$ \\
\hline 570 & $3.56 \mathrm{E}-03$ & 1.80 & $9.68 \mathrm{E}-02$ \\
\hline 571 & $3.45 \mathrm{E}-03$ & 1.85 & $9.79 \mathrm{E}-02$ \\
\hline 572 & $3.65 \mathrm{E}-03$ & 1.90 & $9.79 \mathrm{E}-02$ \\
\hline 573 & 3.67E-03 & 1.94 & $9.79 \mathrm{E}-02$ \\
\hline 574 & $3.66 \mathrm{E}-03$ & 1.99 & $9.79 \mathrm{E}-02$ \\
\hline 575 & $3.58 \mathrm{E}-03$ & 2.03 & $9.79 \mathrm{E}-02$ \\
\hline 576 & $3.56 \mathrm{E}-03$ & 2.06 & $9.79 \mathrm{E}-02$ \\
\hline 577 & $3.66 \mathrm{E}-03$ & 2.10 & $9.79 \mathrm{E}-02$ \\
\hline 578 & $3.55 \mathrm{E}-03$ & 2.14 & $9.79 \mathrm{E}-02$ \\
\hline 579 & $3.61 \mathrm{E}-03$ & 2.17 & $9.79 \mathrm{E}-02$ \\
\hline 580 & $3.67 \mathrm{E}-03$ & 2.21 & $9.90 \mathrm{E}-02$ \\
\hline 581 & $3.70 \mathrm{E}-03$ & 2.24 & $9.90 \mathrm{E}-02$ \\
\hline 582 & $3.76 \mathrm{E}-03$ & 2.27 & $9.90 \mathrm{E}-02$ \\
\hline 583 & $3.81 \mathrm{E}-03$ & 2.30 & $9.90 \mathrm{E}-02$ \\
\hline 584 & $3.81 \mathrm{E}-03$ & 2.33 & $9.90 \mathrm{E}-02$ \\
\hline 585 & $3.78 \mathrm{E}-03$ & 2.36 & $9.90 \mathrm{E}-02$ \\
\hline 586 & $3.70 \mathrm{E}-03$ & 2.39 & $9.90 \mathrm{E}-02$ \\
\hline 587 & $3.78 \mathrm{E}-03$ & 2.42 & $9.90 \mathrm{E}-02$ \\
\hline 588 & $3.70 \mathrm{E}-03$ & 2.46 & $9.90 \mathrm{E}-02$ \\
\hline 589 & $3.20 \mathrm{E}-03$ & 2.49 & $9.90 \mathrm{E}-02$ \\
\hline 590 & $3.41 \mathrm{E}-03$ & 2.51 & $9.90 \mathrm{E}-02$ \\
\hline 591 & $3.66 \mathrm{E}-03$ & 2.54 & $9.90 \mathrm{E}-02$ \\
\hline 592 & $3.58 \mathrm{E}-03$ & 2.56 & $9.90 \mathrm{E}-02$ \\
\hline 593 & $3.64 \mathrm{E}-03$ & 2.58 & $9.90 \mathrm{E}-02$ \\
\hline 594 & $3.63 \mathrm{E}-03$ & 2.60 & $9.90 \mathrm{E}-02$ \\
\hline 595 & $3.59 \mathrm{E}-03$ & 2.62 & $9.90 \mathrm{E}-02$ \\
\hline 596 & $3.71 \mathrm{E}-03$ & 2.64 & $9.90 \mathrm{E}-02$ \\
\hline 597 & $3.72 \mathrm{E}-03$ & 2.65 & $9.90 \mathrm{E}-02$ \\
\hline 598 & $3.68 \mathrm{E}-03$ & 2.67 & $9.90 \mathrm{E}-02$ \\
\hline 599 & $3.69 \mathrm{E}-03$ & 2.69 & $9.90 \mathrm{E}-02$ \\
\hline 600 & $3.73 \mathrm{E}-03$ & 2.71 & $9.90 \mathrm{E}-02$ \\
\hline 601 & $3.70 \mathrm{E}-03$ & 2.73 & $9.90 \mathrm{E}-02$ \\
\hline 602 & $3.65 \mathrm{E}-03$ & 2.76 & $9.90 \mathrm{E}-02$ \\
\hline 603 & $3.73 \mathrm{E}-03$ & 2.79 & $9.90 \mathrm{E}-02$ \\
\hline
\end{tabular}

\begin{tabular}{|c|c|c|c|}
\hline$\lambda$ & $E_{n, P A R, \lambda}$ & $\mathbf{a}_{\mathbf{X}^{\prime}, \lambda, W T}$ & $Y_{S / p h, m, \lambda}$ \\
\hline [nm] & {$\left[\mathrm{nm}^{-1}\right]$} & {$\left[\frac{\mathrm{m}^{2}}{\mathrm{~mol}_{\mathrm{X}^{\prime}} \cdot \mathrm{nm}}\right]$} & {$\left[\frac{\mathrm{mol}_{\mathrm{s}}}{\mathrm{mol}_{\mathrm{ph}} \cdot \mathrm{nm}}\right]$} \\
\hline 604 & $3.80 \mathrm{E}-03$ & 2.82 & $9.90 \mathrm{E}-02$ \\
\hline 605 & $3.80 \mathrm{E}-03$ & 2.85 & $9.90 \mathrm{E}-02$ \\
\hline 606 & $3.79 \mathrm{E}-03$ & 2.89 & $9.90 \mathrm{E}-02$ \\
\hline 607 & $3.82 \mathrm{E}-03$ & 2.93 & $9.90 \mathrm{E}-02$ \\
\hline 608 & $3.81 \mathrm{E}-03$ & 2.97 & $9.90 \mathrm{E}-02$ \\
\hline 609 & $3.79 \mathrm{E}-03$ & 3.01 & $9.90 \mathrm{E}-02$ \\
\hline 610 & $3.78 \mathrm{E}-03$ & 3.05 & $9.90 \mathrm{E}-02$ \\
\hline 611 & $3.77 \mathrm{E}-03$ & 3.09 & $9.90 \mathrm{E}-02$ \\
\hline 612 & $3.83 \mathrm{E}-03$ & 3.13 & $9.90 \mathrm{E}-02$ \\
\hline 613 & $3.78 \mathrm{E}-03$ & 3.18 & $9.90 \mathrm{E}-02$ \\
\hline 614 & $3.67 \mathrm{E}-03$ & 3.23 & $9.90 \mathrm{E}-02$ \\
\hline 615 & $3.81 \mathrm{E}-03$ & 3.27 & $9.90 \mathrm{E}-02$ \\
\hline 616 & $3.72 \mathrm{E}-03$ & 3.31 & $9.90 \mathrm{E}-02$ \\
\hline 617 & $3.68 \mathrm{E}-03$ & 3.36 & $9.90 \mathrm{E}-02$ \\
\hline 618 & $3.82 \mathrm{E}-03$ & 3.39 & $9.90 \mathrm{E}-02$ \\
\hline 619 & $3.85 \mathrm{E}-03$ & 3.43 & $9.90 \mathrm{E}-02$ \\
\hline 620 & $3.85 \mathrm{E}-03$ & 3.46 & $9.90 \mathrm{E}-02$ \\
\hline 621 & $3.88 \mathrm{E}-03$ & 3.48 & $9.90 \mathrm{E}-02$ \\
\hline 622 & $3.74 \mathrm{E}-03$ & 3.51 & $9.90 \mathrm{E}-02$ \\
\hline 623 & $3.72 \mathrm{E}-03$ & 3.52 & $9.90 \mathrm{E}-02$ \\
\hline 624 & $3.72 \mathrm{E}-03$ & 3.54 & $9.90 \mathrm{E}-02$ \\
\hline 625 & $3.70 \mathrm{E}-03$ & 3.55 & $9.90 \mathrm{E}-02$ \\
\hline 626 & $3.70 \mathrm{E}-03$ & 3.56 & $9.90 \mathrm{E}-02$ \\
\hline 627 & $3.81 \mathrm{E}-03$ & 3.57 & $9.90 \mathrm{E}-02$ \\
\hline 628 & $3.61 \mathrm{E}-03$ & 3.57 & $9.90 \mathrm{E}-02$ \\
\hline 629 & $3.75 \mathrm{E}-03$ & 3.58 & $9.90 \mathrm{E}-02$ \\
\hline 630 & $3.70 \mathrm{E}-03$ & 3.58 & $9.90 \mathrm{E}-02$ \\
\hline 631 & $3.77 \mathrm{E}-03$ & 3.57 & $9.90 \mathrm{E}-02$ \\
\hline 632 & $3.64 \mathrm{E}-03$ & 3.57 & $9.90 \mathrm{E}-02$ \\
\hline 633 & $3.87 \mathrm{E}-03$ & 3.57 & $9.90 \mathrm{E}-02$ \\
\hline 634 & $3.82 \mathrm{E}-03$ & 3.58 & $9.90 \mathrm{E}-02$ \\
\hline 635 & $3.87 \mathrm{E}-03$ & 3.60 & $9.90 \mathrm{E}-02$ \\
\hline 636 & $3.79 \mathrm{E}-03$ & 3.62 & $9.90 \mathrm{E}-02$ \\
\hline 637 & $3.93 \mathrm{E}-03$ & 3.67 & $9.79 \mathrm{E}-02$ \\
\hline 638 & $3.96 \mathrm{E}-03$ & 3.73 & $9.79 \mathrm{E}-02$ \\
\hline 639 & $3.95 \mathrm{E}-03$ & 3.82 & $9.79 \mathrm{E}-02$ \\
\hline 640 & $3.87 \mathrm{E}-03$ & 3.93 & $9.68 \mathrm{E}-02$ \\
\hline 641 & $3.88 \mathrm{E}-03$ & 4.06 & $9.68 \mathrm{E}-02$ \\
\hline 642 & $3.89 \mathrm{E}-03$ & 4.21 & $9.68 \mathrm{E}-02$ \\
\hline 643 & $3.94 \mathrm{E}-03$ & 4.39 & $9.57 \mathrm{E}-02$ \\
\hline 644 & $3.93 \mathrm{E}-03$ & 4.58 & $9.57 \mathrm{E}-02$ \\
\hline 645 & $3.96 \mathrm{E}-03$ & 4.78 & $9.57 \mathrm{E}-02$ \\
\hline 646 & $3.86 \mathrm{E}-03$ & 4.99 & $9.57 \mathrm{E}-02$ \\
\hline 647 & $3.84 \mathrm{E}-03$ & 5.21 & $9.57 \mathrm{E}-02$ \\
\hline 648 & $3.81 \mathrm{E}-03$ & 5.41 & 9.57E-02 \\
\hline 649 & $3.70 \mathrm{E}-03$ & 5.59 & $9.46 \mathrm{E}-02$ \\
\hline 650 & $3.73 \mathrm{E}-03$ & 5.74 & $9.46 \mathrm{E}-02$ \\
\hline 651 & $3.97 \mathrm{E}-03$ & 5.84 & $9.46 \mathrm{E}-02$ \\
\hline 652 & $3.82 \mathrm{E}-03$ & 5.90 & $9.46 \mathrm{E}-02$ \\
\hline 653 & $3.94 \mathrm{E}-03$ & 5.94 & $9.46 \mathrm{E}-02$ \\
\hline 654 & $3.90 \mathrm{E}-03$ & 5.97 & $9.46 \mathrm{E}-02$ \\
\hline
\end{tabular}

\begin{tabular}{|c|c|c|c|}
\hline$\lambda$ & $E_{n, P A R, \lambda}$ & $\mathbf{a}_{\mathbf{X}^{\prime}, \lambda, W T}$ & $Y_{S / p h, m, \lambda}$ \\
\hline$[\mathrm{nm}]$ & {$\left[\mathrm{nm}^{-1}\right]$} & {$\left[\frac{\mathrm{m}^{2}}{\mathrm{~mol} \mathrm{l}_{y \cdot \mathrm{nm}}}\right]$} & $\frac{\mathrm{mol}_{\mathrm{s}}}{\mathrm{mol}_{1} \cdot \mathrm{nm}}$ \\
\hline 655 & $3.73 \mathrm{E}-03$ & 6.01 & $9.46 \mathrm{E}-02$ \\
\hline 656 & $3.28 \mathrm{E}-03$ & 6.07 & $9.46 \mathrm{E}-02$ \\
\hline 657 & $3.43 \mathrm{E}-03$ & 6.17 & $9.46 \mathrm{E}-02$ \\
\hline 658 & $3.85 \mathrm{E}-03$ & 6.31 & $9.46 \mathrm{E}-02$ \\
\hline 659 & $3.87 \mathrm{E}-03$ & 6.51 & $9.46 \mathrm{E}-02$ \\
\hline 660 & $3.90 \mathrm{E}-03$ & 6.74 & $9.46 \mathrm{E}-02$ \\
\hline 661 & $3.89 \mathrm{E}-03$ & 7.01 & $9.46 \mathrm{E}-02$ \\
\hline 662 & $3.86 \mathrm{E}-03$ & 7.33 & $9.46 \mathrm{E}-02$ \\
\hline 663 & $3.87 \mathrm{E}-03$ & 7.67 & $9.46 \mathrm{E}-02$ \\
\hline 664 & $3.91 \mathrm{E}-03$ & 8.01 & $9.46 \mathrm{E}-02$ \\
\hline 665 & $3.99 \mathrm{E}-03$ & 8.36 & $9.46 \mathrm{E}-02$ \\
\hline 666 & $3.99 \mathrm{E}-03$ & 8.70 & $9.46 \mathrm{E}-02$ \\
\hline 667 & $3.97 \mathrm{E}-03$ & 9.01 & $9.57 \mathrm{E}-02$ \\
\hline 668 & $3.99 \mathrm{E}-03$ & 9.31 & $9.57 \mathrm{E}-02$ \\
\hline 669 & $4.06 \mathrm{E}-03$ & 9.58 & $9.57 \mathrm{E}-02$ \\
\hline 670 & $4.01 \mathrm{E}-03$ & 9.78 & $9.57 \mathrm{E}-02$ \\
\hline 671 & $4.01 \mathrm{E}-03$ & 9.95 & $9.57 \mathrm{E}-02$ \\
\hline 672 & $3.96 \mathrm{E}-03$ & 10.07 & $9.57 \mathrm{E}-02$ \\
\hline 673 & $4.00 \mathrm{E}-03$ & 10.15 & $9.68 \mathrm{E}-02$ \\
\hline 674 & $4.00 \mathrm{E}-03$ & 10.20 & $9.68 \mathrm{E}-02$ \\
\hline 675 & $3.97 \mathrm{E}-03$ & 10.22 & $9.68 \mathrm{E}-02$ \\
\hline 676 & $4.03 \mathrm{E}-03$ & 10.24 & $9.79 \mathrm{E}-02$ \\
\hline 677 & 4.00E-03 & 10.26 & $9.79 \mathrm{E}-02$ \\
\hline 678 & $4.02 \mathrm{E}-03$ & 10.27 & $9.79 \mathrm{E}-02$ \\
\hline 679 & $3.99 \mathrm{E}-03$ & 10.29 & $9.90 \mathrm{E}-02$ \\
\hline 680 & $4.01 \mathrm{E}-03$ & 10.26 & $9.90 \mathrm{E}-02$ \\
\hline 681 & $4.00 \mathrm{E}-03$ & 10.19 & $9.90 \mathrm{E}-02$ \\
\hline 682 & $4.02 \mathrm{E}-03$ & 10.02 & $9.90 \mathrm{E}-02$ \\
\hline 683 & $3.98 \mathrm{E}-03$ & 9.75 & $9.90 \mathrm{E}-02$ \\
\hline 684 & $3.96 \mathrm{E}-03$ & 9.37 & $9.90 \mathrm{E}-02$ \\
\hline 685 & $3.97 \mathrm{E}-03$ & 8.88 & $9.79 \mathrm{E}-02$ \\
\hline 686 & $3.89 \mathrm{E}-03$ & 8.29 & $9.79 \mathrm{E}-02$ \\
\hline 687 & $2.81 \mathrm{E}-03$ & 7.65 & $9.79 \mathrm{E}-02$ \\
\hline 688 & $3.25 \mathrm{E}-03$ & 6.96 & $9.02 \mathrm{E}-02$ \\
\hline 689 & $3.28 \mathrm{E}-03$ & 6.30 & $9.02 \mathrm{E}-02$ \\
\hline 690 & $3.44 \mathrm{E}-03$ & 5.66 & $9.02 \mathrm{E}-02$ \\
\hline 691 & $3.59 \mathrm{E}-03$ & 5.05 & $7.70 \mathrm{E}-02$ \\
\hline 692 & $3.70 \mathrm{E}-03$ & 4.51 & $7.70 \mathrm{E}-02$ \\
\hline 693 & $3.69 \mathrm{E}-03$ & 4.01 & $7.70 \mathrm{E}-02$ \\
\hline 694 & $3.65 \mathrm{E}-03$ & 3.56 & $6.71 \mathrm{E}-02$ \\
\hline 695 & $3.73 \mathrm{E}-03$ & 3.18 & $6.71 \mathrm{E}-02$ \\
\hline 696 & $3.72 \mathrm{E}-03$ & 2.84 & $6.71 \mathrm{E}-02$ \\
\hline 697 & $3.94 \mathrm{E}-03$ & 2.54 & $5.28 \mathrm{E}-02$ \\
\hline 698 & $3.88 \mathrm{E}-03$ & 2.27 & $5.28 \mathrm{E}-02$ \\
\hline 699 & $3.81 \mathrm{E}-03$ & 2.03 & $5.28 \mathrm{E}-02$ \\
\hline 700 & $3.79 \mathrm{E}-03$ & 1.82 & $4.51 \mathrm{E}-02$ \\
\hline & & & \\
\hline & & & \\
\hline & & & \\
\hline & & & \\
\hline & & & \\
\hline
\end{tabular}




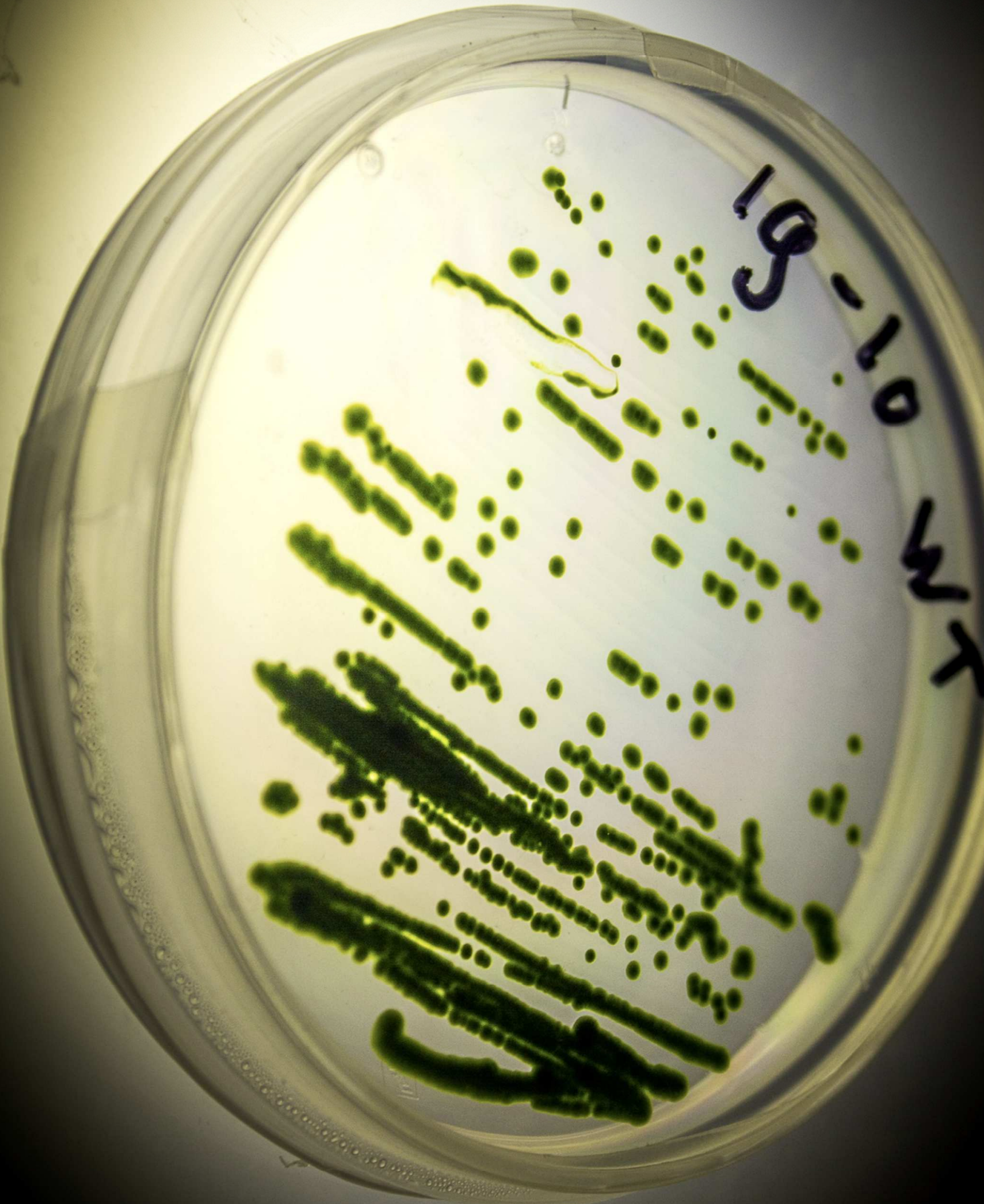




\section{Chapter 6:}

General discussion 


\subsection{Introduction}

There is an increasing industrial interest in microalgae as a production platform for biofuels, protein, lipids, pigments and carbohydrates (Hu et al. 2008, VanthoorKoopmans et al. 2013, Wijffels et al. 2013, Gangl et al. 2015). Similar to agricultural crops, microalgae have the ability to utilize sunlight as an energy source but they do not require arable land for their cultivation. The oil productivity of microalgae is considerably higher than that of conventional oil crops (Chisti 2007). Unlike crops, microalgae can be grown without investing biochemical energy on supportive structures such as roots and stems, which results in a higher product yield on light in algae than in agricultural crops. Harvesting of microalgae can be done continuously at any time of the year, instead of seasonally. Another advantage of microalgae is that their biomass concentration in a cultivation system can be controlled in order to maximize the solar light use efficiency and product yield. This makes microalgae promising hosts for the sustainable production of commodities.

Currently, the costs for cultivation and harvesting of microalgae are too high to produce bulk chemicals at a competitive price (Chisti 2007). Production costs can be lowered by increasing the productivity of cultivation systems. Because microalgae are a relatively new commercial production platform, there still is plenty of room for improvement from a technical as well as a biological perspective. In terms of biology, it can be argued that while agricultural crops have been domesticated for 10,000 years, which has resulted in higher productivities and improved resistance to pests (Doebley et al. 2006), the domestication of microalgae only just started. The possibility of genetic modification will speed up this process and create new opportunities.

Microalgae can be grown at various light intensities. At low light intensities, microalgae are capable of utilizing light energy at a very high efficiency. At increasing light intensities, growth is faster but the light use efficiency drops as the photosystems slowly become oversaturated and start to dissipate light energy as heat or fluorescence (Fig. 6.1). When microalgae are exposed to even higher light intensities, there is no additional growth and the excess of absorbed light energy is fully converted to heat due to activated photoprotective mechanisms (Li et al. 2009). Long exposure to very high light conditions can even damage the photosystems, which leads to photoinhibition. Already 
at $30 \%$ of maximum full sunlight most microalgae are being oversaturated, decreasing their light use efficiency. Photosystem oversaturation is the primary reason why photosynthetic efficiencies measured under outdoor conditions do not approximate that of the theoretical maximum (Ort et al. 2011). Preventing oversaturation is a serious challenge since light gradients in microalgal suspensions are steep starting at full sunlight intensity. In addition, microalgae have a high pigment content to be able to survive under low light conditions. Technical solutions to minimize light absorption per cell and provide a better light distribution in the photobioreactor are at hand, but this principle of light dilution (Tredici et al. 1998, Cuaresma et al. 2011, Dye et al. 2011, Zemke et al. 2013) often results in high photobioreactor material costs. Photosystem oversaturation is a biological problem and the most effective solution would therefore be to prevent that oversaturation occurs by means of genetic engineering (Chisti 2007).

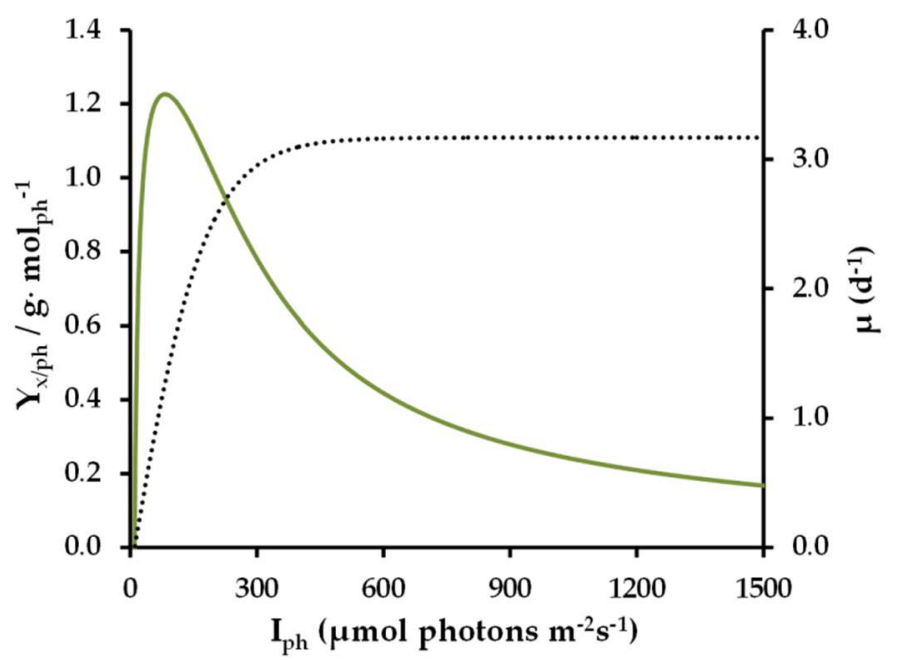

Fig. 6.1 The biomass specific growth rate ( $\mu$, dotted line) and the biomass yield on PAR photons ( $Y_{x / p h}$, solid line), i.e. a measure of the photosynthetic efficiency, are presented as a function of the light intensity $\left(I_{p h}\right)$ to which individual cells of Chlamydomonas reinhardtii are exposed. The data originates from the model presented in Chapter 2.

Obvious targets for genetic engineering would be the electron transport chain and the carbon fixation mechanisms in order to increase the light utilization rate and the specific growth rate. This is related to the fact that not light absorption, but the capacity to convert light to chemical energy is limiting under high light conditions. There has been a debate about the rate limiting step of light-saturated photosynthesis for many years, but no consensus has emerged (Sukenik et al. 1987, Zhu et al. 2007). It is not very likely that only a few biochemical conversions are limiting growth, and upregulation of the entire primary metabolism of photoautotrophic growth is too complex and not realistic in the near future. Therefore, an alternative is reducing the light uptake per cell by genetically reducing the size of the light harvesting antennae. Reducing the chlorophyll content to 
overcome the oversaturation bottleneck is a promising technique that may increase biomass productivity of a mass culture exposed to full sunlight (Mussgnug et al. 2007, Melis 2009, Ort et al. 2011, Formighieri et al. 2012, Perrine et al. 2012, Kwon et al. 2013, Oey et al. 2013). In this chapter, both the potential and the limitations of antenna size reduction are discussed. The requirements that are essential to create the ideal antenna size mutant with industrial potential are identified. It is also suggested how such a mutant can be obtained by applying an appropriate selection strategy.

\subsection{The creation of antenna size mutants}

A permanent antenna size reduction via genetic engineering can be obtained via two methods: site-directed mutagenesis or random mutagenesis combined with a screening and selection procedure. The advantage of site-directed mutagenesis is that specific changes can be made to the genes of interest without affecting other genes. A comprehensive understanding of the functioning of the photosystems is however lacking and also a complete genetic toolbox is not available for most of the green algae. Homologous recombination is very inefficient in microalgae (Gumpel et al. 1994). It is for this reason that most antenna size mutants created so far have been obtained by the well-established method of random mutagenesis. In practice however, random mutagenesis can only be used for simple mutations and screening of the generated mutant library can be very laborious. Besides, there is the risk of unintentionally affecting other gene functions, which may lead to reduced cell fitness. In chapter 2 four antenna size mutants were tested for their photosynthetic efficiency under mass culture conditions. None of the mutants demonstrated a higher photosynthetic efficiency than the wild type strain which most likely was due to a reduced fitness of the antenna size mutants. In theory random mutagenesis can be a suitable method to generate and characterize antenna size mutants, but so far no mutant has been generated that has an improved light use efficiency.

Depending on the strain, microalgae naturally alter their pigment content by two strategies: reduction of the total amount of reaction centers and / or reduction of the amount of chlorophyll per reaction center (Falkowski et al. 1991). The same strategies can be applied by means of genetic engineering either via site-directed mutagenesis or random mutagenesis. When the amount of reaction centers is reduced, less light is 
absorbed and higher biomass densities can be grown in photobioreactors. The advantage is that the light harvesting complexes have a normal composition and will therefore function properly (Formighieri 2015). The light use efficiency is however not necessarily increased as the excitation pressure per reaction center remains high and comparable to that of a wild type cell. Using the second strategy, the amount of light absorbed per reaction center is decreased. Genes of interest are for example all genes that are responsible for synthesis and assembly of the peripheral part of the light harvesting complexes (LHC), genes related to the synthesis of chlorophyll or LHCproteins that bind chlorophyll (Formighieri 2015). However, many of these binding proteins and the accompanying subunits have been shown to play a role in photoprotection mechanisms and energy distribution in the light harvesting complexes (Alboresi et al. 2009, Peers et al. 2009, Tokutsu et al. 2009, Tokutsu et al. 2013). For these reasons, truncating the light harvesting antenna might lead to impaired functioning of photosynthesis and photoprotection. Antenna size mutants have been created using both methods and as mentioned above both methods have their disadvantages (Formighieri 2015). Apparently the right method to obtain suitable mutants has not yet been found. As will be discussed later, a more targeted approach using improved screening and selection procedures could substantially increase the changes for obtaining suitable antenna size mutants.

\subsection{Decreasing oversaturation results in higher biomass productivities}

The main purpose of antenna size reduction is to limit light uptake rate per cell and with that the amount of photosystem oversaturation as mutants will require higher light intensities to become light saturated than wild type cells. It is already known that by applying a lower biomass specific light supply the amount of oversaturation is reduced and the photosynthetic efficiency increased (Barbosa et al. 2005, Zijffers et al. 2010, Sforza et al. 2015). In chapter 3 it was investigated, whether adjustment of the wavelength of the light could be exploited to maximize biomass productivity. This was only meant as a proof of principle and the method does not have a direct practical application to increase biomass productivity. As the microalgae pigments mostly absorb light in the blue and red region of the light spectrum (Fig 6.2), oversaturation can be minimized by spectrally optimizing the light source in order to illuminate the microalgae 
at wavelengths at which they have the lowest light harvesting capacity. The use of red and blue light would maximize oversaturation and decrease the obtained productivity compared to cultivation under sunlight, while by supplying the algae with green and yellow light, oversaturation would be minimized and the productivity will increase. To obtain the maximal possible productivity, it is important to adjust the biomass concentration for each light color to be able to absorb all the incident light. Using this approach, it was experimentally confirmed that the productivity of cultures exposed to red and blue light was considerably lower than cultures exposed to yellow and warm white light. Highest biomass productivity was obtained employing yellow light, which is not well absorbed and resulted in the least oversaturation.

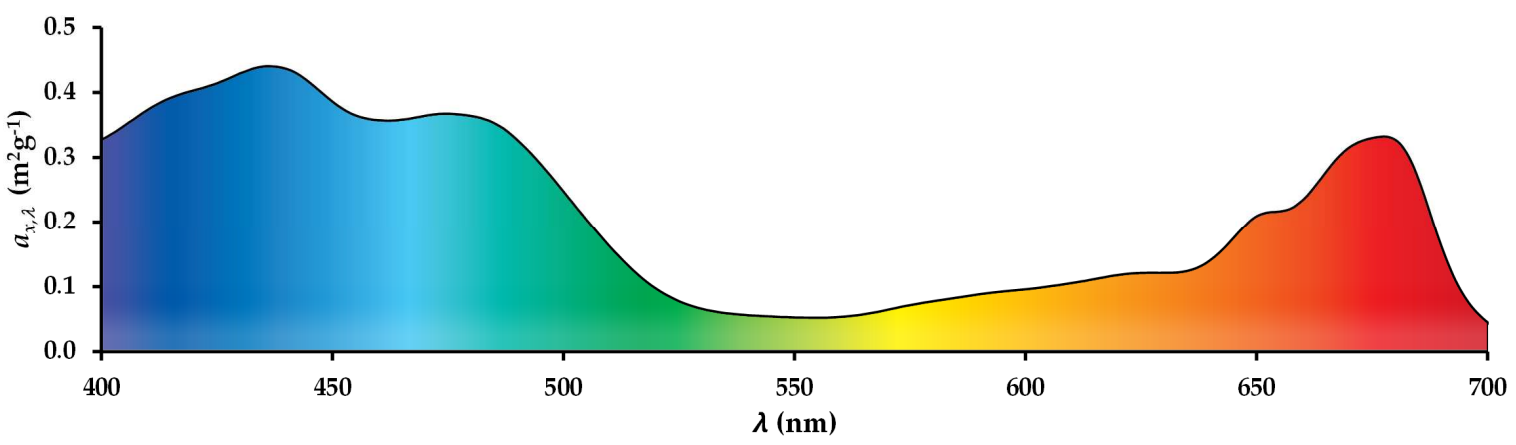

Fig. 6.2 Absorption cross section $\left(a_{x, \lambda}\right)$ of Chlamydomonas reinhardtii as a function of wavelength $(\lambda)$. It can be observed that there is a higher capacity for the absorption of blue and red light compared to green and yellow light. By exposing microalgae to yellow or green light, less light energy will be absorbed per cell, which reduces the effect of oversaturation under high light intensities.

Using the above mentioned approach, the amount of oversaturation in a simulated mass culture could be controlled by adjusting the wavelength of the artificial light source. In both approaches, the amount of light absorbed per photosystem can be reduced while employing high biomass densities. However, the use of artificial light for the purpose of microalgal bulk products is too expensive (Blanken et al. 2013) and therefore commercial application of the wavelength tuning technique is limited. Permanent antenna reduction is currently the only cost-effective solution to minimize photosystem oversaturation in microalgae mass cultures exposed to sunlight.

\subsection{Quantifying biomass productivity under mass culture conditions}

Since the idea of antenna size reduction was first considered as early as 1977 (Radmer et al. 1977), much effort was spent on estimating possible gains in productivity. 
Unequivocal experimental proof of increased productivity using antenna size mutants has not yet been obtained as the used experimental setups are inadequate, the results are misinterpreted, and the extrapolation of data to large scale conditions is often incorrect. In many cases wrong definitions are used for 'productivity' (Cazzaniga et al. 2014) and 'growth rate' (Perrine et al. 2012). This has led to the often quoted claim that antenna size mutant technology could triple the biomass productivity compared to wild type cells (Melis 2009), which is not-substantiated.

A PI (photosynthesis-irradiance) curve illustrates the photosynthetic activity of algae as a function of light intensity. A PI curve is usually based on the measurement of photosynthetic oxygen production by dilute microalgae cultures during short (minutescale) exposure to increasing light intensities. PI curves can be used to demonstrate that antenna size mutants are light saturated at higher light intensities than wild type cells (Nakajima et al. 2000, Kirst et al. 2012, Perrine et al. 2012). PI curves can also be used to show that antenna size mutants have a higher photosynthetic activity per unit of chlorophyll, as they are reduced in their chlorophyll content. However, PI curve that are expressed on a chlorophyll basis cannot be directly used to assess biomass productivity of antenna size mutants and wild type strains under mass culture conditions. The main reason for this is the fact that chlorophyll content is a poor proxy for light absorption. Due to the packing effect of chlorophylls in the antenna complex the effective absorption cross section of chlorophyll increases at reduced antenna size (Duyens 1956, Morel et al. 1981). This effect is not accounted for when normalizing photosynthesis rates to chlorophyll. In addition, light absorption by other pigments than chlorophylls is not taken into account. Moreover, the data obtained by a PI curve must be re-calculated into a hypothetical productivity of a microalgal mass culture based on an assumed light gradient. Clearly there is a need for a more accurate measurement of light absorption by antenna size mutants during PI measurements. A direct measurement of mass culture productivity at laboratory scale therefore should be preferred to assess the potential of antenna size mutants. The 'mass culture conditions' that are referred to in this thesis are defined as follows: A high incident light intensity that is representative of sunlight conditions around solar noon (e.g. $1500 \mu \mathrm{mol}$ photons $\mathrm{m}^{-2} \mathrm{~s}^{-1}$ for the Netherlands) and a biomass concentration that is high enough to absorb virtually all of the incident light energy (Fig 6.3). 

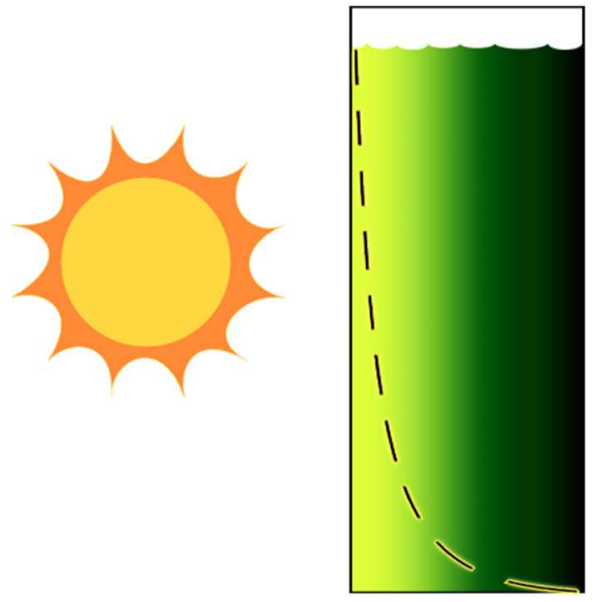

Fig. 6.3 Schematic drawing of microalgae mass culture conditions. The dashed line shows the light intensity gradient from full sunlight intensity to darkness at the back side of the culture. All the incident sunlight is absorbed by the culture as a result of a high biomass concentration. Three zones can be distinguished. Close to the reactor surface there is a very high light intensity, which results in a high level of oversaturation (yellow zone). In the middle of the culture, there is a narrow zone where there is no oversaturation and the photosynthetic efficiency is high (bright green zone). Close to the back side of the reactor, light intensity is near zero as all the incident light has been absorbed and microalgal growth is minimal (black zone).

In this thesis an experimental setup was presented (see Fig. 6.4) that allows for the measurement of biomass productivity under mass culture conditions. By employing turbidostat control, mass culture conditions could be simulated in continuously operated lab-scale photobioreactors containing high biomass densities. This resulted in a light gradient going from sunlight intensity to a value close to the compensation point of photosynthesis at the backside of the photobioreactor. Biomass production was determined by daily measurement of the diluted volume combined with the determination of the biomass dry weight concentration. By measuring the incident and outgoing light intensity (yellow arrows in Fig. 6.4), it was determined how much light was absorbed to produce the harvested biomass. This allowed calculation of the biomass yield on light, which is a direct measure of the photosynthetic efficiency. Using this experiment setup, also the contribution of cell maintenance is included, as well as other effects that are not easy to measure in minute-scale PI measurements, such as photoacclimation. In a mass culture the absorption cross section is adjusted to the light conditions and this influences the biomass productivity that is measured during one entire cultivation day. In addition, a controlled continuous reactor system allows better control of the $\mathrm{pH}$ and of $\mathrm{O}_{2}$ and $\mathrm{CO}_{2}$ concentrations and this ensures that the available light energy is the limiting factor for growth. For a detailed description of the used experimental setup and its operation, please refer to chapter 2 . The described experimental setup could be further improved by simulation day / night cycles and temperature fluctuations to better mimic outdoor conditions. 


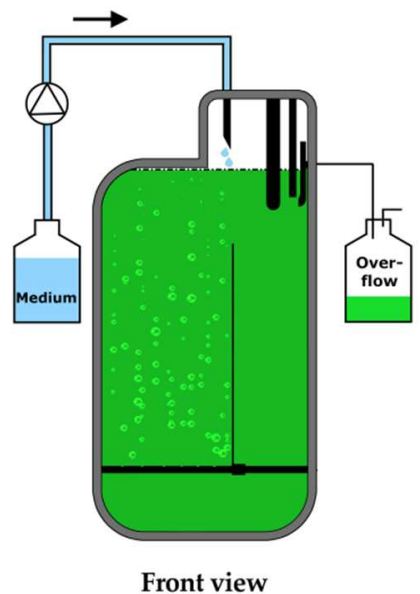

Front view

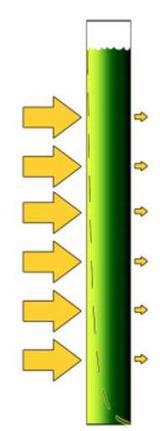

Side view
Fig. 6.4 Front and side view of a photobioreactor that is operated under simulated mass culture conditions. The large yellow arrows indicate the incident light and the small yellow arrows indicate the outgoing light intensity.

By both laboratory experiments or a mathematical model, an estimation can be made of the possible biomass productivity of antenna size mutants. As was described in chapter 2 , no experimental proof could be delivered that antenna reduction increases biomass productivity. The tested mutants all seemed to suffer from a reduced cell fitness. Since no suitable antenna size mutant was available for further research, a model approach was followed (chapter 5) to estimate the increase in productivity employing antenna size mutants under outdoor conditions. Important factors such as day/night cycles, spectral changes along the light path, and carbon partitioning were taken into account. A wide range of absorption cross section reductions was simulated for Chlorella sorokiniana mutants. It was concluded that a location of high irradiance is required to obtain a substantial increase in productivity using antenna reduction technology. The model simulations showed that under optimal conditions such as a location of high irradiance (Tunisia), the estimated productivity is about $40 \%$ higher than predicted for the wildtype. This is in marked contrast with the claim made before (Melis 2009) that biomass productivity could be tripled using antenna size reduction, but for our estimation many important factors were taken into consideration, which are discussed in chapter 5. As discussed before, oversaturation is difficult to overcome and an increase in productivity up to $40 \%$ is a major achievement under outdoor conditions.

\subsection{The role and application of the photoacclimation response}

In chapter 4 it was investigated whether high light acclimated cells could be a good indicator of antenna size mutant performance because of their reduced pigment content. It was concluded that high light acclimated cells do not adequately mimic ideal antenna 
size mutants. The high light acclimated cells were ascertained to have a lower pigmentation, but this did not increase the photosynthetic efficiency. The most likely explanation is that they are optimized to maximize light energy dissipation in order to prevent photodamage, mainly for the purpose of survival instead of enhanced biomass formation. It became clear that the natural process of photoacclimation cannot be exploited to improve biomass productivity in wild type mass cultures. This is because of the non-permanent character of the high light phenotype, the possible activation of dissipation mechanisms, the faster-than-expected pigment accumulation after a transfer to mass culture conditions, and possibly the energetic costs of the photoacclimation response itself. The photoacclimation response could however be beneficial for growth of microalgae in biofilms, where cells are not alternating between low and high light conditions, which allows acclimation to the local light intensity. Photoprotection and dissipation mechanisms would then only have to be active at the top of the biofilm, where cells will exhibit low pigmentation. In the deeper parts of the biofilm, with low light levels, microalgae will exhibit a high pigment content, while light dissipation mechanisms do not have to be active.

The idea that only a reduction of the chlorophyll content of microalgae is sufficient to maximize light use efficiency in mass cultures might be too simplistic. As was experimentally demonstrated in chapter 2, antenna size mutants are indeed more transparent and have a lower light absorption rate than wild type cells, but it remains unclear how the light intensity the cells are exposed to is detected and how this affects biological processes relevant for a balanced light distribution in the cells. Light intensity is for example not necessarily sensed via the absorbed amount of light per photosystem. Several types of photosensing proteins exist (Hellingwerf et al. 1996) that could, via biological signaling, activate mechanisms such as photoacclimation and photoprotection, as was discussed before in chapter 3 . It can be concluded that photoacclimation is a very complex process that is poorly understood. A better understanding of the mechanisms involved in photoacclimation could contribute to the generation of better antenna size mutants without a reduced cell fitness.

Researchers have succeeded in creating microalgae with a so-called locked in high light phenotype (Bailey et al. 2013), but as the experiments described in chapter 4 show this 
is probably not the way to maximize the photosynthetic efficiency of microalgae mass cultures. Instead, to create the ideal microalgal production strain, a comprehensive inventory should be made of genes associated to high light conditions that are essential for both survival as well as for efficient light utilization of antenna size mutants. On the one hand, some photoacclimation responses might be essential for proper functioning in a mass culture. On the other hand, genes related to excessive dissipation of light energy should, as much as possible, be avoided. In this way a better balance could be obtained between the light absorption rate and the downstream biochemical processes.

\subsection{Maintaining an antenna size mutant monoculture}

Antenna size mutants are reduced in their light harvesting capacity and as a result they will grow slower than full antenna organisms (i.e. wild type) under the same light conditions. Consequently, antenna size mutants will be easily overgrown which counteracts the potential gain in reactor productivity as was confirmed in the model estimations of chapter 5. With increasing contamination level and with increasing antenna size reduction, the mutants were overgrown in an earlier stage and this substantially decreased the gain in productivity that could be obtained using antenna size reduction over the entire simulated summer period of 100 days. It is essential to create antenna size mutants that do not compromise their competitiveness. Alternatively, cost-effective selective pressure techniques should be applied to steer competition in the direction of the mutants. This can be realized in several ways, two examples are explained below.

\section{High light treatment to damage wild type cells}

As antenna size mutants are in theory more tolerant to high light conditions than cells with a full antenna, this property might be used to their advantage. A treatment at very high light conditions could be realized by creating a bypass in the photobioreactor where the reactor is very thin and conditions are such that mutants are not, or hardly, photodamaged while wild type cells should be severely harmed. Alternatively part of the reactor is exposed to concentrated sunlight by using a lens. The goal is to reduce the biomass specific growth rate of wild type cells to values below that of the mutants, as this will eventually lead to washout of the wild type cells, assuming continuous 
cultivation is applied. Especially when using antenna size mutants with very low pigmentation, it might be feasible to exploit their difference in light tolerance compared to organisms with normal pigmentation.

\section{The addition of another trait to the antenna size mutant}

To compensate for the selective disadvantage of the antenna size mutant, the strain could be equipped with another property that increases its survival chances in the cultivation system. The mutant could for example be optimized to maintain high growth rates at lower temperatures while tolerating periods of very high temperature (deBashan et al. 2008). Tolerance to high salt concentrations (Jaeger 2015), high oxygen concentrations (Ghirardi et al. 1997, Raso et al. 2012, Sousa et al. 2013) or high shear forces (Michels et al. 2010) are other options. By specifically applying such a selection pressure, chances are smaller that invasive species will be able to outcompete the antenna size mutant.

\subsection{Obtaining better mutants via high light selective pressure or high throughput productivity screening}

Using the current antenna size mutant generation and selection methods, no suitable antenna size mutant has been obtained that has an improved photosynthetic efficiency. This could indicate that either the selection criteria are inadequate or that no appropriate selection pressure is being applied during the selection of the mutants. In this section, it is discussed how antenna size mutant generation might be improved by searching for low-pigmented microalgae in selective environments and by applying a more effective screening strategy using selective pressure on a generated mutant library. The discussed methods would deliver more robust light tolerant antenna size mutants that are expected to show higher maximal biomass productivity than the existing antenna size mutants.

\subsubsection{Obtaining low-pigmented microalgae from nature}

Traditionally in plant breeding, interesting strains or traits were selected from a large population and only the ones showing improved characteristics compared to the existing production strain were selected for further breeding. It would be interesting to 
look for microalgae strains with a reduced pigment content in nature in order to maximize light use efficiency. Such a strain would be a very good starting point for further optimization using mutagenesis in future research. It is however questionable whether a low-pigmented microalgal strain can be found in nature and this also raises the question whether a selective environment exists that rewards a small antenna and a high light use efficiency. Not all desired characteristics can be obtained via a selective environment. An environment where it is a competitive advantage to leave light energy to photosynthetic competitors, will be difficult to find or construct (Mooij et al. 2015). Maximization of individual cell survival is completely different from maximizing the light use efficiency of a mass culture (Anten 2005). In nature cells will only survive if they are more successful in reproduction than others. Absorption of excessive amounts of light, even though a large fraction will be wasted under high light conditions, may reduce the survival rate of competing photosynthetic organisms and a large antenna size therefore gives a selective advantage to the individual cell. However, the recent discovery of a new Chlorella strain that possesses a natural small antenna and an exotic energy dissipation mechanism is both surprising and promising (Treves et al. 2016) and could provide leads for the development of high light tolerant strains that efficiently convert light energy into biomass.

\subsubsection{Selective pressure using high light conditions}

A way to select for industrially relevant mutants in the generated mutant library is to create a selective environment (Mooij et al. 2015) that rewards a small antenna. The fact that recently a new Chlorella strain with a small absorption cross section has been isolated from an extreme desert environment (Treves et al. 2016), suggests that conditions exist under which a low pigment content can be advantageous. The mutant library could be constructed based on this new desert strain as its genotype is probably more similar to the target antenna size mutant than that of other wild type strains. The most obvious choice would be to choose for extremely high light conditions (3000 $\mu \mathrm{mol}$ photons $\mathrm{m}^{-2} \mathrm{~s}^{-1}$ ) that would severely photodamage and eliminate full antenna species while the ideal antenna size mutant should be able to survive these conditions. This experiment can be performed in a chemostat photobioreactor with a dilution rate equal to $80-90 \%$ of the known maximum specific growth rate. In this way mutants may be 
found that have a reduced light absorption capacity while maintaining the capacity to grow at their maximum growth rate, which is a good indicator of their fitness. The advantage of this approach is that the entire mutant library can be used to inoculate a reactor, which eliminates time consuming isolation steps. A possible outcome might be a mutant with superior non photochemical quenching capacity, i.e. mutants that are very good at wasting light energy. It is however arguable whether such high light intensities can be more effectively controlled by means of dissipation instead of reducing light absorption. Therefore, these mutants would be very promising candidates for lab scale productivity analysis using an experimental setup as described in Chapter 2 .

\subsubsection{High throughput screening for biomass productivity}

Conventional screening methods that are used after a mutant library has been created by random mutagenesis, are primarily based on chlorophyll content via measurement of chlorophyll fluorescence. In this way mutants are selected for further characterization, after which first simple growth tests and sometimes more advanced measurements under production conditions are performed. Unfortunately, the experimental conditions are often poorly defined and the results sometimes incorrectly interpreted. A good example of a study where, in addition to the chlorophyll content, also growth, NPQ and quantum yield were taken into account in an early stage is the work of Perin et al. (2015). Still, their initial screening is a time consuming process that consist of colony picking followed up by several weeks of growing the biomass before the actual screening takes place. Keeping in mind that not pigment reduction but productivity improvement is the aim of antenna size reduction, it would be more effective to include a productivity assessment, or a high light tolerance assessment, as early as possible in the selection process using high throughput screening technologies. Two methods are suggested:

A mutant library is generated via a random mutagenesis technique. The mutants are grown on agar plates under very high light conditions $\left(3000 \mu \mathrm{mol}\right.$ photons $\left.\mathrm{m}^{-2} \mathrm{~s}^{-1}\right)$ and the surviving mutants can be isolated and directly transferred to miniaturized cell analysis systems such as the lab-on-a-chip systems (Dufva 2009, Schaap et al. 2012) or small fluidic devices with chemostat and turbidostat options (Lee et al. 2011). These devices could be optimized to measure performance under high light conditions or even 
the biomass productivity under conditions that mimic mass culture conditions. High throughput microplate-based analysis of microalgal growth rate could also be valuable as an indication of biomass productivity (Van Wagenen et al. 2014).

Alternatively, when the mutant library is available in suspension form, it can be exposed to a very high irradiance. Subsequently, high throughput flow cytometric procedures (Mendoza et al. 2008, Manandhar-Shrestha et al. 2013) could be applied to select mutants with low chlorophyll fluorescence or other interesting fluorescent parameters (Maxwell et al. 2000). Subsequently, the selected cells can be analyzed via high throughput cell analysis systems to assess growth and fitness as mentioned above.

\subsection{Conclusions}

By reducing oversaturation of the photosynthetic machinery higher biomass productivities can be obtained in microalgae mass cultures. However, the evaluated antenna size mutants in this study did not exhibit the expected productivity improvement in a large amount of long-term photobioreactor experiments. Observations indicated a reduced fitness of the mutant cultures caused by a high light intensity. The antenna alterations are expected to have caused impaired photoprotection mechanisms or an imbalanced light distribution in the photosystems. The process of photoacclimation could not be exploited to increase the productivity of wild type microalgae in mass cultures, though for growth in biofilms local acclimation to the light conditions might prove advantageous. Based on the presented theoretical model, antenna size mutants could increase the biomass productivity under outdoor conditions in a high light environment by about $40 \%$. In mass cultures, antenna size mutants have a lower competitiveness than full antenna species and contamination with the latter substantially decreases the potential gain in productivity. The competitive strength of mutants needs to be improved to obtain antenna size mutants that are industrially relevant. In addition, an advanced genetic toolbox, a better understanding of photoprotection mechanisms and a multidisciplinary approach are of crucial importance to fully exploit the potential of antenna size reduction in the future. For the generation of future antenna size mutants, in addition to the chlorophyll content, more emphasis should be placed on high light tolerance and high productivity in an early stage. By creating a selective environment with extremely high light intensities, more robust light tolerant mutants may be obtained. 


\section{References}


Abiusi, F, G. Sampietro, G. Marturano, N. Biondi, L. Rodolfi, M. D'Ottavio and M. R. Tredici (2014). Growth, photosynthetic efficiency, and biochemical composition of Tetraselmis suecica F\&M-M33 grown with LEDs of different colors. Biotechnology and bioengineering 111(5): 956-964.

Agostoni, M., B. F. Lucker, M. A. Smith, A. Kanazawa, G. J. Blanchard, D. M. Kramer and B. L. Montgomery (2016). Competition-based phenotyping reveals a fitness cost for maintaining phycobilisomes under fluctuating light in the cyanobacterium Fremyella diplosiphon. Algal Research 15: 110-119.

Al-Najjar, M. A., D. De Beer, M. Kühl and L. Polerecky (2012). Light utilization efficiency in photosynthetic microbial mats. Environmental microbiology 14(4): 982-992.

Alboresi, A., M. Ballottari, R. Hienerwadel, G. M. Giacometti and T. Morosinotto (2009). Antenna complexes protect photosystem I from photoinhibition. BMC Plant Biol 9(1): 71.

Allen, J. F. (2003). Cyclic, pseudocyclic and noncyclic photophosphorylation: new links in the chain. Trends in plant science 8(1): 15-19.

Alnaser, W. E., B. Eliagoubi, A. Al-Kalak, H. Trabelsi, M. Al-Maalej, H. M. El-Sayed and M. Alloush (2004). First solar radiation atlas for the Arab world. Renewable Energy 29(7): 10851107.

Anten, N. P. (2005). Optimal photosynthetic characteristics of individual plants in vegetation stands and implications for species coexistence. Annals of Botany 95(3): 495-506.

Arnold, W. (1949). A calorimetric determination of the quantum yield in photosynthesis. Photosynthesis in Plants 2: 273.

Baba, M., F. Kikuta, I. Suzuki, M. M. Watanabe and Y. Shiraiwa (2012). Wavelength specificity of growth, photosynthesis, and hydrocarbon production in the oil-producing green alga Botryococcus braunii. Bioresource technology 109: 266-270.

Bailey, S., J. McCarren, S. L. Lieberman, J. E. Meuser, A. E. Romano, D. Yee, L. Soriaga, R. C. Brown, J. C. Weissman and R. C. Prince (2013). Algal mutants having a locked-in high light acclimated phenotype, Google Patents.

Barbosa, M. J., J. W. Zijffers, A. Nisworo, W. Vaes, J. van Schoonhoven and R. H. Wijffels (2005). Optimization of biomass, vitamins, and carotenoid yield on light energy in a flat-panel reactor using the A-stat technique. Biotechnology and bioengineering 89(2): 233-242.

Berner, F., K. Heimann and M. Sheehan (2014). Microalgal biofilms for biomass production. Journal of Applied Phycology: 1-12.

Björkman, O. and B. Demmig (1987). Photon yield of 02 evolution and chlorophyll fluorescence characteristics at $77 \mathrm{~K}$ among vascular plants of diverse origins. Planta 170(4): 489-504.

Blanken, W., M. Cuaresma, R. H. Wijffels and M. Janssen (2013). Cultivation of microalgae on artificial light comes at a cost. Algal Research 2(4): 333-340.

Blanken, W., M. Janssen, M. Cuaresma, Z. Libor, T. Bhaiji and R. Wijffels (2014). Biofilm growth of Chlorella sorokiniana in a rotating biological contactor based photobioreactor. Biotechnology and bioengineering 111(12): 2436-2445. 
Blanken, W., P. R. Postma, L. de Winter, R. H. Wijffels and M. Janssen (2016). Predicting microalgae growth. Algal Research 14: 28-38.

Bonente, G., C. Formighieri, M. Mantelli, C. Catalanotti, G. Giuliano, T. Morosinotto and R. Bassi (2011). Mutagenesis and phenotypic selection as a strategy toward domestication of Chlamydomonas reinhardtii strains for improved performance in photobioreactors. Photosynthesis Res 108(2-3): 107-120.

Bonente, G., S. Pippa, S. Castellano, R. Bassi and M. Ballottari (2012). Acclimation of Chlamydomonas reinhardtii to different growth irradiances. Journal of Biological Chemistry 287(8): 5833-5847.

Cazzaniga, S., L. Dall'Osto, J. Szaub, L. Scibilia, M. Ballottari, S. Purton and R. Bassi (2014). Domestication of the green alga Chlorella sorokiniana: reduction of antenna size improves light-use efficiency in a photobioreactor. Biotechnology for biofuels 7(1): 157.

Chaâbane, M., M. Masmoudi and K. Medhioub (2004). Determination of Linke turbidity factor from solar radiation measurement in northern Tunisia. Renewable Energy 29(13): 20652076.

Chisti, Y. (2007). Biodiesel from microalgae. Biotechnology Advances 25(3): 294-306.

Chow, W. S., A. Melis and J. M. Anderson (1990). Adjustments of photosystem stoichiometry in chloroplasts improve the quantum efficiency of photosynthesis. Proceedings of the National Academy of Sciences 87(19): 7502-7506.

Cooper, P. I. (1969). The absorption of radiation in solar stills. Solar Energy 12(3): 333-346.

Costa, B. S., A. Jungandreas, T. Jakob, W. Weisheit, M. Mittag and C. Wilhelm (2013). Blue light is essential for high light acclimation and photoprotection in the diatom Phaeodactylum tricornutum. Journal of Experimental Botany 64(2): 483-493.

Cuaresma, M., M. Janssen, E. J. Van den End, C. Vílchez and R. H. Wijffels (2011). Luminostat operation: A tool to maximize microalgae photosynthetic efficiency in photobioreactors during the daily light cycle? Bioresource technology 102(17): 7871-7878.

Cuaresma, M., M. Janssen, C. Vílchez and R. H. Wijffels (2011). Horizontal or vertical photobioreactors? How to improve microalgae photosynthetic efficiency. Bioresource technology 102(8): 5129-5137.

de-Bashan, L. E., A. Trejo, V. A. Huss, J.-P. Hernandez and Y. Bashan (2008). Chlorella sorokiniana UTEX 2805, a heat and intense, sunlight-tolerant microalga with potential for removing ammonium from wastewater. Bioresource technology 99(11): 4980-4989.

de Mooij, T., G. de Vries, C. Latsos, R. H. Wijffels and M. Janssen (2016). Impact of light color on photobioreactor productivity. Algal Research 15: 32-42.

de Mooij, T., M. Janssen, O. Cerezo-Chinarro, J. H. Mussgnug, O. Kruse, M. Ballottari, R. Bassi, S. Bujaldon, F.-A. Wollman and R. H. Wijffels (2014). Antenna size reduction as a strategy to increase biomass productivity: a great potential not yet realized. Journal of Applied Phycology 27(3): 1063-1077.

De Vitry, C. and F.-A. Wollman (1988). Changes in phosphorylation of thylakoid membrane proteins in light-harvesting complex mutants from Chlamydomonas reinhardtii. BBABioenergetics 933(3): 444-449. 
Deblois, C. P., A. Marchand and P. Juneau (2013). Comparison of Photoacclimation in Twelve Freshwater Photoautotrophs (Chlorophyte, Bacillaryophyte, Cryptophyte and Cyanophyte) Isolated from a Natural Community. PLoS ONE 8(3): e57139.

Demmig-Adams, B. and W. Adams Iii (1992). Photoprotection and other responses of plants to high light stress. Annual review of plant biology 43(1): 599-626.

Demmig-Adams, B., J. J. Stewart, T. A. Burch and W. W. Adams (2014). Insights from Placing Photosynthetic Light Harvesting into Context. The Journal of Physical Chemistry Letters.

Demmig, B., K. Winter, A. Krüger and F.-C. Czygan (1987). Photoinhibition and zeaxanthin formation in intact leaves a possible role of the xanthophyll cycle in the dissipation of excess light energy. Plant Physiology 84(2): 218-224.

Denny, M. W. (1993). Air and water: the biology and physics of life's media, Princeton University Press.

Doebley, J. F., B. S. Gaut and B. D. Smith (2006). The molecular genetics of crop domestication. Cell 127(7): 1309-1321.

Dubinsky, Z., P. G. Falkowski and K. Wyman (1986). Light harvesting and utilization by phytoplankton. Plant Cell Physiol 27(7): 1335-1349.

Dubinsky, Z. and N. Stambler (2009). Photoacclimation processes in phytoplankton: mechanisms, consequences, and applications. Aquat. Microb. Ecol 56(2-3): 163-176.

Duboc, P., I. Marison and U. Von Stockar (1999). Quantitative calorimetry and biochemical engineering. Handbook of Thermal Analysis and Calorimetry 4: 267-365.

Duffie, J. A. and W. A. Beckman (1980). Solar engineering of thermal processes, Wiley New York etc.

Dufva, M. (2009). Microchips for cell-based assays. Microchip Methods in Diagnostics: 135-144.

Duyens, L. (1956). The flattering of the absorption spectrum of suspensions, as compared to that of solutions. Biochimica et Biophysica Acta 19: 1-12.

Dye, D., J. Muhs, B. Wood and R. Sims (2011). Design and performance of a solar photobioreactor utilizing spatial light dilution. Journal of Solar Energy Engineering 133(1): 015001.

Dye, D. J. (2010). Spatial Light Dilution as a Technique for Conversion of Solar Energy to Algal Biomass. Dissertation, Utah State University.

Emerson, R. and C. M. Lewis (1943). The dependence of the quantum yield of Chlorella photosynthesis on wave lenghth of light. American Journal of Botany: 165-178.

Erickson, E., S. Wakao and K. K. Niyogi (2015). Light stress and photoprotection in Chlamydomonas reinhardtii. The Plant Journal 82(3): 449-465.

Escoubas, J.-M., M. Lomas, J. LaRoche and P. G. Falkowski (1995). Light intensity regulation of cab gene transcription is signaled by the redox state of the plastoquinone pool. Proceedings of the National Academy of Sciences 92(22): 10237-10241.

Evans, J. (1987). The dependence of quantum yield on wavelength and growth irradiance. Functional Plant Biology 14(1): 69-79. 
Fábregas, J., A. Maseda, A. Domínguez, M. Ferreira and A. Otero (2002). Changes in the cell composition of the marine microalga, Nannochloropsis gaditana, during a light: dark cycle. Biotechnology letters 24(20): 1699-1703.

Falkowski, P. G., Z. Dubinsky and K. Wyman (1985). Growth-irradiance relationships in phytoplankton1. Limnology and Oceanography 30(2): 311-321.

Falkowski, P. G. and J. LaRoche (1991). Acclimation to spectral irradiance in algae. Journal of Phycology 27(1): 8-14.

Falkowski, P. G. and J. A. Raven (2013). Aquatic photosynthesis, Princeton University Press.

Flynn, K., A. Mitra, H. Greenwell and J. Sui (2013). Monster potential meets potential monster: pros and cons of deploying genetically modified microalgae for biofuels production. Interface focus 3(1): 20120037.

Flynn, K. J. (2001). A mechanistic model for describing dynamic multi-nutrient, light, temperature interactions in phytoplankton. Journal of Plankton Research 23(9): 977-997.

Flynn, K. J., H. C. Greenwell, R. W. Lovitt and R. J. Shields (2010). Selection for fitness at the individual or population levels: Modelling effects of genetic modifications in microalgae on productivity and environmental safety. Journal of Theoretical Biology 263(3): 269280.

Formighieri, C. (2015). Genetic Modification of the Pigment Optical Density. Solar-to-fuel conversion in algae and cyanobacteria, Springer: 69-82.

Formighieri, C., F. Franck and R. Bassi (2012). Regulation of the pigment optical density of an algal cell: filling the gap between photosynthetic productivity in the laboratory and in mass culture. Journal of biotechnology 162(1): 115-123.

Franco, M. C., M. F. Buffing, M. Janssen, C. V. Lobato and R. H. Wijffels (2012). Performance of Chlorella sorokiniana under simulated extreme winter conditions. Journal of Applied Phycology 24(4): 693-699.

Fröhlich, C. and R. Brusa (1981). Solar radiation and its variation in time. Physics of Solar Variations, Springer: 209-215.

Gangl, D., J. A. Zedler, P. D. Rajakumar, E. M. R. Martinez, A. Riseley, A. Włodarczyk, S. Purton, Y. Sakuragi, C. J. Howe and P. E. Jensen (2015). Biotechnological exploitation of microalgae. Journal of Experimental Botany: erv426.

Ghirardi, M. L., R. K. Togasaki and M. Seibert (1997). Oxygen sensitivity of algal H2-production. Biotechnology for Fuels and Chemicals, Springer: 141-151.

Gibson, R., R. Atkinson and J. Gordon (2008). Use, abuse, misconceptions and insights from quota models - the droop cell quota model 40 years on. Oceanography and Marine Biology: An Annual Review 46: 1-23.

Glick, R. E. and A. Melis (1988). Minimum photosynthetic unit size in system I and system II of barley chloroplasts. Biochimica et Biophysica Acta (BBA)-Bioenergetics 934(1): 151-155.

Goldstein, D. H. (2010). Polarized light, CRC Press.

Gordon, J. M. (2002). Tailoring optical systems to optimized photobioreactors. International journal of hydrogen energy 27(11): 1175-1184. 
Grewe, S., M. Ballottari, M. Alcocer, C. D'Andrea, O. Blifernez-Klassen, B. Hankamer, J. H. Mussgnug, R. Bassi and O. Kruse (2014). Light-Harvesting Complex Protein LHCBM9 Is Critical for Photosystem II Activity and Hydrogen Production in Chlamydomonas reinhardtii. Plant Cell: tpc. 114.124198.

Grima, E. M., J. F. Sevilla, J. S. Perez and F. G. Camacho (1996). A study on simultaneous photolimitation and photoinhibition in dense microalgal cultures taking into account incident and averaged irradiances. Journal of biotechnology 45(1): 59-69.

Grobbelaar, J. U. and N. Kurano (2003). Use of photoacclimation in the design of a novel photobioreactor to achieve high yields in algal mass cultivation. Journal of Applied Phycology 15(2-3): 121-126.

Grossman, A. R., D. Bhaya, K. E. Apt and D. M. Kehoe (1995). Light-harvesting complexes in oxygenic photosynthesis: diversity, control, and evolution. Annual review of genetics 29(1): 231-288.

Gruszecki, W. I., R. Luchowski, M. Zubik, W. Grudzinski, E. Janik, M. Gospodarek, J. Goc, Z. Gryczynski and I. Gryczynski (2010). Blue-light-controlled photoprotection in plants at the level of the photosynthetic antenna complex LHCII. Journal of plant physiology 167(1): 69-73.

Gumpel, N. J., J.-D. Rochaix and S. Purton (1994). Studies on homologous recombination in the green alga Chlamydomonas reinhardtii. Current genetics 26(5-6): 438-442.

Hellingwerf, K. J., W. D. Hoff and W. Crielaard (1996). Photobiology of microorganisms: how photosensors catch a photon to initialize signalling. Molecular microbiology 21(4): 683693.

Hobson, P. (1969). Growth of mixed cultures and their biological control. Microbial Growth: 4364.

Hogewoning, S. W., G. Trouwborst, H. Maljaars, H. Poorter, W. van Ieperen and J. Harbinson (2010). Blue light dose-responses of leaf photosynthesis, morphology, and chemical composition of Cucumis sativus grown under different combinations of red and blue light. Journal of Experimental Botany 61(11): 3107-3117.

Hu, Q., M. Sommerfeld, E. Jarvis, M. Ghirardi, M. Posewitz, M. Seibert and A. Darzins (2008). Microalgal triacylglycerols as feedstocks for biofuel production: perspectives and advances. The Plant Journal 54(4): 621-639.

Huesemann, M. H., T. S. Hausmann, R. Bartha, M. Aksoy, J. C. Weissman and J. R. Benemann (2009). Biomass productivities in wild type and pigment mutant of Cyclotella sp.(Diatom). Appl Biochem Biotechnol 157(3): 507-526.

Huisman, J., R. R. Jonker, C. Zonneveld and F. J. Weissing (1999). Competition for light between phytoplankton species: experimental tests of mechanistic theory. Ecology 80(1): 211222.

Hutner, S., L. Provasoli, A. Schatz and C. Haskins (1950). Some approaches to the study of the role of metals in the metabolism of microorganisms. P Am Philos Soc 94(2): 152-170.

IFA (Accessed Sep 15th, 2015). GESTIS-Stoffdatenbank. 
Jaeger, L. d. (2015). Strain improvement of oleaginous microalgae, Wageningen University.

Janssen, M., M. Janssen, M. de Winter, J. Tramper, L. R. Mur, J. Snel and R. H. Wijffels (2000). Efficiency of light utilization of Chlamydomonas reinhardtii under medium-duration light/dark cycles. J Biotechnol 78(2): 123-137.

Janssen, M., T. C. Kuijpers, B. Veldhoen, M. B. Ternbach, J. Tramper, L. R. Mur and R. H. Wijffels (1999). Specific growth rate of Chlamydomonas reinhardtii and Chlorella sorokiniana under medium duration light/dark cycles: 13-87 s. Journal of biotechnology 70(1): 323333.

Jassby, A. D. and T. Platt (1976). Mathematical formulation of the relationship between photosynthesis and light for phytoplankton. Limnol and Oceanogr 21:540-547.

Johkan, M., K. Shoji, F. Goto, S. Hahida and T. Yoshihara (2012). Effect of green light wavelength and intensity on photomorphogenesis and photosynthesis in Lactuca sativa. Environmental and Experimental Botany 75: 128-133.

Kasten, F. and G. Czeplak (1980). Solar and terrestrial radiation dependent on the amount and type of cloud. Solar Energy 24(2): 177-189.

Kim, C. W., M.-G. Sung, K. Nam, M. Moon, J.-H. Kwon and J.-W. Yang (2014). Effect of monochromatic illumination on lipid accumulation of Nannochloropsis gaditana under continuous cultivation. Bioresource technology 159: 30-35.

Kim, D. G., C. Lee, S.-M. Park and Y.-E. Choi (2014). Manipulation of light wavelength at appropriate growth stage to enhance biomass productivity and fatty acid methyl ester yield using Chlorella vulgaris. Bioresource technology 159: 240-248.

Kim, H.-H., G. D. Goins, R. M. Wheeler and J. C. Sager (2004). Green-light supplementation for enhanced lettuce growth under red-and blue-light-emitting diodes. HortScience 39(7): 1617-1622.

Kim, T.-H., Y. Lee, S.-H. Han and S.-J. Hwang (2013). The effects of wavelength and wavelength mixing ratios on microalgae growth and nitrogen, phosphorus removal using Scenedesmus sp. for wastewater treatment. Bioresource technology 130: 75-80.

Kirst, H., J. G. García-Cerdán, A. Zurbriggen and A. Melis (2012). Assembly of the light-harvesting chlorophyll antenna in the green alga Chlamydomonas reinhardtii requires expression of the TLA2-CpFTSY gene. Plant Physiol 158(2): 930-945.

Klein, U. (1987). Intracellular carbon partitioning in Chlamydomonas reinhardtii. Plant Physiology 85(4): 892-897.

Kliphuis, A. M., L. de Winter, C. Vejrazka, D. E. Martens, M. Janssen and R. H. Wijffels (2010). Photosynthetic efficiency of Chlorella sorokiniana in a turbulently mixed short light-path photobioreactor. Biotechnology progress 26(3): 687-696.

Kliphuis, A. M., M. Janssen, E. J. van den End, D. E. Martens and R. H. Wijffels (2011). Light respiration in Chlorella sorokiniana. Journal of Applied Phycology 23(6): 935-947.

Kliphuis, A. M., A. J. Klok, D. E. Martens, P. P. Lamers, M. Janssen and R. H. Wijffels (2012). Metabolic modeling of Chlamydomonas reinhardtii: energy requirements for photoautotrophic growth and maintenance. J Appl Phycol 24(2): 253-266. 
Kubín, Š., E. Borns, J. Doucha and U. Seiss (1983). Light Absorption and Production Rate of $<\mathrm{i}>$ Chlorella vulgaris $</ \mathrm{i}>$ in Light of Different Spectral Composition. Biochemie und Physiologie der Pflanzen 178(2): 193-205.

Kwon, J.-H., G. Bernát, H. Wagner, M. Rögner and S. Rexroth (2013). Reduced light-harvesting antenna: Consequences on cyanobacterial metabolism and photosynthetic productivity. Algal Research 2(3): 188-195.

Lacour, T., A. Sciandra, A. Talec, P. Mayzaud and O. Bernard (2012). Diel variations of carbohydrates and neutral lipids in nitrogen-sufficient and nitrogen-starved cyclostat cultures of isochrysis sp. 1. Journal of Phycology 48(4): 966-975.

Lambrev, P. H., M. Nilkens, Y. Miloslavina, P. Jahns and A. R. Holzwarth (2010). Kinetic and spectral resolution of multiple nonphotochemical quenching components in Arabidopsis leaves. Plant Physiology 152(3): 1611-1624.

Lee, K. S., P. Boccazzi, A. J. Sinskey and R. J. Ram (2011). Microfluidic chemostat and turbidostat with flow rate, oxygen, and temperature control for dynamic continuous culture. Lab on a Chip 11(10): 1730-1739.

Lee, Y.-K., S.-Y. Ding, C.-H. Hoe and C.-S. Low (1996). Mixotrophic growth ofChlorella sorokiniana in outdoor enclosed photobioreactor. Journal of Applied Phycology 8(2): 163-169.

Li, T., B. Podola and M. Melkonian (2016). Investigating dynamic processes in a porous substrate biofilm photobioreactor-A modeling approach. Algal Research 13: 30-40.

Li, Z., S. Wakao, B. B. Fischer and K. K. Niyogi (2009). Sensing and responding to excess light. Annual review of plant biology 60: 239-260.

Liberton, M., A. M. Collins, L. E. Page, W. B. O’Dell, H. O’Neill, V. S. Urban, J. A. Timlin and H. B. Pakrasi (2013). Probing the consequences of antenna modification in cyanobacteria. Photosynthesis Res 118(1-2): 17-24.

Logan, B. A., W. W. Adams and B. Demmig-Adams (2007). Viewpoint: Avoiding common pitfalls of chlorophyll fluorescence analysis under field conditions. Functional Plant Biology 34(9): 853-859.

MacIntyre, H. L., T. M. Kana, T. Anning and R. J. Geider (2002). Photoacclimation of photosynthesis irradiance response curves and photosynthetic pigments in microalgae and cyanobacteria1. Journal of Phycology 38(1): 17-38.

Malkin, S. and D. C. Fork (1996). Bill Arnold and calorimetric measurements of the quantum requirement of photosynthesis-once again ahead of his time. Photosynthesis research 48(1-2): 41-46.

Manandhar-Shrestha, K. and M. Hildebrand (2013). Development of flow cytometric procedures for the efficient isolation of improved lipid accumulation mutants in a Chlorella sp. microalga. Journal of Applied Phycology 25(6): 1643-1651.

Masojídek, J., G. Torzillo, M. Koblížek, J. Kopecký, P. Bernardini, A. Sacchi and J. Komenda (1999). Photoadaptation of two members of the Chlorophyta (Scenedesmus and Chlorella) in laboratory and outdoor cultures: changes in chlorophyll fluorescence quenching and the xanthophyll cycle. Planta 209(1): 126-135. 
Matsuda, R., K. Ohashi-Kaneko, K. Fujiwara and K. Kurata (2008). Effects of blue light deficiency on acclimation of light energy partitioning in PSII and CO2 assimilation capacity to high irradiance in spinach leaves. Plant and cell physiology 49(4): 664-670.

Mattos, E. R., M. Singh, M. L. Cabrera and K. C. Das (2015). Enhancement of biomass production in Scenedesmus bijuga high-density culture using weakly absorbed green light. Biomass and Bioenergy 81: 473-478.

Maxwell, K. and G. N. Johnson (2000). Chlorophyll fluorescence-a practical guide. Journal of Experimental Botany 51(345): 659-668.

Meinhold, T., J.-P. Richters, L. Damerow and M. Blanke (2010). Optical properties of reflection ground covers with potential for enhancing fruit colouration. Biosystems engineering 107(2): 155-160.

Melis, A. (1999). Photosystem-II damage and repair cycle in chloroplasts: what modulates the rate of photodamage in vivo? Trends Plant Sci 4(4): 130-135.

Melis, A. (2009). Solar energy conversion efficiencies in photosynthesis: minimizing the chlorophyll antennae to maximize efficiency. Plant science 177(4): 272-280.

Melis, A., J. Neidhardt and J. R. Benemann (1998). Dunaliella salina (Chlorophyta) with small chlorophyll antenna sizes exhibit higher photosynthetic productivities and photon use efficiencies than normally pigmented cells. Journal of Applied Phycology 10(6): 515-525.

Mendoza, H., A. de la Jara, K. Freijanes, L. Carmona, A. A. Ramos, V. de Sousa Duarte, S. Varela and J. Carlos (2008). Characterization of Dunaliella salina strains by flow cytometry: a new approach to select carotenoid hyperproducing strains. Electronic Journal of Biotechnology 11(4): 5-6.

Michels, M. H., A. J. van der Goot, N.-H. Norsker and R. H. Wijffels (2010). Effects of shear stress on the microalgae Chaetoceros muelleri. Bioprocess and biosystems engineering 33(8): 921-927.

Mitra, M. and A. Melis (2008). Optical properties of microalgae for enhanced biofuels production. Optics express 16(26): 21807-21820.

Mooij, P. R., G. R. Stouten, M. C. van Loosdrecht and R. Kleerebezem (2015). Ecology-based selective environments as solution to contamination in microalgal cultivation. Current opinion in biotechnology 33: 46-51.

Morel, A. and A. Bricaud (1981). Theoretical results concerning light absorption in a discrete medium, and application to specific absorption of phytoplankton. Deep Sea Research Part A. Oceanographic Research Papers 28(11): 1375-1393.

Müller, P., X.-P. Li and K. K. Niyogi (2001). Non-photochemical quenching. A response to excess light energy. Plant Physiology 125(4): 1558-1566.

Münzner, P. and J. Voigt (1992). Blue light regulation of cell division in Chlamydomonas reinhardtii. Plant Physiology 99(4): 1370-1375.

Mussgnug, J. H., S. Thomas-Hall, J. Rupprecht, A. Foo, V. Klassen, A. McDowall, P. M. Schenk, O. Kruse and B. Hankamer (2007). Engineering photosynthetic light capture: impacts on improved solar energy to biomass conversion. Plant Biotechnol J 5(6): 802-814. 
Nakajima, Y., M. Tsuzuki and R. Ueda (2001). Improved productivity by reduction of the content of light-harvesting pigment in Chlamydomonas perigranulata. J Appl Phycol 13(2): 95101.

Nakajima, Y. and R. Ueda (2000). The effect of reducing light-harvesting pigment on marine microalgal productivity. J Appl Phycol 12(3-5): 285-290.

Neidhardt, J., J. R. Benemann, L. Zhang and A. Melis (1998). Photosystem-II repair and chloroplast recovery from irradiance stress: relationship between chronic photoinhibition, light-harvesting chlorophyll antenna size and photosynthetic productivity in Dunaliella salina (green algae). Photosynthesis research 56(2): 175-184.

Norsker, N.-H., M. J. Barbosa, M. H. Vermuë and R. H. Wijffels (2011). Microalgal production-a close look at the economics. Biotechnology Advances 29(1): 24-27.

Oey, M., I. L. Ross, E. Stephens, J. Steinbeck, J. Wolf, K. A. Radzun, J. Kügler, A. K. Ringsmuth, O. Kruse and B. Hankamer (2013). RNAi knock-down of LHCBM1, 2 and 3 increases photosynthetic $\mathrm{H} 2$ production efficiency of the green alga Chlamydomonas reinhardtii. PLoS ONE 8(4): e61375.

Ogbonna, J. C. and H. Tanaka (1996). Night biomass loss and changes in biochemical composition of cells during light/dark cyclic culture of Chlorella pyrenoidosa. Journal of Fermentation and Bioengineering 82(6): 558-564.

Oldenhof, H., V. Zachleder and H. Van Den Ende (2006). Blue-and red-light regulation of the cell cycle in Chlamydomonas reinhardtii (Chlorophyta). European Journal of Phycology 41(3): 313-320.

Olive, J., F.-A. Wollman, P. Bennoun and M. Recouvreur (1981). Ultrastructure of thylakoid membranes in C. reinhardtii: Evidence for variations in the partition coefficient of the light-harvesting complex-containing particles upon membrane fracture. Arch Biochem Biophys 208(2): 456-467.

Olle, M. and A. Viršile (2013). The effects of light-emitting diode lighting on greenhouse plant growth and quality. Agricultural and Food Science 22(2): 223-234.

Ort, D. R., X. Zhu and A. Melis (2011). Optimizing antenna size to maximize photosynthetic efficiency. Plant physiology 155(1): 79-85.

Paradiso, R., E. Meinen, J. F. Snel, P. De Visser, W. Van Ieperen, S. W. Hogewoning and L. F. Marcelis (2011). Spectral dependence of photosynthesis and light absorptance in single leaves and canopy in rose. Scientia Horticulturae 127(4): 548-554.

Peers, G., T. B. Truong, E. Ostendorf, A. Busch, D. Elrad, A. R. Grossman, M. Hippler and K. K. Niyogi (2009). An ancient light-harvesting protein is critical for the regulation of algal photosynthesis. Nature 462(7272): 518-521.

Perin, G., A. Bellan, A. Segalla, A. Meneghesso, A. Alboresi and T. Morosinotto (2015). Generation of random mutants to improve light-use efficiency of Nannochloropsis gaditana cultures for biofuel production. Biotechnology for biofuels 8(1): 1.

Perrine, Z., S. Negi and R. T. Sayre (2012). Optimization of photosynthetic light energy utilization by microalgae. Algal Res 1:134-142. 
Pirt, S. (1965). The maintenance energy of bacteria in growing cultures. Proceedings of the Royal Society of London. Series B. Biological Sciences 163(991): 224-231.

Polle, J. E., S.-D. Kanakagiri and A. Melis (2003). tla1, a DNA insertional transformant of the green alga Chlamydomonas reinhardtii with a truncated light-harvesting chlorophyll antenna size. Planta 217(1): 49-59.

Post, A. F., J. G. loogman and L. R. Mur (1985). Regulation of growth and photosynthesis by Oscillatoria agardhii grown with a light/dark cycle. FEMS Microbiology Letters 31(2): 97102.

Pruvost, J., J. Cornet, F. Le Borgne, V. Goetz and J. Legrand (2015). Theoretical investigation of microalgae culture in the light changing conditions of solar photobioreactor production and comparison with cyanobacteria. Algal Research 10: 87-99.

Pulz, O. and W. Gross (2004). Valuable products from biotechnology of microalgae. Applied microbiology and biotechnology 65(6): 635-648.

Radmer, R. and B. Kok (1977). Photosynthesis: limited yields, unlimited dreams. Bioscience 27(9): 599-605.

Raso, S., B. Van Genugten, M. Vermuë and R. H. Wijffels (2012). Effect of oxygen concentration on the growth of Nannochloropsis sp. at low light intensity. Journal of Applied Phycology 24(4): 863-871.

Rosenberg, J. N., N. Kobayashi, A. Barnes, E. A. Noel, M. J. Betenbaugh and G. A. Oyler (2014). Comparative analyses of three Chlorella species in response to light and sugar reveal distinctive lipid accumulation patterns in the microalga C. sorokiniana. PLOS ONE 9(4): e92460.

Schaap, A., T. Rohrlack and Y. Bellouard (2012). Optical classification of algae species with a glass lab-on-a-chip. Lab on a Chip 12(8): 1527-1532.

Schulze, P. S., L. A. Barreira, H. G. Pereira, J. A. Perales and J. C. Varela (2014). Light emitting diodes (LEDs) applied to microalgal production. Trends in biotechnology 32(8): 422-430.

Sforza, E., E. Barbera and A. Bertucco (2015). Improving the photoconversion efficiency: An integrated photovoltaic-photobioreactor system for microalgal cultivation. Algal Research 10: 202-209.

Sforza, E., C. Calvaruso, A. Meneghesso, T. Morosinotto and A. Bertucco (2015). Effect of specific light supply rate on photosynthetic efficiency of Nannochloropsis salina in a continuous flat plate photobioreactor. Applied microbiology and biotechnology: 1-10.

Sharkey, T. D. (2015). Understanding carbon partitioning and its role in determining plant growth. Plant Cell Environ.

Siefermann-Harms, D. (1985). Carotenoids in photosynthesis. I. Location in photosynthetic membranes and light-harvesting function. Biochimica et Biophysica Acta (BBA)-Reviews on Bioenergetics 811(4): 325-355.

Solovchenko, A. (2010). Photoprotection in plants: optical screening-based mechanisms, Springer Science \& Business Media. 
Sonnewald, U. and J. Kossmann (2013). Starches-from current models to genetic engineering. Plant biotechnology journal 11(2): 223-232.

Sorokin, C. and J. Myers (1953). A high-temperature strain of Chlorella. Carnegie Institution of Washington. Yearbook No. 51: 141-142.

Sousa, C., A. Compadre, M. H. Vermuë and R. H. Wijffels (2013). Effect of oxygen at low and high light intensities on the growth of Neochloris oleoabundans. Algal Research 2(2): 122126.

Sukenik, A., J. Bennett and P. Falkowski (1987). Light-saturated photosynthesis-limitation by electron transport or carbon fixation? Biochimica et Biophysica Acta (BBA)-Bioenergetics 891(3): 205-215.

Sukenik, A., J. Bennett, A. Mortain-Bertrand and P. G. Falkowski (1990). Adaptation of the photosynthetic apparatus to irradiance in Dunaliella tertiolecta A Kinetic Study. Plant Physiology 92(4): 891-898.

Sukenik, A. and Y. Carmeli (1990). Lipid synthesis and fatty acid composition in Nannochloropsis sp. (eustigmatophyceae grown in a light-dark cycle1. Journal of Phycology 26(3): 463469.

Takache, H., G. Christophe, J. F. Cornet and J. Pruvost (2010). Experimental and theoretical assessment of maximum productivities for the microalgae Chlamydomonas reinhardtii in two different geometries of photobioreactors. Biotechnology progress 26(2): 431-440.

Takache, H., J. Pruvost and J. F. Cornet (2012). Kinetic modeling of the photosynthetic growth of Chlamydomonas reinhardtii in a photobioreactor. Biotechnology progress 28(3): 681692.

Tanada, T. (1951). The photosynthetic efficiency of carotenoid pigments in Navicula minima. American Journal of Botany: 276-283.

Tanaka, R. and A. Tanaka (2000). Chlorophyll b is not just an accessory pigment but a regulator of the photosynthetic antenna. Porphyrins 9: 240-245.

Terashima, I., T. Fujita, T. Inoue, W. S. Chow and R. Oguchi (2009). Green light drives leaf photosynthesis more efficiently than red light in strong white light: revisiting the enigmatic question of why leaves are green. Plant and cell physiology 50(4): 684-697.

Tokutsu, R., M. Iwai and J. Minagawa (2009). CP29, a monomeric light-harvesting complex II protein, is essential for state transitions in Chlamydomonas reinhardtii. J Biol Chem 284(12): 7777-7782.

Tokutsu, R. and J. Minagawa (2013). Energy-dissipative supercomplex of photosystem II associated with LHCSR3 in Chlamydomonas reinhardtii. P Natl Acad Sci USA 110(24): 10016-10021.

Tredici, M. R. and G. C. Zittelli (1998). Efficiency of sunlight utilization: tubular versus flat photobioreactors. Biotechnology and bioengineering 57(2): 187-197.

Treves, H., H. Raanan, I. Kedem, O. Murik, N. Keren, H. Zer, S. M. Berkowicz, M. Giordano, A. Norici and Y. Shotland (2016). The mechanisms whereby the green alga Chlorella ohadii, isolated from desert soil crust, exhibits unparalleled photodamage resistance. New Phytologist. 
Van Wagenen, J., S. L. Holdt, D. De Francisci, B. Valverde-Pérez, B. G. Plósz and I. Angelidaki (2014). Microplate-based method for high-throughput screening of microalgae growth potential. Bioresource technology 169: 566-572.

Vanthoor-Koopmans, M., R. H. Wijffels, M. J. Barbosa and M. H. Eppink (2013). Biorefinery of microalgae for food and fuel. Bioresource technology 135: 142-149.

Vejrazka, C., M. Janssen, G. Benvenuti, M. Streefland and R. H. Wijffels (2013). Photosynthetic efficiency and oxygen evolution of Chlamydomonas reinhardtii under continuous and flashing light. Applied microbiology and biotechnology 97(4): 1523-1532.

Velds, C. A., P. Van der Hoeven, J. Koopstra, W. Raaf and W. Slob (1992). Zonnestraling in Nederland, Thieme Baarn.

Wang, J., J. Liu and T. Liu (2015). The difference in effective light penetration may explain the superiority in photosynthetic efficiency of attached cultivation over the conventional open pond for microalgae. Biotechnology for biofuels 8(1): 49.

Wang, S. K., A. R. Stiles, C. Guo and C. Z. Liu (2014). Microalgae cultivation in photobioreactors: An overview of light characteristics. Engineering in Life Sciences 14(6): 550-559.

Wijffels, R. H., O. Kruse and K. J. Hellingwerf (2013). Potential of industrial biotechnology with cyanobacteria and eukaryotic microalgae. Current opinion in biotechnology 24(3): 405413.

Wilhelm, C., P. Krámer and A. Wild (1985). Effect of different light qualities on the ultrastructure, thylakoid membrane composition and assimilation metabolism of Chlorella fusca. Physiologia Plantarum 64(3): 359-364.

Wobbe, L., R. Bassi and O. Kruse (2016). Multi-Level Light Capture Control in Plants and Green Algae. Trends in plant science 21(1): 55-68.

Young, E. B. and J. Beardall (2003). Photosynthetic function in Dunaliella tertiolecta (Chlorophyta) during a nitrogen starvation and recovery cycle. Journal of Phycology 39(5): 897-905.

Zemke, P. E., M. R. Sommerfeld and Q. Hu (2013). Assessment of key biological and engineering design parameters for production of Chlorella zofingiensis (Chlorophyceae) in outdoor photobioreactors. Appl Microbiol Biotechnol 97(12): 5645-5655.

Zhu, X.-G., E. de Sturler and S. P. Long (2007). Optimizing the distribution of resources between enzymes of carbon metabolism can dramatically increase photosynthetic rate: a numerical simulation using an evolutionary algorithm. Plant Physiology 145(2): 513-526.

Zhu, X.-G., S. P. Long and D. R. Ort (2010). Improving photosynthetic efficiency for greater yield. Annual review of plant biology 61: 235-261.

Zijffers, J.-W. F., M. Janssen, J. Tramper and R. H. Wijffels (2008). Design process of an areaefficient photobioreactor. Marine Biotechnology 10(4): 404-415.

Zijffers, J.-W. F., K. J. Schippers, K. Zheng, M. Janssen, J. Tramper and R. H. Wijffels (2010). Maximum photosynthetic yield of green microalgae in photobioreactors. Marine Biotechnology 12(6): 708-718. 
Summary 
Microalgae have great potential to become a sustainable source of food, feed and fuel in a biobased economy. To obtain cost-effective production processes, it is crucial that the photosynthetic efficiency achieved in microalgae mass cultures is improved. Microalgae have the ability to perform photosynthesis at a high efficiency under low light conditions, but due to their relatively high chlorophyll content, they are easily oversaturated after exposure to full sunlight. Microalgal cells then absorb more light energy than can be converted to biochemical energy, which oversaturates their photosynthetic machinery. Oversaturation leads to dissipation of the excess light energy absorbed, which is a wasteful process that decreases the overall light use efficiency. Reduction of the chlorophyll content by means of genetic engineering is a promising biological solution to oversaturation. The obtained antenna size mutants are cells that are more transparent and in theory less prone to oversaturation by sunlight. Because antenna size mutants absorb less light per cell, conversion of solar energy to biochemical energy is expected to occur at a higher efficiency. The aim of this thesis was to evaluate the potential of antenna size reduction in order to increase the biomass productivity in microalgae mass cultures. This evaluation of antenna reduction technology is supported by a large experimental dataset obtained under well-defined simulated mass culture conditions, and model-based estimations of the gain in productivity that can realistically be expected for cultivation outdoors.

Long-term photobioreactor experiments with both antenna size mutants of Chlamydomonas reinhardtii and the wild type were presented In Chapter 2 . The experiments were performed under turbidostat control and a well-defined light regime. These conditions provided a reliable basis to study the biomass productivity under mass culture conditions, which are defined as a fixed incident light intensity of $1500 \mu \mathrm{mol}$ photons $\mathrm{m}^{-2} \mathrm{~s}^{-1}$, and a biomass concentration high enough to virtually absorb all the incident light. Four promising antenna size mutants were assessed for their maximal productivity. The mutants, however, did not exhibit the expected increase in biomass productivity under mass culture conditions. The highest mutant productivity values were comparable $\left(43-50 \mathrm{~g} \mathrm{~m}^{-2} \mathrm{~d}^{-1}\right)$ to that of wild-type productivity $\left(46 \mathrm{~g} \mathrm{~m}^{-2} \mathrm{~d}^{-1}\right)$. The biomass yield on light energy ranged between 0.33 and $0.41 \mathrm{~g} \mathrm{~mol}_{\mathrm{ph}^{-1}}$ for the mutants, and was $0.36 \mathrm{~g} \mathrm{~mol}_{\mathrm{ph}^{-1}}$ for the wild-type strain. Observations indicated a reduced fitness of the mutant cultures caused by a high light intensity. This might be explained by a 
higher susceptibility to photodamage, possibly induced by the antenna complex alterations, or by unintended side effects of the genetic modifications. It is suggested that more emphasis must be placed on selecting mutants with a higher tolerance to high light conditions.

Utilizing a wild-type Chlamydomonas strain, a proof of principle was delivered that the biomass productivity in a mass culture depends on the light absorbing capacity of microalgae cells. In this study (Chapter 3), artificial light of different colors was employed as a tool to generate different specific light absorption rates by spectral tuning. The biomass productivity of cultures exposed to warm-white, orange-red, deepred, blue and yellow light was measured, and turbidostat control ensured that the total amount of absorbed light was equal for each color. The results demonstrated that, under mass culture conditions, biomass productivity and the biomass specific light absorption rate are inversely correlated. Highest biomass productivity was obtained using yellow light (54 $\left.\mathrm{g} \mathrm{m}^{-2} \mathrm{~d}^{-1}\right)$, closely followed by cultures grown under warm white light $\left(50 \mathrm{~g} \mathrm{~m}^{-2}\right.$ $\mathrm{d}^{-1}$ ). Cultivation under blue, orange-red, and deep red light resulted in biomass productivities of approximately $29 \mathrm{~g} \mathrm{~m}^{-2} \mathrm{~d}^{-1}$ which is nearly half of the productivity measured for yellow light. Apart from the effect different light colors on oversaturation, the experiments were interfered by intrinsic biological effects. Cultivation under yellow light was impeded but minimal supplementation of blue light was sufficient to stimulate normal growth and maximize productivity. It was discussed in more detail whether cultivation of microalgae on monochromatic light could cause a spectral deficiency.

In Chapter 4 it was attempted to simulate the effect of reduced antenna size on mass culture productivity by acclimating wild type cells of Chlorella sorokiniana to high light conditions. The resulting low-pigmented cells were consequently exposed to mass culture conditions and rapidly analyzed for their biomass productivity before they returned to their normal state with a high pigment content. In a first approach, lowpigmented cells were continuously pumped into a photobioreactor operated under mass culture conditions. The cell retention time was two hours, which was enough to maintain a high light phenotype. The productivity of this culture was measured on a daily basis. In a second approach, a biological oxygen monitor (BOM) was operated under mass culture conditions. Low-pigmented cells were transferred from a high light 
exposed photobioreactor to the BOM and the volumetric oxygen production rate was measured for several minutes and compared to the oxygen production of normally pigmented cells. The combination of both approaches provided insight in the short-term (minutes) and longer-term (hours) response of high light acclimated cells upon cultivation in a mass culture. In addition, a light shift-down experiment was performed to get an understanding of pigment accumulation kinetics. Based on model estimations, a low-pigmented culture is expected to express a higher productivity because of reduced oversaturation. However, in both experiments no productivity improvement was observed in comparison to normally pigmented cells. The most plausible explanation is that the high light phenotype came along with an increased capacity and tendency to thermally dissipate light energy. A second explanation might be that a considerable amount of energy was spent on the photoacclimation process itself, thereby counterbalancing the theoretical gain in light use efficiency that was expected upon reducing the optical cross section. The results demonstrate that high light acclimated cells do not increase productivity under mass culture conditions. It is suggested though that for growth of microalgae in biofilms, local acclimation might be beneficial because microalgae will maintain their high-light phenotype.

A theoretical model was presented In Chapter 5 to simulate cultivations of antenna size mutants and the competition with their wild type under mass culture conditions. The model is based on experimental data from wild type $C$. sorokiniana cultivations and takes the spectral dependency of photosynthesis, the effect of day / night cycles, and carbon partitioning between functional biomass and storage carbohydrates into account. It was found that for a Mediterranean location (Tunisia) the increase in biomass productivity of Chlorella sorokiniana with antenna size reduction is about $40 \%$ while for the Netherlands the increase was only $16 \%$. It was therefore concluded that a location of high irradiance is required to obtain a considerable increase in productivity using antenna size reduction technology. An unexpected new factor that was limiting the productivity was the inability of the mutants to absorb all incident sunlight due to their limited light uptake rate. As a consequence, at high antenna size reduction, a significant fraction of the light passed through the reactor without being absorbed. By increasing the biomass concentration, more light is absorbed, but this increases the maintenances costs, which also limits productivity. The model predicted that hypothetical mutants that 
are only reduced in their absorption cross section, will always lose the competition for light with their wild type. At the lowest contamination level, the monocultures were overgrown within 27 days. With increasing contamination level and with increasing antenna size reduction, the mutants were overgrown in an earlier stage and this substantially decreased the gain in productivity that could be obtained using antenna size mutants over the entire simulated summer period of 100 days. It was concluded that the competitiveness of antenna size mutants needs to be improved in order to obtain strains that have industrial potential.

The last chapter (Chapter 6) is a general discussion of the presented results and the conclusions that were drawn in this thesis. First oversaturation of photosynthesis was addressed and it was concluded that it represents a problem that cannot be solved easily under mass culture conditions. It was explained that domesticating microalgae via genetic engineering is required to obtain commercially interesting strains in the future. Antenna size mutant creation methods and the possible implications for the fitness of the cell were discussed. A final evaluation was made on the potential and limitations of the antenna size reduction technology. Special attention was paid to the issue of how to improve the reduced competitiveness of antenna size mutants under mass culture conditions. Finally a novel mutant selection strategy was proposed that could yield more viable mutants that are better prepared for mass culture conditions. 
Dankwoord 
$\mathrm{Na}$ vier jaar onderzoek doen, algen kweken, artikelen schrijven, onderwijs geven, cursussen volgen en symposia bezoeken, kijk ik vooral terug op een heel erg leuke en gezellige tijd bij Bioprocess Engineering en de Wageningen Universiteit. Ik wil dan ook heel graag van de gelegenheid gebruik maken om iedereen die, direct of indirect, heeft bijgedragen aan dit proefschrift en deze bijzondere periode in mijn leven, te bedanken.

Laat ik beginnen met mijn begeleiders. René, bedankt dat ik de kans heb gekregen voor dit promotietraject in de algenwereld, ik heb het al die tijd als een voorrecht gezien om BPE te mogen vertegenwoordigen en mijn steentje bij te kunnen dragen. Ik wil je specifiek bedanken voor je vaak hele directe feedback op mijn manuscripten, die daar gelukkig veel korter en bondiger van werden. Ik heb er altijd versteld van gestaan dat je tijdens onze meetings vliegtickets aan het boeken was, e-mails afhandelde, maar tegelijkertijd met heel scherp en helder commentaar wist te komen, waaruit bleek dat je enorm betrokken en efficiënt was. Marcel, ik ben je heel dankbaar voor alle handvatten die jij me gegeven hebt bij mijn onderzoek. Het is zowel op het persoonlijke als het professionele vlak heel leerzaam geweest hoe je mij begeleid hebt. Bedankt voor alle kansen, vrijheid, het vertrouwen, de aanmoediging en de in mijn ogen onuitputtelijke energie die je hebt om verbeteringen aan te blijven voeren in manuscripten. Ik heb het lastig gevonden dat onze mutanten hun werk niet goed deden. Het was fijn dat je me hielp om dat te relativeren. Bedankt ook voor de indrukwekkende reis naar Israël die we samen maakten, dat je me in het ziekenhuis in Ede in een rolstoel hebt vervoerd (onder mijn protest) en in het algemeen voor een luisterend oor en gezelligheid in de pauzes.

Veel van mijn laboratoriumwerk is uitgevoerd door MSc en BSc studenten en daarom ben ik hen ook heel erg dankbaar. Oscar, you were my first student and I think I was even more nervous than you were on your first day at BPE. You did great work and I am very pleased that you are co-author of my first paper. Guus, wat heb jij hard gewerkt in de weekenden, gelukkig maar dat dit ook voor jou een welverdiende publicatie heeft opgeleverd! Je had je reistijd naar het Biotechnion wel wat kunnen verkorten door een keer je fietsband te plakken. Christos, you were so extremely dedicated to your labwork, that we had to force you to stop. I am confident that this perseverance will be of great value in your future career. Lennard, bedankt voor het uitwerken van een nieuwe experimentele opstelling, die ik zelf later nog gebruikt heb! Zeynab, bedankt voor het vele en zeer nauwkeurige labwerk dat je hebt gedaan met de BOM! We hebben samen ook veel bijzondere momenten mee gemaakt. Het was wat ongemakkelijk toen we samen in het donker in het damestoilet waren en er iemand verbaasd binnen kwam. De (elektriciteit)vonken sprongen er vanaf haha. Alles voor de wetenschap! Veel succes met je nieuwe baan en laten we snel weer afspreken! Laurens, jammer dat de Chlorella mutant ons geen succes opleverde ondanks al je inspanning. Dat hoort ook bij wetenschap. Ik bewonderde bij jou erg je innovativiteit en het denken buiten de gebaande paden. Dat gaat je zeker verder brengen. Succes met het afronden van je MSc! Kira, thanks for your enormous contribution to the model paper. I'm very proud of our joint effort. You deserve the shared $1^{\text {st }}$ co-authorship more than anyone else. Your energy and devotion makes me feel jealous. I wish you all the best in your own PhD project in Karlsruhe!

Fred en Sebastiaan, bedankt voor het helpen ombouwen van mijn GMO-lab in het Biotechnion en dat jullie altijd klaar stonden bij acute reactorproblemen of spoedbestellingen. Ik zal ook zeker de gezellige gesprekken missen tussen de bedrijven door! De mannen van de werkplaats, met name Reinoud, Hans en Hans, bedankt voor de 
grote hulp bij het (verder) ontwikkelen van de Algaemist photobioreactoren en voor allerlei andere problemen, storingen, verloopjes en software updates.

Richard, ten eerste vind ik het erg leuk dat je mijn paranimf wilt zijn! Wij hebben erg veel meegemaakt samen en het was fijn om altijd iemand te hebben om de dagelijkse ballast kwijt te kunnen, vaak tijdens het fietsen naar onze woonplaats Arnhem of station EdeWageningen. In Bennekom is tegenwoordig de rust weer terug, nu onze 'offensieve' woonwerk verkeer fietstochten tot het verleden behoren. Ik heb nog een trauma van ons optreden als Sick and Nimon op het pink party kerstdiner, maar onze levensgrote kartonnen Rosanne was wel prachtig! Onze gezamenlijke erfenis voor BPE, tot op de dag van vandaag, is een heuse kleedkamer voor fietsers. Dat de schoonmakers niet doorhadden dat dit een 1-april grap was, en er vervolgens een bord met 'kleedkamerregelement' ophingen, maakte dat alleen nog maar mooier. Ons er-is-nog-taart-over alarmeer systeem was ook erg efficiënt. Succes met je eigen laatste loodjes! Merel en Stijn, ook jullie bedankt voor de leuke dagjes en avonden in Arnhem!

Laura, mijn andere paranimf. De vriendschap met jou gaat als een rode draad door mijn $\mathrm{PhD}$. Bij jou kon ik al mijn persoonlijke problemen kwijt en je hebt altijd goede adviezen. Ook nadat onze afdelingen verhuisden, bleven we party-crashen op elkaars feestjes. Fijn ook dat ik mijn eigen kamertje had in jouw huis. Een ideale uitvalsplaats als het te laat was geworden na het stappen. Ik blijf me verzetten, maar nu ik zelf in Brabant werk, zal het qua taal wel even wennen 'worre'. Da is! Bedankt dat jij er altijd voor me bent als het nodig is! Heel erg veel plezier op je grote reis die je gaat maken 2 dagen na mijn verdediging!

Kim, bedankt voor hilarische koffiepauzes, wat kun jij mensen blij maken met slechts je aanwezigheid en trappelende voetjes. En wat was het makkelijk om voor jou een 'stukje' te maken. Ik heb altijd het gevoel gehad dat wij elkaar goed begrepen en daar nemen we ook nu nog goed de tijd voor met ons wekelijkse telefoongesprek, uiteraard strak gepland om 20:00 op donderdag. Laten we die er in houden! Bedankt voor al je inhoudelijke maar ook mentale hulp! Lenneke, jij was het brandende vuurtje voor de gezelligheid bij BPE door bijna alle events te organiseren. Je gaf mij, met je eigen ervaring, ook veel houvast tijdens de gebruikelijke PhD-dipjes. Bedankt voor je betrokkenheid, optimisme, aanmoediging en al de borrels natuurlijk. Grappig dat we nu allebei vanuit de algjes in de pharma beland zijn! Ik denk ook met veel plezier terug aan onze mini-fietsvakantie met friet van Aniet, pontje Steur en met een noodvaart over de afsluitdijk! Snel weer een keertje een fietstocht inplannen? Guido, bedankt voor de talloze gezellige pauzes, borrels en het delen van je kennis. Super dat je ook bij MSD bent komen werken en weer in de buurt bent komen wonen. Fijn dat ik bij jouw verdediging al even mocht proefdraaien door op het podium te zitten als paranimf. João van der Zee, Mr. Avocado with propeller hat, thanks for being who you are! You have amazed and inspired me so many times and it has been a real pleasure to work with you the past years. Live life to the fullest! Anne, van jou heb ik veel geleerd tijdens mijn MSc thesis. Algen- en reactortechniekjes maar ook hoe te handelen in bepaalde situaties. Gaaf dat we later collega's werden en ik je af en toe nog eens aan je jas kon trekken. Bedankt voor al je enthousiasme! Agi, working in your lab was a challenge sometimes, but your presence in the lab changed the atmosphere, thanks for your happiness, positivism and lab-talk! I still wonder why your lab coat was always dirtier than mine... Ward, bedankt voor het uitlenen van je algen, maar ook van je slaapbank na een avond stappen in Wageningen. Ik kan het begrijpen als dat laatste niet voor herhaling vatbaar is. Succes met je verdediging de week voor mij! Ana S., thanks for our conversations in the lab in which we could share our frustrations about crashing reactors and shitty algae performance, I hope you are doing well! Wish you all the best! Ilse, wij hebben veel in het zelfde lab gewerkt, ook in de weekenden. Bedankt voor de goede 
efficiënte samenwerking en dat je altijd bereid was om te helpen. Bedankt ook voor de 'sociale cohesie' waar je voor zorgde in de groep door bijvoorbeeld het in stand houden van de home made soup/dish traditie e.d. Stefan, bedankt voor de nuchtere en humorvolle gesprekken! Jeroen, bedankt voor hulp met reactor-handigheidjes en het organiseren van vele borrels en voor het zijn van de grill master! Kevin, bedankt voor de goede feestjes en de kotsfilmpjes. Leuk om er nog steeds zo nu en dan nog een te vinden in mijn mailbox. Dixi na het stokbrood blijft mijn favoriet. Succes bij NIZO! Wendy, bedankt voor het luisterende oor, je inzet voor uitjes en natuurlijk voor mijn persoonlijke verzoeknummer: de Titanictaart!! Bregje, bedankt voor de leuke fietstochten naar Arnhem. Jij elektrisch en ik buiten adem ... bij mijn verdediging dan eindelijk maar mijn nieuwe baan vieren? Snezana, bedankt voor de gezelligheid en je bijzonder lekkere taarten! Arjen, bedankt voor je betrokkenheid, interessante gesprekken maar ook grappen en grollen in de Wit's koffiezit.

Guido, Kim, Lenneke, Anne, Lenny and Marjon. Ook de Young-ste Algaeneer is groot geworden. Bedankt dat we samen ons geweldige symposium hebben mogen organiseren. Ik lig nog dubbel als ik aan de scientific-speed-date-in-pink denk!

Kiira, Pauline and Xiao, thanks for letting me be part of the most awesome BPE office! Thanks for sharing (and letting me share) all the gossips, for giving feedback on my internet dates but also for supporting me in the more difficult times. And guess what? At my new job there is this bin-bag-banana issue as well, but luckily I haven't been placed on the naughty step yet...

Youri, Catalina, Camilo, Edgar, Luci and Cristina. Thanks for being such great company during our Romania trip after the wedding. For me this trip at the end of my PhD-life was a real 'battery charger' and looking back to it makes me smile. We should catch up soon!

Ivon (en Martyn), bedankt voor het altijd klaar staan en de onvoorwaardelijke steun in voor en tegenspoed. Jammer dat jullie er niet bij kunnen zijn op 9 september, succes met de bevalling van de $2^{\text {e }}$, en van je proefschrift! Alexandra (en Martien), fijn om altijd ervaringen te kunnen uitwisselen over het wel en wee van het promoveren, laten we snel weer bij praten!

Hans T., Packo, Miranda, Jan de frikandellenman, Marina, Fabian, Dirk, Mark, Carl, Rafael, Carsten, Douwe, Gerard, Mathieu, Shirley, Rouke, Vincent, Sina, Imma, Klaske, Petra, Dorinde, Nadia, Nadine, Ellen, Abdulaziz, Ruchir, Rupali, Giulia, Michiel, Michel, Niels-Henrik, Rik, Edwin, Maria B., Giuseppe, Hans R., Marjan, Ruud, Marta, Martin, Pascalle, Jacqueline, Karolina, Anja, Kelly, Pieter and all the other FPE and BPE staff and students, thanks for the great time I had during borrels, koffiepauzes, WE-days and Christmas dinners!

Mijn lieve ouders, en Linda, Bas en kleine Daan, bedankt voor alle steun, vertrouwen en aanmoediging, de heerlijk stabiele thuissituatie en het me laten realiseren dat niet alles om het proefschrift draait.

Het afronden van een proefschrift en het starten van een relatie is geen handige combinatie. Lieve Nena, ik ben zo blij dat wij die stap toch gezet hebben en dat we zijn waar we nu zijn! Bedankt voor je onvoorwaardelijke steun, liefde en doorzettingsvermogen. Wij hebben die vakantie naar de Azoren in september meer dan verdiend! -X- 


\section{Curriculum Vitae}




\section{About the author}

Tim de Mooij was born on January 13, 1987 in Deventer, The Netherlands. He went to Etty Hillesum Lyceum in Deventer where he obtained his VWO diploma in 2006, with a major in Natuur en Gezondheid. That same year he started to study Biotechnology at Wageningen University. He graduated from his BSc in 2009 on the topic of microalgae harvesting by means of flocculation at the department of Bioprocess Engineering. Hereafter he continued his MSc with as

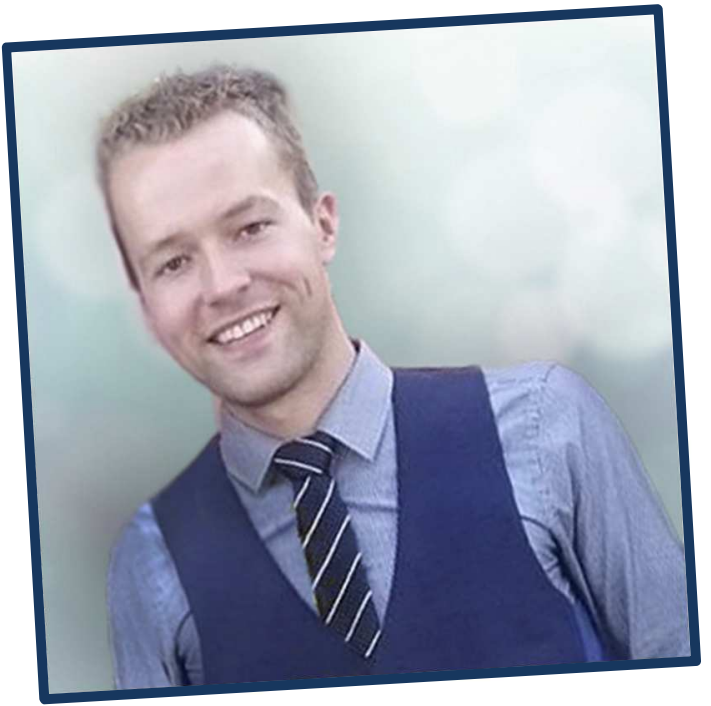
specialization 'Marine Biotechnology'. In 2011 Tim finished his MSc thesis about the accumulation of lipids in microalgae by applying different nutrient limitations. He followed an Internship at Ingrepro Renewables B.V. in Borculo, where he worked on immobilized growth of microalgae in alginate beads with the purpose of waste water effluent polishing using a novel type of photobioreactor.

In 2011, Tim obtained his MSc degree and started his PhD research at the Bioprocess Engineering department of the Wageningen University. He studied whether reduction of the photosynthetic antenna can increase biomass productivity of microalgae in mass culture and what the implications are for the cultivation strategy. The project was conducted within the research programme of BioSolar Cells. The results from these four years of research are described in this thesis.

Since April 2016, Tim works as a scientist / technical specialist at MSD Animal Health in Boxmeer. In this role he is involved in projects with technological challenges, he identifies and tests new technologies and implements them into existing production processes. 


\section{Overview of completed training activities}

\section{Discipline specific courses}

Advanced thermodynamics

2014

Bioreactor design and operation

2014

Numerical methods for chemical engineering

2014

Advanced course microbial physiology and fermentation technology

2015

\section{General courses}

Promotie in eigen regie (FOM)

VLAG PhD week, Baarlo, 25-28 October

Teaching and supervising thesis students

Scientific writing

Project and time management

Networking course

PhD competence assessment

Basic statistics

Career perspectives

\section{Conferences}

$1^{\text {st }}$ Young Algaeneers Symposium, Wageningen,

European congress of chemical engineering (ECCE), Den Haag

\section{Optionals}

Brainstorm/PhD-students days Bioprocess Engineering Group

\section{Teaching}


The work in this thesis was performed at the Laboratory of Bioprocess Engineering, Wageningen University, Wageningen, The Netherlands.

This work is part of the research programme of the Foundation for Fundamental Research on Matter (FOM) which is part of the Netherlands Organization for Scientific Research (NWO).

This project was conducted within the research programme of BioSolar Cells, cofinanced by the Dutch Ministry of Economic Affairs, Agriculture and Innovation.

This thesis was printed by Uitgeverij BOXPress || Proefschriftmaken.nl 